Physiologically based kinetic modeling based prediction of oral systemic bioavailability of flavonoids, their metabolites, and their biological effects 


\section{Thesis committee}

\section{Promotor}

Prof. Dr I.M.C.M. Rietjens

Professor of Toxicology

Wageningen University \& Research

\section{Co-promotor}

Dr A. Punt

Researcher DLO

RIKILT-Wageningen University \& Research

\section{Other members}

Prof. Dr J. Keijer, Wageningen University \& Research

Prof. Dr I.C.W. Arts, Maastricht University

Dr M.J. Zeilmaker, National Institute for Public Health and the Environment (RIVM),

Bilthoven

Dr W. Chanput - Kasetsart University, Chatuchak Bangkok, Thailand

This research was conducted under the auspices of the Graduate School VLAG (Advanced studies in Food Technology, Agrobiotechnology, Nutrition and Health Sciences) 


\title{
Physiologically based kinetic modeling based prediction of oral systemic bioavailability of flavonoids, their metabolites, and their biological effects
}

\author{
Rungnapa Boonpawa
}

\author{
Thesis \\ submitted in fulfilment of the requirements for the degree of doctor \\ at Wageningen University \\ by the authority of the Rector Magnificus \\ Prof. Dr A.P.J. Mol, \\ in the presence of the \\ Thesis Committee appointed by the Academic Board \\ to be defended in public \\ on Monday $23^{\text {rd }}$ of January 2017 \\ at 4 p.m. in the Aula.
}


Rungnapa Boonpawa

Physiologically based kinetic modelling based prediction of oral systemic bioavailability of flavonoids, their metabolites, and their biological effects

180 pages.

$\mathrm{PhD}$ thesis, Wageningen University \& Research, Wageningen, NL (2017)

With references, with summary in English

ISBN: 978-94-6343-037-1

DOI: http://dx.doi.org/10.18174/400192 


\section{TABLE OF CONTENTS}

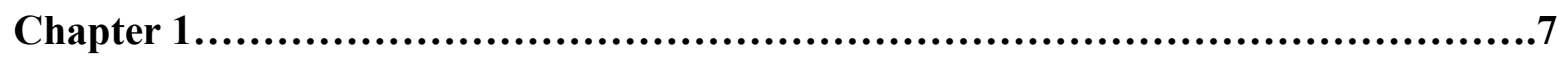

General introduction

Chapter 2................................................................................21

A physiologically based kinetic (PBK) model describing plasma

concentrations of quercetin and its metabolites in rats

Chapter 3

Use of physiologically based kinetic (PBK) modeling to study

interindividual human variation and species differences in plasma

concentrations of quercetin and its metabolites

Chapter 4.

In vitro-in silico based analysis of the dose-dependent in vivo

estrogenicity of the soy phytoestrogen genistein in humans

Chapter 5.

Physiologically based kinetic modeling of hesperidin metabolism and

its use to predict in vivo effective doses in humans

Chapter 6.

General discussion and future perspectives

Summary. .174

Acknowledgements..............................................................175

About the author....................................................................177

List of publications..............................................................178

Overview of completed training activities.........................................179 



\section{CHAPTER 1}

General introduction 


\subsection{Background information}

Based on epidemiological studies protective effects of fruits and vegetables against degenerative conditions including cancer [1], cardiovascular diseases [2], obesity and diabetes $[3,4]$, as well as neurodegenerative disorders [5, 6] have been suggested. Among many dietary phytochemicals, phenolic compounds especially flavonoids have perhaps attracted the most attention and are believed to be responsible, at least partly, for these protective health effects. This truly sparked the interest in the beneficial health effects of flavonoids and as a consequence numerous studies have been carried out in the past decades to determine the beneficial health effects of flavonoids [7].

Possible modes of action underlying the beneficial health effects of flavonoids, including their activity as ligands for various receptors, kinases, enzymes, and/or transport proteins, have been often studied using in vitro test system [7-9]. Although such in vitro results can be informative on the possible mechanisms involved in health effects of flavonoids, these results cannot be directly applied to humans. This is because in vitro studies have mostly been performed with flavonoid aglycones at high concentrations far beyond the plasma concentrations that can be achieved upon dietary or even supplementary intake in humans [8]. Pharmacokinetic studies have shown that flavonoids are extensively metabolized and thus mainly available in the systemic circulation in their conjugated forms [10]. The review article of Beekmann et al. [9] indicated that conjugation can affect biological activities as compared to the aglycone depending on the type and position of the conjugation. Altogether, the in vivo effects of flavonoids will ultimately depend on the systemic bioavailability of the flavonoid aglycones and their metabolites. As a result absorption, distribution, metabolism, and excretion (ADME) represent important factors determining the biological activity of flavonoids in vivo [11]. The aim of the present $\mathrm{PhD}$ project was to gain quantitative insight into the dose-dependent plasma concentrations of flavonoid aglycones and their metabolites using a physiologically based kinetic (PBK) modeling approach, and the flavonoids quercetin, genistein and hesperitin as model compounds.

\subsection{Flavonoids}

Flavonoids are a diverse group of polyphenolic compounds vastly distributed in the plant kingdom, with more than 6000 flavonoids identified so far [12]. They are secondary metabolites of plants and generally protect the plant against a variety of stress factors [13]. The basic chemical structure of flavonoids consists of 15 carbons, with two aromatic rings (A 
and B) connected by a three carbon bridge (ring C) as shown in Figure 1.1 [12]. Depending on the degree of oxidation of the heterocyclic ring $\mathrm{C}$, flavonoids can be classified into six major subclasses including flavonols, flavanones, flavan-3-ols, flavones, anthocyanidins, and isoflavones [14]. Flavonoids are commonly present in edible fruits and vegetables, but the type and amount of flavonoids present vary with the different dietary sources [12,14].

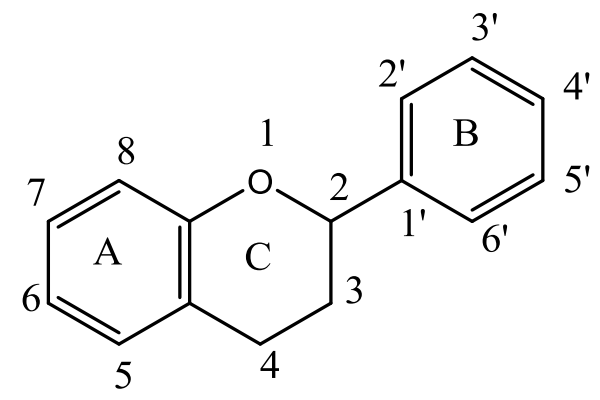

Figure 1.1 Basic chemical structure of flavonoids [12].

\subsubsection{Quercetin}

Quercetin (3,5,7,3',4'-pentahydroxyflavone) belongs to the flavonol subclass widely distributed in fruits and vegetables. It is one of the most prevalent flavonoids in human diets and also one of the most widely studied flavonoids [15]. The major dietary source of quercetin is onion followed by lettuce, red wine, and apple [16]. Dietary intake of quercetin is estimated to be $15-40 \mathrm{mg} /$ day in Western countries [17]. In some countries, quercetin is widely available as food supplement with recommended daily intakes of $250-1500 \mathrm{mg}$ quercetin/day [17]. Bioavailability of quercetin aglycone has been reported to be very low due to extensive metabolism, as no quercetin aglycone or only a trace amount of the aglycone has been detected in animal $[15,18,19]$ or in human plasma [20-22]. Quercetin naturally occurs as $\beta$-glycosides, with the type of sugar moiety varying depending on the dietary source. In onions, for example, quercetin is mainly present in the form of glucosides, whereas in apples it is mainly present as arabinoside, galactoside, glucoside, and rhamnoside [23]. The sugar moiety is key in determining the site of absorption [24]. Only glucosides are efficiently hydrolyzed prior to absorption in the small intestine by lactase phlorizin hydrolase (LPH), a $\beta$-glucosidase on the outside of the brush border membrane of the small intestine [24]. Other glycosides that are not substrates for LPH will be moved toward the colon where they are subjected to colonic metabolism leading to removal of the sugar moiety before quercetin is taken up in the colonic enterocytes [24]. The aglycone can be directly taken up in the small 


\section{Chapter 1}

intestine by passive diffusion [24]. The absorbed quercetin is metabolized mainly by uridine 5'-diphospho-glucuronosyl transferases (UGTs), sulfotransferases (SULTs), and catechol Omethyltransferases (COMTs) forming several mono-conjugates at the 3-, 7-, 3'-, and/or 4'position as depicted in Figure 1.2 [25, 26]. Further conjugation of mono-conjugates is likely to happen at the available $\mathrm{OH}$-positions and numerous different glucuronidated, sulfated, and/or methylated di- or tri-conjugates have been identified in plasma [18, 23, 27-30]. The major circulating metabolites of quercetin have been reported to be di- and tri-conjugates containing glucuronide, sulfate, and/or methyl moieties in rats $[18,27,28]$ and monoconjugates containing a glucuronide or sulfate moiety in humans [23, 29, 30], indicating species differences in the metabolism of quercetin. Quercetin conjugates are excreted from the systemic circulation via intestinal excretion (upon efflux transport back to the intestinal lumen), and biliary and urinary excretion $[19,31]$. Both in rats and humans, quercetin is also excreted as $\mathrm{CO}_{2}$ accounting for 30-60\% of intravenously administered quercetin [32, 33].

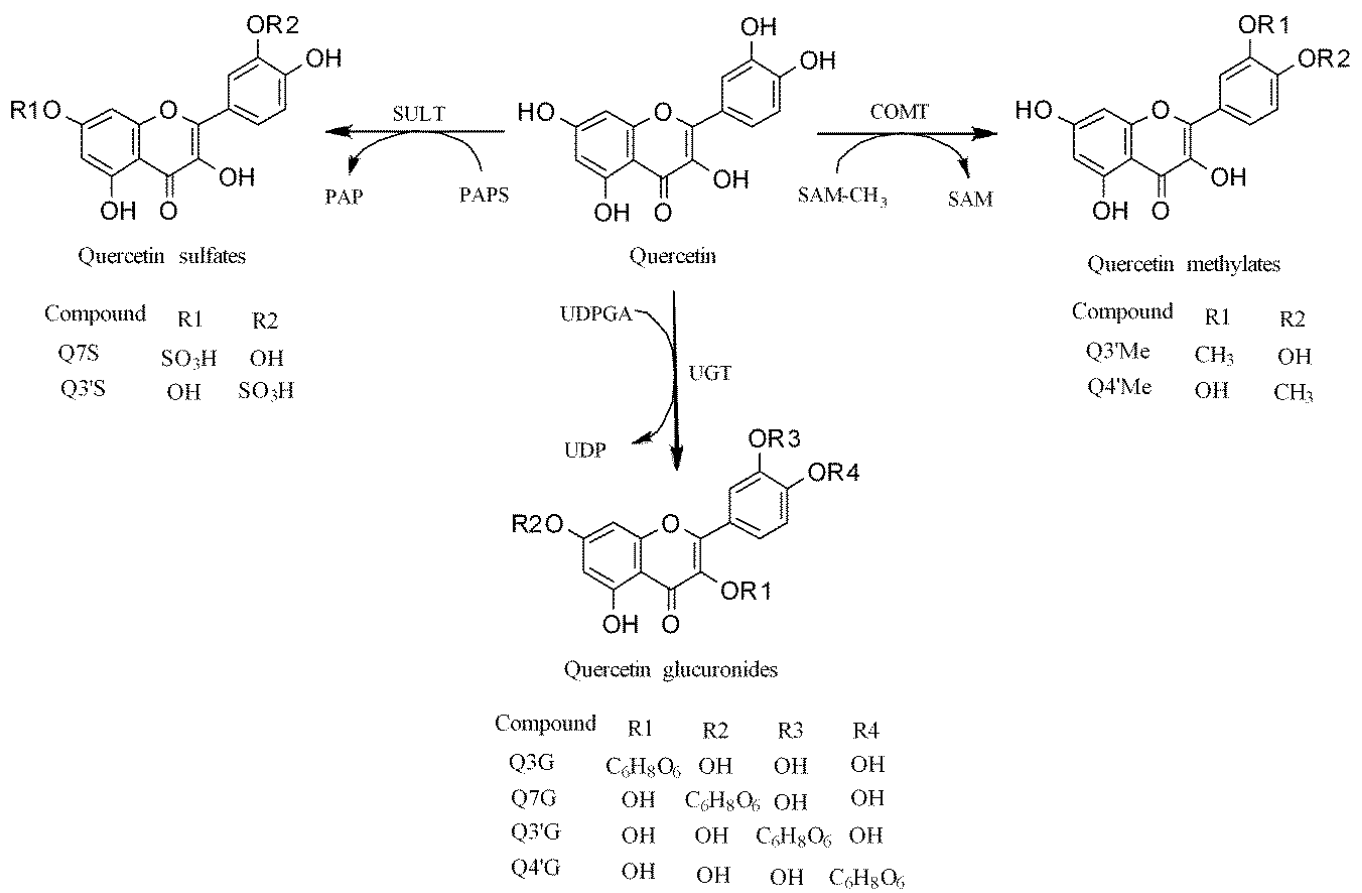

Figure 1.2 Metabolic conversion of quercetin to different mono-conjugates [25, 26].

\subsubsection{Genistein}

Genistein (4', 5, 7-trihydroxyisoflavone) belongs to the isoflavone subclass and is predominantly present in soy and soy-based products [34]. In soy, genistein mainly exists in a glycosylated form, genistin (genistein-7-O-glucoside). Dietary intake of genistein/isoflavones is estimated to be low in Western countries amounting to $<1 \mathrm{mg} /$ day [35], whereas in Asian countries genistein/isoflavones are regularly consumed at high oral doses amounting to 15-50 
$\mathrm{mg} /$ day [36]. Genistein is also available as food supplement either as pure genistein or in a mixture with other isoflavones at levels that would result in an estimated daily intake of 30$900 \mathrm{mg} /$ day [35]. Bioavailability of genistein aglycone has been recently reviewed and this overview showed low bioavailability in animals (6.8-23.4\% at 4-20 mg genistein/kg bw) and in humans (though for humans the absolute value is not available), mainly due to extensive metabolism of genistein in vivo [34]. Figure 1.3 depicts the proposed metabolic pathways of genistin in vivo [37-39]. Upon oral ingestion, the glycosylated genistin is hydrolyzed to the aglycone genistein by LPH in the small intestine [40] and/or by gut microflora in the colon when LPH is not sufficiently efficient to completely cleave the sugar moiety [41]. The absorbed genistein undergoes metabolism by UGTs and SULTs forming different monoconjugates at the 4'-and/or 7-position [42]. The mono-conjugates can be conjugated to a further extent at the available free $4^{\prime}$ or $7 \mathrm{OH}$-position resulting in formation of different diconjugates [37-39]. Gu et al. reported that $78.4 \%$ of the total genistein in human plasma consisted of glucuronidated conjugates, $20.4 \%$ being sulfated conjugates, and $1.2 \%$ being present as the aglycone [43]. In rat plasma genistein was reported to be present mainly as glucuronidated conjugates (50.4\%) and sulfated conjugates (45.9\%) with $3.6 \%$ being present as the aglycone [43]. Thus, there appear to be some species differences in metabolism of genistein between human and rat [43]. Genistein and its metabolites are distributed in tissues and plasma via the systemic circulation. Bolca et al. report that genistein aglycone was present at relatively low concentration in breast tissue (tissue $(12 \pm 2 \mathrm{pmol} / \mathrm{g})$ as compared to plasma $(67 \pm 19 \mathrm{nmol} / \mathrm{l})$ and the aglycone only accounted for $2 \%$ of total genistein (aglycone and metabolites) in women receiving $50.94 \mathrm{mg}$ genistein daily for 5 days [44]. Genistein is excreted mainly as conjugates via intestinal excretion (upon efflux back to the intestinal lumen), and biliary and urinary excretions [34]. The cumulative urinary excretion of genistein metabolites is reported to vary from $8-30 \%$ of the dose in humans [34]. The major excreted metabolites are reported to be glucuronides mainly genistein-7-O-glucuronide in both rats and in humans [34, 38, 39]. 


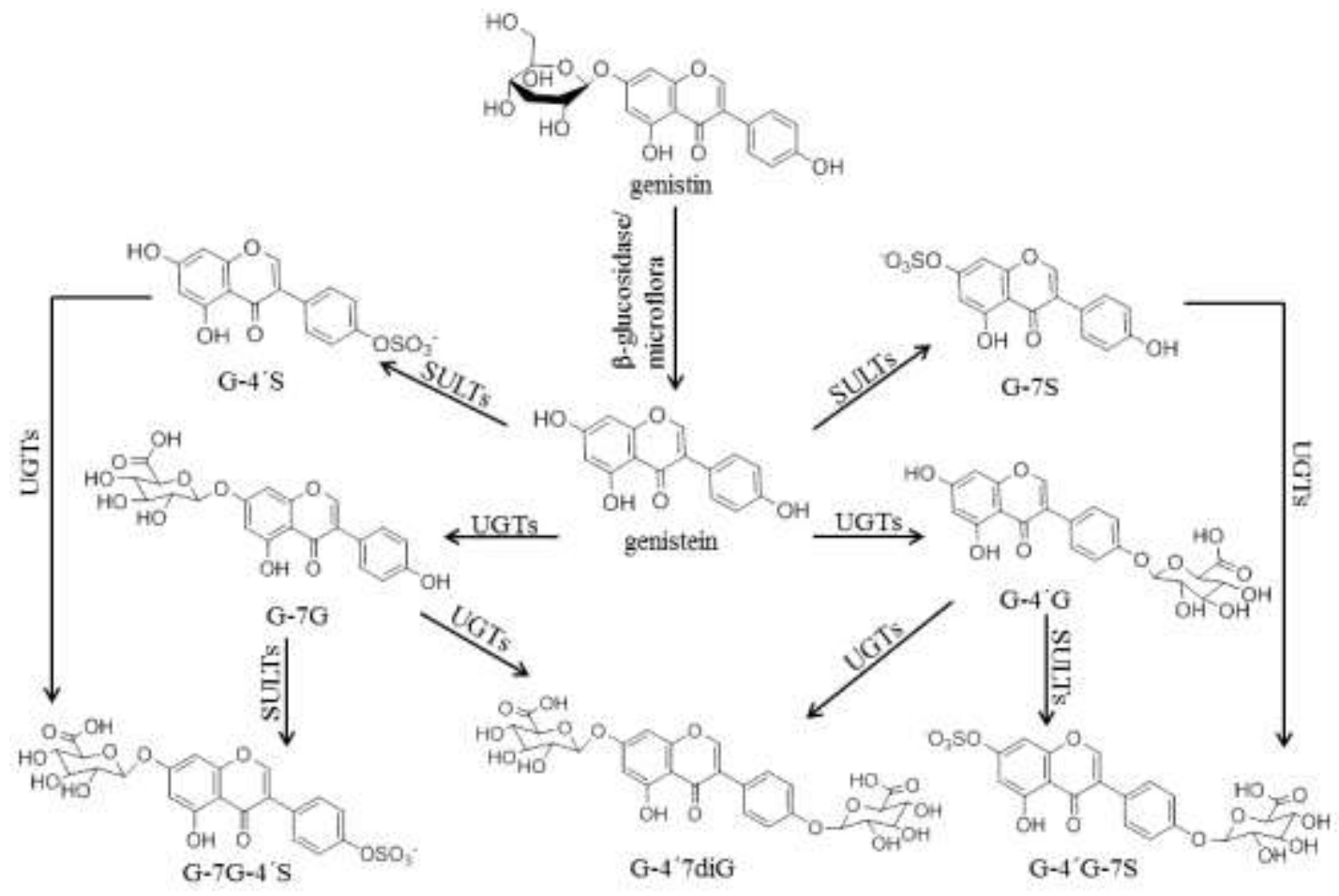

Figure 1.3 Proposed metabolic pathways of genistin in vivo [37-39].

\subsubsection{Hesperetin}

Hesperetin (3',5,7-trihydroxy-4'-methoxyflavanone) is a flavanone widely distributed in citrus fruits and naturally existing as hesperidin (hesperetin-7-O-rutinoside) [45]. Dietary intake of hesperetin is estimated to be 1.41-61.11 mg/day in Western countries [16]. When hesperetin is consumed as its glycoside, hesperidin, it is absorbed only in the distal part of the intestine after hydrolysis by colonic microflora [46]. The release of hesperetin aglycone is reported to be the rate-limiting step for the absorption [47]. In contrast, the hesperetin aglycone can be directly absorbed in the upper intestine by passive diffusion [46]. The absorbed hesperetin is metabolized by UGTs and SULTs at the 3'- and 7-position in the colon, small intestine, and liver forming different mono-conjugates as shown in Figure 1.4 [48-50]. Mono-conjugates are further metabolized by UGTs and SULTs at the available free $\mathrm{OH}$ positions forming different di-conjugates. Bioavailability of hesperetin aglycone is low in humans as hesperetin is mainly present as monoglucuronides in human plasma [46-49]. The total urinary excretion of conjugated hesperetin in human varied from 3.9-6.7\% of the dose after consumption of a diet containing hesperidin [47-49, 51]. 


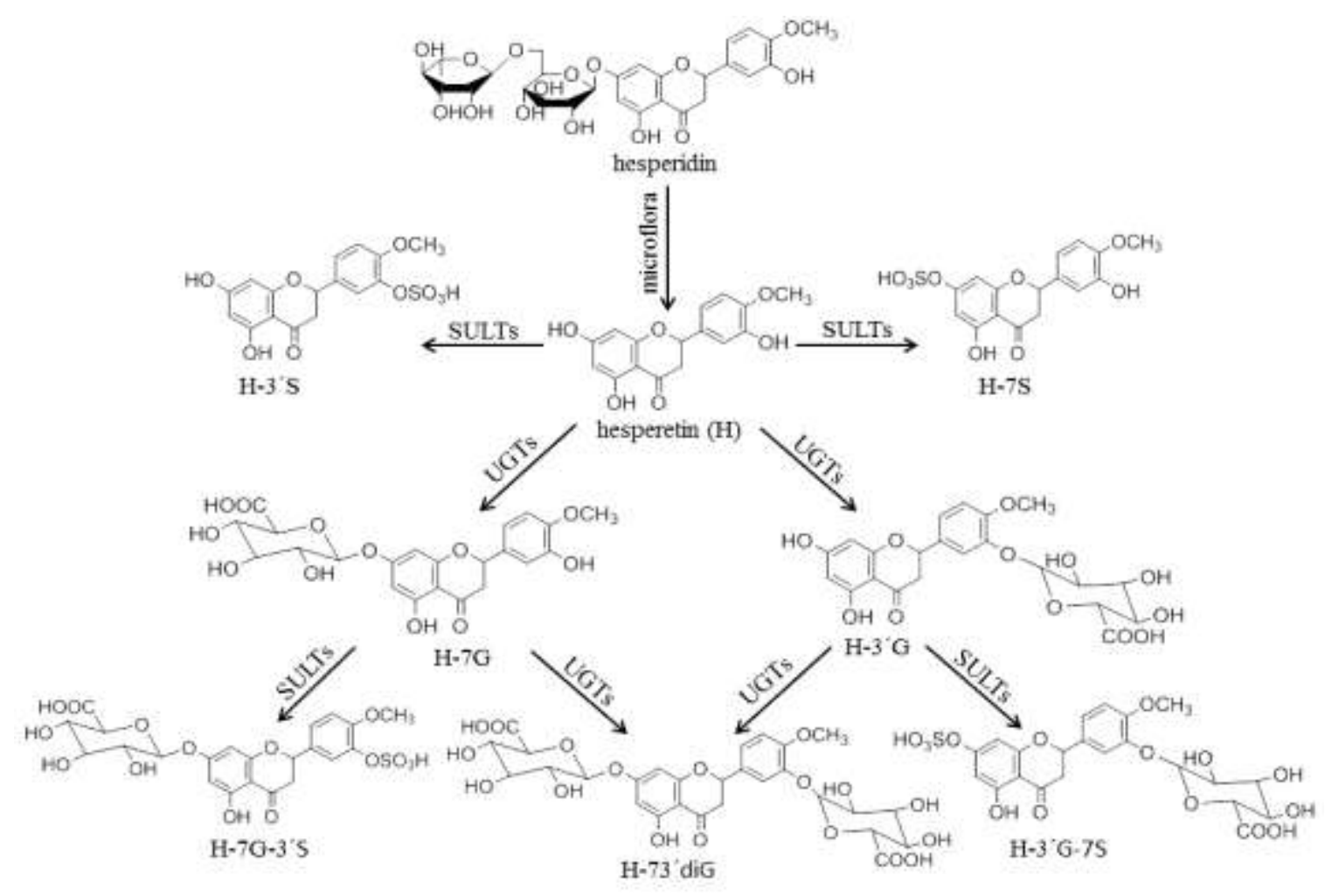

Figure 1.4 Proposed metabolic pathways of hesperidin in vivo [48-50].

\subsection{PBK models}

In vivo data reveal that flavonoids are hardly available as aglycone, but mainly present as conjugates [10]. These conjugates might contribute, at least partly, to the observed beneficial health effects of flavonoids in vivo. The possible effects of conjugation of flavonoids on the biological activity have been recently reviewed by Beekmanm et al. [9]. The results indicate that depending on the type and position of the conjugation as well as the endpoint studied, conjugates are less active or equally active as the aglycone, whereas in some cases the conjugates are more active than the aglycone or even showed an inverse activity [9]. Altogether, biological activity of flavonoids in vivo will ultimately depend on the systemic bioavailability of the flavonoid aglycones and their metabolites. Therefore, it is of importance to gain insight in the ADME of flavonoids, resulting in the better understanding of flavonoid in vivo effects.

PBK modeling describes the ADME of chemicals with a set of mathematical equations and allows simulation of plasma and tissue concentrations at low- and high-dose levels as well as evaluation of inter- and intraspecies differences [52-54]. Furthermore, the effect of kinetic interactions between different compounds, such as competition for 


\section{Chapter 1}

metabolizing enzymes, can be modeled based on in vitro derived kinetic constants for inhibition [55]. And by combining the PBK model with in vitro data, in a so-called reversedosimetry approach, in vivo dose-response curves can be predicted [56-58]. Overall, PBK modeling provides a powerful tool to explore the in vivo bioavailability as well as the ADME of a chemical.

A PBK model can be developed by integrating three type of parameters including 1) physiological and anatomical parameters (e.g. cardiac output, tissue volumes and tissue blood flows), 2) physio-chemical parameters (e.g. tissue:blood partition coefficients), and 3) kinetic constants for metabolic conversions [52]. The physiological and anatomical parameters can be obtained from literature [59]. Tissue:blood partition coefficients might be obtained experimentally in vitro using solid phase microextraction based approach [57]. Alternatively, tissue:blood partition coefficients can also be obtained using in silico methods based on octanol-water partition coefficient [60]. Kinetic parameters for ADME can be obtained using 1) experimental in vitro models using primary cell cultures, cells from cell lines in culture, subcellular fractions, tissue slices, or relevant recombinants enzymes of organs [61, 62], 2) available in silico methods [63-65], or 3) by fitting the model to available in vivo data [66]. By numerically integrating all mathematical equations for the ADME in computer software packages, the model can be simulated. Commonly used software is for example Berkeley Madonna (Macey and Oster, UC Berkeley, CA, USA), acsIXtreme (AEgis Technologies Group), and MATLAB (The MathWorks) [52]. Once the model is developed, it is of importance to evaluate the performance of the newly developed model against in vivo data, since this supports the validity of the model and thus its predictions.

\subsection{Objectives and outline of the thesis}

In vivo effects of flavonoids will ultimately depend on the availability of the flavonoid aglycones and their metabolites in the systemic circulation. The type and position of the conjugation of the circulating metabolites is also important to better understand in vivo effects of flavonoids [9]. The aim of the present $\mathrm{PhD}$ project was to gain quantitative insight in dosedependent plasma concentrations of flavonoid aglycones and their metabolites using a PBK modeling approach. The developed PBK models can be used to guide future experimental in vitro and in vivo studies as well as to facilitate the translation of in vitro results to the in vivo situation. Furthermore, the developed models have a great potential to be used as a platform to build PBK models for other flavonoids as we still lack in vivo kinetic data for many 
flavonoids. The present $\mathrm{PhD}$ project was focused on the flavonoids quercetin, genistein and hesperetin as model compounds.

In Chapter 1 of this thesis, an introduction to the fate of flavonoids in the body (ADME), focusing on quercetin, gensitein, and hesperetin, a short introduction to PBK modeling, and the aim and the outline of the present thesis are given. In Chapter 2, a PBK model for quercetin in rats was developed enabling prediction of plasma concentrations of quercetin aglycone and the tentative identification of the major circulating metabolites at different oral doses. The PBK model was built by integrating kinetic constants for metabolic conversions obtained from in vitro incubations with relevant rat tissue fractions and by fitting kinetic parameters to literature available data in rats. The PBK model developed in rats was used as a platform to build a PBK model for quercetin in humans as described in Chapter 3, facilitating interspecies extrapolation. In the human population, the activity of enzymes involved in the metabolism of quercetin can vary due to genotype- and lifestyle-based factors, which could influence the bioavailability of quercetin aglycone and its metabolites in the systemic circulation. Therefore, in Chapter 3 also individual PBK models were developed by incorporating kinetic constants for metabolic conversions obtained using individual human samples. For many flavonoids, unlike quercetin, in vivo kinetic data are still lacking, and therefore the developed quercetin models can form a basis for the development of similar models for other relevant dietary flavonoids such as genistein (soy-based isoflavone) and hesperetin (citrus-based flavanone). In Chapter 4, a PBK model for genistein in humans was developed based on the methods that were developed for simulating quercetin kinetics. Due to the weak estrogenicity of genistein, there is an increasing use of genistein for example in hormone replacement therapy in postmenopausal women. To assess whether at the current intake levels estrogenic activity of genistein can actually be achieved in humans, a reversedosimetry approach was applied combining in vitro genistein estrogenicity data with the developed PBK model for genistein to convert in vitro effect concentrations to in vivo effective dose levels, enabling prediction of in vivo estrogenic dose-response curves in humans. In Chapter 5, the PBK models for quercetin were further used as a platform to build a PBK model for hesperetin in humans to predict dose-dependent plasma concentrations of hesperetin aglycone and its metabolites. Also this PBK model was used to convert reported effect concentrations from in vitro bioassays to corresponding in vivo dose levels, in order to provide insight in whether the observed in vitro effects may be relevant under realistic dietary or supplementary intake. Finally, Chapter 6 presents main finding obtained in this thesis and provides a discussion on how these results can contribute to future research on flavonoids. 


\section{Chapter 1}

\section{References}

1. Neuhouser, M. L., Review: dietary flavonoids and cancer risk: evidence from human population studies. Nutrition and Cancer, 2004. 50(1): p. 1-7.

2. Perez-Vizcaino, F. and J. Duarte, Flavonols and cardiovascular disease. Molecular Aspects of Medicine, 2010. 31(6): p. 478-494.

3. Hughes, L. A., et al., Higher dietary flavone, flavonol, and catechin intakes are associated with less of an increase in BMI over time in women: a longitudinal analysis from the Netherlands Cohort Study. The American Journal of Clinical Nutrition, 2008. 88(5): p. 13411352.

4. Liu, Y.-J., et al., Dietary flavonoids intake and risk of type 2 diabetes: A meta-analysis of prospective cohort studies. Clinical Nutrition, 2014. 33(1): p. 59-63.

5. Letenneur, L., et al., Flavonoid intake and cognitive decline over a 10-year period. American Journal of Epidemiology, 2007. 165(12): p. 1364-1371.

6. Morris, M. C., et al., Associations of vegetable and fruit consumption with age-related cognitive change. Neurology, 2006. 67(8): p. 1370-1376.

7. Havsteen, B. H., The biochemistry and medical significance of the flavonoids. Pharmacology \& Therapeutics, 2002. 96(2-3): p. 67-202.

8. Williamson, G., et al., In vitro biological properties of flavonoid conjugates found in vivo. Free Radical Research, 2005. 39(5): p. 457-469.

9. Beekmann, K., et al., A state-of-the-art overview of the effect of metabolic conjugation on the biological activity of flavonoids. Food \& Function, 2012. 3(10): p. 1008-1018.

10. Manach, C., et al., Bioavailability and bioefficacy of polyphenols in humans. I. Review of 97 bioavailability studies. The American Journal of Clinical Nutrition, 2005. 81(1): p. 230S242S.

11. Kay, C. D., The future of flavonoid research. British Journal of Nutrition, 2010. 104(SupplementS3): p. S91-S95.

12. Erlund, I., Review of the flavonoids quercetin, hesperetin, and naringenin. Dietary sources, bioactivities, bioavailability, and epidemiology. Nutrition Research, 2004. 24(10): p. 851-874.

13. Manach, C., et al., Polyphenols: food sources and bioavailability. The American Journal of Clinical Nutrition, 2004. 79(5): p. 727-747.

14. Ross, J. A. and C. M. Kasum, Dietary flavonoids: bioavailability, metabolic effects, and safety. Annual Review of Nutrition, 2002. 22(1): p. 19-34.

15. de Boer, V. C. J., et al., Tissue distribution of quercetin in rats and pigs. The Journal of Nutrition, 2005. 135(7): p. 1718-1725.

16. Zamora-Ros, R., et al., Estimation of dietary sources and flavonoid intake in a spanish adult population (EPIC-Spain). Journal of the American Dietetic Association, 2010. 110(3): p. 390398.

17. Larson, A., J. D. Symons, and T. Jalili, Quercetin: A treatment for hypertension?-A review of efficacy and mechanisms. Pharmaceuticals, 2010. 3(1): p. 237-250.

18. Graf, B. A., et al., Disposition and metabolism of $\left[2-{ }^{14} \mathrm{C}\right]$ quercetin-4'-glucoside in rats. Drug Metabolism and Disposition, 2005. 33(7): p. 1036-1043.

19. Chen, X., et al., Pharmacokinetics and modeling of quercetin and metabolites. Pharmaceutical Research, 2005. 22(6): p. 892-901.

20. Olthof, M. R., et al., Bioavailabilities of quercetin-3-glucoside and quercetin-4'-glucoside do not differ in humans. The Journal of Nutrition, 2000. 130(5): p. 1200-1203.

21. Gugler, R., M. Leschik, and H. J. Dengler, Disposition of quercetin in man after single oral and intravenous doses. European Journal of Clinical Pharmacology, 1975. 9(2): p. 229-234. 
22. Graefe, E. U., et al., Pharmacokinetics and bioavailability of quercetin glycosides in humans. The Journal of Clinical Pharmacology, 2001. 41(5): p. 492-499.

23. Lee, J., et al., UHPLC-(ESI)QTOF MS/MS profiling of quercetin metabolites in human plasma postconsumption of applesauce enriched with apple peel and onion. Journal of Agricultural and Food Chemistry, 2012. 60(34): p. 8510-8520.

24. Hollman, P. C. H., Absorption, bioavailability, and metabolism of flavonoids. Pharmaceutical Biology, 2004. 42(sup1): p. 74-83.

25. Boersma, M. G., et al., Regioselectivity of phase II metabolism of luteolin and quercetin by UDP-glucuronosyl transferases. Chemical Research in Toxicology, 2002. 15(5): p. 662-670.

26. van der Woude, H., et al., Identification of 14 quercetin phase II mono- and mixed conjugates and their formation by rat and human phase II in vitro model systems. Chemical Research in Toxicology, 2004. 17(11): p. 1520-1530.

27. Mullen, W., et al., Determination of flavonol metabolites in plasma and tissues of rats by HPLC-radiocounting and tandem mass spectrometry following oral ingestion of [214C]quercetin-4'-glucoside. Journal of Agricultural and Food Chemistry, 2002. 50(23): p. 6902-6909.

28. Graf, B. A., et al., Rat gastrointestinal tissues metabolize quercetin. The Journal of Nutrition, 2006. 136(1): p. 39-44.

29. Mullen, W., C. A. Edwards, and A. Crozier, Absorption, excretion and metabolite profiling of methyl-, glucuronyl-, glucosyl- and sulpho-conjugates of quercetin in human plasma and urine after ingestion of onions. British Journal of Nutrition, 2006. 96: p. 107-116.

30. Day, A. J., et al., Human metabolism of dietary flavonoids: Identification of plasma metabolites of quercetin. Free Radical Research, 2001. 35(6): p. 941-952.

31. Crespy, V., et al., Part of quercetin absorbed in the small intestine is conjugated and further secreted in the intestinal lumen. American Journal of Physiology - Gastrointestinal and Liver Physiology, 1999. 277(1): p. G120-G126.

32. Ueno, I., N. Nakano, and I. Hirono, Metabolic fate of [14C] quercetin in the ACl rat. Japanese Journal of Experimental Medicine, 1983. 53(1): p. 41-50.

33. Walle, T., U. K. Walle, and P. V. Halushka, Carbon dioxide is the major metabolite of quercetin in humans. The Journal of Nutrition, 2001. 131(10): p. 2648-2652.

34. Yang, Z., et al., Bioavailability and pharmacokinetics of genistein: mechanistic studies on its ADME. Anti-cancer agents in medicinal chemistry, 2012. 12(10): p. 1264-1280.

35. EFSA, Risk assessment for peri- and post-menopausal women taking food supplements containing isolated isoflavones. EFSA Journal 2015, 2015. 13(10): p. 4246 [page 342].

36. Rietjens, I. M. C. M., et al., Mechanisms underlying the dualistic mode of action of major soy isoflavones in relation to cell proliferation and cancer risks. Molecular Nutrition \& Food Research, 2013. 57(1): p. 100-113.

37. Hosoda, K., et al., Identification and quantification of daidzein-7-glucuronide-4'-sulfate, genistein-7-glucuronide-4'-sulfate and genistein-4',7-diglucuronide as major metabolites in human plasma after administration of kinako. Analytical and Bioanalytical Chemistry, 2010. 397(4): p. 1563-1572.

38. Hosoda, K., T. Furuta, and K. Ishii, Metabolism and disposition of isoflavone conjugated metabolites in humans after ingestion of kinako. Drug Metabolism and Disposition, 2011. 39(9): p. 1762-1767.

39. Yuan, B., et al., Absorption and plasma disposition of genistin differ from those of genistein in healthy women. Journal of Agricultural and Food Chemistry, 2012. 60(6): p. 1428-1436. 
40. Walsh, K. R., et al., Isoflavonoid glucosides are deconjugated and absorbed in the small intestine of human subjects with ileostomies. The American Journal of Clinical Nutrition, 2007. 85(4): p. 1050-1056.

41. Bokkenheuser, V. D., C. H. Shackleton, and J. Winter, Hydrolysis of dietary flavonoid glycosides by strains of intestinal Bacteroides from humans. Biochemical Journal, 1987. 248(3): p. 953-956.

42. Doerge, D. R., et al., Analysis of soy isoflavone conjugation in vitro and in human blood using liquid chromatography-mass spectrometry. Drug Metabolism and Disposition, 2000. 28(3): p. 298-307.

43. Gu, L., et al., Metabolic phenotype of isoflavones differ among female rats, pigs, monkeys, and women. The Journal of Nutrition, 2006. 136(5): p. 1215-1221.

44. Bolca, S., et al., Disposition of soy isoflavones in normal human breast tissue. The American Journal of Clinical Nutrition, 2010. 91(4): p. 976-984.

45. Pereira-Caro, G., et al., Orange juice (poly)phenols are highly bioavailable in humans. The American Journal of Clinical Nutrition, 2014. 100(5): p. 1378-1384.

46. Takumi, H., et al., Bioavailability of orally administered water-dispersible hesperetin and its effect on peripheral vasodilatation in human subjects: implication of endothelial functions of plasma conjugated metabolites. Food \& Function, 2012. 3(4): p. 389-398.

47. Manach, C., et al., Bioavailability in humans of the flavanones hesperidin and narirutin after the ingestion of two doses of orange juice. European Journal of Clinical Nutrition, 2003. 57(2): p. 235-242.

48. Brett, G. M., et al., Absorption, metabolism and excretion of flavanones from single portions of orange fruit and juice and effects of anthropometric variables and contraceptive pill use on flavanone excretion. British Journal of Nutrition, 2009. 101(05): p. 664-675.

49. Mullen, W., et al., Bioavailability and metabolism of orange juice flavanones in humans: impact of a full-fat yogurt. Journal of Agricultural and Food Chemistry, 2008. 56(23): p. 11157-11164.

50. Perche, O., et al., Orange juice and its major polyphenol hesperidin consumption do not induce immunomodulation in healthy well-nourished humans. Clinical Nutrition, 2013. 33(1): p. $130-135$.

51. Erlund, I., et al., Plasma kinetics and urinary excretion of the flavanones naringenin and hesperetin in humans after ingestion of orange juice and grapefruit juice. The Journal of Nutrition, 2001. 131(2): p. 235-241.

52. Rietjens, I. M. C. M., J. Louisse, and A. Punt, Tutorial on physiologically based kinetic modeling in molecular nutrition and food research. Molecular Nutrition \& Food Research, 2011. 55(6): p. 941-956.

53. Punt, A., et al., A physiologically based biokinetic (PBBK) model for estragole bioactivation and detoxification in rat. Toxicology and Applied Pharmacology, 2008. 231(2): p. 248-259.

54. Punt, A., et al., Use of physiologically based biokinetic (PBBK) modeling to study estragole bioactivation and detoxification in humans as compared with male rats. Toxicological Sciences, 2009. 110(2): p. 255-269.

55. Alhusainy, W., et al., Identification of nevadensin as an important herb-based constituent inhibiting estragole bioactivation and physiology-based biokinetic modeling of its possible in vivo effect. Toxicology and Applied Pharmacology, 2010. 245(2): p. 179-190.

56. Louisse, J., et al., The use of in vitro toxicity data and physiologically based kinetic modeling to predict dose-response curves for in vivo developmental toxicity of glycol ethers in rat and man. Toxicological Sciences, 2010. 118(2): p. 470-484. 
57. Louisse, J., et al., Prediction of in vivo developmental toxicity of all-trans-retinoic acid based on in vitro toxicity data and in silico physiologically based kinetic modeling. Archives of Toxicology, 2015. 89(7): p. 1135-1148.

58. Strikwold, M., et al., Combining in vitro embryotoxicity data with physiologically based kinetic (PBK) modelling to define in vivo dose-response curves for developmental toxicity of phenol in rat and human. Archives of Toxicology, 2013. 87(9): p. 1709-1723.

59. Brown, R. P., et al., Physiological parameter values for physiologically based pharmacokinetic models. Toxicology and Industrial Health, 1997. 13(4): p. 407-484.

60. DeJongh, J., H. J. M. Verhaar, and J. L. M. Hermens, A quantitative property-property relationship (QPPR) approach to estimate in vitro tissue-blood partition coefficients of organic chemicals in rats and humans. Archives of Toxicology, 1997. 72(1): p. 17-25.

61. Lipscomb, J. C. and T. S. Poet, In vitro measurements of metabolism for application in pharmacokinetic modeling. Pharmacology \& Therapeutics, 2008. 118(1): p. 82-103.

62. Steensma, A., H. P. J. M. Noteborn, and H. A. Kuiper, Comparison of Caco-2, IEC-18 and HCEC cell lines as a model for intestinal absorption of genistein, daidzein and their glycosides. Environmental Toxicology and Pharmacology, 2004. 16(3): p. 131-139.

63. Yang, X., et al., Prediction of biliary excretion in rats and humans using molecular weight and quantitative structure-pharmacokinetic relationships. AAPS J., 2009. 11(3): p. 511-525.

64. Manga, N. n., et al., A hierarchical QSAR model for urinary excretion of drugs in humans as a predictive tool for biotransformation. QSAR \& Combinatorial Science, 2003. 22(2): p. 263273.

65. Levitt, D. G., Quantitation of small intestinal permeability during normal human drug absorption. BMC Pharmacology \& Toxicology, 2013. 14: p. 34-34.

66. Reddy, M. B., et al., Physiological modeling of inhalation kinetics of octamethylcyclotetrasiloxane in humans during rest and exercise. Toxicological Sciences, 2003. 72(1): p. 3-18. 
Chapter 1 


\section{CHAPTER 2}

A physiologically based kinetic (PBK) model describing plasma concentrations of quercetin and its metabolites in rats

Rungnapa Boonpawa,

Albertus Spenkelink, Ivonne M. C. M. Rietjens, Ans Punt

Published in:

Biochemical Pharmacology 2014; 89(2): 287-299. 


\begin{abstract}
Biological activities of flavonoids in vivo are ultimately dependent on the systemic bioavailability of the aglycones as well as their metabolites. In the present study, a physiologically based kinetic (PBK) model was developed to predict plasma concentrations of the flavonoid quercetin and its metabolites and to tentatively identify the regiospecificity of the major circulating metabolites. The model was developed based on in vitro metabolic parameters and by fitting kinetic parameters to literature available in vivo data. Both exposure to quercetin aglycone and to quercetin-4'-O-glucoside, for which in vivo data were available, were simulated. The predicted plasma concentrations of different metabolites adequately matched literature reported plasma concentrations of these metabolites in rats exposed to 4'O-glucoside. The bioavailability of aglycone was predicted to be very low ranging from $0.004 \%$ to $0.1 \%$ at different oral doses of quercetin or quercetin-4'-O-glucoside.
\end{abstract} Glucuronidation was a crucial pathway that limited the bioavailability of the aglycone, with 95 to $99 \%$ of the dose being converted to monoglucuronides within $1.5 \mathrm{~h}$ to $2.5 \mathrm{~h}$ at different dose levels ranging from 0.1 to $50 \mathrm{mg} / \mathrm{kg}$ bw quercetin or quercetin-4'-O-glucoside. The fast metabolic conversion to monoglucuronides allowed these metabolites to further conjugate to di- and tri-conjugates. The regiospecificity of major circulating metabolites was observed to be dose-dependent. As we still lack in vivo kinetic data for many flavonoids, the developed model has a great potential to be used as a platform to build PBK models for other flavonoids as well as to predict the kinetics of flavonoids in humans. 


\subsection{Introduction}

Flavonoids are important bioactive compounds widely distributed in plant based foods and often suggested to be of interest for their biological effects against several chronic and degenerative diseases, including cancer [1], cardiovascular diseases [2, 3], obesity and diabetes $[4,5]$, as well as neurodegenerative disorders [6, 7]. To elucidate the modes of action underlying the beneficial health effects, flavonoids are widely studied in vitro for their antioxidant activity as well as their activity as ligands for various receptors, kinases, enzymes, and/or transport proteins [8-11]. However, a key limitation in interpretation of these results is that in vitro data are often performed with the aglycone as the test compound [12]. In vivo effects will ultimately depend on the systemic bioavailability of the aglycone as well as its metabolites, though little is known about the contribution of circulating metabolites to the health effects or the precise nature of circulating metabolites. For a better interpretation of in vitro results and to better guide the experimental design of in vitro experiments with flavonoids, a clearer insight is needed on how flavonoids are absorbed, distributed, metabolized and excreted, especially to obtain an indication of the physiologically relevant concentrations of circulating metabolites in plasma and the precise nature of these metabolites.

The objective of the present study was to define a physiologically based kinetic (PBK) model that can predict plasma concentrations of the flavonoid quercetin and its metabolites and to tentatively identify the regiospecificity of the major circulating metabolites. Among many flavonoids, the flavonol quercetin is relatively abundant in our diet and the most frequently studied [13]. In Western countries, the total dietary intake of quercetin is estimated to be $15-40 \mathrm{mg}$ corresponding to $0.2-0.6 \mathrm{mg} / \mathrm{kg}$ body weight (bw) for a $70 \mathrm{~kg}$ person [3]. In some countries, quercetin is also available as a dietary supplement with daily doses of 250$1500 \mathrm{mg}$ quercetin corresponding to $3.6-21 \mathrm{mg}$ quercetin/ $\mathrm{kg}$ bw for a $70 \mathrm{~kg}$ person [3]. Naturally, quercetin occurs almost exclusively bound to one or more sugar molecules, mainly as $\beta$-glycosides in a wide variety of fruits and vegetables especially onions [3]. Quercetin glucosides such as 3,4'-O-diglucoside, 4'-O-glucoside, and 3-O-glucoside are the most abundant in onions [14]. Most industrial and domestic food processing procedures are not able to cleave the glycosidic linkage, except for fermentation [15] which implies that exposure via the diet is mostly to quercetin glucosides.

The glucoside forms of the quercetin are generally too polar to cross cellular membranes by diffusion, and this hampers their cellular uptake and bioavailability [16, 17]. 
Chapter 2

However, quercetin glucosides can be taken up in the intestine after being hydrolyzed into quercetin aglycone by luminal lactase phlorizin hydrolase or by intestinal microflora $[15,18]$. Upon absorption quercetin is metabolized by UDP-glucuronosyl transferases, sulfotransferases, and/or catechol O-methyltransferases in the small intestine and in the liver, forming different glucuronidated, sulfated and O-methylated quercetin mono-conjugates, respectively (Figure 2.1) [13, 19, 20]. Numerous different methylated, glucuronidated, and/or sulfated quercetin, di- and tri-conjugates have been identified in plasma in vivo as well, suggesting further metabolism of mono-conjugated metabolites in rats as well as in humans [21-25].

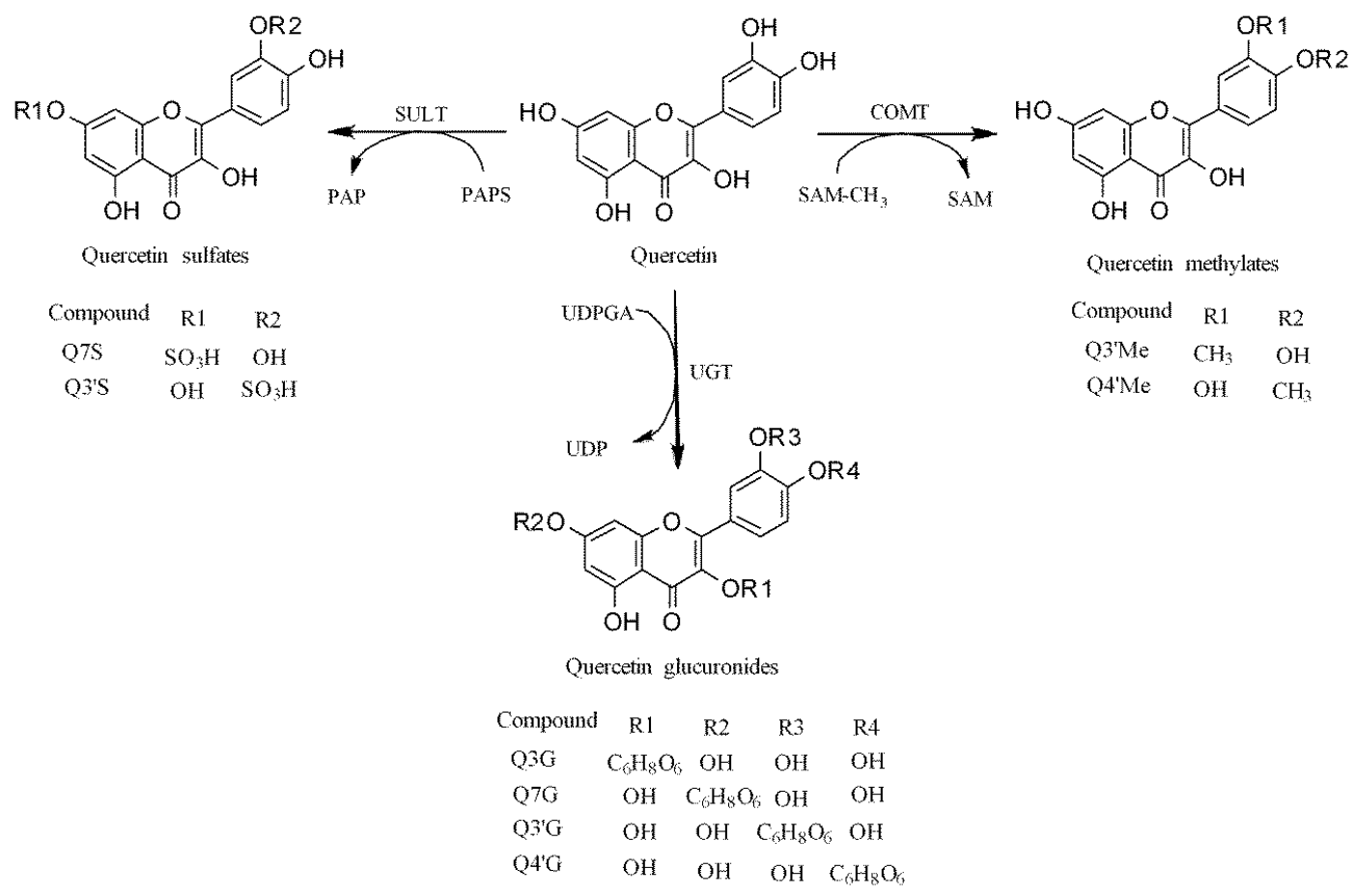

Figure 2.1 Conversion of quercetin to different mono-conjugates [19, 20].

To provide a better indication of the in vivo bioavailability of quercetin and the relative importance and structure of different circulating metabolites in plasma, we have developed a PBK model for quercetin in rats. To this end the kinetics for the formation of different quercetin metabolites where studied in vitro. Both a direct exposure to quercetin aglycone and to quercetin as its 4'-O-glucoside were simulated and evaluated by comparing the predicted total plasma concentrations of different metabolites obtained against available literature data obtained with rats exposed to quercetin-4'-O-glucoside [22]. The experiments and model of the present paper can set an example of how in vitro data can be integrated to predict in vivo plasma concentrations of flavonoids and their conjugated metabolites. 


\subsection{Material and methods}

\subsubsection{Materials}

Pooled male small intestine and liver S9 fractions from Sprague-Dawley rats were purchased from Xenotech (Lenexa, USA). Quercetin, L-ascorbic acid, uridine 5'diphosphoglucuronic acid (UDPGA, purity 98\%), S-(5'-adenosyl)-L-methionine (SAM, purity 80\%), 3'-phosphoadenosine 5'-phosphosulfate (PAPS, purity 65\%), tris (hydroxymethyl)aminomethane, alamethicin (from Trichoderma viride), and $\beta$-glucuronidase/sulfatase (from Helix pomatia) were purchased from Sigma-Aldrich (Steinheim, Germany). Potassium dihydrogen phosphate, dipotassium hydrogen phosphate trihydrate, acetic acid (glacial), hydrochloric acid $\left(\mathrm{HCl}\right.$, fuming 37\%), ammonium acetate, magnesium chloride $\left(\mathrm{MgCl}_{2}\right)$, sodium acetate trihydrate and trifluoroacetic acid (TFA, for spectroscopy) were purchased from VWR International (Darmstadt, Germany). Dimethyl sulfoxide (DMSO) was purchased from Acros Organic (New Jersey, USA). Acetonitrile (ULC/MS) was purchased from Biosolve (Valkenswaard, The Netherlands). Quercetin-3-O-glucuronide, isorhamnetin (3'-Omethylquercetin), and tamarixetin (4'-O-methylquercetin) were purchased from Extrasynthese (Genay Cedex, France).

\subsubsection{Glucuronidation of quercetin}

The kinetic constants for glucuronidation of quercetin were determined by performing incubations with pooled S9 fractions from male rat small intestine and liver (SpragueDawley). The incubation mixtures had a final volume of $100 \mu$, containing (final concentration) $0.1 \mathrm{M}$ Tris- $\mathrm{HCl}, \mathrm{pH} 7.4,10 \mathrm{mM} \mathrm{MgCl}_{2}, 1 \mathrm{mM}$ ascorbic acid to stabilize quercetin [20], $0.02 \mathrm{mg} / \mathrm{ml} \mathrm{S9}$ protein, and quercetin added from a 200 times concentrated stock solution in DMSO. The concentration of DMSO was kept at $0.5 \%$, which has previously shown to not have any effect on the enzyme activities [26]. Quercetin was stable for $2 \mathrm{~h}$ upon the addition of ascorbic acid at $37^{\circ} \mathrm{C}(90-100 \%$ of the dose remains after $2 \mathrm{~h}$ ). The incubation mixtures were pre-treated on ice with $0.025 \mathrm{mg} / \mathrm{ml}$ alamethicin added from a 200 times concentrated stock solution in methanol for $15 \mathrm{~min}$ to overcome enzyme latency and obtain maximal glucuronidation activity [27]. The reactions were initiated by adding the cofactor UDPGA ( $4 \mathrm{mM}$, final concentration) after preincubating at $37^{\circ} \mathrm{C}$ for $1 \mathrm{~min}$. The kinetic constants for glucuronidation of quercetin were determined at substrate concentrations 


\section{Chapter 2}

that ranged from 0.2 to $20 \mu \mathrm{M}$ quercetin. The reactions were terminated after 5 min by adding $25 \mu 1$ ice-cold acetonitrile:acetic acid (94:6) (v/v) to $100 \mu 1$ incubation mixture. Under these conditions, the formation of quercetin monoglucuronide conjugates was linear in time and with protein concentration (data not shown). Three replicates of each experiment were performed. Blank incubations were carried out without the cofactor. Identification of different monoglucuronides was done following UPLC analysis by comparing the UV-spectra and the order of elution of each metabolite with the data reported in the study of Boersma et al.[19]. The nature of these metabolites was indirectly confirmed by treating samples with $\beta$ glucuronidase. For this reaction, $50 \mu 1$ of the non-terminated mixtures where added to $50 \mu 1$ of $0.1 \mathrm{M}$ sodium acetate, $\mathrm{pH} 5$ containing $1 \mathrm{mM}$ ascorbic acid and $\beta$-glucuronidase 0.8 units $/ \mathrm{ml}$. Incubation was carried out for $1 \mathrm{~h}$ at $37^{\circ} \mathrm{C}$ and terminated by addition of $25 \mu \mathrm{l}$ ice-cold acetonitrile:acetic acid (94:6) (v/v). Samples were immediately analyzed by UPLC (Ultra Performance Liquid Chromatography) as described in section 2.2.5.

\subsubsection{Sulfation of quercetin}

The kinetic constants for sulfation of quercetin were determined by performing incubations with pooled S9 fractions from male rat small intestine and liver (SpragueDawley). The incubation mixtures had a final volume of $100 \mu$, containing (final concentration) $0.1 \mathrm{M}$ potassium phosphate, $\mathrm{pH} 7.4,1 \mathrm{mM}$ ascorbic acid, S9 fractions, and quercetin added from a 200 times concentrated stock solution in DMSO. The final protein content was 0.5 or $0.1 \mathrm{mg} / \mathrm{ml}$ for the incubations with small intestine or liver S9 fractions, respectively. Samples were preincubated at $37^{\circ} \mathrm{C}$ for $1 \mathrm{~min}$ prior to the addition of the cofactor PAPS $(0.1 \mathrm{mM}$, final concentration) to initiate the reaction. The kinetic constants for formation of the sulfate conjugates of quercetin were determined based on incubations performed at substrate concentrations ranging from 0.1 to $4 \mu \mathrm{M}$ quercetin. The reactions were terminated after $12 \mathrm{~min}$ by the addition of $25 \mu \mathrm{l}$ ice-cold acetonitrile:acetic acid (94:6) (v/v). Under these conditions, the formation of sulfate conjugates of quercetin was linear in time and with protein concentration (data not shown). Three replicates of each experiment were performed. Blank incubations were carried out without the cofactor PAPS. Identification of quercetin sulfate conjugates was carried out following UPLC analysis by comparing the UVspectra and the elution order of each metabolite with the study of van der Woude et al. [20]. Formation of the sulfate conjugates of quercetin was indirectly confirmed by treating the sample with sulfatase. To this end, $50 \mu 1$ of a non-terminated mixture was added to $50 \mu 1$ of $0.2 \mathrm{M}$ potassium phosphate, $\mathrm{pH} 6.2$ containing $1 \mathrm{mM}$ ascorbic acid and sulfatase 100 
units $/ \mathrm{ml}$. These samples were incubated for $1 \mathrm{~h}$ at $37^{\circ} \mathrm{C}$ and the reactions were terminated by addition of $25 \mu \mathrm{l}$ ice-cold acetonitrile:acetic acid (94:6) (v/v). Samples were immediately analyzed by UPLC as described in section 2.2.5.

\subsubsection{Methylation of quercetin}

To obtain the kinetic constants for methylation of quercetin, in vitro incubations were performed containing (final concentrations) $0.1 \mathrm{M}$ potassium phosphate, $\mathrm{pH} 7.4,1 \mathrm{mM}$ ascorbic acid, $1 \mathrm{mg} / \mathrm{ml}$ small intestine or liver S9 fractions (male Sprague-Dawley rat), and quercetin added from a 200 times concentrated stock solution in DMSO (100 $\mu$ l final volume). The reactions were initiated by the addition of the cofactor SAM ( $2 \mathrm{mM}$, final concentration) after $1 \mathrm{~min}$ preincubation at $37^{\circ} \mathrm{C}$. Upon incubating for $5 \mathrm{~min}, 25 \mu \mathrm{l}$ ice-cold acetonitrile:acetic acid (94:6) (v/v) were added to terminate the reaction. The kinetic constants were determined using substrate concentrations ranging from 10 to $80 \mu \mathrm{M}$ quercetin. Under these conditions, methylation of quercetin was linear in time and with protein concentration (data not shown). Three replicates of each experiment were performed. Blank incubations were carried out without the cofactor. The methyl conjugates of quercetin were identified following UPLC analysis by comparing the UV-spectra and the retention time to the commercially available standard compounds isorhamnetin (3'-O-methylquercetin) and tamarixetin (4'-O-methylquercetin). Samples were immediately analyzed by UPLC as described in section 2.2.5.

\subsubsection{UPLC analysis}

The amount of glucuronide, sulfate and methyl conjugates formed in the different incubations was quantified using UPLC-DAD analysis. The UPLC system consisted of a Waters (Milford, MA) Acquity binary solvent manager, sample manager, and photodiode array detector, equipped with a Waters Acquity UPLC BEH RP 18 column (1.7 $\mu \mathrm{m}, 2.1$ x 50 $\mathrm{mm})$. Before analysis, all samples were centrifuged at $18600 \mathrm{xg}$ for $5 \mathrm{~min}$ at $5^{\circ} \mathrm{C}$ to precipitate proteins using a VWR microcentrifuge (Model CT15RE, Amsterdam, The Netherlands). Aliquots of $3.5 \mu \mathrm{l}$ of the supernatant obtained from the methylation incubations or aliquots of $10 \mu \mathrm{l}$ of the supernatant obtained from the glucuronidation or sulfation incubations were immediately analyzed. The gradient consisted of acetonitrile and nanopure water containing $0.1 \%$ TFA and a flow rate of $0.6 \mathrm{ml} / \mathrm{min}$ was used. A gradient was applied from $20 \%$ acetonitrile to $25 \%$ acetonitrile over $1.8 \mathrm{~min}$, to $35 \%$ over $0.5 \mathrm{~min}$, keeping the percentage of acetonitrile at $35 \%$ for $0.5 \mathrm{~min}$, followed by an increase to $80 \%$ over $0.2 \mathrm{~min}$. 


\section{Chapter 2}

This percentage was maintained for 1 min before decreasing to $0 \%$ over 0.2 min, keeping the percentage of acetonitrile at $0 \%$ for $1.3 \mathrm{~min}$, followed by increasing the percentage of acetonitrile to reach initial conditions and keeping it at these conditions for $1.1 \mathrm{~min}$.

Another UPLC method was employed to obtain a complete separation of quercetin-7O-glucuronide and quercetin-3-O-glucuronide. This method was less sensitive than the method described above, therefore it was only used to estimate the ratio between these two metabolites. The eluents consisted of acetonitrile and $2.5 \mathrm{mM}$ ammonium acetate in nanopure water. At a flow rate of $0.6 \mathrm{ml} / \mathrm{min}$, a gradient was applied from $10 \%$ acetonitrile to $25 \%$ acetonitrile over $1.8 \mathrm{~min}$, to $90 \%$ over $0.7 \mathrm{~min}$, keeping it at this percentage for $1.3 \mathrm{~min}$ before returning to the starting condition over $0.2 \mathrm{~min}$.

Quercetin metabolites were quantified by integrating the peak areas at $370 \mathrm{~nm}$. Concentrations of quercetin metabolites formed in incubations were quantified using the average of three calibration curves obtained with the commercially available quercetin-3-Oglucuronide, isorhamnetin (3'-O-methylquercetin), and tamarixetin (4'-O-methylquercetin).

\subsubsection{Kinetic analysis}

Kinetic parameters (i.e. apparent maximum velocity $\left(\mathrm{V}_{\max (\mathrm{app})}\right)$ and apparent Michaelis-Menten constant $\left.\left(\mathrm{K}_{\mathrm{m}(\mathrm{app})}\right)\right)$ for glucuronidation, sulfation, and methylation of quercetin were determined by fitting the data to the standard Michaelis-Menten equation:

$$
\mathrm{v}=\mathrm{V}_{\max } *[\mathrm{~S}] /\left(\mathrm{K}_{\mathrm{m}}+[\mathrm{S}]\right)
$$

in which $[\mathrm{S}]$ represents the substrate concentration. Fitting the data to the Michaelis-Menten equation was performed using GraphPad Prism, version 5.04 (GraphPad Software, San Diego, California, USA). The values of $\mathrm{V}_{\max }$ and $\mathrm{K}_{\mathrm{m}}$ were expressed in $\mathrm{nmol} / \mathrm{min} / \mathrm{mg} \mathrm{S} 9$ protein and $\mu \mathrm{M}$, respectively.

\subsubsection{PBK model}

All kinetic parameters obtained were integrated in a PBK model describing the absorption, distribution, metabolism, and excretion (ADME) of quercetin and its metabolites in rats. The model was defined to include separate compartments for blood, liver, kidney, small intestine, lumen, rapidly perfused tissues (e.g. heart, lung, brain), and slowly perfused tissues (e.g. skin, muscle, bone) as shown in Figure 2.2A. Orally ingested quercetin-4'-Oglucoside was hydrolyzed to quercetin aglycone in the intestinal lumen with a clearance of $0.03 \mathrm{l} / \mathrm{h}$. This value was calculated according to the method described by Mihara et al. [28] by using the data obtain from an in situ perfusion of quercetin-4'-O-glucoside in rat small 
intestine at steady-state concentrations [29]. In short, the luminal clearance of quercetin-4'-Oglucoside was calculated as follows:

$$
\mathrm{CL}_{\mathrm{Lu}}=\left(\mathrm{Q}_{\mathrm{p}} *\left(\mathrm{C}_{\mathrm{in}}-\mathrm{C}_{\text {out }}\right)\right) / \mathrm{C}_{\mathrm{in}}
$$

in which, $Q_{p}$ is the perfusion flow rate $(1 \mathrm{ml} / \mathrm{min}), C_{i n}$ represents the concentration of quercetin-4'-O-glucoside before perfusion of $50 \mu \mathrm{M}$, and $\mathrm{C}_{\mathrm{out}}$ is the concentration of quercetin-4'-O-glucoside after passing through the intestinal loop of $23.5 \mu \mathrm{M}$.

A. mono-conjugates

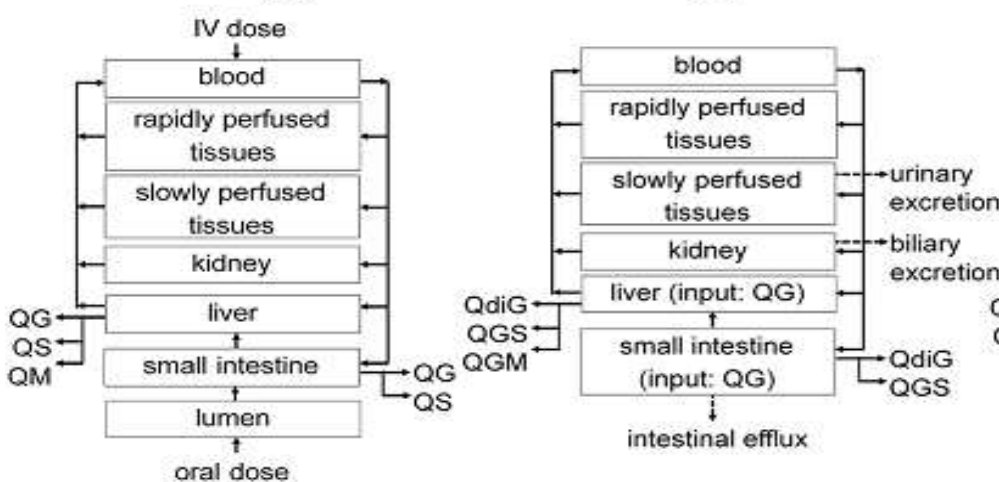

C. tri-conjugates

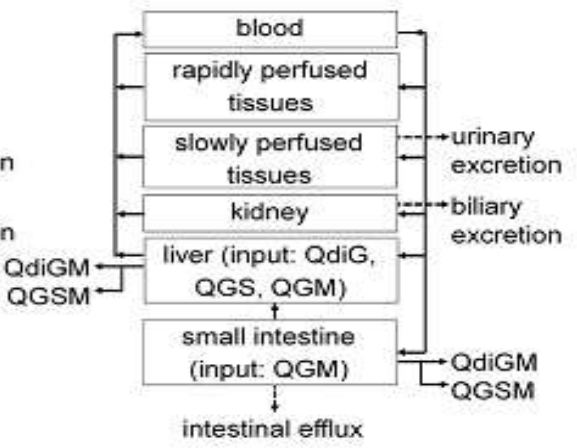

Figure 2.2 Schematic diagram of the PBK model for mono-conjugates (A), di-conjugates (B), and triconjugates $(\mathrm{C})$ of quercetin.

The uptake of quercetin to the small intestine compartment was described by firstorder kinetics with an absorption rate constant of $5.32 \mathrm{~h}^{-1}$ as reported by Chen et al. [30]. As a first step the in vitro kinetic data were integrated in the PBK model to simulate systemic bioavailability of quercetin aglycone and the percentage of formation of different monoconjugates of quercetin. Incubations with small intestine S9 fractions and relevant cofactors resulted in the formation of four mono-conjugates of quercetin: quercetin-7-O-glucuronide, quercetin-3-O-glucuronide, quercetin-3'-O-glucuronide, and quercetin-3'-O-sulfate (see section 2.3.1-2.3.3), which are thus described for this organ. Seven mono-conjugates of quercetin were observed in the incubations with liver S9 fractions and relevant cofactors: quercetin-7-O-glucuronide, quercetin-3'-O-glucuronide, quercetin-4'-O-glucuronide, quercetin-7-O-sulfate, quercetin-3'-O-sulfate, 3'-O-methylquercetin, and 4'-Omethylquercetin (see section 2.3.1-2.3.3) and thus described for this compartment. The kinetic constants for the formation of these metabolites were determined in vitro in the present study (see section 2.3.4). The apparent in vitro $\mathrm{V}_{\max }$ values for glucuronidation, sulfation, and methylation were scaled to in vivo $\mathrm{V}_{\max }$ values using a $\mathrm{S} 9$ protein yield of $11.4 \mathrm{mg} / \mathrm{g}$ small intestine [31] or $143 \mathrm{mg} / \mathrm{g}$ liver [32] as a scaling factor. With the obtained model, the systemic bioavailability (BIO) of quercetin aglycone was determined based on the following equation:

$$
\mathrm{BIO}(\%)=\text { Dose }(\text { i.v. }) / \text { Dose }(\text { oral }) * A U C(\text { oral }) / A U C(\text { i.v. }) * 100
$$




\section{Chapter 2}

in this equation, $\mathrm{AUC}_{0-24 \mathrm{~h}}$ (oral dose) is the area under the concentration-time curve of the blood compartment after an oral dose of quercetin and $\mathrm{AUC}_{0-24 \mathrm{~h}}$ (i.v. dose) is the area under the concentration-time curve of the blood compartment after an intravenous dose of quercetin. In the model, intravenous dose was applied in the blood compartment over a time period of 10 sec.

In a second step the model was adjusted to also include a description of di- and triconjugations and excretion of the different metabolites via bile, urine, and intestinal efflux transport. From the first model, quercetin was predicted to mainly converted to quercetin monoglucuronides (QG) (see section 2.3.5) and thus in a second step further refinement of the model for further conjugation of the monoglucuronides to di- and tri-conjugates was simulated. All monoglucuronides were assumed to be subject to further glucuronidation (at the 3-, 7-, 4'- or 3'- position), sulfation (at the 7- or 3'- position) and/or methylation (at the 4'or $3^{\prime}$ - position) when these positions were still available. The regioselectivity for further conjugation was considered to be the same as observed with the formation of monoconjugates. The 5-position is not a site for conjugation of quercetin [33]. Conjugation was assumed to only occur at different positions on the flavonol ring and not on a functional group that already contains a conjugate [22, 34]. Based on this approach, the formation of different combinations of quercetin diglucuronides (QdiG), quercetin glucuronide sulfate conjugates (QGS), and quercetin glucuronide methyl conjugates (QGM) were described in the model. The di-conjugates were assumed to be subject to further conjugation forming two major types of tri-conjugates, including quercetin diglucuronide methyl conjugates(QdiGM) and quercetin glucuronide sulfate methyl conjugates(QGSM) as these metabolites have been reported in vivo [21]. QGSM conjugates can be formed from either sulfation of QGM or methylation of QGS, and QdiGM can be formed from glucuronidation of QGM or methylation of QdiG. Other theoretically possible tri-conjugates, such as for example quercetin diglucuronide sulfates, were not included in the model because these have not been observed in plasma of rats in vivo and are therefore considered unlikely to be formed. Taking the regiospecifity of possible conjugations into account, a total of 34 different mono-, di-, and tri-conjugates were included in the model.

Metabolism, distribution and excretion of the different mono-, di- and tri-conjugates were described in separate submodels for each metabolite. Each of these submodels consisted of different compartments for blood, liver, kidney, small intestine, rapidly perfused tissues (e.g. heart, lung, brain), and slowly perfused tissues (e.g. skin, muscle, bone) as shown in Figure 2.2B and Figure 2.2C describing for di- and tri-conjugates. Excretion was considered 
to occur in the liver via bile, in the kidney via urine, or in the in the small intestine by active transporters (i.e. P-glycoprotein, breast cancer resistance protein and multidrug resistance proteins) $[35,36]$. The quercetin aglycone was not modeled to be excreted since the aglycone has not been detected in urine and bile in rats following oral uptake of quercetin [30] or in lumen of in situ perfusion of rat small intestine with quercetin [37]. The excretion parameters were considered to be the same for all metabolites and were obtained by fitting the plasma $\mathrm{AUC}_{0-5 \mathrm{~h}}$ of the total metabolites to literature derived $\mathrm{AUC}_{0-5 \mathrm{~h}}$ levels of all metabolites in plasma of rats exposed to $7.6 \mathrm{mg} / \mathrm{kg}$ bw $\left[2-{ }^{14} \mathrm{C}\right]$ quercetin- 4 '-O-glucoside [21]. Conversion of the blood concentrations of the different metabolites to plasma concentrations was done by dividing the blood concentrations by a blood/plasma ratio (R). For all metabolites a $\mathrm{R}$ value of 0.55 (1-hematocrit) was assumed, since most metabolites of quercetin are acidic molecules (see $p \mathrm{~K}_{\mathrm{a}}$ values Table 2.2) [38]. Based on this approach, the biliary and the urinary excretion rate constants were estimated to be 16 and $6.5 \mathrm{~h}^{-1}$, and the intestinal efflux clearance of the metabolites to the lumen was estimated to be $0.17 \mathrm{l} / \mathrm{h}$. Finally, enterohepatic circulation of quercetin and its metabolites has not been included in the model as Chen et al. reported the absence of significant enterohepatic circulation of quercetin and its metabolites in rats [30].

The kinetic constants for formation of the 30 different di- and tri-conjugates were estimated by fitting the predicted plasma $\mathrm{AUC}_{0-5 \mathrm{~h}}$ of QG, QdiG, QGS, QGM, QdiGM, and QGSM to literature derived $\mathrm{AUC}_{0-5 \mathrm{~h}}$ levels of these metabolites [21]. Further conjugation of monoglucuronides was simulated under the assumption that the catalytic efficiency for further conjugation was lower than observed for the formation of the different mono-conjugates, due to both an increase in $\mathrm{K}_{\mathrm{m}}$ and a decrease in $\mathrm{V}_{\max }$. This assumption was validated with an in vitro experiment in which quercetin-3-O-glucuronide was used as a substrate. Although the exact kinetic constants for further conjugation of quercetin-3-O-glucuronide could not be determined due to low catalytic efficiencies of the different reactions, both the affinity and capacity of the reactions seemed to decrease (data not shown). The factors by which the $\mathrm{K}_{\mathrm{m}}$ and the $\mathrm{V}_{\max }$ values for di- and tri-conjugates with glucuronide, sulfate, or methyl moiety were affected, as determined by fitting to the experimental data, are shown in Table 2.1.

The physiological parameters and the tissue:blood partition coefficients used in the model are given in Table 2.1 and Table 2.2, respectively. The physiological parameters were obtained from Brown et al. [39]. The tissue:blood partition coefficients of quercetin and its metabolites were estimated from $\log K_{\text {ow }}$ according to a method of DeJongh et al. [40]. Log $K_{\text {ow }}$ values were estimated from $\log P$ value obtained from Scifider (american Chemical Society, USA) for quercetin and its mono-conjugates or $C \log P$ values obtained from 
ChemBio3D Ultra 2010 (CambridgeSoft, USA) for di- and tri-conjugates (Table 2.2). The derived partition coefficients are around one, which can be expected based on the low lipophilicity of quercetin and its metabolites [41]. Only the partition coefficients of $3^{\prime}-\mathrm{O}-$ methylquercetin and 4'-O-methylquercetin are slightly higher due to a higher lipophilicity of these metabolites [42]. Mass balance and model equations were coded and numerically integrated in Berkeley Madonna 8.3.18 (Macey and Oster, UC Berkeley, CA) using the Rosenbrock's algorithm for stiff system. The parameters for excretion of metabolites and factors by which $\mathrm{K}_{\mathrm{m}}$ and $\mathrm{V}_{\max }$ for formation of di-and tri-conjugates were affected, were obtained by using the multiple-curve fitting function in Berkeley Madonna, which minimizes the root mean square deviation between the reported in vivo data points and the model output. When using this function, increasing in the number of parameters to be fitted also increase the risk that the program will find a local minimum of residual instead of the global minimum [43]. To prevent this to happen, several different starting values were set. Furthermore, by limiting the values of the parameters to physiologically plausible values, the estimation of unrealistic parameters can be prevented [43]. With the model obtained, predictions were made on circulating metabolites in plasma specifying the conjugation position. The model equations for quercetin and its metabolites are described as follows:

\section{Amount of quercetin in tissues and blood}

Small intestine: $\mathrm{dAI}_{\mathrm{Q}} / \mathrm{dt}=\mathrm{K}_{\mathrm{a}} * \mathrm{ALu}_{\mathrm{Q}}+\mathrm{QI} *\left(\mathrm{CB}_{\mathrm{Q}}-\mathrm{CI}_{\mathrm{Q}} / \mathrm{PI}_{\mathrm{Q}}\right)$

$$
\begin{aligned}
& -\mathrm{V}_{\text {max }, I_{-} \text {Mmono }} * \mathrm{CI}_{\mathrm{Q}} / \mathrm{PI}_{\mathrm{Q}} /\left(\mathrm{K}_{\mathrm{m}, \mathrm{I}_{-} \text {Mmono }}+\mathrm{CI}_{\mathrm{Q}} / \mathrm{PI}_{\mathrm{Q}}\right) \\
\mathrm{CI}_{\mathrm{Q}}= & \mathrm{AI}_{\mathrm{Q}} / \mathrm{VI}
\end{aligned}
$$

Liver: $\quad \mathrm{dAL} / \mathrm{dt}=\quad \mathrm{QI}^{*} \mathrm{CI}_{\mathrm{Q}} / \mathrm{PI}_{\mathrm{Q}}+\mathrm{QL} *\left(\mathrm{CB}_{\mathrm{Q}}-\mathrm{CL}_{\mathrm{Q}} / \mathrm{PL}_{\mathrm{Q}}\right)$

$$
\begin{aligned}
& -\mathrm{V}_{\text {max }, \mathrm{L} \_ \text {Mmono }} * \mathrm{CL}_{\mathrm{Q}} / \mathrm{PL}_{\mathrm{Q}} /\left(\mathrm{K}_{\mathrm{m}, \mathrm{L}_{-} \mathrm{M} \text { mono }}+\mathrm{CL}_{\mathrm{Q}} / \mathrm{PL}_{\mathrm{Q}}\right) \\
\mathrm{CL}_{\mathrm{Q}}= & \mathrm{AL}_{\mathrm{Q}} / \mathrm{VL}
\end{aligned}
$$

Blood:

$$
\mathrm{CK}_{\mathrm{Q}}=\mathrm{AK} \mathrm{Q}_{\mathrm{Q}} / \mathrm{VK}
$$

where $\mathrm{CTi}_{\mathrm{Q}}$ (in $\mu \mathrm{mol} / \mathrm{l}$ ) is the concentration of quercetin in tissue ( $\mathrm{Ti}=\mathrm{Lu}$ (lumen), I (small intestine), L (liver), or K (kidney), R (rapidly perfused tissues), or S (slowly perfused tissues), $\mathrm{ATi}_{\mathrm{Q}}$ (in $\mu \mathrm{mol}$ ) is the amount of quercetin in a tissue, $\mathrm{AB}_{\mathrm{Q}}\left(\right.$ in $\mu \mathrm{mol}$ ) and $\mathrm{CB}_{\mathrm{Q}}$ (in $\mu \mathrm{mol} / \mathrm{l}$ ) are the amount and the concentration of quercetin in blood, QTi (in $1 / \mathrm{h}$ ) is the blood flow to a 
tissue, and $\mathrm{PTi}_{\mathrm{Q}}$ is the tissue:blood partition coefficient of quercetin. Mmono refers to either of the specified mono-conjugates as described in Table 2.3 and Figure 2.2.

Amount of quercetin mono-conjugate in tissues and blood

Small intestine: $\mathrm{dAI}_{\mathrm{Mmono}} / \mathrm{dt}=\mathrm{QI} *\left(\mathrm{CB}_{\mathrm{Mmono}}-\mathrm{CI}_{\mathrm{Mmono}} / \mathrm{PI}_{\mathrm{Mmono}}\right)$

$$
\begin{aligned}
& +\mathrm{V}_{\text {max }, \text { I_Mmono }} * \mathrm{CI}_{\mathrm{Q}} / \mathrm{PI}_{\mathrm{Q}} /\left(\mathrm{K}_{\mathrm{m}, \mathrm{I} \_\mathrm{Mmono}}+\mathrm{CI}_{\mathrm{Q}} / \mathrm{PI}_{\mathrm{Q}}\right) \\
& -\mathrm{V}_{\text {max }, \mathrm{I} \_\mathrm{Mdi}} * \mathrm{CI}_{\mathrm{Mmono}} / \mathrm{PI}_{\mathrm{Mmono}} /\left(\mathrm{K}_{\mathrm{m}, \mathrm{I} \_\mathrm{Mdi}}+\mathrm{CI}_{\mathrm{Mmono}} / \mathrm{PI}_{\mathrm{Mmono}}\right) \\
& -\mathrm{CL}_{\mathrm{Ief}} *\left(\mathrm{CI}_{\mathrm{Mmono}} / \mathrm{PI}_{\mathrm{Mmono}}\right) \\
\mathrm{CI}_{\mathrm{Mmono}} & =\mathrm{AI}_{\mathrm{Mmono}} / \mathrm{VI}
\end{aligned}
$$

Liver: $\quad \mathrm{dAL}_{\mathrm{Mmono}} / \mathrm{dt}=\mathrm{QI}^{*} \mathrm{CI}_{\mathrm{Mmono}} / \mathrm{PI}_{\mathrm{Mmono}}+\mathrm{QL}^{*} \mathrm{CB}_{\mathrm{Mmono}}$

$$
\begin{aligned}
& -(\mathrm{QI}+\mathrm{QL}) * \mathrm{CL}_{\mathrm{Mmono}} / \mathrm{PL}_{\mathrm{Mmono}} \\
& +\mathrm{V}_{\text {max,L_Mmono }} * \mathrm{CL}_{\mathrm{Q}} / \mathrm{PL}_{\mathrm{Q}} /\left(\mathrm{K}_{\mathrm{m}, \mathrm{L} \_\mathrm{M} \text { mono }}+\mathrm{CL}_{\mathrm{Q}} / \mathrm{PL}_{\mathrm{Q}}\right) \\
& -\mathrm{V}_{\text {max,L_Mdi }} * \mathrm{CL}_{\mathrm{Mmono}} / \mathrm{PL}_{\mathrm{Mmono}} /\left(\mathrm{K}_{\mathrm{m}, \mathrm{L} \_\mathrm{M} \text { di }}+\mathrm{CL}_{\mathrm{Mmono}} / \mathrm{PL}_{\mathrm{Mmono}}\right) \\
& -\mathrm{K}_{\mathrm{bile}}{ }^{*} \mathrm{AL}_{\mathrm{Mmono}} \\
\mathrm{CL}_{\mathrm{Mmono}} & =\mathrm{AL}_{\mathrm{Mmono}} / \mathrm{VL}
\end{aligned}
$$

Kidney: $\quad \mathrm{dAK}_{\mathrm{Mmono}} / \mathrm{dt}=\mathrm{QK} *\left(\mathrm{CB}_{\mathrm{Mmono}}-\mathrm{CK}_{\mathrm{Mmono}} / \mathrm{PK}_{\mathrm{Mmono}}\right)$

$$
\begin{array}{r}
-\mathrm{K}_{\text {urine }} * \mathrm{AK}_{\text {Mmono }} \\
\mathrm{CK}_{\text {Mmono }}=\mathrm{AK}_{\text {Mmono }} / \mathrm{VK}
\end{array}
$$

Blood: $\quad \mathrm{dAB}_{\mathrm{Mmono}} / \mathrm{dt}=(\mathrm{QI}+\mathrm{QL}) * \mathrm{CL}_{\mathrm{Mmono}} / \mathrm{PL}_{\mathrm{Mmono}}+\mathrm{QK}^{*} \mathrm{CK}_{\mathrm{Mmono}} / \mathrm{PK}_{\mathrm{Mmono}}$

$$
\begin{aligned}
+ & \mathrm{QR} * \mathrm{CR}_{\mathrm{Mmono}} / \mathrm{PR}_{\mathrm{Mmono}}+\mathrm{QS} * \mathrm{CS}_{\mathrm{Mmono}} / \mathrm{PS}_{\mathrm{Mmono}} \\
- & \mathrm{QC} * \mathrm{CB}_{\mathrm{Mmono}} \\
\mathrm{CB}_{\text {Mmono }} & =\mathrm{AB}_{\mathrm{Mmono}} / \mathrm{VB}
\end{aligned}
$$$$
\mathrm{CP}_{\text {Mmono }}=\mathrm{CB}_{\text {Mmono }} / \mathrm{R}
$$

where, $\mathrm{CTi}_{\mathrm{Mmono}}$ (in $\left.\mu \mathrm{mol} / \mathrm{l}\right)$ is the concentration of mono-conjugate metabolite in tissue $(\mathrm{Ti}=$ I (small intestine), L (liver), K (kidney), R (rapidly perfused tissues), or S (slowly perfused tissues), $\mathrm{ATi}_{\text {Mmono }}$ (in $\mu \mathrm{mol}$ ) is the amount of mono-conjugate metabolite in a tissue, $\mathrm{AB}_{\text {Mmono }}$ (in $\mu \mathrm{mol}$ ) and $\mathrm{CB}_{\mathrm{Mmono}}$ (in $\mu \mathrm{mol} / \mathrm{l}$ ) are the amount and the concentration of mono-conjugate metabolite in blood, QTi (in $1 / \mathrm{h}$ ) is the blood flow to a tissue, $\mathrm{PTi}_{\mathrm{Mmono}}$ is the tissue:blood partition coefficient of mono-conjugate, and $\mathrm{CP}_{\mathrm{Mmono}}$ (in $\mu \mathrm{mol} / \mathrm{l}$ ) are the concentration of mono-conjugate metabolite in plasma. $\mathrm{R}$ is the blood/plasma ratio. Mmono and Mdi refer to either of the specified mono-conjugates or di-conjugates as described in Table 2.3 and Figure 2.2 . 


\section{Amount of quercetin di-and tri-conjugate in tissues and blood}

The amount of quercetin di-and tri-conjugates in tissues and blood were described in the model using the same equations as described for mono-conjugates (equation 12-19). The equations used to describe the formation of di-and tri-conjugates of quercetin in the small intestine and liver included the factors by which the $\mathrm{K}_{\mathrm{m}}$ and the $\mathrm{V}_{\max }$ values for di- and triconjugates with glucuronide, sulfate, or methyl moiety were affected, and are described as follows:

$$
\begin{aligned}
& \text { sulfation of QG }=\mathrm{V}_{\max , T i \_M m o n o} / \mathrm{a}^{*} \mathrm{CTi}_{\mathrm{Mmono}} / \mathrm{PTi}_{\mathrm{Mmono}} \\
& /\left(\mathrm{K}_{\mathrm{m}, \mathrm{Ti} \_\mathrm{Mmono}} * \mathrm{~d}+\mathrm{CTi}_{\mathrm{Mmono}} / \mathrm{PTi}_{\mathrm{Mmono}}\right) \\
& \text { sulfation of QGM } \quad=\mathrm{V}_{\text {max }, T i}{ }_{\text {Mmono }} / \mathrm{a}^{*} \mathrm{CTi}_{\text {Mdi }} / \mathrm{PTi}_{\mathrm{Mdi}} \\
& /\left(\mathrm{K}_{\mathrm{m}, \mathrm{Ti}} \text { Mmono } * \mathrm{~d}+\mathrm{CTi}_{\mathrm{Mdi}} / \mathrm{PTi}_{\mathrm{Mdi}}\right) \\
& \text { methylation of QG } \quad=\mathrm{V}_{\max , T i \_M m o n o} / \mathrm{b}^{*} \mathrm{CTi}_{\mathrm{Mmono}} / \mathrm{PTi}_{\mathrm{Mmono}} \\
& /\left(\mathrm{K}_{\mathrm{m}, \mathrm{Ti} \_ \text {Mmono }} * \mathrm{e}+\mathrm{CTi}_{\mathrm{Mmono}} / \mathrm{PTi}_{\mathrm{Mmono}}\right) \\
& \text { methylation of QGS } \quad=\mathrm{V}_{\max , T i \_M m o n o} / \mathrm{b}^{*} \mathrm{CTi}_{\mathrm{Mdi}_{\mathrm{i}}} / \mathrm{PTi}_{\mathrm{Mdi}} \\
& /\left(\mathrm{K}_{\mathrm{m}, \mathrm{Ti} \_ \text {Mmono }} * \mathrm{e}+\mathrm{CTi}_{\mathrm{Mdi}} / \mathrm{PT}_{\mathrm{Mdi}}\right) \\
& \text { methylation of QdiG } \quad=\mathrm{V}_{\max , T i} \mathrm{Mmono}_{3} / \mathrm{b}_{3} * \mathrm{CTi}_{\mathrm{Mdi}} / \mathrm{PTi}_{\mathrm{Mdi}} \\
& /\left(\mathrm{K}_{\mathrm{m}, \mathrm{Ti}} \text { Mmono } * \mathrm{e}_{3}+\mathrm{CTi}_{\mathrm{Mdi}} / \mathrm{PT}_{\mathrm{Mdi}}\right) \\
& \text { glucuronidation of QG } \quad=\mathrm{V}_{\text {max,Ti_Mmono }} / \mathrm{c}^{*} \mathrm{CTi}_{\text {Mmono }} / \mathrm{PTi}_{\text {Mmono }} \\
& /\left(\mathrm{K}_{\mathrm{m}, \mathrm{Ti}} \text { Mmono } * \mathrm{f}+\mathrm{CTi}_{\text {Mmono }} / \mathrm{PI}_{\text {Mmono }}\right) \\
& \text { glucuronidation of QGM }=\mathrm{V}_{\text {max,Ti_Mmono }} / \mathrm{c}^{*} \mathrm{CTi}_{\mathrm{Mdi}} / \mathrm{PTi}_{\mathrm{Mdi}} \\
& /\left(\mathrm{K}_{\mathrm{m}, \mathrm{Ti}} \mathrm{Mmono} / \mathrm{f}+\mathrm{CTi}_{\mathrm{Mdi}} / \mathrm{PI}_{\mathrm{Mdi}}\right)
\end{aligned}
$$

Where CTi (in $\mu \mathrm{mol}$ ) and PTi is the concentration and the tissue:blood partition coefficient of mono-conjugate (Mmono), di-conjugate (Mdi), or tri-conjugate (Mtri) in tissue. The reduction factors for the $\mathrm{V}_{\max }$ values and the induction factors for $\mathrm{K}_{\mathrm{m}}$ valuesfor formation of di- and triconjugates, as obtained by fitting the plasma $\mathrm{AUC}_{0-5 \mathrm{~h}}$ of the total metabolites to literature derived $\mathrm{AUC}_{0-5 \mathrm{~h}}$ levels of all metabolites, are presented in Table 2.1.

\subsection{Sensitivity analysis}

A sensitivity analysis was performed to assess which model parameters contribute substantially to a specific model output. Normalized sensitivity coefficients (SCs) were calculated for the model parameters according to the method described by Evens and Andersen [44] as follows:

$$
\mathrm{SC}=\left(\mathrm{C}^{\prime}-\mathrm{C}\right) /\left(\mathrm{P}^{\prime}-\mathrm{P}\right)^{*}(\mathrm{P} / \mathrm{C})
$$


in this equation, $C$ is the initial value of the model output, $C^{\prime}$ is the modified value of the model output resulting from an increase in parameter value, $P$ is the initial parameter value, and $P^{\prime}$ is the modified parameter value assuming a $5 \%$ increase in its value.

Sensitivity analyses were carried out for the plasma $\mathrm{AUC}_{0-5 \mathrm{~h}}$ of mono-, di- and triconjugates of quercetin at dose of $7.6 \mathrm{mg} / \mathrm{kg}$ bw quercetin-4'-O-glucoside; representing the dose that was applied in the literature reported in vivo study that was used in the present study for parameterization of the model.

Table 2.1 Physiological parameters used in the PBK model for quercetin in rats

\begin{tabular}{|c|c|c|c|}
\hline \multirow[t]{2}{*}{ Parameters } & \multirow[t]{2}{*}{ Symbol } & \multicolumn{2}{|c|}{ Value } \\
\hline & & Literature & Fitted in vivo \\
\hline Body weight (kg) [39] & BW & 0.25 & \\
\hline \multicolumn{4}{|l|}{ Tissue volumes (\% body weight) [39] } \\
\hline lumen & $\mathrm{VLu}$ & 0.5 & \\
\hline small intestine & VI & 1.4 & \\
\hline liver & VL & 3.4 & \\
\hline kidney & VK & 0.7 & \\
\hline rapidly perfused tissues & VR & 3.2 & \\
\hline slowly perfused tissues & VS & 74.6 & \\
\hline blood & VB & 7.4 & \\
\hline Cardiac output (1/h) [39] & QC & 5.4 & \\
\hline \multicolumn{4}{|l|}{ Blood flow to tissue ( $\%$ cardiac output) [39] } \\
\hline small intestine & QI & 15.1 & \\
\hline liver (exclude portal vein) & QL & 9.9 & \\
\hline kidney & QK & 14.1 & \\
\hline rapidly perfused tissues & $\mathrm{QR}$ & 36.9 & \\
\hline slowly perfused tissues & QS & 24 & \\
\hline Absorption rate constant of quercetin $\left(\mathrm{h}^{-1}\right)[30]$ & $\mathrm{K}_{\mathrm{a}}$ & 5.32 & \\
\hline Biliary excretion rate constant $\left(\mathrm{h}^{-1}\right)$ & $\mathrm{K}_{\text {bile }}$ & & 16 \\
\hline Urinary excretion rate constant $\left(\mathrm{h}^{-1}\right)$ & $\mathrm{K}_{\text {urine }}$ & & 6.5 \\
\hline Intestinal efflux clearance $(1 / h)$ & $\mathrm{CL}_{\mathrm{Ief}}$ & & 0.17 \\
\hline Luminal clearance of quercetin-4'-O-glucoside (1/h) [29] & $\mathrm{CL}_{\mathrm{Lu}}$ & 0.03 & \\
\hline \multicolumn{4}{|l|}{ Reduction factor for $\mathrm{V}_{\max }$} \\
\hline sulfation of QG or QGM & a & & 1.5 \\
\hline methylation of QG or QGS & $\mathrm{b}$ & & 1 \\
\hline glucuronidation of QG or QGM & $\mathrm{c}$ & & 12 \\
\hline methylation of QdiG & $b_{3}$ & & 31 \\
\hline \multicolumn{4}{|l|}{ Induction factor for $\mathrm{K}_{\mathrm{m}}$} \\
\hline sulfation of QG or QGM & $\mathrm{d}$ & & 2.2 \\
\hline methylation of QG or QGS & $\mathrm{e}$ & & 1 \\
\hline glucuronidation of QG or QGM & $\mathrm{f}$ & & 13 \\
\hline methylation of QdiG & $e_{3}$ & & 87 \\
\hline
\end{tabular}


Table 2.2 Physico-chemical parameters for quercetin and its metabolites ${ }^{1}$

\begin{tabular}{|c|c|c|c|c|c|c|c|}
\hline Compound & $\log P^{2}$ & $p$ Ka & $\begin{array}{c}\text { Small } \\
\text { intestine }\end{array}$ & Liver & Kidney & $\begin{array}{c}\text { Rapidly } \\
\text { perfused } \\
\text { tissue }\end{array}$ & $\begin{array}{c}\text { Slowly } \\
\text { perfused } \\
\text { tissue }\end{array}$ \\
\hline quercetin & 2.0 & 6.3 & 1.3 & 1.3 & 1.5 & 1.5 & 0.6 \\
\hline quercetin-7-O-glucuronide & -0.5 & 2.7 & 0.8 & 0.8 & 0.8 & 0.8 & 0.4 \\
\hline quercetin-3-O-glucuronide & 0.6 & 2.8 & 0.9 & 0.9 & 0.9 & 0.9 & 0.5 \\
\hline quercetin-3'-O-glucuronide & -0.003 & 2.8 & 0.8 & 0.8 & 0.8 & 0.8 & 0.4 \\
\hline quercetin-4'-O-glucuronide & -0.1 & 2.8 & 0.8 & 0.8 & 0.8 & 0.8 & 0.4 \\
\hline quercetin-7-O-sulfate & 1.2 & -4.9 & 1.0 & 1.0 & 1.0 & 1.0 & 0.5 \\
\hline quercetin-3'-O-sulfate & 1.2 & -4.4 & 1.0 & 1.0 & 1.0 & 1.0 & 0.5 \\
\hline 3'-O-methylquercetin & 2.8 & 6.3 & 2.0 & 2.0 & 2.6 & 2.6 & 0.7 \\
\hline 4'-O-methylquercetin & 2.7 & 6.3 & 1.8 & 1.8 & 2.4 & 2.4 & 0.7 \\
\hline quercetin diglucuronides & -2.7 & & 0.7 & 0.7 & 0.8 & 0.8 & 0.4 \\
\hline $\begin{array}{l}\text { quercetin glucuronide sulfate } \\
\text { conjugates }\end{array}$ & -2.2 & - & 0.7 & 0.7 & 0.8 & 0.8 & 0.4 \\
\hline $\begin{array}{l}\text { quercetin glucuronide methyl } \\
\text { conjugates }\end{array}$ & -0.2 & - & 0.8 & 0.8 & 0.8 & 0.8 & 0.4 \\
\hline $\begin{array}{l}\text { quercetin diglucuronide methyl } \\
\text { conjugates }\end{array}$ & -2.3 & - & 0.7 & 0.7 & 0.8 & 0.8 & 0.4 \\
\hline $\begin{array}{l}\text { quercetin glucuronide sulfate } \\
\text { methyl conjugates }\end{array}$ & -1.8 & - & 0.8 & 0.8 & 0.8 & 0.8 & 0.4 \\
\hline
\end{tabular}

\subsection{Results}

\subsubsection{Glucuronidation of quercetin}

UPLC analysis of incubations with rat small intestine and liver S9 fractions in the presence of quercetin and UDPGA reveals the formation of different metabolites with retention times of $0.52,0.82$ and $0.95 \mathrm{~min}$. Treatment of the samples with $\beta$-glucuronidase resulted in complete disappearance of these peaks and a concomitant equivalent increase in the peak area of quercetin (data not shown). The chromatographic profile was comparable to that reported in the literature for the same reaction, enabling assignment of the peaks in our chromatograms according to the identifications reported by Boersma et al. [19]. This assignment was supported by an additional analysis of collected metabolites from the UPLC by HPLC using the same method as described by Boersma et al. [19] (data not shown). Based on this comparison, the metabolites with a retention time of 0.82 and 0.95 min were ascribed to quercetin-4'-O-glucuronide and quercetin-3'-O-glucuronide, whereas the peak with a retention time of 0.52 min was considered to contain a mixture of quercetin-3- and -7-Oglucuronide. To determine the relative quantity of each of these metabolites in this peak, the samples were further analyzed with a different UPLC method that allowed separation of the two peaks (see Section 2.2.5). These analyses revealed that for incubations with rat intestine S9 the peak at 0.52 min contained $20 \%$ quercetin-3-O-glucuronide and $80 \%$ quercetin-7-O- 
glucuronide. This ratio was consistent at different concentrations. In incubations with rat liver S9 fractions, the peak at $0.52 \mathrm{~min}$, was identified to only contain quercetin-7-O-glucuronide. Figure 2.3 shows the rate formation of the different quercetin monoglucuronides obtained with the incubations with male rat liver (A) or small intestine S9 fractions (B) with increasing in concentrations of quercetin.

liver $\mathbf{5 9}$

A. glucuronidation

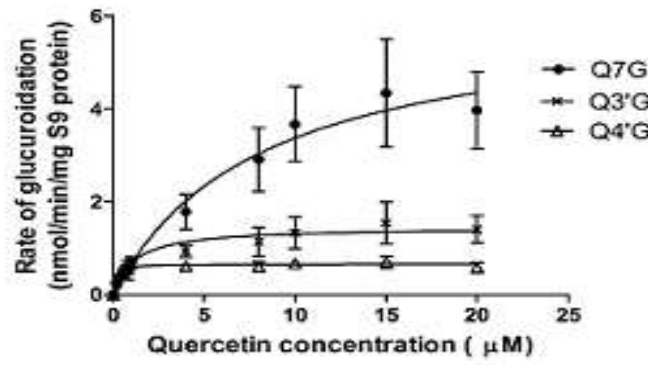

C. sulfation

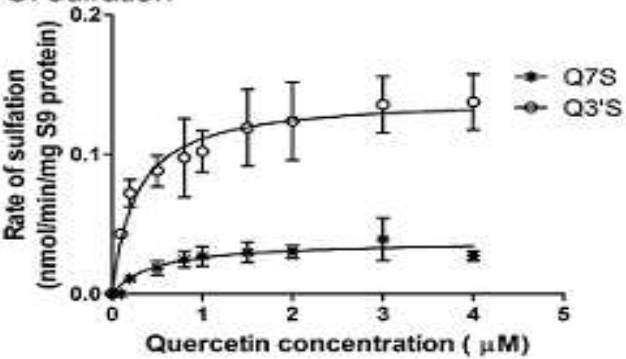

E. methylation

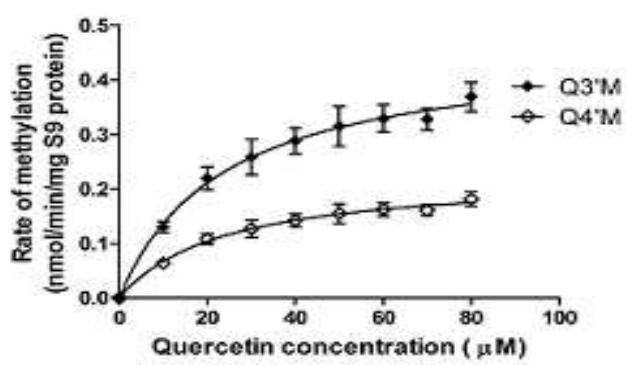

small intestine $\mathbf{S 9}$

B. glucuronidation

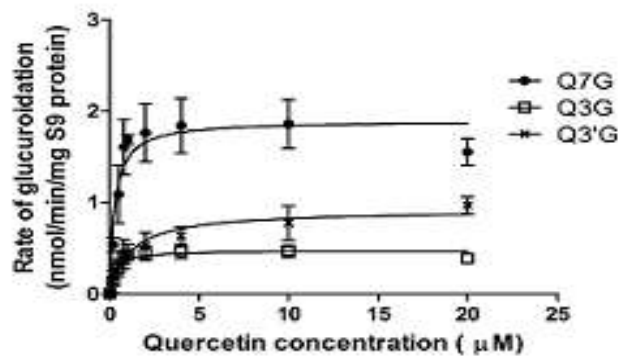

D. sulfation

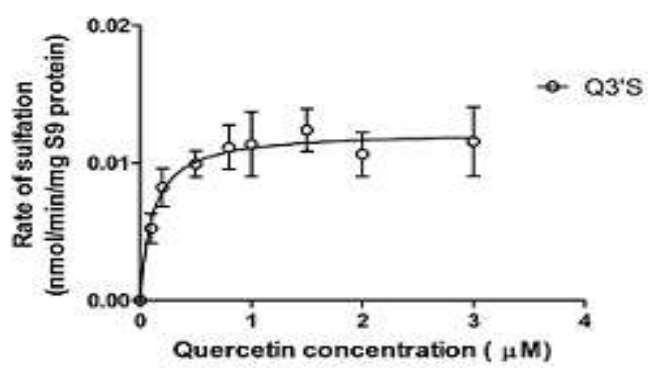

Figure 2.3 Quercetin concentration dependent formation of different glucuronide conjugates by male rat liver S9 (A) or small intestinal S9 (B), different sulfate conjugates by male rat liver S9 (C) or intestinal S9 (D), and different methyl conjugates by male rat liver S9 (E); quercetin (Q), glucuronide $(\mathrm{G})$, sulfate (S), and methylate (M).

\subsubsection{Sulfation of quercetin}

UPLC chromatogram of incubations with rat small intestine S9 fractions in the presence of quercetin and PAPS as cofactor reveals the formation of one metabolite with a retention time of $1.09 \mathrm{~min}$, while incubations with rat liver S9 fractions result in two metabolites with retention times of 0.71 and $1.09 \mathrm{~min}$. Treatment of the samples with 


\section{Chapter 2}

sulfatase result in complete elimination of these metabolites and a concomitant equivalent increase in the amount of quercetin (data not shown). The chromatographic profile was comparable to that reported in the literature for the same reaction by van der Woude et al. [20]. The chromatographic profile of sulfate conjugates of quercetin obtained from the UPLC analysis was comparable to what has previously been reported by van der Woude et al. [20] enabling assignment of the peaks in our chromatograms according to the identifications reported by van der Woude et al. [20]. This assignment was supported by an additional analysis of collected metabolites from the UPLC by HPLC using the same method as described by van der Woude et al. [20] (data not shown). The peaks at 0.71 and 1.09 min could thus be ascribed to quercetin-7-O-sulfate and quercetin-3'-O-sulfate, respectively. The quercetin concentration dependent rate of formation of these metabolites in incubations with rat liver or small intestine $\mathrm{S} 9$ fractions is shown in Figure 2.3C and Figure 2.3D.

\subsubsection{Methylation of quercetin}

UPLC chromatogram of incubations with rat small intestine or liver S9 fractions in the presence of SAM as cofactor reveals that there was no methylation of quercetin by small intestine S9 fractions, whereas two metabolites were formed in incubations with liver S9 fractions with a retention time of 2.49 and $2.52 \mathrm{~min}$. The metabolite with a retention time of 2.49 min coeluted with commercially available 3'-O-methylquercetin and was thus identified as $3^{\prime}$-O-methylquercetin. The one with a retention of $2.52 \mathrm{~min}$ was found to coelute with commercially available 4'-O-methylquercetin and was therefore identified as 4'-Omethylquercetin. The quercetin concentration dependent rate of formation of the methylquercetin conjugates by rat liver $\mathrm{S} 9$ fractions is depicted in Figure 2.3E.

\subsubsection{Kinetic constants for formation of different quercetin mono-conjugates}

The kinetic constants for formation of mono-conjugates of quercetin with a glucuronide, sulfate, or methyl moiety were obtained by fitting the data to a standard Michaelis-Menten equation (Table 2.3). The in vitro $\mathrm{V}_{\max }$ values obtained with S9 from different organs were scaled to in vivo $\mathrm{V}_{\max }$ values using scaling factors (see section 2.2.7). The in vivo catalytic efficiencies for formation of the different mono-conjugates of quercetin reveal that liver is the major site for metabolism of quercetin as the catalytic efficiencies for all reactions in this tissue are higher than the catalytic efficiencies for all reactions in small intestine. Glucuronidation is the most efficient pathway and quercetin-4'-O-glucuronide is the 
major mono-conjugate formed. The high catalytic efficiency for formation of quercetin-4'-Oglucuronide is mainly due to a relatively low $\mathrm{K}_{\mathrm{m}}$ value for this reaction.

Table 2.3 Kinetic parameters for conjuguation of quercetin in incubations with small intestine and liver $\mathbf{S} 9$ fractions from male Sprague-Dawley rats

\begin{tabular}{|c|c|c|c|c|c|c|}
\hline Organ & Metabolite & $\begin{array}{c}\mathrm{Km}(\mathrm{app}) \\
(\mu \mathrm{M})\end{array}$ & $\begin{array}{c}\text { Vmax(app) } \\
\text { (nmol/min/mg } \\
\text { /S9 protein) }\end{array}$ & $\begin{array}{c}\text { In vitro catalytic } \\
\text { efficiency } \\
(\mu 1 / \text { min } / m g S 9 \\
\text { protein) }\end{array}$ & $\begin{array}{c}\text { Scaled } \\
\text { Vmax, in } \\
\text { vivo } \\
(\mu \mathrm{mol} / \mathrm{h})^{1}\end{array}$ & $\begin{array}{c}\text { In vivo } \\
\text { catalytic } \\
\text { efficiency } \\
\text { (1/h) }\end{array}$ \\
\hline \multirow{4}{*}{$\begin{array}{l}\text { Small } \\
\text { intestine }\end{array}$} & quercetin-7-O-glucuronide & 0.3 & 1.9 & 6.3 & 4.5 & 15 \\
\hline & quercetin-3-O-glucuronide & 0.3 & 0.5 & 1.7 & 1.2 & 4.0 \\
\hline & quercetin-3'-O-glucuronide & 1.1 & 0.9 & 0.8 & 2.2 & 2.0 \\
\hline & quercetin-3'-O-sulfate & 0.1 & 0.01 & 0.1 & 0.03 & 0.3 \\
\hline \multirow{7}{*}{ Liver } & quercetin-7-O-glucuronide & 8.1 & 6.1 & 0.8 & 445 & 55 \\
\hline & quercetin-3'-O-glucuronide & 1.1 & 1.5 & 1.4 & 109 & 99 \\
\hline & quercetin-4'-O-glucuronide & 0.1 & 0.7 & 7.0 & 51 & 511 \\
\hline & quercetin-7-O-sulfate & 0.5 & 0.04 & 0.1 & 2.9 & 5.8 \\
\hline & quercetin-3'-O-sulfate & 0.3 & 0.1 & 0.3 & 7.3 & 24 \\
\hline & 3'-O-methylquercetin & 24 & 0.5 & 0.02 & 36 & 1.5 \\
\hline & 4'-O-methylquercetin & 24 & 0.2 & 0.01 & 15 & 0.6 \\
\hline
\end{tabular}

${ }^{1}$ Small intestine: Vmax (app)/(1000 nmol/umol)* $(60 \mathrm{~min} / \mathrm{hr}) *(11.4 \mathrm{mg} \mathrm{S9} / \mathrm{g} \text { intestine })^{*}(14 \mathrm{~g} \text { intestine/kg bw })^{*}(0.25 \mathrm{~kg}$ bw), Liver: $\operatorname{Vmax}(\mathrm{app}) /(1000 \mathrm{nmol} / \mathrm{umol}) *(60 \mathrm{~min} / \mathrm{hr}) *(143 \mathrm{mg} \mathrm{S} 9 / \mathrm{g}$ liver $) *(34 \mathrm{~g}$ liver/kg bw $) *(0.25 \mathrm{~kg} \mathrm{bw})$.

2.3.5 Integrating the kinetic data to predict the bioavailability of quercetin aglycone and formation of mono-conjugate metabolites

As a first step the kinetic constants obtained were integrated in a PBK model to predict systemic bioavailability of quercetin in rats upon exposure to quercetin in its aglycone form or to quercetin-4'-O-glucoside and to provide an indication of the percentage of the different mono-conjugates of quercetin that are formed. The model predicted that the bioavailability of quercetin when dosed as aglycone is very low, amounting to $0.004 \%$ at a dose relevant to dietary intake $(0.4 \mathrm{mg} / \mathrm{kg}$ bw quercetin aglycone) and $0.1 \%$ at a dose level representing supplementary intake (12 mg/kg bw quercetin aglycone). Quercetin-4'-O-glucoside was predicted to have the same level of bioavailability at low dose, but 3.3-fold lower at high dose, amounting to 0.004 or $0.03 \%$ when providing the same level of quercetin equivalents by dosing of $0.6 \mathrm{mg} / \mathrm{kg}$ bw or $19 \mathrm{mg} / \mathrm{kg}$ bw quercetin-4'-O-glucoside.

When dosed as aglycone, quercetin was completely metabolized within $1.5 \mathrm{~h}$ and was almost exclusively converted to monoglucuronides accounting to 99 to $95 \%$ of the dose at a dose ranging from 0.1 to $50 \mathrm{mg} / \mathrm{kg}$ bw. Sulfation and methylation only represented minor metabolic pathways with 0.9 to $4.5 \%$ of the dose being converted via sulfation and 0.01 to 


\section{Chapter 2}

$0.8 \%$ of the dose being converted via methylation at different oral doses of quercetin. When quercetin was dosed as 4'-O-glucoside complete metabolism was observed within $2.5 \mathrm{~h}$ and 99 to $97 \%$ of the dose was converted to monoglucuronides at a dose ranging from 0.1 to 50 $\mathrm{mg} / \mathrm{kg}$ bw. Figure 2.4 presents a plot of the percentage of the dose that is converted into the different monoglucuronides at different oral dose levels of quercetin aglycone (Figure 2.4A) or quercetin-4'-O-glucoside (Figure 2.4B). At low dose level of quercetin $(0.4 \mathrm{mg} / \mathrm{kg}$ bw quercetin aglycone), $68 \%$ of the dose is predicted to be converted to quercetin-7-Oglucuronide, $18 \%$ to quercetin-3-O-glucuronide, $10 \%$ to quercetin-3'-O-glucuronide, and $3.1 \%$ quercetin-4'-O-glucuronide. Formation of 7- and 3-O-glucuronidated metabolites thus represent the primary metabolic reactions at low doses. However, as the dose increases the relative contribution of these metabolites decreases, which is accompanied by a relative increase in formation of $3^{\prime}$-and 4'-O-glucuronide. At a dose of $12 \mathrm{mg} / \mathrm{kg}$ bw approximately $39 \%$ of the dose is predicted to be converted to quercetin-4'-O-glucuronide, $31 \%$ to quercetin7-O-glucuronide, $20 \%$ to quercetin-3'-O-glucuronide, and 6.6\% to quercetin-3-O-glucuronide. For quercetin-4'-O-glucoside, this shift in metabolism occurred at higher dose levels. These results suggest that regiospecificity of metabolites will depend on the dose of quercetin and whether it is absorbed as a glucoside or not.
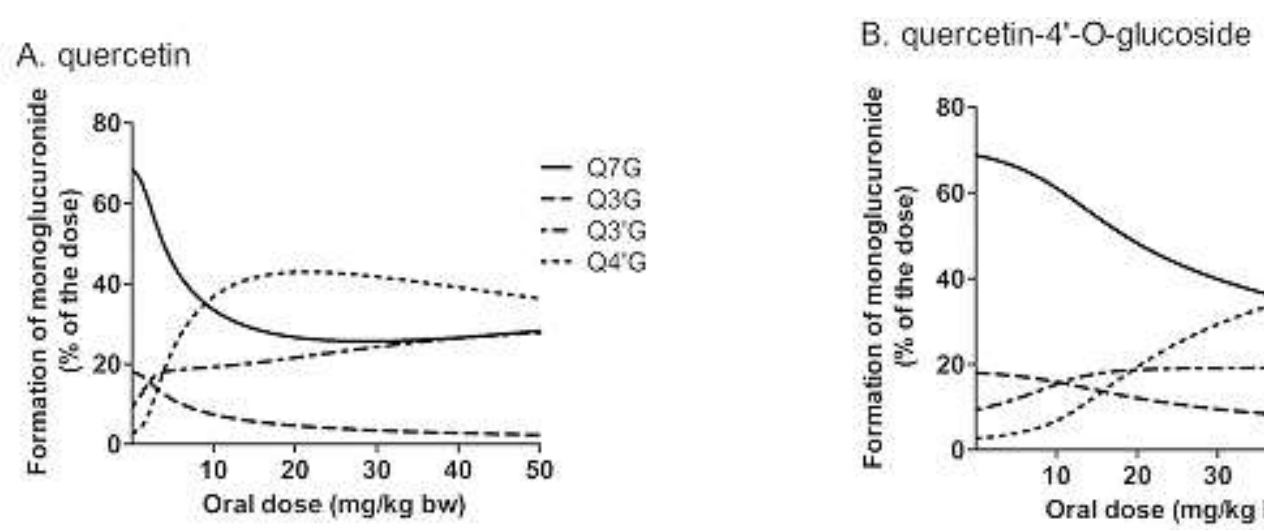

Figure 2.4 PBK model based prediction on dose-dependent formation of quercetin-7-O-glucuronide $(\mathrm{Q} 7 \mathrm{G})$, quercetin-3-O-glucuronide $(\mathrm{Q} 3 \mathrm{G})$, quercetin-3'-O-glucuronide $\left(\mathrm{Q} 3{ }^{\prime} \mathrm{G}\right)$, and quercetin-4'-Oglucuronide $\left(\mathrm{Q} 4^{\prime} \mathrm{G}\right)$ in rat orally exposed to quercetin (A) or quercetin-4'-O-glucoside (B). Data are expressed as percentage of the dose.

2.3.6 Adjustment of the model to predict plasma concentrations of mono-, di- and triconjugates

Given that 95 to $99 \%$ of quercetin and quercetin-4'-O-glucoside is converted to different monoglucuronides after $1.5 \mathrm{~h}$ to $2.5 \mathrm{~h}$ and the fact di- and tri-conjugates have been observed in the plasma of rats in vivo [21, 22], we considered that all monoglucuronides that 
are formed are subjected to further conjugation. In a second step, we have therefore included the further conjugation of quercetin monoglucuronides into di- and tri-conjugates in the model as well as urinary, biliary, and intestinal excretion of the different metabolites. This enabled prediction of the plasma concentrations of the various mono-, di- and tri-conjugates. The kinetic constants for further conjugation and urinary, biliary, and intestinal excretion were obtained by fitting the predicted plasma $\mathrm{AUC}_{0-5 \mathrm{~h}}$ of different metabolites to the $\mathrm{AUC}_{0-5 \mathrm{~h}}$ values that were derived from in vivo reported plasma concentrations of the same metabolites in rats exposed to $7.6 \mathrm{mg} / \mathrm{kg}$ bw $\left[2-{ }^{14} \mathrm{C}\right]$ quercetin-4'-O-glucoside as described by Graf et al. [21]. Figure $2.5 \mathrm{~A}$ shows the best fit that was obtained, which allowed to predict the $\mathrm{AUC}_{0-5 \mathrm{~h}}$ of these metabolites in plasma within 0.9 to 3.9-fold from the reported levels [21]. In addition, the model obtained allowed to adequately predict results from a second study in which rats were exposed to $7.6 \mathrm{mg} / \mathrm{kg}$ bw $\left[2-{ }^{14} \mathrm{C}\right]$ quercetin-4'-O-glucoside as reported by Mullen et al . [22] (Figure 2.5B). Plasma concentrations of different metabolites at $1 \mathrm{~h}$ after exposure were observed to be within 19 to 3 -fold of the observed plasma concentrations.

A.

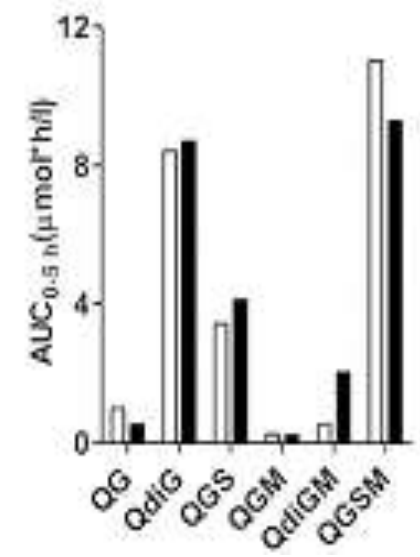

B.

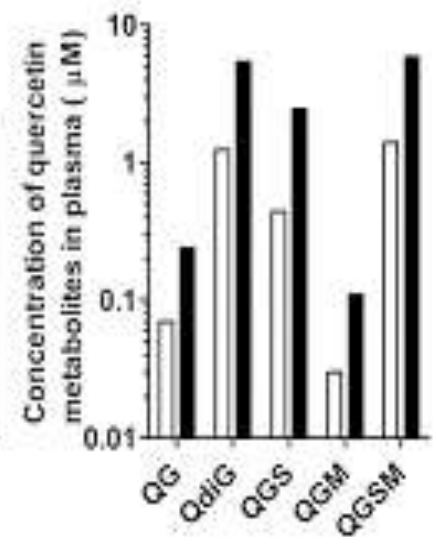

Figure 2.5 Comparison of the predicted plasma $\mathrm{AUC}_{0-5 \mathrm{~h}}$ levels of quercetin metabolites (black bar) to literature derived $\mathrm{AUC}_{0-5 \mathrm{~h}}$ levels of these metabolites by Graf et al. [21] (white bar) (A) and comparison of the predicted amount of quercetin metabolites in plasma at $1 \mathrm{~h}$ (black bar) to the reported in vivo levels of these metabolites by Mullen et al. [22] (white bar) of rat exposed to $7.6 \mathrm{mg} / \mathrm{kg}$ bw quercetin-4'O-glucoside; quercetin monoglucuronides (QG), quercetin diglucuronides (QdiG), quercetin glucuronide methyl conjugates (QGM), quercetin glucuronide sulfate conjugates (QGS), quercetin diglucuronide methyl conjugates (QdiGM), and quercetin glucuronide sulfate methyl conjugates (QGSM) (B).

Apart from simulating total concentrations of the different major circulating metabolites the model also allowed to tentatively predict the regiospecificity of the conjugation for the most important circulating metabolites. The regiospecificity of the ten metabolites with the highest $\mathrm{AUC}_{0-5 \mathrm{~h}}$ at a dose level ranging from 0.1 to $50 \mathrm{mg} / \mathrm{kg} \mathrm{bw}$ quercetin and quercetin-4'-O-glucoside is shown in Figure 2.6A and Figure 2.6B. Together these metabolites contributed to approximately 71 to $86 \%$ of the total plasma $\mathrm{AUC}_{0-5 \mathrm{~h}}$. At a low dose of quercetin $(0.4 \mathrm{mg} / \mathrm{kg} \mathrm{bw}), \mathrm{Q} 7 \mathrm{G} 3{ }^{\prime} \mathrm{S} 4^{\prime} \mathrm{M}$ is predicted to be the major circulating 
metabolites, followed by Q74'diG', and Q7G3'S accounting to 27, 16, and 10\% total plasma $\mathrm{AUC}_{0-5 \mathrm{~h}}$. However, as the dose increase the relative contribution of these metabolites decreases, which is accompanied by a relative increase in plasma concentrations of Q3'4'diG, Q4'G3'S, Q74'diG3'M, and Q4'G7S3'M. For quercetin-4'-O-glucoside, the predicted regiospecificity of the major circulating metabolites is similar to what is predicted for quercetin, but the shift in the metabolism happened at higher dose levels.

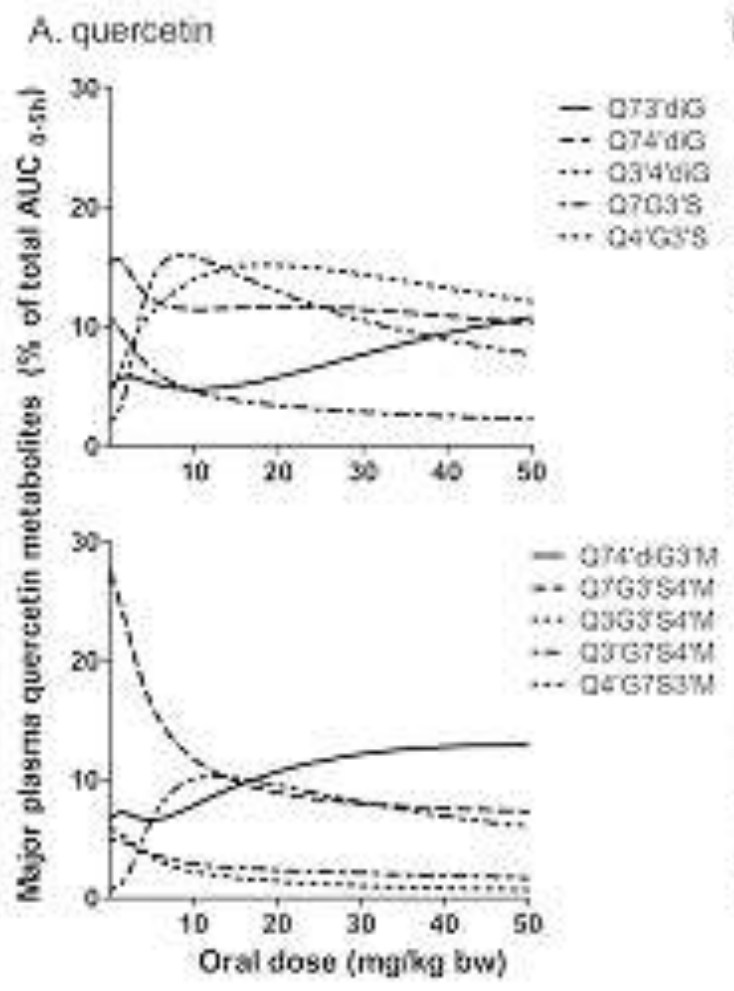

8. quercetin-4-O-glucoside

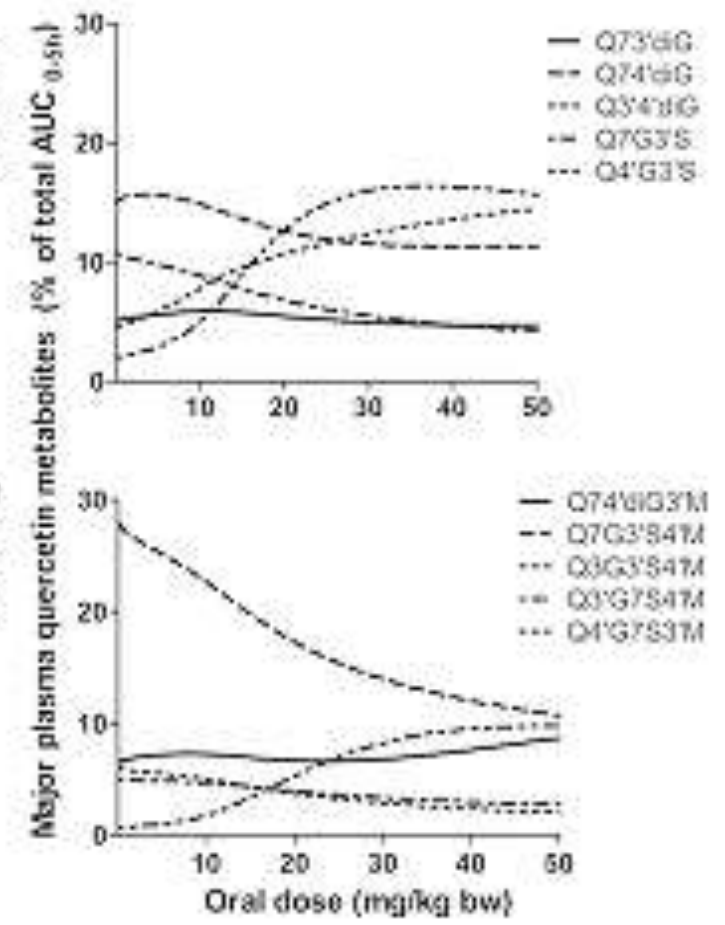

Figure 2.6 PBK model predicted dose-dependent regiospecificity of the major circulating metabolites of quercetin in plasma of rat orally exposed to quercetin (A) or quercetin-4'-O-glucoside (B); quercetin $(\mathrm{Q})$, glucuronide $(\mathrm{G})$, sulfate $(\mathrm{S})$, methylate $(\mathrm{M})$. Data were expressed as percentage of the total plasma $\mathrm{AUC}_{0-5 \mathrm{~h}}$.

\subsubsection{Sensitivity analysis}

A sensitivity analysis was performed to assess the key parameters that influence the predicted plasma $\mathrm{AUC}_{0-5 \mathrm{~h}}$ of quercetin metabolites at a dose of $7.6 \mathrm{mg} / \mathrm{kg}$ bw quercetin-4'-Oglucoside. Only parameters that had a normalized sensitivity coefficient higher than 0.1 (in absolute value) are shown in Figure 2.7. The results obtained reveal that the $\mathrm{AUC}_{0-5 \mathrm{~h}}$ levels of quercetin metabolites in plasma are mainly influenced by the kinetic constants for formation of quercetin-4'-O-glucuronide and querctin-3'-O-sulfate in the liver as well as factors by which the kinetic constants for formation of di- and tri-conjugates were estimated to be reduced (Table 2.1). The plasma $\mathrm{AUC}_{0-5 \mathrm{~h}}$ levels of methyl containing metabolites are also affected by the kinetic constants for formation of $3^{\prime}$ - and 4'-O-methylquercetin in the liver. 
The kinetic constants for formation of 4'-O-methylquercetin are found to greatly affect the plasma $\mathrm{AUC}_{0-5 \mathrm{~h}}$ levels of QGS. Parameters determining the excretion of quercetin metabolites especially the biliary excretion rate constant and the intestinal efflux clearance as well as the parameter determining plasma concentration ( $\mathrm{R}$ value) are found to predominantly influence the plasma $\mathrm{AUC}_{0-5 \mathrm{~h}}$ levels of quercetin di- and tri-conjugates. Other parameters including physiological parameters especially the body weight and the tissue volumes (i.e. small intestine and liver), and the scaling factor for liver S9 concentration are found to have an impact on the plasma $\mathrm{AUC}_{0-5 \mathrm{~h}}$ levels of all quercetin metabolites. To a minor extent, the plasma $\mathrm{AUC}_{0-5 \mathrm{~h}}$ levels of quercetin metabolites are influenced by the blood flow to tissue (i.e. small intestine and liver). Partition coefficient parameters do not significantly affect the plasma $\mathrm{AUC}_{0-5 \mathrm{~h}}$ levels of the different quercetin metabolites.

\subsection{Discussion}

In vitro tests to elucidate the mechanisms of action are often performed with flavonoid aglycones. Questions remain as in what form the flavonoids are ultimately present in the in vivo systemic circulation. PBK models can be used to answer this question since these models allows to predict time-dependent concentrations of compounds as well as their metabolites in plasma or in tissues. Therefore, in the present study PBK models were developed using quercetin and its glucoside (quercetin-4'-O-glucoside) as model compound to predict plasma concentrations of quercetin and its metabolites.

Bioavailability of quercetin as aglycone was estimated to range between $0.004 \%$ to $0.1 \%$ at dose levels of $0.4 \mathrm{mg} / \mathrm{kg}$ bw and $12 \mathrm{mg} / \mathrm{kg}$ bw quercetin or quercetin equivalent when dosing as 4'-O-glucoside (0.6 and $19 \mathrm{mg} / \mathrm{kg}$ bw). In most in vivo studies no quercetin aglycone or only a trace amount of the aglycone have been detected in plasma of rats upon oral exposure to quercetin or quercetin-4'-O-glucoside [13, 21, 45], supporting the low bioavailability of quercetin. One study did report quercetin concentrations in plasma and estimated an oral bioavailability of quercetin in rats of $5.3 \%$ at an oral dose of $10 \mathrm{mg} / \mathrm{kg}$ bw quercetin [30]. This value is somewhat higher than the predicted level with the PBK model, but confirms that the plasma concentrations of quercetin aglycone will be limited.

Glucuronidation was found to be the major pathway responsible for the low bioavailability of quercetin aglycone, since as much as 95 to $99 \%$ of the dose was predicted to be converted to monoglucuronides within $1.5 \mathrm{~h}$ to $2.5 \mathrm{~h}$ at different oral doses of quercetin or quercetin-4'-O-glucoside (Figure 2.4). This is in line with the study of Mullen et al. as $73.8 \%$ of quercetin was metabolized within 1 hour at a dose of $7.6 \mathrm{mg} / \mathrm{kg}$ bw $\left[2-{ }^{14} \mathrm{C}\right]$ quercetin- $4{ }^{\prime}-\mathrm{O}-$ 
glucoside, primarily resulting in metabolites that contain a glucuronide [22]. An additional level of evaluation of the model performance may be obtained by comparison of the
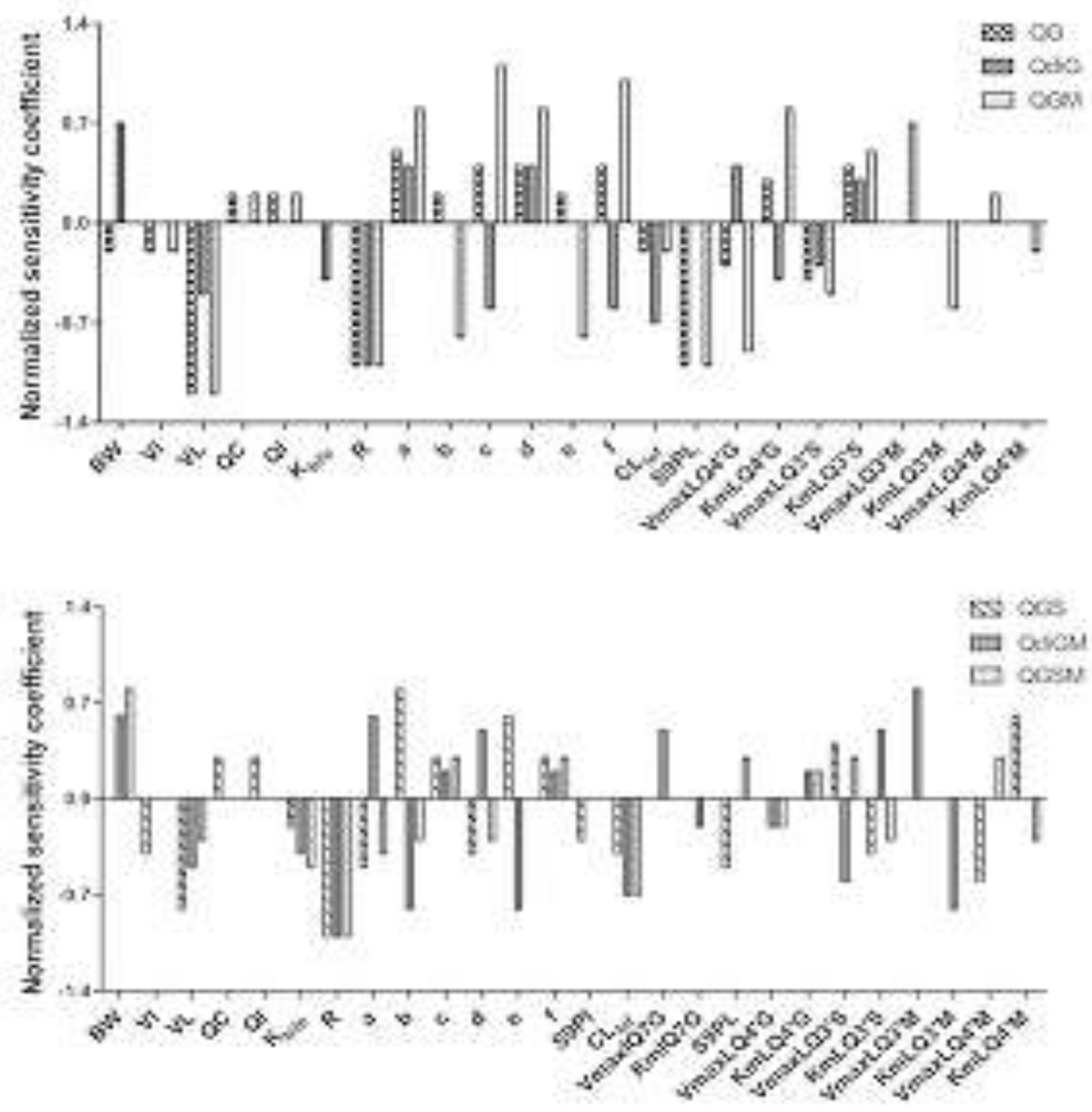

Figure 2.7 Sensitivity analysis of the predicted plasma $\mathrm{AUC}_{0-5 \mathrm{~h}}$ of quercetin monoglucuronides $(\mathrm{QG})$, quercetin diglucuronides (QdiG), quercetin glucuronide methyl conjugates (QGM), quercetin glucuronide sulfate conjugates (QGS), quercetin diglucuronide methyl conjugates (QdiGM), and quercetin glucuronide sulfate methyl conjugates (QGSM) in rat exposed to $7.6 \mathrm{mg} / \mathrm{kg}$ bw quercetin-4'O-glucoside. The parameters are stand for: $\mathrm{bw}=$ body weight, $\mathrm{VTi}=$ tissue volume $(\mathrm{Ti}=\mathrm{I}$ (small intestine), $\mathrm{L}$ (liver)), $\mathrm{QC}=$ cardiac output, $\mathrm{QI}=$ blood flow to small intestine, $\mathrm{K}_{\mathrm{bile}}=$ biliary excretion rate constant, $\mathrm{R}=$ blood/plasma ratio, $\mathrm{CL}_{\mathrm{Ief}}=$ intestinal efflux clearance, $\mathrm{S} 9 \mathrm{PI}=$ small intestinal $\mathrm{S} 9$ protein content, $\mathrm{S} 9 \mathrm{PL}=$ liver $\mathrm{S} 9$ protein content, $\mathrm{V}_{\max }$ and $\mathrm{K}_{\mathrm{m}}=$ the maximum rate of formation and the Michaelis-Menten constant for formation of metabolite ( $\mathrm{G}$ (glucuronide), $\mathrm{S}$ (sulfate), M (methylate), reduction factor of $\mathrm{V}_{\max }$ for sulfate-conjugate of QG or QGM (a), methylate-conjugate of QG or QGS (b), glucuronide-conjugate of QG or QGM (c), and induction factor of $\mathrm{K}_{\mathrm{m}}$ for sulfateconjugate of QG or QGM (d), methylate-conjugate of QG or QGS (e), glucuronide-conjugate of QG or QGM (f). 
predicted excretion of quercetin metabolites via bile, urine and intestinal efflux transport to in vivo reported levels of excretion through these routes. The excretion parameters for the model defined were obtained by fitting the predicted $\mathrm{AUC}_{0-5 \mathrm{~h}}$ of the total quercetin metabolites in plasma to the reported levels in vivo. Based on this approach, the model predicted that the amount of quercetin metabolites excreted to bile after $24 \mathrm{~h}$ corresponds to $35 \%$ of the dose at a dose of $10 \mathrm{mg} / \mathrm{kg}$ bw quercetin, which is the same as the reported level in rats of $35 \%$ of the dose at the same dose level [30]. The predicted urinary excretion of quercetin metabolites after $24 \mathrm{~h}$, corresponding to $3 \%$ of the dose at a dose of $49 \mathrm{mg} / \mathrm{kg}$ bw quercetin-4'-Oglucoside, is only 4-fold lower than the level reported in rats exposed to an equivalent dose quercetin-3-O-glucoside which amounted to $12 \%$ of the dose [46]. The predicted intestinal efflux of quercetin metabolites after $24 \mathrm{~h}$, amounting to $63 \%$ of the dose at a dose of 0.9 $\mathrm{mg} / \mathrm{kg}$ bw quercetin, is only 1.2-fold higher than the level of $52 \%$ of the dose reported for rats exposed to the same dose [37]. Altogether, the model adequately predicted biliary and urinary excretion as well as intestinal efflux of quercetin metabolites, indicating that the highest level of excretion occurs via intestinal efflux transporters.

Due to the fast level of formation of monoglucuronides, these metabolites can be subjected to further metabolism resulting in the formation of di- and tri-conjugates of quercetin. The kinetic parameters for all possible di- and tri-conjugates were obtained by fitting these parameters to match in vivo reported plasma concentrations of the different mono-, di-, and tri-conjugates. The results revealed that especially further glucuronidation of quercetin monoglucuronides occurs with a lower catalytic efficiency than glucuronidation of the quercetin itself. This is likely due to an increase in the molecular size of the substrates which could affect their binding to the conjugating enzymes as reported by Takenaga et al. who stated that for indolocarbazole analogs a molecular size smaller than $14.5 \AA$ might be required for a compound to be a substrate for O-glucuronidation [47]. Manevski et al. also reported an effect of the size of substrate on the binding to UGT1A6 with the relatively bulkier size chain of psilocin preventing its binding to the active site of UGT1A6, whereas the somewhat smaller size chain of serotonin allows binding [48]. In addition to further glucuronidation, also methylation of quercetin diglucuronides was also predicted to occur with a much lower catalytic efficiency than methylation of quercetin, which may be related to the bulkiness of the diglucuronide. Other conversions were estimated to differ only slightly compared with the kinetic constants for the formation of mono-conjugates.

Both exposure to quercetin as aglycone and to its 4'-O-glucoside was predicted to result in circulating metabolites of quercetin that are di- and tri-conjugates of 


\section{Chapter 2}

monoglucuronides, especially QdiG and QGSM (Figure 2.6). Already at $0.5 \mathrm{~h}$ upon dosing with $0.4 \mathrm{mg} / \mathrm{kg}$ bw quercetin, their AUC in plasma makes up $64 \%$ of the total quercetin and its metabolites in plasma. This prediction is in agreement with data reported in rats [21-23] as well as in humans $[24,25]$. In rats, various di- and tri-conjugates of quercetin were detected at $0.5 \mathrm{~h}$ upon dosing with $7.6 \mathrm{mg} / \mathrm{kg}$ bw $\left[2-{ }^{14} \mathrm{C}\right]$ quercetin-4'-O-glucuronide, in which QdiG was the major circulating metabolites at $0.5 \mathrm{~h}$ as time passed QGSM became the major metabolites at $1 \mathrm{~h}$ [21]. The same results are also observed in humans, also various di- and triconjugates of quercetin were detected in plasma at $0.5 \mathrm{~h}$ upon dosing with onion $[24,25]$. The biological activity of quercetin or flavonoids in vivo could be due to these circulating metabolites as conjugation of flavonoid does not always result in a reduced biological activity of flavonoids [8]. In addition, it is increasingly postulated that deconjugation of the conjugates may be responsible for the biological activity by releasing the active aglycone in a tissue $[49,50]$. However, so far no in vitro experiments have been performed to identify whether deconjugating enzymes can actually deconjugate di- and tri-conjugates. The results obtained with the PBK model allow to identify which metabolites will be relevant to study for their biological activity or to use in incubation to determine deconjugation, which are predominantly QdiG and QGSM metabolites.

Overall, the newly developed PBK model adequately predicted plasma concentrations of quercetin and its metabolites including mono-, di-, and tri-conjugates and provides an indication of the nature of the circulating metabolites of quercetin in plasma of rat. As we still lack in vivo kinetic data for many flavonoids, the developed model has a great potential to be used as a platform to build PBK models for other flavonoids as well as to predict the kinetics of flavonoids in humans.

\section{Conflict of interest}

There is no conflict of interest.

\section{Acknowledgments}

This research was financially supported by the Ministry of Science and Technology of Thailand through a Royal Thai Government Scholarship awarded to Rungnapa Boonpawa for conducting her PhD thesis in The Netherlands. 
A PBK model for quercetin in rats

\section{References}

1. Neuhouser, M. L., Review: dietary flavonoids and cancer risk: evidence from human population studies. Nutrition and Cancer, 2004. 50(1): p. 1-7.

2. Perez-Vizcaino, F. and J. Duarte, Flavonols and cardiovascular disease. Molecular Aspects of Medicine, 2010. 31(6): p. 478-494.

3. Larson, A., J. D. Symons, and T. Jalili, Quercetin: A treatment for hypertension?-A review of efficacy and mechanisms. Pharmaceuticals, 2010. 3(1): p. 237-250.

4. Aguirre, L., et al., Beneficial effects of quercetin on obesity and diabetes. The Open Nutraceuticals, 2011. 4: p. 189-198.

5. Ahn, J., et al., The anti-obesity effect of quercetin is mediated by the AMPK and MAPK signaling pathways. Biochemical and Biophysical Research Communications, 2008. 373(4): p. 545-549.

6. Heo, H. J. and C. Y. Lee, Protective Effects of Quercetin and Vitamin C against Oxidative Stress-Induced Neurodegeneration. Journal of Agricultural and Food Chemistry, 2004. 52(25): p. 7514-7517.

7. Suematsu, N., M. Hosoda, and K. Fujimori, Protective effects of quercetin against hydrogen peroxide-induced apoptosis in human neuronal SH-SY5Y cells. Neuroscience Letters, 2011. 504(3): p. 223-227.

8. Beekmann, K., et al., A state-of-the-art overview of the effect of metabolic conjugation on the biological activity of flavonoids. Food \& Function, 2012. 3(10): p. 1008-1018.

9. Manthey, J. A., Biological Properties of Flavonoids Pertaining to Inflammation. Microcirculation, 2000. 7(S1): p. S29-S34.

10. Brand, W., et al., Flavonoid-mediated inhibition of intestinal ABC transporters may affect the oral bioavailability of drugs, food-borne toxic compounds and bioactive ingredients. Biomedicine \&amp; Pharmacotherapy, 2006. 60(9): p. 508-519.

11. Havsteen, B. H., The biochemistry and medical significance of the flavonoids. Pharmacology \& Therapeutics, 2002. 96(2-3): p. 67-202.

12. Williamson, G., The use of flavonoid aglycones in in vitro systems to test biological activities: based on bioavailability data, is this a valid approach? 2002. 1(2): p. 215-222.

13. de Boer, V. C. J., et al., Tissue distribution of quercetin in rats and pigs. The Journal of Nutrition, 2005. 135(7): p. 1718-1725.

14. Lee, J. and A. E. Mitchell, Quercetin and isorhamnetin glycosides in onion (Allium cepa L.): varietal comparison, physical distribution, coproduct evaluation, and long-term storage stability. Journal of Agricultural and Food Chemistry, 2011. 59(3): p. 857-863.

15. Németh, K., et al., Deglycosylation by small intestinal epithelial cell $\beta$-glucosidases is a critical step in the absorption and metabolism of dietary flavonoid glycosides in humans. European Journal of Nutrition, 2003. 42(1): p. 29-42.

16. Manach, C., et al., Polyphenols: food sources and bioavailability. The American Journal of Clinical Nutrition, 2004. 79(5): p. 727-747.

17. Gao, S. and M. Hu, Bioavailability challenges associated with development of anti-cancer phenolics. Mini reviews in medicinal chemistry, 2010. 10(6): p. 550-567.

18. Day, A. J., et al., Absorption of quercetin-3-glucoside and quercetin-4'-glucoside in the rat small intestine: the role of lactase phlorizin hydrolase and the sodium-dependent glucose transporter. Biochemical Pharmacology, 2003. 65(7): p. 1199-1206.

19. Boersma, M. G., et al., Regioselectivity of phase II metabolism of luteolin and quercetin by UDP-glucuronosyl transferases. Chemical Research in Toxicology, 2002. 15(5): p. 662-670. 
20. van der Woude, H., et al., Identification of 14 quercetin phase II mono- and mixed conjugates and their formation by rat and human phase II in vitro model systems. Chemical Research in Toxicology, 2004. 17(11): p. 1520-1530.

21. Graf, B. A., et al., Disposition and metabolism of $\left[2-{ }^{14} \mathrm{C}\right]$ quercetin-4'-glucoside in rats. Drug Metabolism and Disposition, 2005. 33(7): p. 1036-1043.

22. Mullen, W., et al., Determination of flavonol metabolites in plasma and tissues of rats by HPLC-radiocounting and tandem mass spectrometry following oral ingestion of [214C]quercetin-4'-glucoside. Journal of Agricultural and Food Chemistry, 2002. 50(23): p. 6902-6909.

23. Graf, B. A., et al., Rat gastrointestinal tissues metabolize quercetin. The Journal of Nutrition, 2006. 136(1): p. 39-44.

24. Lee, J., et al., UHPLC-(ESI)QTOF MS/MS profiling of quercetin metabolites in human plasma postconsumption of applesauce enriched with apple peel and onion. Journal of Agricultural and Food Chemistry, 2012. 60(34): p. 8510-8520.

25. Mullen, W., C. A. Edwards, and A. Crozier, Absorption, excretion and metabolite profiling of methyl-, glucuronyl-, glucosyl- and sulpho-conjugates of quercetin in human plasma and urine after ingestion of onions. British Journal of Nutrition, 2006. 96: p. 107-116.

26. Easterbrook, J., et al., Effects of Organic Solvents on the Activities of Cytochrome P450 Isoforms, UDP-Dependent Glucuronyl Transferase, and Phenol Sulfotransferase in Human Hepatocytes. Drug Metabolism and Disposition, 2001. 29(2): p. 141-144.

27. Fisher, M. B., et al., In vitro glucuronidation using human liver microsomes and the poreforming peptide alamethicin. Drug Metabolism and Disposition, 2000. 28(5): p. 560-566.

28. Mihara, K., et al., Intestinal first-pass metabolism of eperisone in the rat. Pharmaceutical Research, 2001. 18(8): p. 1131-1137.

29. Arts, I. C. W., et al., The type of sugar moiety is a major determinant of the small intestinal uptake and subsequent biliary excretion of dietary quercetin glycosides. British Joural of Nutrition, 2004. 91: p. 841-847.

30. Chen, X., et al., Pharmacokinetics and modeling of quercetin and metabolites. Pharmaceutical Research, 2005. 22(6): p. 892-901.

31. van de Kerkhof, E. G., I. A. M. de Graaf, and G. M. M. Groothuis, In vitro methods to study intestinal drug metabolism. Current Drug Metabolism, 2007. 8(7): p. 658-675.

32. Punt, A., et al., A physiologically based biokinetic (PBBK) model for estragole bioactivation and detoxification in rat. Toxicology and Applied Pharmacology, 2008. 231(2): p. 248-259.

33. Day, A. J., et al., Conjugation position of quercetin glucuronides and effect on biological activity. Free Radical Biology and Medicine, 2000. 29(12): p. 1234-1243.

34. Argikar, U. A., Unusual Glucuronides. Drug Metabolism and Disposition, 2012. 40(7): p. 1239-1251.

35. Sesink, A. L. A., et al., Breast Cancer Resistance Protein (Bcrp1/Abcg2) Limits Net Intestinal Uptake of Quercetin in Rats by Facilitating Apical Efflux of Glucuronides. Molecular Pharmacology, 2005. 67(6): p. 1999-2006.

36. Scheepens, A., K. Tan, and J. Paxton, Improving the oral bioavailability of beneficial polyphenols through designed synergies. 2010. 5(1): p. 75-87.

37. Crespy, V., et al., Part of quercetin absorbed in the small intestine is conjugated and further secreted in the intestinal lumen. American Journal of Physiology - Gastrointestinal and Liver Physiology, 1999. 277(1): p. G120-G126.

38. Cubitt, H., J. B. Houston, and A. Galetin, Relative importance of intestinal and hepatic glucuronidation -impact on the prediction of drug clearance. 2009. 26(5): p. 1073-1083. 
39. Brown, R. P., et al., Physiological parameter values for physiologically based pharmacokinetic models. Toxicology and Industrial Health, 1997. 13(4): p. 407-484.

40. DeJongh, J., H. J. M. Verhaar, and J. L. M. Hermens, A quantitative property-property relationship (QPPR) approach to estimate in vitro tissue-blood partition coefficients of organic chemicals in rats and humans. Archives of Toxicology, 1997. 72(1): p. 17-25.

41. Black, K. A. and L. Finch, Acrylic acid oxidation and tissue-to-blood partition coefficients in rat tissues. Toxicology Letters, 1995. 78(1): p. 73-78.

42. van Zanden, J. J., et al., Quantitative structure activity relationship studies on the flavonoid mediated inhibition of multidrug resistance proteins 1 and 2. Biochemical Pharmacology, 2005. 69(4): p. 699-708.

43. Reddy, M. B., et al., Physiological modeling of inhalation kinetics of octamethylcyclotetrasiloxane in humans during rest and exercise. Toxicological Sciences, 2003. 72(1): p. 3-18.

44. Evans, M. V. and M. E. Andersen, Sensitivity analysis of a physiological model for 2,3,7,8Tetrachlorodibenzo-p-dioxin (TCDD): Assessing the impact of specific model parameters on sequestration in liver and fat in the rat. Toxicological Sciences, 2000. 54(1): p. 71-80.

45. Olthof, M. R., et al., Bioavailabilities of quercetin-3-glucoside and quercetin-4'-glucoside do not differ in humans. The Journal of Nutrition, 2000. 130(5): p. 1200-1203.

46. Matsukawa, N., M. Matsumoto, and H. Hara, High Biliary Excretion Levels of Quercetin Metabolites after Administration of a Quercetin Glycoside in Conscious Bile Duct Cannulated Rats. Bioscience, Biotechnology, and Biochemistry, 2009. 73(8): p. 1863-1865.

47. Takenaga, N., et al., Structure-Activity Relationship inO-Glucuronidation of Indolocarbazole Analogs. Drug Metabolism and Disposition, 2002. 30(5): p. 494-497.

48. Manevski, N., et al., Glucuronidation of Psilocin and 4-Hydroxyindole by the Human UDPGlucuronosyltransferases. Drug Metabolism and Disposition, 2010. 38(3): p. 386-395.

49. Galindo, P., et al., Glucuronidated quercetin lowers blood pressure in spontaneously hypertensive rats via deconjugation. PLoS ONE, 2012. 7(3): p. e32673.

50. Bartholomé, R., et al., Deconjugation kinetics of glucuronidated phase II flavonoid metabolites by $\beta$-glucuronidase from neutrophils. Drug Metabolism and Pharmacokinetics, 2010. 25(4): p. 379-387. 
Chapter 2 


\section{CHAPTER 3}

Use of physiologically based kinetic (PBK) modeling to study interindividual human variation and species differences in plasma concentrations of quercetin and its metabolites

Rungnapa Boonpawa, Nooshin Moradi, Albertus Spenkelink, Ivonne M. C. M. Rietjens, Ans Punt

Published in:

Biochemical Pharmacology 2015; 98(4): 690-702. 


\begin{abstract}
Biological activities of flavonoids in vivo ultimately depend on the systemic bioavailability of the aglycones and their metabolites. We aimed to develop physiologically based kinetic (PBK) models to predict plasma concentrations of the flavonoid quercetin and its metabolites in individual human subjects and to define species differences compared with male rat. The human models were developed based on in vitro metabolic parameters derived from incubations with pooled and 20 individual human tissue fractions and by fitting kinetic parameters to available in vivo data. The outcomes obtained were compared to a previously developed model for quercetin and its metabolites formation in male rat. Quercetin-3'-Oglucuronide was predicted to be the major circulating metabolite in 19 out of 20 individuals, while in male rat di- and tri-conjugates of quercetin containing a glucuronic acid, sulfate and/or methyl moiety are the major metabolites. Significant species differences occur in major circulating metabolites of quercetin suggesting that rat is not an adequate model to study effects of quercetin in man. The defined PBK models can be used to guide the experimental design of in vitro experiments with flavonoids, especially to better take into account the relevance of metabolism and the contribution of metabolites to the biological activity in humans.
\end{abstract}


Inter- and intraspecies differences in metabolic plasma profiles of quercetin

\subsection{Introduction}

Flavonoids are polyphenolic compounds widely distributed in fruits and vegetables. These compounds have received a lot of attention for their possible beneficial health effects against various degenerative disorders such as cancer [1], cardiovascular diseases [2, 3], and obesity and diabetes [4]. A vast amount of in vitro studies have been conducted to elucidate modes of actions underlying these health effects. However, where most of these in vitro studies are performed with the aglycone, this form is hardly available in vivo due to extensive metabolism of the flavonoid aglycone in the intestine and liver. As a result, mainly flavonoid conjugates occur in the systemic circulation. Little is known about the nature and physiologically relevant concentrations of these circulating conjugates, nor about species differences and interindividual human variation herein. However, interpretation as well as design of both in vitro and in vivo animal and human experiments would benefit from insight in the nature and physiologically relevant concentrations of circulating conjugates. The aim of the present study was to use physiologically based kinetic (PBK) modeling to predict the nature and regiospecificity of the major circulating metabolites using quercetin as a model compound.

Quercetin is one of the most prevalent flavonoids in the human diet (e.g. onions) and also one of the most widely studied flavonoids [5]. Upon absorption, quercetin aglycone can be directly taken up in the small intestine by passive diffusion, but its glucoside needs to be deglycosylated to the aglycone by brush-border lactase phlorizin hydrolase or by intestinal microflora prior to absorption $[6,7]$. In the small intestine and liver, quercetin is metabolized via glucuronidation, sulfation and methylation (Figure 3.1). Numerous mono-, di- and triconjugates of quercetin containing glucuronic acid, sulfate and/or methyl moieties have been detected in plasma of rat as well as in human [8-10]. Various metabolites have been reported as major circulating metabolites of quercetin in humans. Mullen et al. reported quercetin-3'$\mathrm{O}$-sulfate and quercetin-3-O-glucuronide to be among the major circulating metabolites $0.5 \mathrm{~h}$ after consumption of $270 \mathrm{~g}$ fried onions [10]. Lee et al. found that quercetin monoglucuronides are major circulating metabolites $0.5 \mathrm{~h}$ after consumption of $47.5 \mathrm{~g}$ onion powder [9]. Another study by Day et al. reported that the major circulating metabolites of quercetin in plasma $1.5 \mathrm{~h}$ after consumption of $200 \mathrm{~g}$ fried onions, are quercetin-3-Oglucuronide, 3'-O-methylquercetin-3-O-glucuronide, and quercetin-3'-O-sulfate [11]. In comparison, in rats the major circulating metabolite detected $1 \mathrm{~h}$ after dosing of $7.6 \mathrm{mg} / \mathrm{kg}$ bw quercetin-4'-O-glucoside (Q4'Gly) was a tri-conjugate containing glucuronic acid, sulfate and 
Chapter 3

methyl moieties [8]. Based on our previous PBK model studies, this tri-conjugate in rat was tentatively identified as 7-O-glucuronide,-3'-O-sulfate,-4'-O-methylquercetin [12].

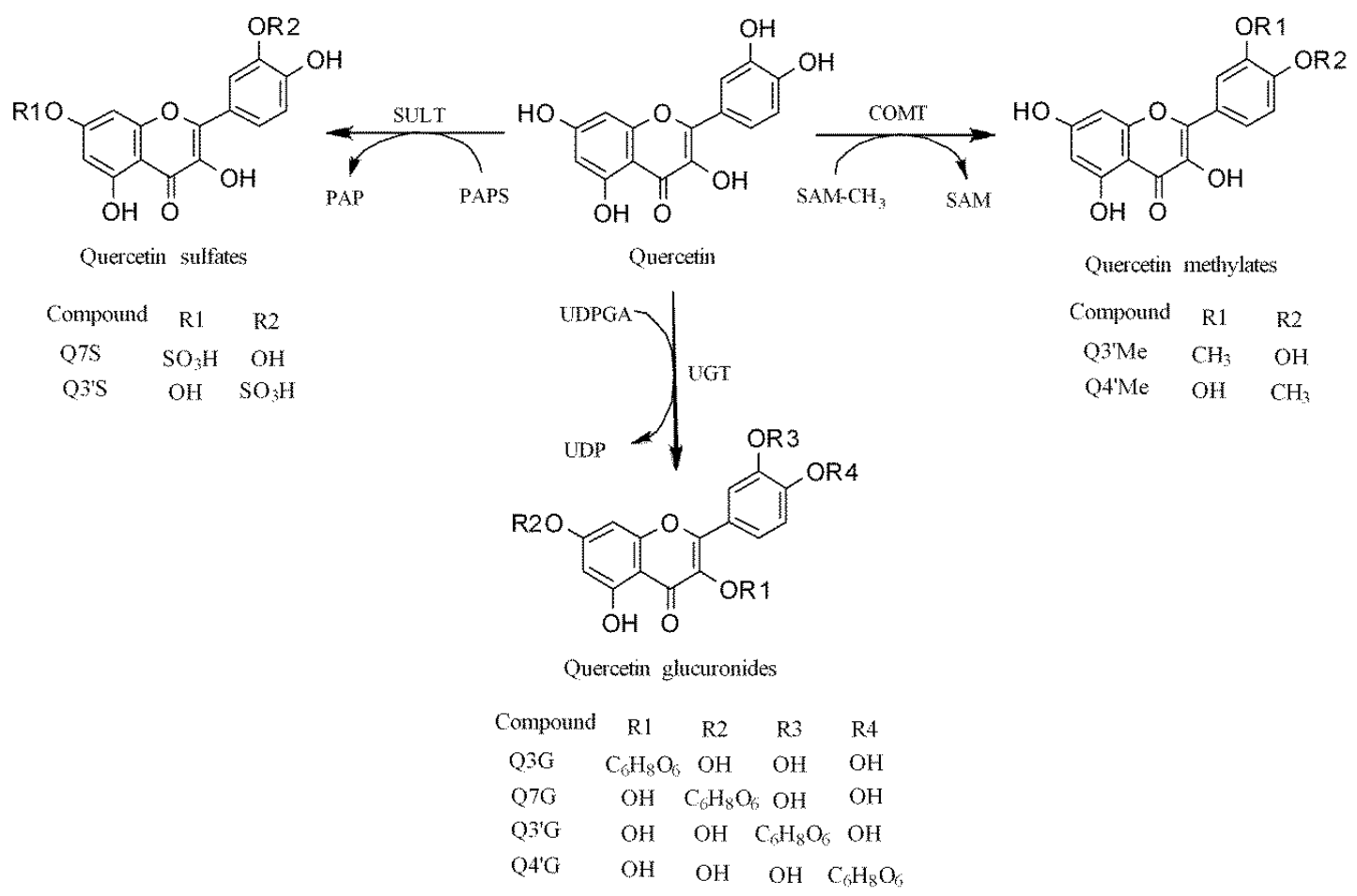

Figure 3.1 Conversion of quercetin to different possible mono-conjugates [12].

To obtain better insights in the nature and concentration of circulating metabolites of quercetin and interindividual human variation, PBK models for quercetin in (individual) humans were defined based on kinetic parameters for the formation of different metabolites obtained from in vitro incubations with relevant individual and pooled human tissue fractions and by fitting kinetic parameters to the available in vivo data [9-11]. A subsequent comparison was made with the previous defined PBK model for quercetin in male rats [12] to elucidate possible species differences in the major circulating metabolites.

\subsection{Material and methods}

\subsubsection{Materials}

Pooled, mixed-gender human small intestine and liver S9 fractions, individual human liver S9 fractions from 20 different individual, and pooled human liver cytosol were purchased from Xenotech (Lenexa, USA). Quercetin, L-ascorbic acid, uridine 5'diphosphoglucuronic acid (purity 98\%), S-(5'-adenosyl)-L-methionine (purity 80\%), 3'phosphoadenosine-5'-phosphosulfate (purity 65\%,), tris (hydroxymethyl)aminomethane, alamethicin (from Trichoderma viride), and $\beta$-glucuronidase/sulfatase (from Helix pomatia) 
Inter- and intraspecies differences in metabolic plasma profiles of quercetin

were purchased from Sigma-Aldrich (Steinheim, Germany). Potassium dihydrogen phosphate, dipotassium hydrogen phosphate trihydrate, acetic acid (glacial), hydrochloric acid (fuming 37\%), ammonium acetate, magnesium chloride, sodium acetate trihydrate, and trifluoroacetic acid (for spectroscopy) were purchased from VWR International (Darmstadt, Germany). Dimethyl sulfoxide (DMSO) was purchased from Acros Organic (New Jersey, USA). Acetonitrile (ULC/MS) and methanol (ULC/MS) were purchased from Biosolve (Valkenswaard, The Netherlands). Quercetin-3-O-glucuronide, isorhamnetin (3'-Omethylquercetin), and tamarixetin (4'-O-methylquercetin) were purchased from Extrasynthese (Genay Cedex, France).

\subsubsection{Glucuronidation of quercetin by pooled and individual human tissue fractions}

To obtain kinetic parameters for glucuronidation of quercetin, incubation mixtures (final volume of $100 \mu \mathrm{l}$ ) were prepared containing (final concentrations) $0.1 \mathrm{M}$ Tris- $\mathrm{HCl}(\mathrm{pH}$ 7.4), $10 \mathrm{mM} \mathrm{MgCl} 2,1 \mathrm{mM}$ ascorbic acid, $0.04 \mathrm{mg} / \mathrm{ml}$ pooled human small intestine or 0.03 $\mathrm{mg} / \mathrm{ml}$ pooled liver S9 fractions, and $4 \mathrm{mM}$ uridine 5'-diphosphoglucuronic acid as cofactor. The incubation mixtures were pre-treated on ice with $0.025 \mathrm{mg} / \mathrm{ml}$ alamethicin added from a 200 times concentrated stock solution in methanol for $15 \mathrm{~min}$ to obtain optimal glucuronidation activity [13]. The reactions were initiated by adding quercetin (final concentrations ranging from $0.15 \mu \mathrm{M}$ to $20 \mu \mathrm{M}$ ) added from 200 times concentrated stock solutions in DMSO after preincubating at $37^{\circ} \mathrm{C}$ for $1 \mathrm{~min}$. The reactions were terminated by adding $25 \mu \mathrm{l}$ ice-cold acetonitrile:acetic acid (94:6) (v/v) after $6 \mathrm{~min}$ or $7 \mathrm{~min}$ for incubations with small intestine S9 or liver S9. To evaluate interindividual human variation in glucuronidation, incubations with individual human liver S9 fractions were performed as described for pooled liver S9. These reactions were carried out for 5 to $20 \mathrm{~min}$ at $37^{\circ} \mathrm{C}$ and at an $\mathrm{S} 9$ protein content ranging from 0.01 to $0.03 \mathrm{mg} / \mathrm{ml}$, depending on the human subject. Under the specified conditions, the formation of quercetin monoglucuronides was linear in time and with protein concentration (data not shown). The nature of the glucuronide metabolites was confirmed by treating samples with $\beta$-glucuronidase ( $\beta$ glucuronidase/sulfatase). For these reactions, $50 \mu \mathrm{l}$ of the non-terminated mixtures were added to $50 \mu \mathrm{l}$ of $0.1 \mathrm{M}$ sodium acetate $(\mathrm{pH} 5.0)$ containing $1 \mathrm{mM}$ ascorbic acid and 0.8 units $/ \mathrm{ml} \beta$-glucuronidase. These incubations were carried for $1 \mathrm{~h}$ at $37^{\circ} \mathrm{C}$ and terminated by addition of $25 \mu 1$ ice-cold acetonitrile:acetic acid (94:6) (v/v). 


\subsubsection{Sulfation of quercetin by pooled and individual human tissue fractions}

To obtain kinetic parameters for sulfation of quercetin, incubation mixtures (final volume of $100 \mu \mathrm{l}$ ) were prepared containing (final concentrations) $0.1 \mathrm{M}$ potassium phosphate (pH 7.4), $1 \mathrm{mM}$ ascorbic acid, $0.2 \mathrm{mg} / \mathrm{ml}$ pooled human small intestine or $1 \mathrm{mg} / \mathrm{ml}$ pooled liver S9 fractions, and $0.1 \mathrm{mM}$ 3'-phosphoadenosine-5'-phosphosulfate as cofactor. Samples were preincubated at $37^{\circ} \mathrm{C}$ for 1 min prior to the addition of quercetin (final concentration ranging from $0.15 \mu \mathrm{M}$ to $4 \mu \mathrm{M}$ ) added from 200 times concentrated stock solutions in DMSO to initiate the reactions. The reactions were terminated after $12 \mathrm{~min}$ by the addition of $25 \mu 1$ ice-cold acetonitrile:acetic acid (94:6) (v/v). To evaluate interindividual human variation in sulfation, incubations with individual human liver S9 fractions were performed as described for pooled liver S9. These reactions were carried out at an S9 protein content ranging from 0.2 to $0.8 \mathrm{mg} / \mathrm{ml}$ for 6 to $40 \mathrm{~min}$, depending on the human subject. Under the specified conditions, the formation of quercetin sulfate conjugates was linear in time and with protein concentration (data not shown). Formation of the quercetin sulfate conjugates was confirmed by adding $50 \mu \mathrm{l}$ of a non-terminated mixture to $50 \mu \mathrm{l}$ of $0.2 \mathrm{M}$ potassium phosphate ( $\mathrm{pH}$ 6.2) containing $1 \mathrm{mM}$ ascorbic acid and 100 units/ml sulfatase ( $\beta$-glucuronidase/sulfatase). These samples were incubated for $1 \mathrm{~h}$ at $37^{\circ} \mathrm{C}$ and the reactions were terminated by addition of $25 \mu \mathrm{l}$ ice-cold acetonitrile:acetic acid (94:6) (v/v). To determine whether the microsomal protein in S9, which can serve as a potential source of sulfatase enzymes could influence the rate of formation of the different sulfate conjugates, incubations were performed with cytosol [14, 15]. These reactions revealed proportionally the same kinetic constants as with S9 indicating no influence of microsomes on the reaction (data not shown).

\subsubsection{Methylation of quercetin by pooled and individual human tissue fractions}

To obtain kinetic parameters for methylation of quercetin, incubation mixtures (final volume $100 \mu \mathrm{l}$ ) were prepared containing (final concentrations) $0.1 \mathrm{M}$ potassium phosphate (pH 7.4), $1 \mathrm{mM}$ ascorbic acid, $1 \mathrm{mg} / \mathrm{ml}$ pooled human small intestine or $1 \mathrm{mg} / \mathrm{ml}$ pooled liver S9 fractions, and $2 \mathrm{mM} \mathrm{S-(5'-adenosyl)-L-methionine} \mathrm{as} \mathrm{cofactor.} \mathrm{The} \mathrm{reactions} \mathrm{were}$ initiated by the addition of quercetin (final concentrations ranging from $0.25 \mu \mathrm{M}$ to $40 \mu \mathrm{M}$ ) added from a 200 times concentrated stock solution in DMSO after 1 min preincubation at $37^{\circ} \mathrm{C}$. Upon incubating for $22 \mathrm{~min}, 25 \mu \mathrm{l}$ ice-cold acetonitrile:acetic acid (94:6) (v/v) were added to terminate the reactions. To evaluate interindividual human variation in quercetin 
Inter- and intraspecies differences in metabolic plasma profiles of quercetin

methylation, incubations with individual human liver S9 fractions were performed as described for pooled liver S9. These reactions were carried out at an S9 protein content ranging from 0.3 to $1 \mathrm{mg} / \mathrm{ml}$ for 15 to $122 \mathrm{~min}$ at $37^{\circ} \mathrm{C}$, depending on the human subject. Under the specified conditions, methylation of quercetin was linear in time and with protein concentration (data not shown).

\subsubsection{UPLC analysis}

Kinetic constants were obtained from triplicate incubations for pooled tissue fractions and duplicate incubations for individual human liver fractions. Blank incubations were carried out without the cofactor. The amount of glucuronide, sulfate and methyl conjugates formed, was analyzed on a UPLC-DAD system consisting of a Waters (Milford, MA) Acquity binary solvent manager, sample manager, and photodiode array detector, equipped with a Waters Acquity UPLC BEH RP 18 column $(1.7 \mu \mathrm{m}, 2.1$ x $50 \mathrm{~mm})$. Before analysis, all samples were centrifuged at $18,600 \times \mathrm{g}$ for $5 \mathrm{~min}$ at $5^{\circ} \mathrm{C}$ to precipitate proteins and $10 \mu \mathrm{l}$ of the supernatant was immediately analyzed. For glucuronide conjugates, a gradient of $2.5 \mathrm{mM}$ ammonium acetate in nanopure water (eluent A) and acetonitrile (eluent B), was used with a flow rate of $0.6 \mathrm{ml} / \mathrm{min}$ with the following profile: $10-25 \% \mathrm{~B}$ (0-1.8 $\mathrm{min}) ; 25-90 \% \mathrm{~B}(1.8-2.5 \mathrm{~min})$; $90 \% \mathrm{~B}$ (2.5-3.8 $\mathrm{min})$; $90-10 \% \mathrm{~B}(3.8-4 \mathrm{~min})$ and $10 \% \mathrm{~B}$ (4-5 min). For sulfate and methyl conjugates, a gradient of nanopure water containing $0.1 \%(\mathrm{v} / \mathrm{v})$ trifluoroacetic acid (eluent $\mathrm{A})$ and acetonitrile (eluent B) was used with a flow rate of $0.6 \mathrm{ml} / \mathrm{min}$ with the following profile: 20-25\% B (0-1.8 min); 25-35\% B (1.8-2.3 min); 35\% B (2.3-2.8 min); 35-100\% B (2.8-3 $\min ) ; 100 \%$ B (3-4.1 min); 100-0\% B (4.1-4.3 min); 0\% B (4.3-5.5 min); 0-20\% B (5.5-5.6 $\min )$ and $20 \% \mathrm{~B}(5.6-5.8 \mathrm{~min})$.

The metabolites obtained from glucuronidation and sulfation were identified by comparing the UV-spectra and the elution order of each metabolite with those reported in the studies of Boersma et al. [16] and van der Woude et al. [17] as described previously for incubations with rat tissue fractions [12]. The metabolites obtained from incubations measuring methylation were identified by comparing the UV-spectra and the retention time with commercially available standards of $3^{\prime}$-O-methylquercetin and 4'-O-methylquercetin. All quercetin metabolites were quantified by integrating the peak areas at $370 \mathrm{~nm}$ and by using the calibration curves of quercetin-3-O-glucuronide (for glucuronide conjugates), quercetin (for sulfate conjugates), and 3'-O-methylquercetin and 4'-O-methylquercetin (for methyl conjugates). 


\subsubsection{LC-MS analysis}

The nature of the formed metabolites from glucuronidation, sulfation and methylation of quercetin was identified using LC-MS on a Perkin Elmer 200 series HPLC system (Perkin Elmer, Waltham, MA) coupled to an API 3000 system (Applied Biosystem, Foster city, CA). Aliquots of $10 \mu \mathrm{l}$ were injected on an Agilent Zorbax Extend-C18 column, 2.1 x $50 \mathrm{~mm}, 3.5$ Micron $80 \AA$ with a Zorbax guard column (Basel, Switzerland). The same gradient compositions as described in Section 3.2.5 were used, except that eluent A was nanopure water containing $0.1 \%(\mathrm{v} / \mathrm{v})$ acetic acid and a flow rate of $0.2 \mathrm{ml} / \mathrm{min}$ was used with the following profile: $20 \% \mathrm{~B}$ (0-1 $\mathrm{min}), 20-35 \% \mathrm{~B}$ (1-4 min), 35\% B (4-6 min), 35-80\% B (6-10 $\min ), 80 \% \mathrm{~B}(10-15 \mathrm{~min}), 80-20 \% \mathrm{~B}$ (15-16 $\mathrm{min})$, and 20\% B (16-20 $\mathrm{min})$. The mass spectrometric analysis was performed in the negative ion mode using a spray voltage of 4.5 $\mathrm{kV}$ and a capillary temperature of $180^{\circ} \mathrm{C}$. The nebulizer gas (air), curtain (nitrogen), declustering potential, focusing potential, and entrance potential were set to $10,8,-51,-200$, and -13.7 , respectively. Analysis was carried out using product ion $\left(\mathrm{MS}^{2}\right)$ scanning from $\mathrm{m} / \mathrm{z}$ 200-600.

\subsubsection{Kinetic analysis}

Kinetic parameters (i.e. apparent maximum velocity $\left(V_{\max (a p p)}\right)$ and apparent Michaelis-Menten constant $\left.\left(K_{\mathrm{m}(\mathrm{app})}\right)\right)$ for glucuronidation, sulfation, and methylation of quercetin were determined by fitting the data to the standard Michaelis-Menten equation:

$$
v=V_{\max } \times[\mathrm{S}] /\left(K_{\mathrm{m}}+[\mathrm{S}]\right)
$$

where $[\mathrm{S}]$ represents the substrate concentration. The data were fitted to the MichaelisMenten equation with GraphPad Prism, version 5.04 (GraphPad Software, San Diego, California, USA).

\subsubsection{Definition of the PBK model for (individual) human subjects}

The PBK models that describe the absorption, distribution, metabolism and excretion (ADME) of quercetin in (individual) humans were defined in a similar manner as used for developing the previously defined PBK model for quercetin in male rats [12]. The structure of the models is displayed in Figure 3.2. The kinetic data describing metabolic conversion of quercetin to its primary metabolites were obtained in the present study based on the in vitro experiments (see section 3.3.1-3.3.3). Even though quercetin is one of the most studied flavonoids, there are only few studies identifying and quantifying the plasma profiles of quercetin in humans [9-11]. Therefore, we only used these studies for the development of the 
models. The parameters describing excretion via urine, bile and intestinal enterocyte efflux back to lumen were fitted to the available experimental human data [9-11]. These data are primary available for onion intake, containing mainly Q4'Gly and quercetin-3,4'-Odiglucoside (Q34'diGly) [9, 10] and are hydrolyzed to the aglycone by luminal lactase phlorizin hydrolase $[18,19]$. Thus disappearance of the glucosides to the aglycone in the intestinal lumen was included and fitted to the experimental data, assuming each glucoside has its own deglycosylation rate with values ranging from $0.51-1 \mathrm{l} / \mathrm{h}$ and $0.01-0.1 \mathrm{l} / \mathrm{h}$ for Q4'Gly and Q34'diGly, respectively. The uptake rate constant of quercetin aglycone to the small intestine in humans was assumed to be similar to rats with a value of $5.32 \mathrm{~h}^{-1}$ [20]. For the PBK model describing the ADME in an average human, the kinetic constants obtained from incubations with pooled human tissue fractions were used. Whereas for the PBK models in individual human subjects, the kinetic constants obtained with the individual human liver fractions were used. No interindividual human variation in metabolism by the small intestine was taken into account in the individual human models as the plasma concentrations of quercetin metabolites is mainly influenced by the kinetic constants for formation of primary metabolites in liver (see section 3.3.4).
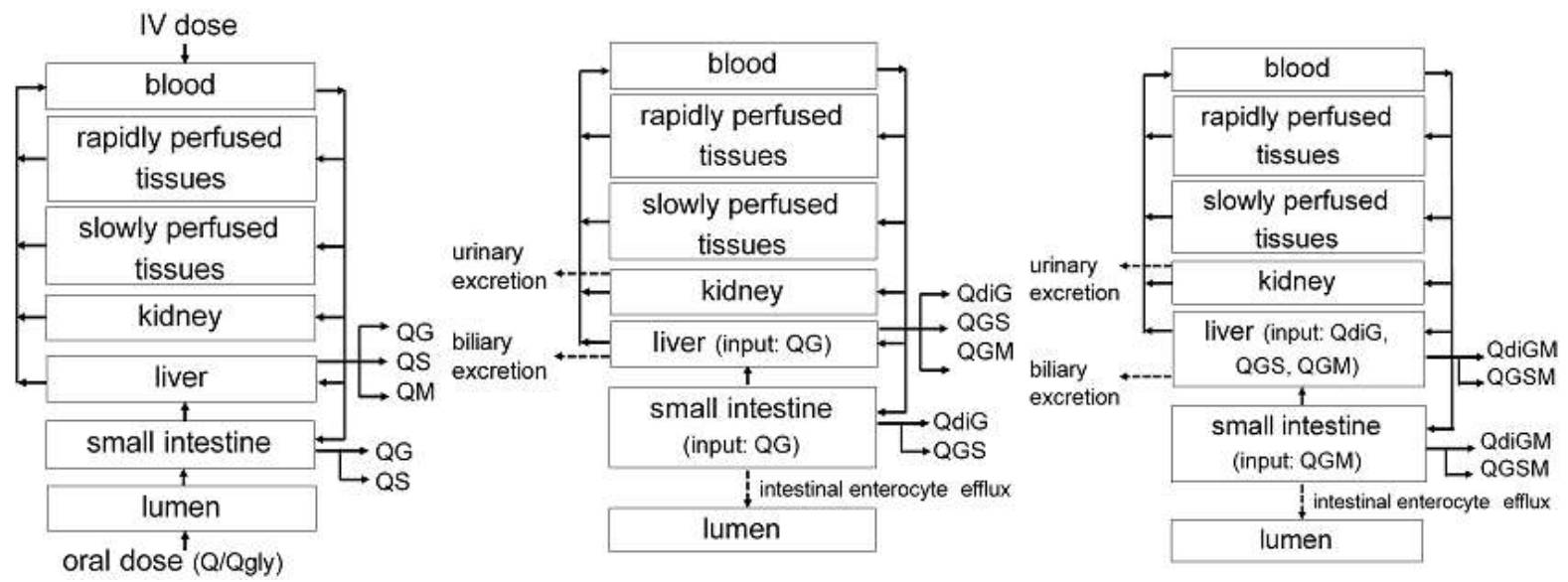

Figure 3.2 Schematic diagram of the PBK models for prediction of the formation of mono-conjugates (A), di-conjugates (B), and tri-conjugates (C).

For description of further conjugation of the primary metabolites to di- and triconjugates, only further conjugation of different monoglucuronides was simulated since the glucuronide metabolites were estimated to account for $\sim 96 \%$ of the dose (see section 3.3.4). Lower catalytic efficiencies for further glucuronidation, sulfation, and methylation of the monoglucuronides to di- and tri-conjugates were assumed in comparison to the catalytic efficiencies for formation of the respective mono-conjugates, due to both an increase in $K_{\mathrm{m}}$ and a decrease in $V_{\max }$ [12]. The correction factors for further conjugation were obtained by 


\section{Chapter 3}

fitting the predicted plasma AUC or concentrations to the available experimental data [9-11]. Taking the regiospecificity of possible conjugations into account, a total of 34 different mono-, di- and tri-conjugates could in theory be expected and were thus included in the model.

Table 3.1 Physiological parameters used in the PBK model for quercetin in human and male rat [21]

\begin{tabular}{llll}
\hline Parameters & Symbol & Human & Male rat \\
\hline Body weight (kg) & BW & 70 & 0.25 \\
Tissue volumes (\% body weight) & & & \\
$\quad$ lumen & VLu & 1.4 & 5 \\
$\quad$ small intestine & VI & 0.9 & 1.4 \\
$\quad$ liver & VL & 2.6 & 3.4 \\
kidney & VK & 0.4 & 0.7 \\
rapidly perfused tissues & VR & 4 & 3.2 \\
$\quad$ slowly perfused tissues & VS & 75.7 & 74.6 \\
blood & VB & 7.9 & 7.4 \\
Cardiac output (1/h) & QC & 310.4 & 5.4 \\
Blood flow to tissue (\% cardiac output) & & & \\
small intestine & QI & 18.1 & 15.1 \\
liver (exclude portal vein) & QL & 4.6 & 9.9 \\
$\quad$ kidney & QK & 17.5 & 14.1 \\
rapidly perfused tissues & QR & 29.8 & 36.9 \\
slowly perfused tissues & QS & 30 & 24 \\
\hline
\end{tabular}

The physiological parameters were obtained from the literature [21] and the tissue:blood partition coefficients were calculated based on the methods described by DeJongh et al. [22]. The physiological parameters and the tissue:blood partition coefficients, thus obtained and used in the models are summarised in Table 3.1 and 3.2. Mass balance equations were numerically integrated in Berkeley Madonna 8.3.18 (Macey and Oster, UC Berkeley, CA) using the Rosenbrock's algorithm for stiff system. Mass balance equations for quercetin and its metabolites in tissues and blood were similar to those previously described in the PBK model for quercetin in male rats [12]. Model performance was assessed by comparing the predicted excretions via urine, bile and intestinal enterocyte efflux back to lumen against available in vivo data $[10,23,24]$. With the obtained model, the systemic bioavailability of quercetin aglycone and the nature and regiospecificity of the major circulating quercetin metabolites in plasma were predicted. Conversion of the blood concentrations of the different metabolites to plasma concentrations was done by dividing the blood concentrations by a blood/plasma ratio (R). For quercetin and its metabolites a $\mathrm{R}$ value of 0.66 was applied and calculated using Simcyp prediction tools [25, 26]. The systemic 
Inter- and intraspecies differences in metabolic plasma profiles of quercetin

bioavailability of quercetin aglycone and the sensitivity analysis for the plasma $\mathrm{AUC}_{0-24 \mathrm{~h}}$ of quercetin metabolites were determined follow the methods previously described by Boonpawa et al. [12].

Table 3.2 Physico-chemical parameters of quercetin and its metabolites in humans

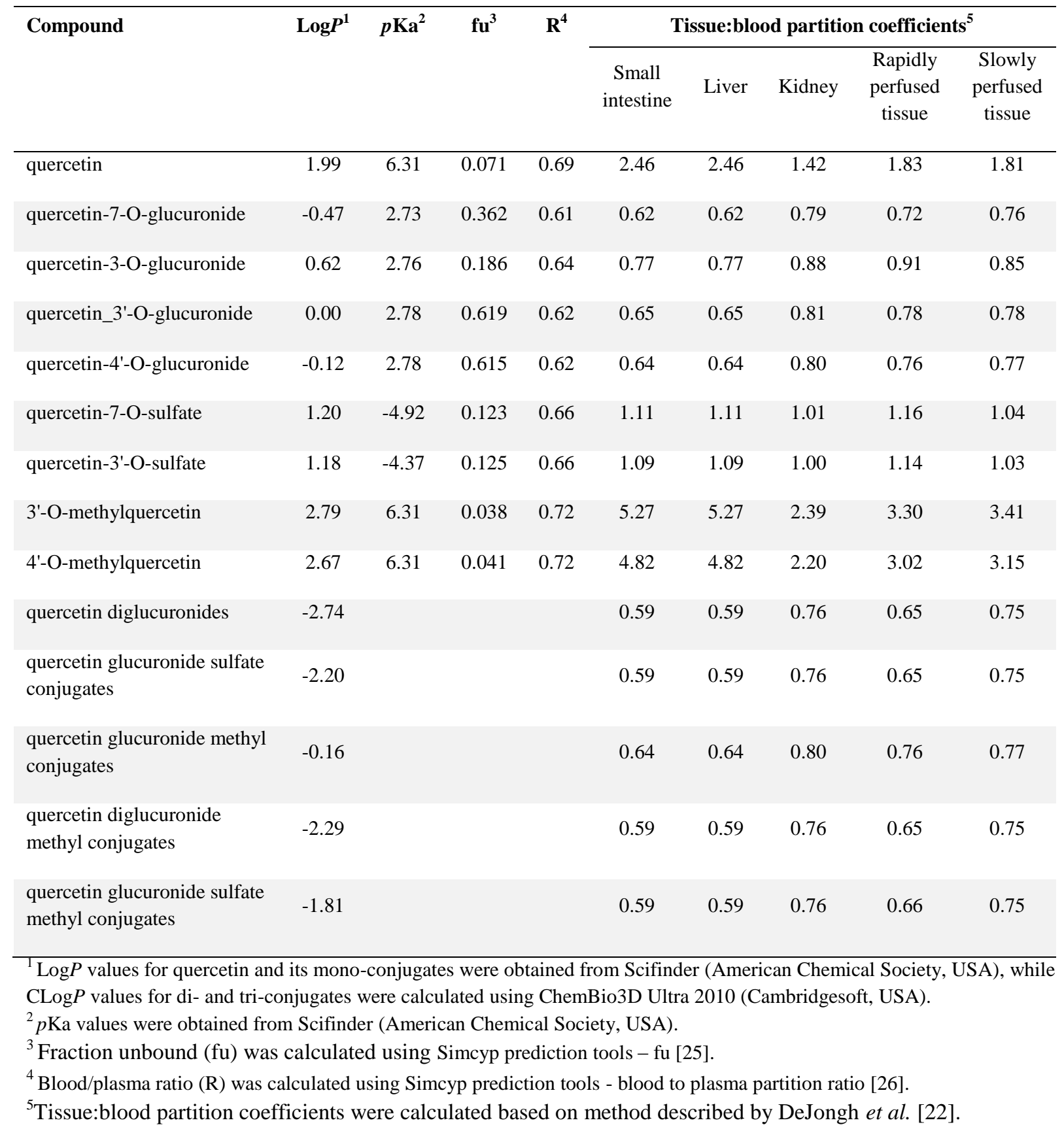

\subsection{Results}

3.3.1 Glucuronidation of quercetin by pooled and individual human tissue samples Analysis of the incubations with pooled human liver and intestine S9 revealed that both fractions were able to metabolize quercetin towards different types of glucuronide 
Chapter 3

conjugates. In incubations with human small intestine S9, quercetin-7-O-glucuronide, quercetin-3-O-glucuronide, quercetin-3'-O-glucuronide, and quercetin-4'-O-glucuronide were formed. The same metabolites, except for quercetin-4'-O-glucuronide, were formed in incubations with human liver S9. The LC-MS results confirmed that the formed metabolites were quercetin monoglucuronide as all formed metabolites had an $\mathbf{M}^{-}$at $m / z$ 477, which fragmented to produce $\mathrm{m} / \mathrm{z} 301$ (quercetin). The quercetin concentration dependent rate of formation of the monoglucuronides in these incubations with pooled liver or small intestine S9 is shown in Figure 3.3A and 3.3B. For both tissues, quercetin-3'-O-glucuronide was formed with the highest catalytic efficiency, which was 5- to 18-fold higher than the catalytic efficiency for formation of the other glucuronide conjugates in these tissues (Table 3.3).
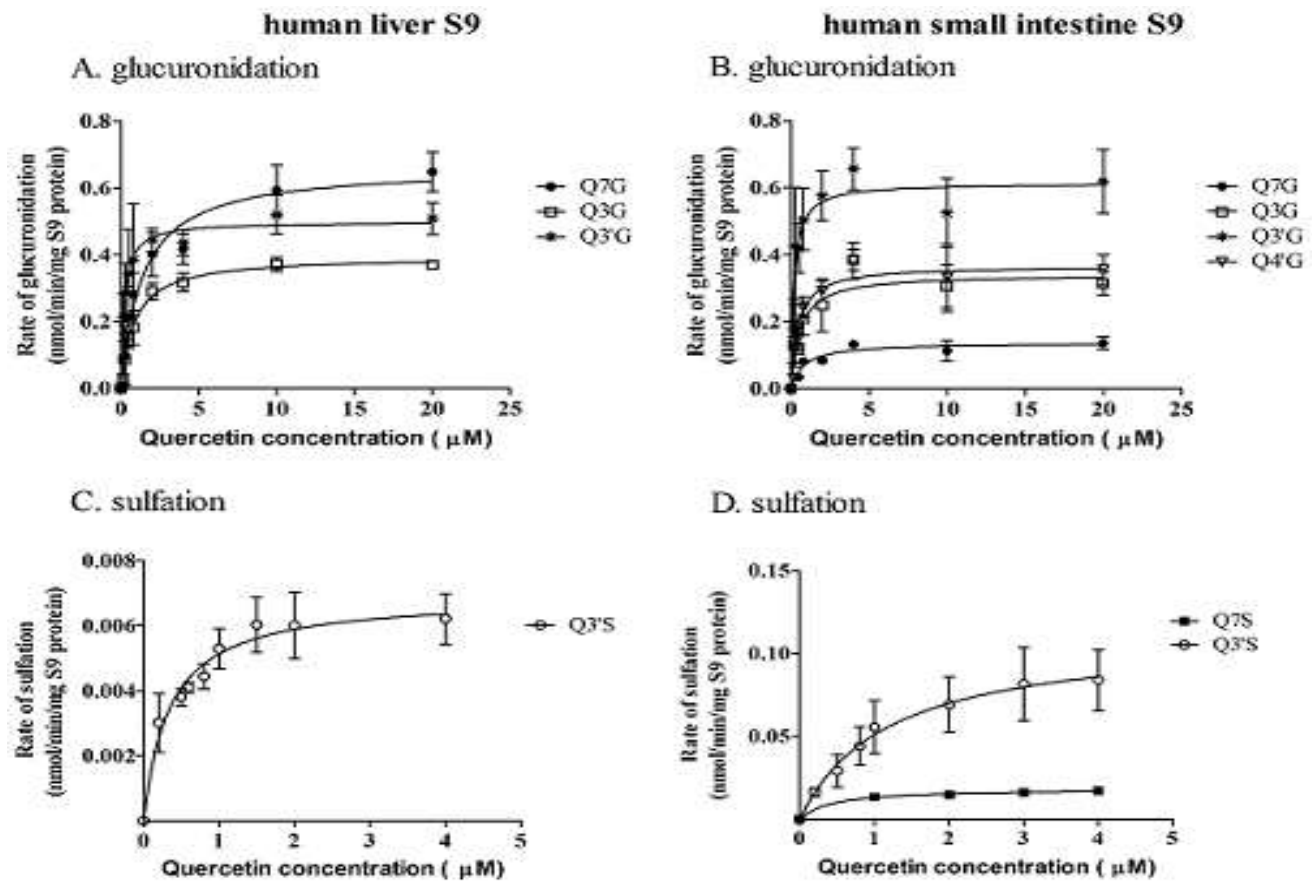

D. sulfation

E. methylation
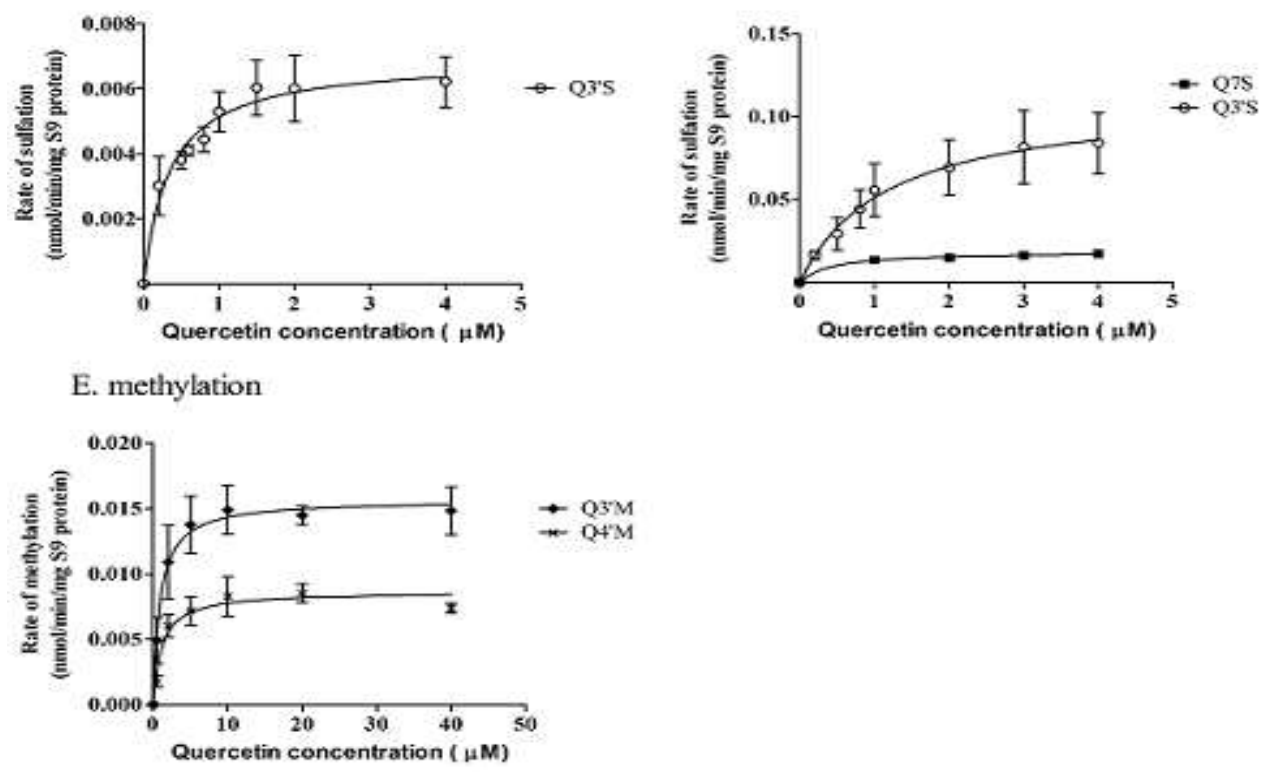

Figure 3.3 Quercetin concentration-dependent formation of mono-conjugates by pooled mixed-gender human liver S9 (A, C, and E) or small intestine S9 (B and D); quercetin (Q), glucuronide (G), sulfate (S) and methyl conjugate (M).

Analysis of interindividual human variation in formation of monoglucuronides revealed 39-fold variation in the catalytic efficiencies for formation of these metabolites between the 20 individual human liver S9 samples that were analyzed (Table 3.4). Quercetin- 
7-O-glucuronide and quercetin-3'-O-glucuronide were formed with the highest catalytic efficiency by individual H0420 (male, Caucasian, 42 years) and quercetin-3-O-glucuronide by individual H0270 (male, Caucasian, 5 months). All three conjugates were formed with the lowest catalytic efficiency by individual H0291 (female, Caucasian, 18 years). The latter differences were due to both a \pm 3 -fold lower $V_{\max }$ and $\mathrm{a} \pm 20$-fold higher $K_{\mathrm{m}}$ for glucuronidation by individual H0291. The incubations with individual human liver S9 fractions also revealed that quercetin-3'-O-glucuronide is the predominant glucuronide formed by 18 out of 20 individual human liver S9.

Table 3.3 Kinetic constants for mono-conjugates of quercetin with pooled mixed-gender human tissues $\mathrm{S} 9$ fractions

\begin{tabular}{lcccc}
\hline Metabolite & Organ & $\begin{array}{c}\boldsymbol{K}_{\mathbf{m}(\mathbf{a p p})} \\
(\mu \mathrm{M})\end{array}$ & $\begin{array}{c}\boldsymbol{V}_{\mathbf{m a x}(\mathbf{a p p})} \\
(\mathrm{nmol} / \mathrm{min} / \mathrm{mg} \mathrm{S} 9)\end{array}$ & $\begin{array}{c}\text { In vitro catalytic } \\
\text { efficiency } \\
(\mathrm{min} / \mathrm{mg} \mathrm{S9})^{1}\end{array}$ \\
\hline quercetin-7-O-glucuronide & small intestine & $0.84 \pm 0.23$ & $0.14 \pm 0.01$ & 0.17 \\
quercetin-3-O-glucuronide & small intestine & $0.56 \pm 0.15$ & $0.34 \pm 0.02$ & 0.61 \\
quercetin-3'-O-glucuronide & small intestine & $0.20 \pm 0.05$ & $0.61 \pm 0.03$ & 3.05 \\
quercetin-4'-O-glucuronide & small intestine & $0.46 \pm 0.12$ & $0.37 \pm 0.02$ & 0.80 \\
quercetin-7-O-sulfate & small intestine & $0.41 \pm 0.14$ & $0.02 \pm 0.001$ & 0.05 \\
quercetin-3'-O-sulfate & small intestine & $1.17 \pm 0.35$ & $0.11 \pm 0.01$ & 0.09 \\
quercetin-7-O-glucuronide & liver & $1.37 \pm 0.04$ & $0.66 \pm 0.04$ & 0.48 \\
quercetin-3-O-glucuronide & liver & $0.87 \pm 0.14$ & $0.39 \pm 0.02$ & 0.45 \\
quercetin-3'-O-glucuronide & liver & $0.21 \pm 0.08$ & $0.40 \pm 0.02$ & 2.67 \\
quercetin-4'-O-glucuronide & liver & $\mathrm{nd}$ & $\mathrm{nd}$ & $\mathrm{nd}$ \\
quercetin-7-O-sulfate & liver & $\mathrm{nd}$ & $\mathrm{nd}$ & $\mathrm{nd}$ \\
quercetin-3'-O-sulfate & liver & $0.35 \pm 0.07$ & $0.01 \pm 0.0004$ & 0.03 \\
3'-O-methylquercetin & liver & $0.93 \pm 0.21$ & $0.02 \pm 0.001$ & 0.02 \\
4'-O-methylquercetin & liver & $1.14 \pm 0.27$ & $0.01 \pm 0.0004$ & 0.01 \\
\hline${ }^{1} V_{\text {max(app) }} K_{\mathrm{m}(\text { app) }}$ & & & &
\end{tabular}

\subsubsection{Sulfation of quercetin by pooled and individual human tissue samples}

Analysis of the incubations with pooled human liver and intestine S9 revealed that both fractions were able to metabolize quercetin to sulfate conjugates. Quercetin-3'-O-sulfate was the predominant sulfate metabolite formed in incubations with human intestine $S 9$ and quercetin-7-O-sulfate was a minor metabolite. In incubations with human liver S9 only quercetin-3'-O-sulfate was formed. The LC-MS results confirmed that the formed metabolites were quercetin sulfate as all formed metabolites had an $\mathbf{M}^{-}$at $m / z 381$, which fragmented to produce $m / z 301$ (quercetin). The quercetin concentration dependent rate of formation of the sulfate conjugates in these incubations with pooled human liver or small intestine S9 is shown 


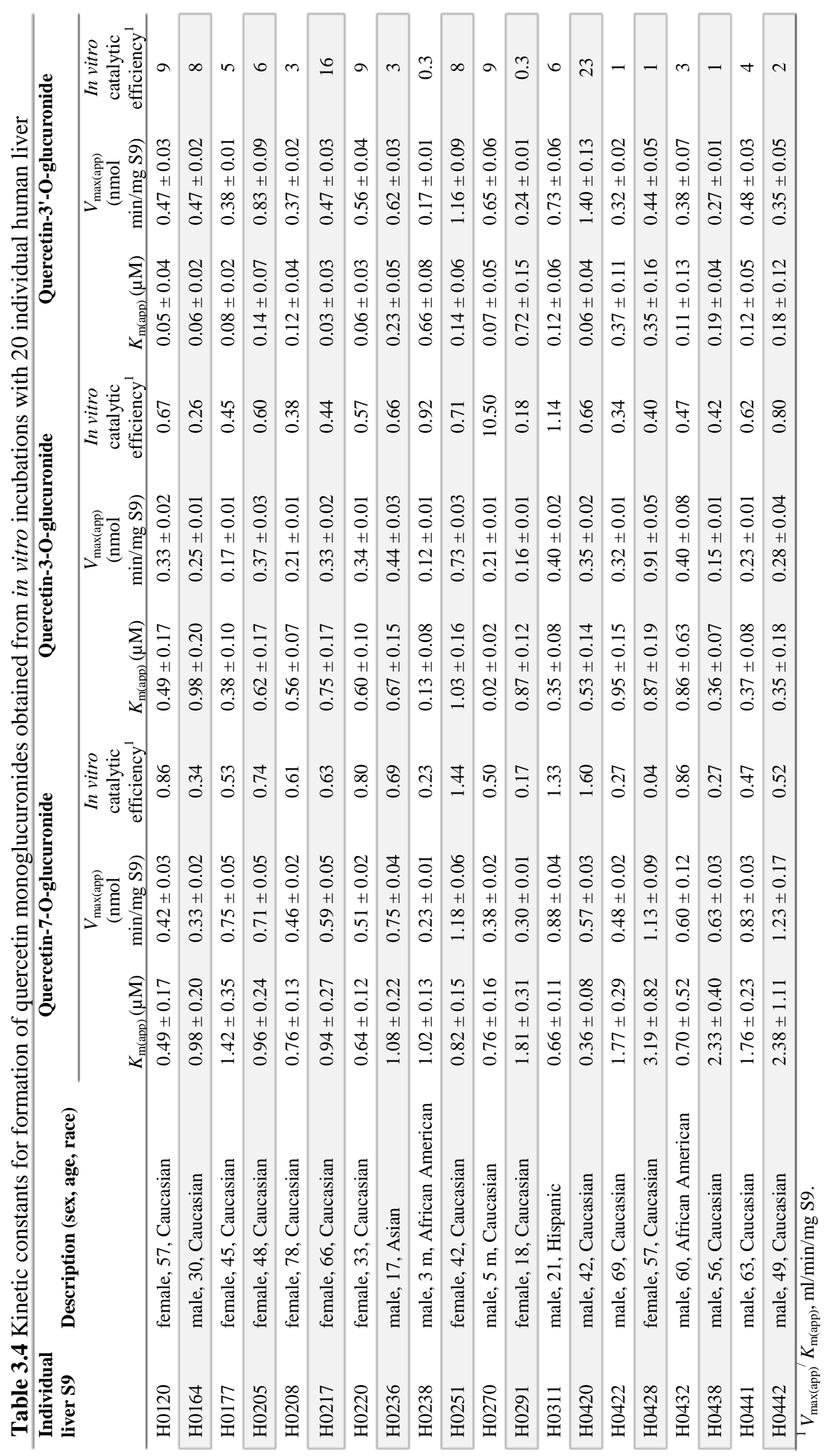


in Figure 3.3C and 3.3D and the kinetic constants derived from these curves are displayed in Table 3.3 .

In incubations with individual human liver S9 also only quercetin-3'-O-sulfate was formed, showing an 833-fold variation in formation of this metabolite between the different human samples (Table 3.5). The highest catalytic efficiency was observed for individual H0251 (female, Caucasian, 42 years), and the lowest for individual H0420 (male, Caucasian, 42 years). The differences between these individuals were due to 13- and 76-fold variation in the $V_{\max }$ and the $K_{\mathrm{m}}$ for sulfation respectively (Table 3.5).

Table 3.5 Kinetic constants for formation of quercetin monosulfates obtained from in vitro incubations with 20 individual human liver S9 fractions

\begin{tabular}{|c|c|c|c|c|}
\hline \multirow{2}{*}{$\begin{array}{l}\text { Individual } \\
\text { liver S9 }\end{array}$} & \multirow{2}{*}{ Description (sex, age, race) } & \multicolumn{3}{|c|}{ Quercetin-3'-O-sulfate } \\
\hline & & $K_{\mathrm{m}(\mathrm{app})}(\mu \mathrm{M})$ & $\begin{array}{c}V_{\max (\text { app })}(\mathrm{nmol} \\
\min / \mathrm{mg} \mathrm{S} 9)\end{array}$ & $\begin{array}{c}\text { In vitro catalytic } \\
\text { efficiency }^{1}\end{array}$ \\
\hline H0120 & female, 57, Caucasian & $0.13 \pm 0.06$ & $0.010 \pm 0.001$ & 0.08 \\
\hline H0164 & male, 30, Caucasian & $0.11 \pm 0.04$ & $0.011 \pm 0.001$ & 0.10 \\
\hline H0177 & female, 45, Caucasian & $0.13 \pm 0.06$ & $0.003 \pm 0.0003$ & 0.02 \\
\hline H0205 & female, 48, Caucasian & $0.22 \pm 0.03$ & $0.010 \pm 0.0003$ & 0.05 \\
\hline H0208 & female, 78, Caucasian & $0.02 \pm 0.02$ & $0.015 \pm 0.001$ & 0.75 \\
\hline H0217 & female, 66, Caucasian & $0.11 \pm 0.03$ & $0.008 \pm 0.0003$ & 0.07 \\
\hline $\mathrm{H} 0220$ & female, 33, Caucasian & $0.04 \pm 0.01$ & $0.013 \pm 0.0004$ & 0.33 \\
\hline H0236 & male, 17, Asian & $0.02 \pm 0.01$ & $0.005 \pm 0.0001$ & 0.25 \\
\hline $\mathrm{H} 0238$ & male, 3 m, African American & $0.15 \pm 0.07$ & $0.010 \pm 0.001$ & 0.07 \\
\hline $\mathrm{H} 0251$ & female, 42, Caucasian & $0.01 \pm 0.02$ & $0.025 \pm 0.002$ & 2.50 \\
\hline $\mathrm{H} 0270$ & male, 5 m, Caucasian & $0.04 \pm 0.03$ & $0.019 \pm 0.002$ & 0.48 \\
\hline H0291 & female, 18, Caucasian & $0.57 \pm 0.24$ & $0.009 \pm 0.001$ & 0.02 \\
\hline H0311 & male, 21, Hispanic & $0.09 \pm 0.03$ & $0.016 \pm 0.001$ & 0.18 \\
\hline H0420 & male, 42, Caucasian & $0.76 \pm 0.16$ & $0.002 \pm 0.0002$ & 0.003 \\
\hline $\mathrm{H} 0422$ & male, 69, Caucasian & $0.06 \pm 0.01$ & $0.010 \pm 0.0002$ & 0.17 \\
\hline H0428 & female, 57, Caucasian & $0.02 \pm 0.02$ & $0.021 \pm 0.001$ & 1.19 \\
\hline H0432 & male, 60, African American & $0.12 \pm 0.06$ & $0.007 \pm 0.001$ & 0.06 \\
\hline H0438 & male, 56, Caucasian & $0.06 \pm 0.04$ & $0.012 \pm 0.001$ & 0.20 \\
\hline H0441 & male, 63, Caucasian & $0.09 \pm 0.05$ & $0.005 \pm 0.0005$ & 0.06 \\
\hline H0442 & male, 49, Caucasian & $0.03 \pm 0.01$ & $0.014 \pm 0.001$ & 0.47 \\
\hline
\end{tabular}

3.3.3 Methylation of quercetin by pooled and individual human tissue samples

Analysis of the incubations with pooled human liver and intestine S9 revealed that only liver S9 was able to metabolize quercetin to methyl conjugates. In incubations with liver S9, both 3'-O-methylquercetin and 4'-O-methylquercetin were formed. The LC-MS results confirmed that the formed metabolites were quercetin methyl conjugates as all formed 
Chapter 3

metabolites had an $\mathbf{M}^{-}$at $m / z 315$, which fragmented to produce $m / z 301$ (quercetin). Figure 3.3E shows the quercetin concentration dependent rate of formation of methyl conjugates of quercetin in incubations with human liver S9 fractions and the kinetic constants derived from these curves are displayed in Table 3.3. The highest catalytic efficiency was observed in individual H0420 (male, Caucasian, 42 years) and the lowest in individual H0238 (male, African American, 3 months) (Table 3.6). These differences were mainly due to a \pm 80 -fold higher $K_{\mathrm{m}}$ for methylation by individual $\mathrm{H} 0238$.

Table 3.6 Kinetic constants for formation of quercetin methyl conjugates obtained from in vitro incubations with 20 individual human liver S9 fractions

\begin{tabular}{|c|c|c|c|c|c|c|c|}
\hline \multirow{2}{*}{$\begin{array}{l}\text { Individual } \\
\text { liver S9 }\end{array}$} & \multirow{2}{*}{$\begin{array}{l}\text { Description } \\
\text { (sex, age, } \\
\text { race) }\end{array}$} & \multicolumn{3}{|c|}{ 3'-O-methylquercetin } & \multicolumn{3}{|c|}{ 4'-O-methylquercetin } \\
\hline & & $K_{\mathrm{m}(\mathrm{app})}(\mu \mathrm{M})$ & $\begin{array}{c}V_{\max (\mathrm{app})}(\mathrm{nmol} \\
\mathrm{min} / \mathrm{mg} \mathrm{S} 9)\end{array}$ & $\begin{array}{l}\text { In vitro } \\
\mathrm{CE}^{1}\end{array}$ & $\begin{array}{c}K_{\mathrm{m}(\mathrm{app})} \\
(\mu \mathrm{M})\end{array}$ & $\begin{array}{c}V_{\max (\mathrm{app})}(\mathrm{nmol} \\
\mathrm{min} / \mathrm{mg} \mathrm{S} 9)\end{array}$ & $\begin{array}{c}\text { In vitro } \\
\mathrm{CE}^{1}\end{array}$ \\
\hline H0120 & $\begin{array}{l}\text { female, } 57, \\
\text { Caucasian }\end{array}$ & $0.65 \pm 0.29$ & $0.003 \pm 0.0003$ & 0.005 & $0.34 \pm 0.29$ & $0.001 \pm 0.0002$ & 0.003 \\
\hline H0164 & $\begin{array}{l}\text { male, } 30, \\
\text { Caucasian }\end{array}$ & $0.15 \pm 0.13$ & $0.001 \pm 0.0001$ & 0.01 & $4.46 \pm 1.64$ & $0.001 \pm 0.0001$ & 0.0002 \\
\hline H0177 & $\begin{array}{l}\text { female, } 45 \text {, } \\
\text { Caucasian }\end{array}$ & $0.86 \pm 0.19$ & $0.010 \pm 0.0005$ & 0.01 & $1.07 \pm 0.52$ & $0.003 \pm 0.0004$ & 0.003 \\
\hline H0205 & $\begin{array}{l}\text { female, } 48, \\
\text { Caucasian }\end{array}$ & $0.40 \pm 0.18$ & $0.002 \pm 0.0002$ & 0.01 & $1.27 \pm 0.85$ & $0.001 \pm 0.0002$ & 0.001 \\
\hline H0208 & $\begin{array}{l}\text { female, } 78, \\
\text { Caucasian }\end{array}$ & $0.50 \pm 0.30$ & $0.002 \pm 0.0003$ & 0.004 & $2.63 \pm 2.62$ & $0.003 \pm 0.001$ & 0.001 \\
\hline H0217 & $\begin{array}{l}\text { female, } 66, \\
\text { Caucasian }\end{array}$ & $0.22 \pm 0.23$ & $0.005 \pm 0.001$ & 0.02 & $0.53 \pm 0.24$ & $0.003 \pm 0.0002$ & 0.01 \\
\hline $\mathrm{H} 0220$ & $\begin{array}{l}\text { female, } 33 \text {, } \\
\text { Caucasian }\end{array}$ & $0.15 \pm 0.12$ & $0.002 \pm 0.0002$ & 0.01 & $9.01 \pm 6.50$ & $0.002 \pm 0.001$ & 0.0002 \\
\hline H0236 & $\begin{array}{l}\text { male, } 17, \\
\text { Asian }\end{array}$ & $0.47 \pm 0.30$ & $0.003 \pm 0.0004$ & 0.01 & $2.61 \pm 2.37$ & $0.002 \pm 0.001$ & 0.001 \\
\hline H0238 & $\begin{array}{l}\text { male, } 3 \mathrm{~m} \text {, } \\
\text { African } \\
\text { American }\end{array}$ & $60.5 \pm 61.5$ & $0.010 \pm 0.01$ & 0.0002 & $37.1 \pm 27.7$ & $0.003 \pm 0.001$ & 0.0001 \\
\hline $\mathrm{H} 0251$ & $\begin{array}{l}\text { female, } 42 \text {, } \\
\text { Caucasian }\end{array}$ & $0.57 \pm 0.52$ & $0.010 \pm 0.002$ & 0.02 & $0.78 \pm 0.38$ & $0.006 \pm 0.001$ & 0.01 \\
\hline $\mathrm{H} 0270$ & $\begin{array}{l}\text { male, } 5 \mathrm{~m} \text {, } \\
\text { Caucasian }\end{array}$ & $0.14 \pm 0.13$ & $0.001 \pm 0.0001$ & 0.01 & $0.90 \pm 0.60$ & $0.0002 \pm 0.0001$ & 0.0002 \\
\hline H0291 & $\begin{array}{l}\text { female, } 18, \\
\text { Caucasian }\end{array}$ & $0.07 \pm 0.08$ & $0.002 \pm 0.0002$ & 0.03 & $2.05 \pm 0.56$ & $0.001 \pm 0.0001$ & 0.0005 \\
\hline H0311 & $\begin{array}{l}\text { male, } 21 \text {, } \\
\text { Hispanic }\end{array}$ & $0.44 \pm 0.08$ & $0.010 \pm 0.0003$ & 0.02 & $1.00 \pm 0.25$ & $0.004 \pm 0.0002$ & 0.004 \\
\hline H0420 & $\begin{array}{l}\text { male, } 42 \text {, } \\
\text { Caucasian }\end{array}$ & $0.46 \pm 0.18$ & $0.014 \pm 0.001$ & 0.03 & $1.29 \pm 0.72$ & $0.008 \pm 0.001$ & 0.01 \\
\hline H0422 & $\begin{array}{l}\text { male, } 69, \\
\text { Caucasian }\end{array}$ & $1.17 \pm 0.48$ & $0.020 \pm 0.002$ & 0.02 & $1.92 \pm 1.00$ & $0.010 \pm 0.001$ & 0.01 \\
\hline H0428 & $\begin{array}{l}\text { female, } 57, \\
\text { Caucasian }\end{array}$ & $2.44 \pm 1.60$ & $0.022 \pm 0.004$ & 0.01 & $4.63 \pm 3.21$ & $0.015 \pm 0.004$ & 0.003 \\
\hline H0432 & $\begin{array}{l}\text { male, } 60, \\
\text { African } \\
\text { American }\end{array}$ & $1.49 \pm 0.64$ & $0.008 \pm 0.001$ & 0.01 & $3.22 \pm 0.98$ & $0.005 \pm 0.0005$ & 0.002 \\
\hline H0438 & $\begin{array}{l}\text { male, } 56, \\
\text { Caucasian }\end{array}$ & $0.50 \pm 0.19$ & $0.014 \pm 0.001$ & 0.03 & $1.39 \pm 0.72$ & $0.008 \pm 0.001$ & 0.006 \\
\hline H0441 & $\begin{array}{l}\text { male, 63, } \\
\text { Caucasian }\end{array}$ & $1.12 \pm 0.86$ & $0.007 \pm 0.001$ & 0.01 & $2.36 \pm 2.06$ & $0.003 \pm 0.001$ & 0.001 \\
\hline H0442 & $\begin{array}{l}\text { male, } 49, \\
\text { Caucasian }\end{array}$ & $0.56 \pm 0.27$ & $0.004 \pm 0.0004$ & 0.01 & $0.81 \pm 0.53$ & $0.002 \pm 0.0003$ & 0.002 \\
\hline
\end{tabular}

${ }^{1}$ in vitro catalytic efficiency was calculated by $V_{\max (\text { app })} / K_{\mathrm{m}(\mathrm{app})}$ and expressed in $\mathrm{ml} / \mathrm{min} / \mathrm{mg} \mathrm{S}$. 
3.3.4 Development of the (individual) human PBK models and evaluation of their performance

The kinetic constants obtained from in vitro incubations with pooled and individual human tissue fractions were combined into PBK models to predict the bioavailability of quercetin aglycone. The average predicted bioavailability of quercetin aglycone was $0.005 \%$ of the dose and ranged between 0.001 and $0.04 \%$ of the dose for the individual human models at an average dietary intake of $0.4 \mathrm{mg} / \mathrm{kg}$ bw quercetin. Quercetin was completely metabolized within $1.5 \mathrm{~h}$ for all individuals and $\sim 96 \%$ of the dose was converted to monoglucuronides. Among the mono-conjugates, quercetin-3'-O-glucuronide was predicted to be formed to the highest extent accounting for 53 to $57 \%$ of the dose.

A.

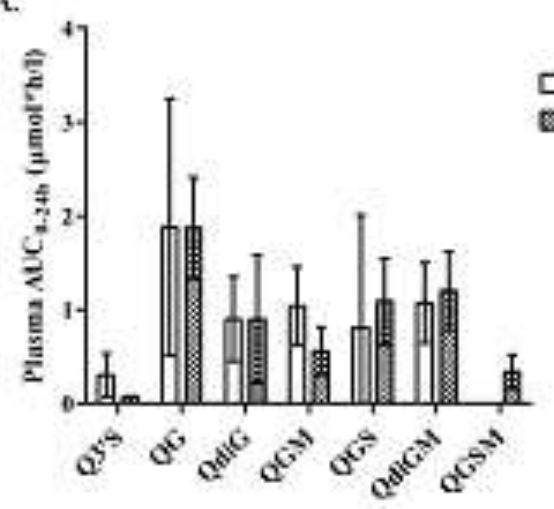

B.

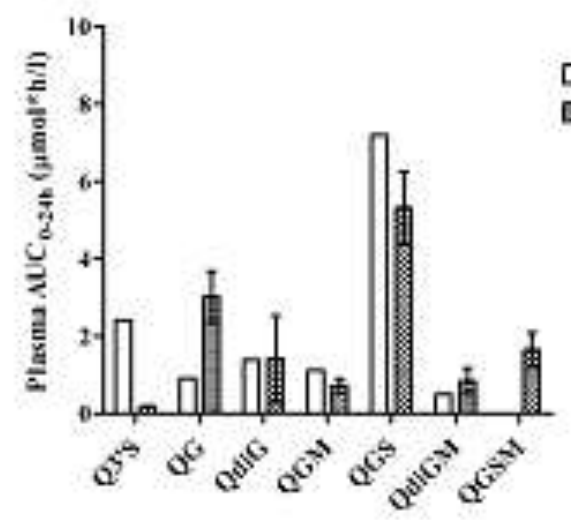

C.

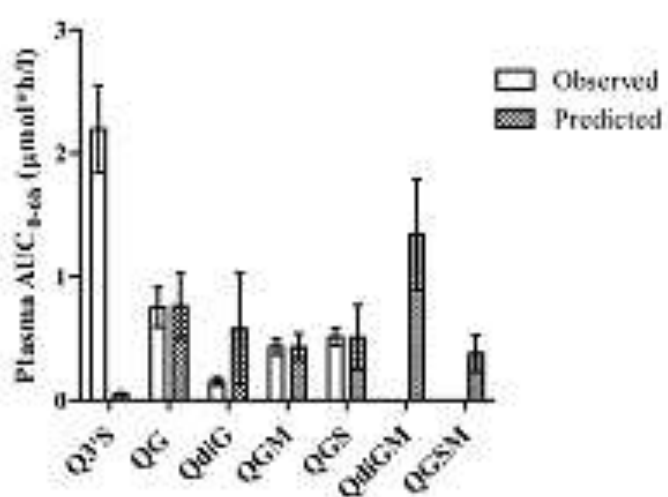

D.

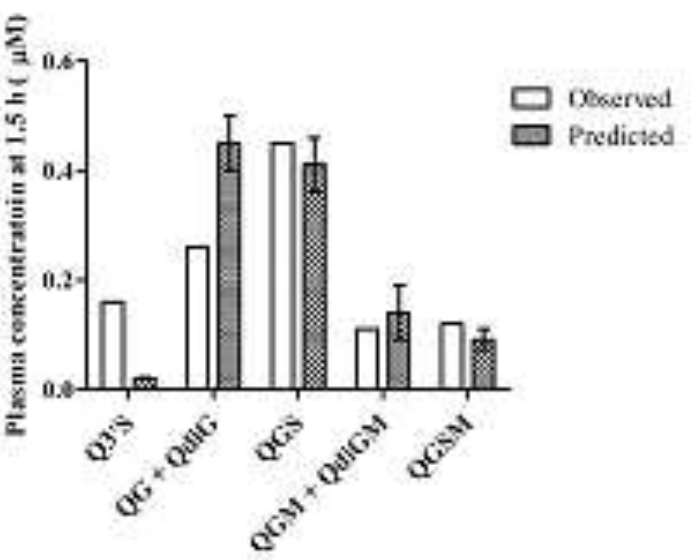

Figure 3.4 Prediction on plasma profiles of quercetin metabolites in (individual) human subjects compared to the reported levels in vivo at a dose of $0.64 \mathrm{mg} / \mathrm{kg}$ bw Q4'Gly and $0.70 \mathrm{mg} / \mathrm{kg}$ bw Q34'diGly as detected with positive (A) and negative detection mode (B) [9], at a dose of $0.95 \mathrm{mg} / \mathrm{kg}$ bw Q4'Gly and $0.96 \mathrm{mg} / \mathrm{kg}$ bw Q34'diGly (C) [10], at a dose of $0.56 \mathrm{mg} / \mathrm{kg}$ bw Q4'Gly and 0.53 $\mathrm{mg} / \mathrm{kg}$ bw Q34'diGly [11] (D); quercetin (Q), glucuronide (G), sulfate (S) and methyl conjugate (M).

Further conjugation of different monoglucuronides to di- and tri-conjugates was simulated. Because in vivo also quercetin-3'-O-sulfate has been detected in the plasma, the 


\section{Chapter 3}

formation of this metabolite was included in the comparison between the model predictions and in vivo data, but given the low overall percentage of its formation (i.e $<3 \%$ of the dose), this metabolite was not simulated to be subject to further conjugation. In general, the fitted models showed comparable plasma levels of metabolites to the levels reported in literatures (i.e. within 1- to 4-fold) (Figure 3.4). Only for quercetin-3'-O-sulfate predictions were 4- to 44-fold lower than the reported values in the three studies [9-11]. For further development of the model for prediction of di- and tri-conjugates, parameters obtained with the data from the study of Lee et al. [9] with positive detection mode were used since these provided the best fit, with a 4-fold deviation between predicted and observed values for quercetin-3'-O-sulfate (Figure 3.4A). The fitted parameters obtained are displayed in the Table 3.7.

A.

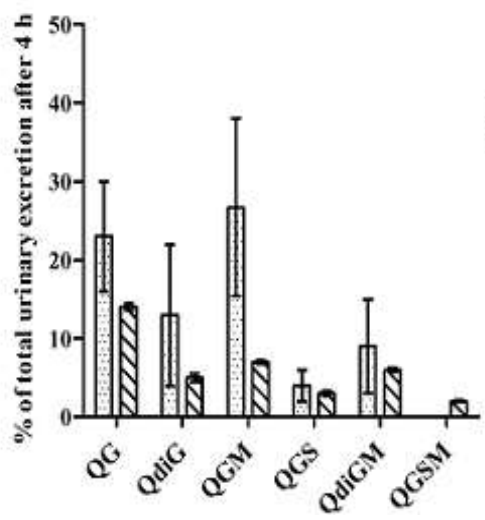

B.

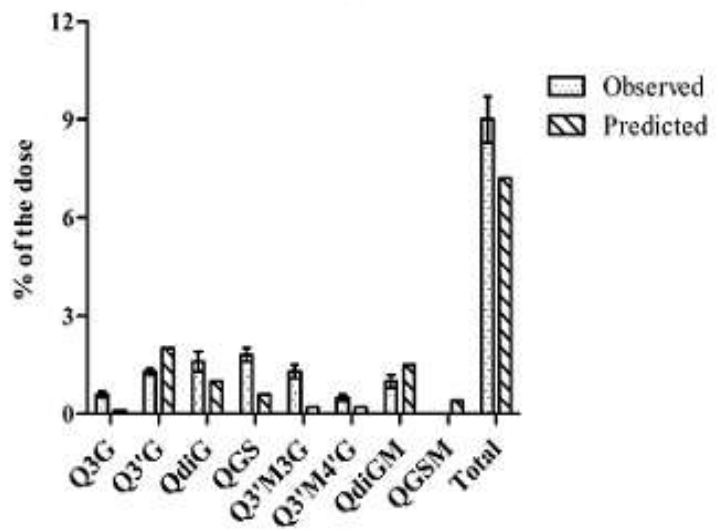

Figure 3.5 Prediction on urinary profiles of quercetin metabolites as compared with the reported levels in vivo at a dose of $0.46 \mathrm{mg} / \mathrm{kg}$ bw Q4'Gly and $0.49 \mathrm{mg} / \mathrm{kg}$ bw Q34'diGly (A) [23] and 0.95 $\mathrm{mg} / \mathrm{kg}$ bw Q4'Gly and $0.96 \mathrm{mg} / \mathrm{kg}$ bw Q34'diGly (B) [10]; quercetin (Q), glucuronide (G), sulfate (S) and methyl conjugate $(\mathrm{M})$.

With the adequate predictions of the plasma levels of the different metabolites obtained by the (individual) human PBK models, the performance of models was further assessed by comparing the predicted excretion of quercetin metabolites via urine, bile and intestinal enterocyte efflux back to lumen to the reported levels in vivo [10, 23, 24]. Figure 3.5 shows that the models adequately predicted urinary profiles with 1- to 4-fold and 1- to 7-fold accuracy $4 \mathrm{~h}$ and $24 \mathrm{~h}$ after consumption of onions corresponding to dose levels of 0.46 $\mathrm{mg} / \mathrm{kg}$ bw Q4'Gly and $0.49 \mathrm{mg} / \mathrm{kg}$ bw Q34'diGly [23] and 0.95 Q4'Gly and $0.96 \mathrm{mg} / \mathrm{kg}$ bw Q34'diGly [10]. After $72 \mathrm{~h}$ following consumption of $1.4 \mathrm{mg} / \mathrm{kg}$ bw ${ }^{14} \mathrm{C}$-quercetin, 3.5 to $5.7 \%$ of the dose was recovered in the urine which is 1 - to 2 -fold lower than the model prediction of $7.5 \%$ of the dose at the same dose [24]. In the same study $52.1 \pm 6.4 \%$ of the dose was recovered as ${ }^{14} \mathrm{CO}_{2}$ in the expired air. This value can be used to evaluate the biliary 
Inter- and intraspecies differences in metabolic plasma profiles of quercetin

excretion and intestinal efflux back to lumen, because ${ }^{14} \mathrm{CO}_{2}$ is likely a product of microorganisms present in the intestinal lumen [27]. The predicted biliary excretion (74\% of the dose) together with intestinal enterocyte efflux back to lumen (16\% of the dose) is 2 -fold higher than the reported $\mathrm{CO}_{2}$ level in vivo at the same dose.

The key parameters that influence the predicted plasma $\mathrm{AUC}_{0-24 \mathrm{~h}}$ of all quercetin metabolites in human at a dose of $0.4 \mathrm{mg} / \mathrm{kg}$ bw quercetin were assessed and are shown in Figure 3.6. The plasma $\mathrm{AUC}_{0-24 \mathrm{~h}}$ of quercetin metabolites are mainly influenced by parameters determining concentration in the liver, namely liver volume, biliary excretion rate constant and S9 protein content in liver. The blood/plasma ratio is also found to have a great influence on the prediction as it determines the concentration of metabolites in plasma. The kinetic parameters for formation of mono-conjugates especially in the liver as well as the correction factors for the $V_{\max }$ and the $K_{\mathrm{m}}$ for formation of di- and tri-conjugates, have an influence on the plasma $\mathrm{AUC}_{0-24 \mathrm{~h}}$ of quercetin metabolites. Other parameters including partition coefficients and blood flow rates to tissue do not have an effect on the predicted plasma $\mathrm{AUC}_{0-24 \mathrm{~h}}$ of quercetin metabolites.

\subsubsection{Model simulations}

Figure 3.7 shows the predicted regiospecificity of the six metabolites with the highest plasma $\mathrm{AUC}_{0-24 \mathrm{~h}}$ at a dose of $0.4 \mathrm{mg} / \mathrm{kg}$ bw quercetin (average dietary intake) and a dose of $12 \mathrm{mg} / \mathrm{kg}$ bw quercetin (average supplementary intake), including interindividual human variation and species differences. At a dose of $0.4 \mathrm{mg} / \mathrm{kg}$ bw, quercetin-3'-O-glucuronide was predicted to be the major metabolite for 19 out of 20 individual human subjects accounting for 19 to $42 \%$ of the total plasma $\mathrm{AUC}_{0-24 \mathrm{~h}}$ (Figure 3.7A). However, individual $\mathrm{H} 0270$ (male, Caucasian, 5 months) that expressed a high level of glucuronidation at the 3-OH, was predicted to have mainly quercetin-3,3'-O-diglucuronide in the plasma accounting for $43 \%$ of the total plasma $\mathrm{AUC}_{0-24 \mathrm{~h}}$. At a dose of $12 \mathrm{mg} / \mathrm{kg}$ bw quercetin, quercetin-3'-O-glucuronide becomes the major metabolite for all individuals (Figure 3.7B) due to saturation of other reactions at higher dose levels.

Comparison of the predicted levels of the major circulating metabolites of quercetin in humans to those previously predicted for male rats revealed major species differences in circulating metabolites [12]. In male rats a di- and tri-conjugate of quercetin containing a glucuronic acid, sulfate and/or methyl moiety were predicted to be the major circulating metabolites of quercetin at both average dietary and supplementary intake. The differences 


\section{Chapter 3}

between male rats and human in regiospecificity of circulating metabolites were especially a result of the catalytic efficiency and regioselectivity, which the primary metabolites are formed (Table 3.7).

Table 3.7 Kinetic parameters used in the PBK model for quercetin in human and male rat

\begin{tabular}{|c|c|c|c|c|c|c|}
\hline Parameter & \multicolumn{2}{|c|}{ Human } & \multicolumn{4}{|c|}{ Male rat ${ }^{1}$} \\
\hline Uptake rate of quercetin (h-1) [20] & \multicolumn{3}{|l|}{5.32} & \multicolumn{3}{|l|}{5.32} \\
\hline Biliary excretion (h-1) & \multicolumn{3}{|l|}{27} & \multicolumn{3}{|l|}{16} \\
\hline Urinary excretion $(\mathrm{h}-1)$ & \multicolumn{3}{|l|}{15} & \multicolumn{3}{|l|}{6.5} \\
\hline Intestinal enterocyte efflux $(\mathrm{l} / \mathrm{h})$ & \multicolumn{3}{|l|}{5} & \multicolumn{3}{|l|}{0.17} \\
\hline \multicolumn{7}{|l|}{ Kinetics for mono-conjugates } \\
\hline Small intestine & $K_{\mathrm{m}}^{2}$ & $V_{\max }^{3}$ & $\begin{array}{l}\text { catalytic } \\
\text { efficiency }^{4}\end{array}$ & $K_{\mathrm{m}}^{2}$ & $V_{\max }^{3}$ & $\begin{array}{l}\text { catalytic } \\
\text { efficiency }^{4}\end{array}$ \\
\hline quercetin-7-O-glucuronide & $0.84 \pm 0.23$ & $0.14 \pm 0.01$ & 0.11 & $0.28 \pm 0.07$ & $1.89 \pm 0.1$ & 4.62 \\
\hline quercetin-3-O-glucuronide & $0.56 \pm 0.15$ & $0.34 \pm 0.02$ & 0.42 & $0.28 \pm 0.07$ & $0.47 \pm 0.02$ & 1.15 \\
\hline quercetin-3'-O-glucuronide & $0.20 \pm 0.05$ & $0.61 \pm 0.03$ & 2.09 & $1.14 \pm 0.24$ & $0.92 \pm 0.06$ & 0.55 \\
\hline quercetin-4'-O-glucuronide & $0.46 \pm 0.12$ & $0.37 \pm 0.02$ & 0.55 & $\mathrm{nd}^{5}$ & nd & \\
\hline quercetin-7-O-sulfate & $0.41 \pm 0.14$ & $0.02 \pm 0.001$ & 0.03 & nd & nd & \\
\hline quercetin-3'-O-sulfate & $1.17 \pm 0.35$ & $0.11 \pm 0.01$ & 0.44 & $0.11 \pm 0.03$ & $0.01 \pm 0.001$ & 0.07 \\
\hline \multicolumn{7}{|l|}{ Liver } \\
\hline quercetin-7-O-glucuronide & $1.37 \pm 0.04$ & $0.66 \pm 0.04$ & 4.13 & $8.08 \pm 2.6$ & $6.11 \pm 0.83$ & 6.49 \\
\hline quercetin-3-O-glucuronide & $0.87 \pm 0.14$ & $0.39 \pm 0.02$ & 3.85 & nd & nd & \\
\hline quercetin-3'-O-glucuronide & $0.21 \pm 0.08$ & $0.40 \pm 0.02$ & 20 & $1.13 \pm 0.29$ & $1.46 \pm 0.09$ & 11 \\
\hline quercetin-4'-O-glucuronide & nd & nd & & $0.12 \pm 0.04$ & $0.66 \pm 0.02$ & 47 \\
\hline quercetin-7-O-sulfate & nd & nd & 0.25 & $0.47 \pm 0.2$ & $0.04 \pm 0.004$ & 0.73 \\
\hline quercetin-3'-O-sulfate & $0.35 \pm 0.07$ & $0.01 \pm 0.0004$ & 0.18 & $0.27 \pm 0.07$ & $0.14 \pm 0.01$ & 4.45 \\
\hline 3'-O-methylquercetin & $0.75 \pm 3.12$ & $0.03 \pm 0.002$ & 0.08 & $24 \pm 3.39$ & $0.46 \pm 0.02$ & 0.16 \\
\hline 4'-O-methylquercetin & $0.75 \pm 3.12$ & $0.02 \pm 0.001$ & 0.11 & $24 \pm 3.39$ & $0.23 \pm 0.01$ & 0.08 \\
\hline \multicolumn{7}{|l|}{ Correction factor for $K_{\mathrm{m}}$} \\
\hline sulfation of QG or QGM & \multicolumn{3}{|l|}{5} & \multicolumn{3}{|l|}{2.2} \\
\hline methylation of QG or QGS & \multicolumn{3}{|l|}{1.6} & \multicolumn{3}{|l|}{1} \\
\hline glucuronidation of QG or QGM & \multicolumn{3}{|l|}{41} & \multicolumn{3}{|l|}{13} \\
\hline methylation of QdiG & \multicolumn{3}{|l|}{2} & \multicolumn{3}{|l|}{87} \\
\hline \multicolumn{7}{|l|}{ Correction factor for $V_{\max }$} \\
\hline sulfation of QG or QGM & \multicolumn{4}{|c|}{1.7} & \multicolumn{2}{|l|}{1.5} \\
\hline methylation of QG or QGS & \multicolumn{4}{|c|}{3} & \multicolumn{2}{|l|}{1} \\
\hline glucuronidation of QG or QGM & \multicolumn{4}{|c|}{20} & \multicolumn{2}{|l|}{12} \\
\hline methylation of QdiG & \multicolumn{2}{|r|}{2.9} & & & 31 & \\
\hline $\begin{array}{l}\text { Kinetic parameters for male rat were } \\
K_{\mathrm{m}(\text { app })} \text { expressed as } \mu \mathrm{M} \text {. } \\
V_{\max (\text { app })} \text { expressed as } \mathrm{nmol} / \mathrm{min} / \mathrm{mg} \\
\text { catalytic efficiency }\left(\mathrm{Scaled} V_{\mathrm{max}} / K_{\mathrm{m}}\right. \\
\text { app } /(1000 \mathrm{nmol} / \mu \mathrm{mol}) *(60 \mathrm{~min} / \mathrm{h}) *(\mathrm{n} \\
\mathrm{nd}=\text { not detect able. }\end{array}$ & $\begin{array}{l}\text { btained from } \\
\text { protein. } \\
\text { )) expressed a } \\
\text { S9/g tissue): }\end{array}$ & $\begin{array}{l}\text { onpawa et al. } \\
\text { /h/g tissue; sc } \\
.4 \text { and } 143 \text { m }\end{array}$ & $V_{\max }(\mu \mathrm{mol}$ & h/g tissue) c & $\begin{array}{l}\text { lated from } V_{\mathrm{m}} \\
\text { n liver. }\end{array}$ & \\
\hline
\end{tabular}




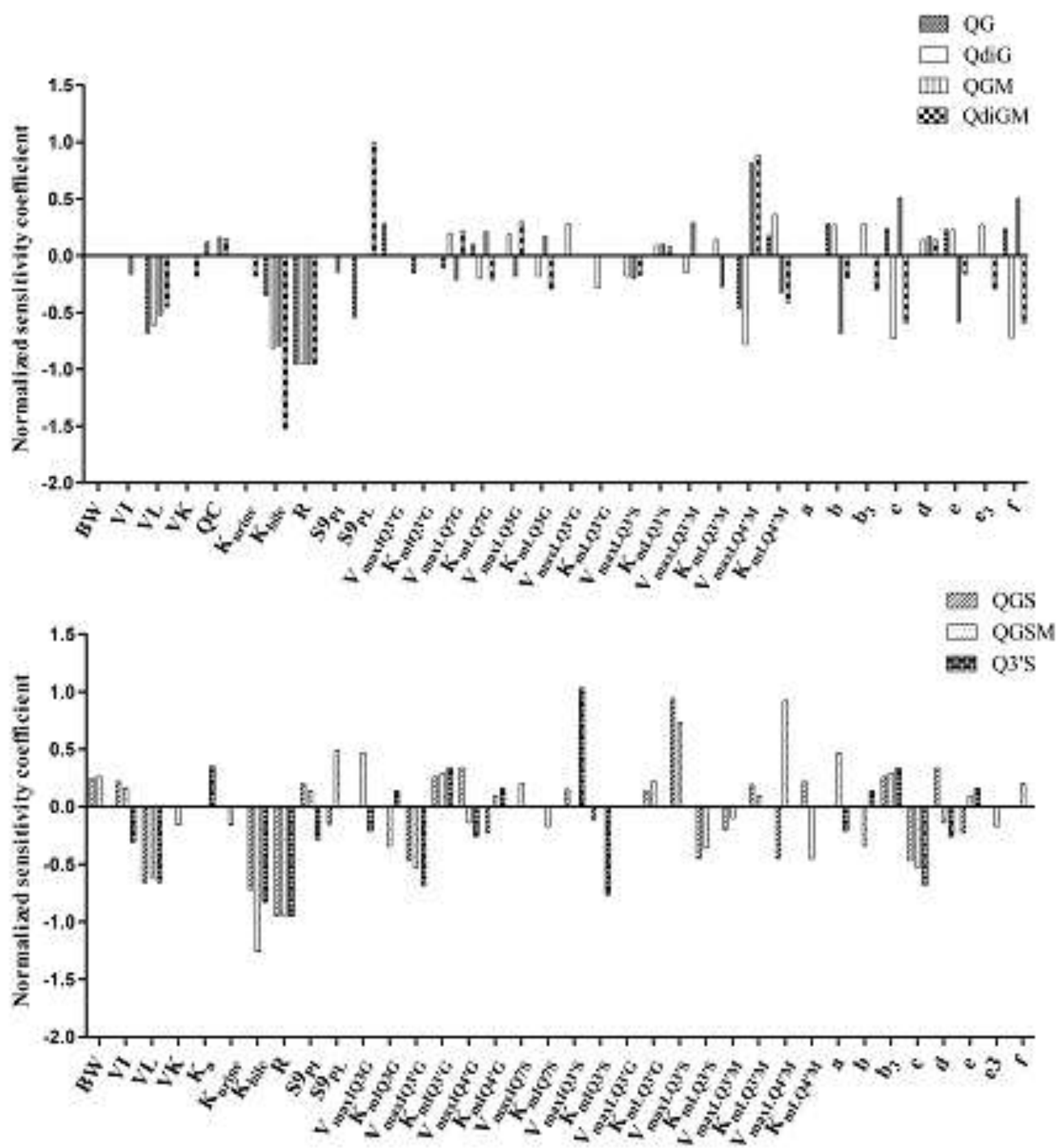

Figure 3.6 Sensitivity analysis of the predicted plasma AUC0-24h of quercetin monoglucuronides $(\mathrm{QG})$, quercetin diglucuronides (QdiG), quercetin glucuronide methyl conjugates (QGM), quercetin glucuronide sulfate conjugates (QGS), quercetin diglucuronide methyl conjugates (QdiGM), quercetin glucuronide sulfate methyl conjugates (QGSM), and quercetin-3'-O-sulfate (Q3'S) in human exposed to $0.4 \mathrm{mg} / \mathrm{kg}$ bw quercetin. The parameters are stand for: $\mathrm{BW}=$ body weight, $\mathrm{VTi}=$ tissue volume (Ti $=\mathrm{I}$ (small intestine), $\mathrm{L}$ (liver), $\mathrm{K}$ (kidney)), $\mathrm{QC}=$ cardiac output, $\mathrm{K}_{\mathrm{a}}=$ uptake rate of quercetin, $\mathrm{K}_{\text {urine }}=$ urinary excretion rate constant, $\mathrm{K}_{\text {bile }}=$ biliary excretion rate constant, $\mathrm{R}=$ blood/plasma ratio, $\mathrm{S} 9 \mathrm{PI}=$ small intestinal S9 protein content, S9PL = liver S9 protein content, $V_{\max }$ and $K_{\mathrm{m}}=$ the maximum rate of formation and the Michaelis-Menten constant for formation of metabolite ( $\mathrm{G}$ (glucuronide), $\mathrm{S}$ (sulfate), M (methylate), and correction factors for the reduction of $V_{\max }$ for sulfate-conjugates of QG or QGM (a), methylate-conjugates of QG or QGS (b), methylate-conjugated of QdiG $\left(b_{3}\right)$, glucuronide-conjugates of QG or QGM (c) and for the induction of $K_{\mathrm{m}}$ for sulfate-conjugates of QG or QGM (d), methylate-conjugates of QG or QGS (e), methylate-conjugated of QdiG $\left(\mathrm{e}_{3}\right)$, glucuronideconjugates of QG or QGM (f). 
Chapter 3
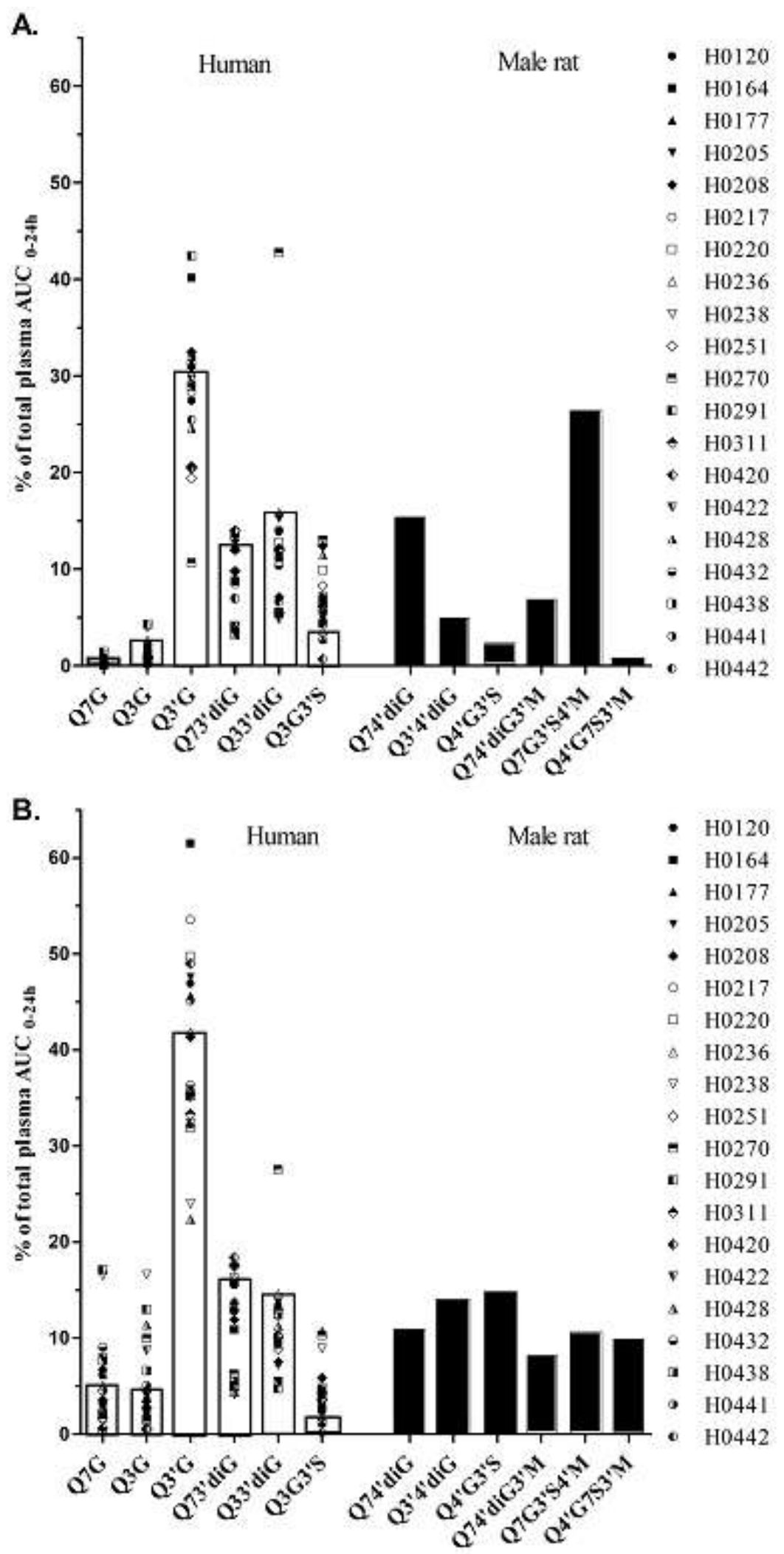

Figure 3.7 Prediction on regiospecificity of the major circulating metabolites of quercetin in (individual) human subjects as compared with male rat [12] at a dose of 0.4 (A) and $12 \mathrm{mg} / \mathrm{kg}$ bw quercetin $(\mathrm{B})$; quercetin $(\mathrm{Q})$, glucuronide $(\mathrm{G})$, sulfate $(\mathrm{S})$ and methyl conjugate $(\mathrm{M})$. Data were expressed as \% of total plasma $\mathrm{AUC}_{0-24 \mathrm{~h}}$. The bar and dots present the prediction obtained from the average and individual models. 


\subsection{Discussion}

Flavonoid aglycones have been intensively used in vitro to elucidate its mode of actions underlying the health beneficial effects of flavonoids. However, in vivo effects of flavonoids are ultimately dependent on the bioavailability of flavonoid aglycones and its metabolites. In the present study, (individual) human PBK models for quercetin were defined by integrating kinetic data obtained from in vitro incubations with (individual) human tissue fractions, providing information on the nature and time-dependent formation of quercetin and its metabolites in humans and interindividual human variation herein. The outcomes obtained allowed to tentatively predict the regiospecificity of the major circulating metabolites in human, which were compared to those previously predicted with a PBK model for quercetin in male rat to elucidate species differences [12]. The focus was on metabolites that formed by intestinal and liver tissue. Potential metabolic conversion by gut microbiota, was indirectly accounted for by simulating biliary excretion and intestinal enterocyte efflux back to lumen.

The bioavailability of quercetin aglycone in (individual) human subjects was predicted to be limited ranging from 0.001 to $0.04 \%$ of the dose at an average dietary intake of 0.4 $\mathrm{mg} / \mathrm{kg}$ bw quercetin. This result is in agreement with results from previous studies of Gugler et al. [28] and Graefe et al. reporting that no quercetin aglycone can be detected in human plasma after consumption of 50 to $60 \mathrm{mg} / \mathrm{kg}$ bw quercetin or $2.2 \mathrm{mg} / \mathrm{kg}$ bw Q4'Gly [29]. The low bioavailability of quercetin aglycone is due to extensive first pass metabolism of quercetin in the body, especially via glucuronidation as $\sim 96 \%$ of the dose was predicted in the present study to be converted to monoglucuronides within $1.5 \mathrm{~h}$.

Quercetin-3'-O-glucuronide was predicted to be the major circulating metabolite of quercetin in most individual humans both at a dose relevant to average dietary and supplementary intake of 0.4 and $12 \mathrm{mg} / \mathrm{kg}$ bw, respectively. In one individual, however, a glucuronide at the 3-position of quercetin-3'-O-glucuronide was the major circulating metabolite, indicating a swift further conversion of quercetin-3'-O-glucuronide in this individual. The importance of quercetin-3'-O-glucuronide and its further conjugates as major metabolites of quercetin is supported by a study of Mullen et al. in which quercetin-3'-Oglucuronide and di- and tri-conjugates containing a glucuronide moiety were the major metabolites in the urine after consumption of onions [10]. However, these metabolites were not observed to be the major metabolites in the plasma, which was observed to mainly contain quercetin-3'-O-sulfate [10]. A more recent study by Lee et al., revealed that quercetin conjugates containing glucuronide moiety are the most important metabolites present in 


\section{Chapter 3}

human plasma, when quantified with LC-MS in positive ion mode [9]. In the study of Lee et al. the regiospecificity of these metabolites was not determined, and the importance of especially quercetin-3'-O-glucuronide as a major circulating can therefore not be confirmed based on this study.

Interindividual human variation was observed in the kinetic constants for glucuronidation, sulfation and methylation as determined with the incubations performed with the 20 individual human liver fractions of the present study (Table 3.4-3.6). These differences lead to a variation in the plasma concentrations of quercetin metabolites including a 4-fold variation in the predicted level of the major circulating metabolite, quercetin-3'-Oglucuronide. This variation was mainly a result of differences in glucuronidation efficiency at positions besides the 3 '-position, rather than interindividual human variation for further conjugation of this metabolite to sulfate or methyl conjugates or formation of $3^{\prime}-\mathrm{O}$ glucuronide itself. For example, the lowest plasma concentration of quercetin-3'-Oglucuronide was observed for individual H0270, who displayed the highest catalytic efficiency for further conjugation of quercetin-3'-O-glucuronide at the 3-position, resulting in quercetin-3,3'-O-diglucuronide as the major circulating metabolite for this individual. These results may suggest that especially the genetic- and lifestyle factors influencing the expression and/or activity of UGT enzymes involved in quercetin glucuronidation, including UGT1A1, UGT1A3, UGT1A8, UGT1A9, and UGT2B7 may influence the regiospecificity of circulating metabolites [16].

With the developed PBK models for quercetin in (individual) human subjects and the previously defined model for rats, also species differences in metabolism of quercetin could be evaluated. Whereas the major predicted circulating metabolites of quercetin in humans are monoglucuronides and predominantly quercetin-3'-O-glucuronide, the major circulating metabolites in rats were di- and tri-conjugates containing glucuronic acid, sulfate and/or methyl moiety (Figure 3.7). These differences are partly due to a 2.3-fold higher metabolic conversion in rats as compared with humans, resulting in a faster further conversion of the monoglucuronides. A higher rate of glucuronidation in rats compared with human is more commonly observed. For example, Furukawa et al., reported that glucuronidation of quercetin in rat is 2.2-fold more efficient than in human based on the catalytic efficiencies obtained from in vitro incubations with rat and human intestinal microsomes [30]. Glucuronidation of related flavonoids, including hesperetin, kaempferol, genistein, daidzein, and glycitein has been reported to be more efficient in rat than in human as observed in in vitro incubations 
Inter- and intraspecies differences in metabolic plasma profiles of quercetin

with tissue fractions [31-34]. Studies on the glucuronidation of other compounds including buprenorphine, entacapone, ezetimibe, mycophenolic acid, and tolcapone further support that rat has higher glucuronidating activities than human based on the catalytic efficiencies obtained from in vitro incubations with rat and human intestinal microsomes [30]. Also species differences in the tentatively identified regiospecificity of circulating metabolites were observed, as rats mainly show primary glucuronidation at the $4^{\prime}-\mathrm{OH}$ and humans at the $3^{\prime}-\mathrm{OH}$ and di- and tri-conjugates building further on these different mono-conjugates in both species. This differences in conversion of flavonoids may be a result of differences in the size and shape of the binding pocket of different enzymes involved in the reactions [35]. Glucuronidation of quercetin in humans has been identified to be catalyzed by UGT1A1, UGT1A8 and UGT1A9 based on incubations with recombinant enzymes [16], whereas glucuronidation in rats has been linked to UGT1A1 and UGT1A7 activity in an in vitro study with rat tissue fractions [36]. Especially UGT1A1 has been reported to preferentially convert flavones at the $3^{\prime}-\mathrm{OH}$ (the primary glucuronide formed in humans in the present study) [35]. A difference in contribution of this enzyme to the total glucuronidation activity between the two species might therefore explain the observed differences in primary metabolites that are formed and consequently the secondary and tertiary metabolites that build upon these primary glucuronides.

Considering that the nature and the position of flavonoid conjugation could be a key factor determining biological activity of flavonoids in vivo [37], the observed differences between individual human subjects in circulating metabolites could indicate critical differences in sensitivity to health beneficial effects. In addition, the differences between rats and humans may suggest that the biological effects of flavonoids observed in rats may not necessary be relevant for the human situation. At present little is known about the biological activity of flavonoid metabolites. Metabolism does not always result in a reduced biological activity of flavonoids [37]. In addition, biological activity might be a result of the extent by which the flavonoids are deconjugated to the active aglycone in a tissue [38, 39]. The results obtained with the PBK model allow to identify which metabolites will be relevant to study for their biological activity or to use in incubations to determine deconjugation, which are for humans predominantly quercetin-3'-O-glucuronide and conjugates of this metabolite.

Overall, the newly developed PBK model adequately predicted overall plasma concentrations of quercetin and its metabolites including mono-, di-, and tri-conjugates and provides an indication of the nature of the circulating metabolites of quercetin in plasma of 


\section{Chapter 3}

humans and interindivdiual human variation herein. Significant species differences occur in the major circulating metabolites of quercetin in the systemic circulation indicating that rat is not an adequate model to study effects of quercetin in man. The developed PBK models can also be used to guide experimental design of in vitro experiments with flavonoids, especially to take into account the relevance of metabolism and the contribution of metabolites to the biological activity of flavonoids in humans.

\section{Conflict of interest}

The authors state no conflict of interest.

\section{Acknowledgements}

This research was financially supported by the Ministry of Science and Technology of Thailand through a Royal Thai Government Scholarship awarded to Rungnapa Boonpawa for conducting her $\mathrm{PhD}$ thesis in The Netherlands. 
Inter- and intraspecies differences in metabolic plasma profiles of quercetin

\section{References}

1. Neuhouser, M. L., Review: dietary flavonoids and cancer risk: evidence from human population studies. Nutrition and Cancer, 2004. 50(1): p. 1-7.

2. Perez-Vizcaino, F. and J. Duarte, Flavonols and cardiovascular disease. Molecular Aspects of Medicine, 2010. 31(6): p. 478-494.

3. Larson, A., J. D. Symons, and T. Jalili, Quercetin: A treatment for hypertension? - A review of efficacy and mechanisms. Pharmaceuticals, 2010. 3(1): p. 237-250.

4. Aguirre, L., et al., Beneficial effects of quercetin on obesity and diabetes. The Open Nutraceuticals, 2011. 4: p. 189-198.

5. de Boer, V. C. J., et al., Tissue distribution of quercetin in rats and pigs. The Journal of Nutrition, 2005. 135(7): p. 1718-1725.

6. Németh, K., et al., Deglycosylation by small intestinal epithelial cell $\beta$-glucosidases is a critical step in the absorption and metabolism of dietary flavonoid glycosides in humans. European Journal of Nutrition, 2003. 42(1): p. 29-42.

7. Day, A. J., et al., Absorption of quercetin-3-glucoside and quercetin-4'-glucoside in the rat small intestine: the role of lactase phlorizin hydrolase and the sodium-dependent glucose transporter. Biochemical Pharmacology, 2003. 65(7): p. 1199-1206.

8. Graf, B. A., et al., Disposition and metabolism of $\left[2-{ }^{14} \mathrm{C}\right]$ quercetin-4'-glucoside in rats. Drug Metabolism and Disposition, 2005. 33(7): p. 1036-1043.

9. Lee, J., et al., UHPLC-(ESI)QTOF MS/MS profiling of quercetin metabolites in human plasma postconsumption of applesauce enriched with apple peel and onion. Journal of Agricultural and Food Chemistry, 2012. 60(34): p. 8510-8520.

10. Mullen, W., C. A. Edwards, and A. Crozier, Absorption, excretion and metabolite profiling of methyl-, glucuronyl-, glucosyl- and sulpho-conjugates of quercetin in human plasma and urine after ingestion of onions. British Journal of Nutrition, 2006. 96: p. 107-116.

11. Day, A. J., et al., Human metabolism of dietary flavonoids: Identification of plasma metabolites of quercetin. Free Radical Research, 2001. 35(6): p. 941-952.

12. Boonpawa, R., et al., A physiologically based kinetic (PBK) model describing plasma concentrations of quercetin and its metabolites in rats. Biochemical Pharmacology, 2014. 89(2): p. 287-299.

13. Fisher, M. B., et al., In vitro glucuronidation using human liver microsomes and the poreforming peptide alamethicin. Drug Metabolism and Disposition, 2000. 28(5): p. 560-566.

14. Diez-Roux, G. and A. Ballabio, Sulfatases and human disease. Annual Review of Genomics and Human Genetics, 2005. 6(1): p. 355-379.

15. Parenti, G., G. Meroni, and A. Ballabio, The sulfatase gene family. Current Opinion in Genetics \& Development, 1997. 7(3): p. 386-391.

16. Boersma, M. G., et al., Regioselectivity of phase II metabolism of luteolin and quercetin by UDP-glucuronosyl transferases. Chemical Research in Toxicology, 2002. 15(5): p. 662-670.

17. van der Woude, H., et al., Identification of 14 quercetin phase II mono- and mixed conjugates and their formation by rat and human phase II in vitro model systems. Chemical Research in Toxicology, 2004. 17(11): p. 1520-1530.

18. Walle, T., et al., Quercetin glucosides are completely hydrolyzed in ileostomy patients before absorption. The Journal of Nutrition, 2000. 130(11): p. 2658-2661.

19. Petri, N., et al., Absorption/metabolism of sulforaphane and quercetin, and regulation of phase II enzymes, in human jejunum in vivo. Drug Metabolism and Disposition, 2003. 31(6): p. 805-813. 
20. Chen, X., et al., Pharmacokinetics and modeling of quercetin and metabolites. Pharmaceutical Research, 2005. 22(6): p. 892-901.

21. Brown, R. P., et al., Physiological parameter values for physiologically based pharmacokinetic models. Toxicology and Industrial Health, 1997. 13(4): p. 407-484.

22. DeJongh, J., H. J. M. Verhaar, and J. L. M. Hermens, A quantitative property-property relationship (QPPR) approach to estimate in vitro tissue-blood partition coefficients of organic chemicals in rats and humans. Archives of Toxicology, 1997. 72(1): p. 17-25.

23. Hong, Y.-J. and A. E. Mitchell, Metabolic profiling of flavonol metabolites in human urine by liquid chromatography and tandem mass spectrometry. Journal of Agricultural and Food Chemistry, 2004. 52(22): p. 6794-6801.

24. Walle, T., U. K. Walle, and P. V. Halushka, Carbon dioxide is the major metabolite of quercetin in humans. The Journal of Nutrition, 2001. 131(10): p. 2648-2652.

25. Simcyp. Simcyp prediction tools-fu. [Website]. Available from: https://members.simcyp.com/account/tools/fu/ [Accessed on: 07-07-2014]. 2014.

26. Simcyp. Simcyp prediction tools - blood to plasma partition ratio (B/P).[Website]. Available from: https://members.simcyp.com/account/tools/BP/ [Accessed on: 7-7-2014]. 2014 [cited 2014.

27. Ueno, I., N. Nakano, and I. Hirono, Metabolic fate of [14C] quercetin in the ACl rat. Japanese Journal of Experimental Medicine, 1983. 53(1): p. 41-50.

28. Gugler, R., M. Leschik, and H. J. Dengler, Disposition of quercetin in man after single oral and intravenous doses. 1975. 9(2-3): p. 229-234.

29. Graefe, E. U., et al., Pharmacokinetics and bioavailability of quercetin glycosides in humans. The Journal of Clinical Pharmacology, 2001. 41(5): p. 492-499.

30. Furukawa, T., et al., Species differences in intestinal glucuronidation activities between humans, rats, dogs and monkeys. Xenobiotica, 2014. 44(3): p. 205-216.

31. Brand, W., et al., Phase II Metabolism of hesperetin by individual UDPglucuronosyltransferases and sulfotransferases and rat and human tissue samples. Drug Metabolism and Disposition, 2010. 38(4): p. 617-625.

32. Otake, Y., F. Hsieh, and T. Walle, Glucuronidation versus oxidation of the flavonoid galangin by human liver microsomes and hepatocytes. Drug Metabolism and Disposition, 2002. 30(5): p. 576-581.

33. Barve, A., et al., Metabolism, oral bioavailability and pharmacokinetics of chemopreventive kaempferol in rats. Biopharmaceutics \& drug disposition, 2009. 30(7): p. 356-365.

34. Islam, M. A., et al., Conversion of major soy isoflavone glucosides and aglycones in in vitro intestinal models. Molecular Nutrition \& Food Research, 2014. 58(3): p. 503-515.

35. Wu, B., et al., Regioselective sulfation and glucuronidation of phenolics: insights into the structural basis of conjugation. Current drug metabolism, 2011. 12(9): p. 900-916.

36. Bolling, B. W., et al., Microsomal quercetin glucuronidation in rat small intestine depends on age and segment. Drug Metabolism and Disposition, 2011.

37. Beekmann, K., et al., A state-of-the-art overview of the effect of metabolic conjugation on the biological activity of flavonoids. Food \& Function, 2012. 3(10): p. 1008-1018.

38. Galindo, P., et al., Glucuronidated quercetin lowers blood pressure in spontaneously hypertensive rats via deconjugation. PLoS ONE, 2012. 7(3): p. e32673.

39. Bartholomé, R., et al., Deconjugation kinetics of glucuronidated phase II flavonoid metabolites by $\beta$-glucuronidase from neutrophils. Drug Metabolism and Pharmacokinetics, 2010. 25(4): p. 379-387. 
Inter- and intraspecies differences in metabolic plasma profiles of quercetin 
Chapter 3 


\section{CHAPTER 4}

In vitro-in silico based analysis of the dose-dependent in vivo estrogenicity of the soy phytoestrogen genistein in humans

Rungnapa Boonpawa,

Albertus Spenkelink,

Ans Punt,

Ivonne M. C. M. Rietjens

Submission 


\begin{abstract}
In vivo estrogenicity of genistein and its glycoside genistin is still under debate. The present study aimed to develop a physiologically based kinetic (PBK) model that provides insight in dose-dependent plasma concentrations of genistein aglycone and its metabolites and enables prediction of in vivo estrogenic effective dose levels of genistein and genistin in humans. A PBK model for genistein and genistin in humans was developed based on in vitro metabolic parameters. The model obtained was used to translate in vitro estrogenic concentration-response curves of genistein to in vivo estrogenic dose-response curves for intake of genistein and genistin. The model predicted that genistein-7-O-glucuronide was the major circulating metabolite and that levels of the free aglycone were generally low $(0.5-17 \%$ of total plasma genistein at oral doses from $0.01-50 \mathrm{mg} / \mathrm{kg}$ bw. The predicted in vivo $\mathrm{BMD}_{05}$ (benchmark dose for 5\% response) values for estrogenicity varied between 0.03 and 4.56 $\mathrm{mg} / \mathrm{kg}$ bw genistein. For genistin, these values were 1.3-fold higher. These values are in line with reported human data and show that estrogenic responses can be expected at an Asian dietary and a supplementary intake, while intake resulting from a Western diet may not be effective. The present study shows how plasma concentrations of genistein and its metabolites and estrogenic dose levels of genistein in humans can be predicted by combining in vitro estrogenicity with PBK model based reverse dosimetry, eliminating the need for human intervention studies.
\end{abstract}




\subsection{Introduction}

Isoflavones are a common group of polyphenols and are generally known as phytoestrogens as they can exert weak estrogenic potencies due to their basic structural similarity to endogenous estrogen [1]. Isoflavones have been reported to reduce the incidence of mammary tumors in experimental animal models [2] and in observational epidemiologic studies [3]. However, there is also evidence suggesting that isoflavones can stimulate cell proliferation of breast cancer cells in vitro [4-8] and in ovariectomized animal models of breast cancer [9] in a dose-dependent manner. These findings raised a concern about the safety of these isoflavones, especially in patients with existing estrogen-sensitive tumors and woman at risk of developing breast cancer. A possible mode of action underlying these positive and negative effects of isoflavones might be the modulation of estrogen receptor (ER) signaling as agonist or antagonist depending on the endogenous concentration of ERs [1]. ERs consist of two major isoforms; ER $\alpha$ that drives proliferation and ER $\beta$ that inhibits proliferation and stimulates apoptosis [1]. These two receptors moderate each other's effects and therefore their absolute concentration and ratio within a tissue may influence the cellular response toward different xenoestrogens [10]. A recent scientific opinion of the European Food Safety Authority (EFSA) concluded that "there is no indication for adverse effects on the mammary gland, uterus, and thyroid function and no genotoxic concern in postmenopausal women taking 30-900 mg/day isoflavones supplements for 3-36 months, but that there are insufficient data to assess the risk of isoflavone supplements in post-menopausal women with a current diagnosis or history of estrogen-dependent cancer" [11].

Genistein is the major isoflavone in soy occurring in plants mainly in its glycosylated form, genistin (genistein-7-O-glucoside) [12], while fermentation increases the aglycone content in soy-based products $[12,13]$. The metabolic pathways of genistin and genistein are shown in Figure 4.1. The glycosylated genistin is hydrolyzed to genistein aglycone prior to absorption in the intestine by brush-border lactase phlorizin hydrolase [14] or by gut microbiota [15]. Upon absorption, genistein is extensively metabolized via glucuronidation and sulfation in both intestinal cells and liver cells. Most of the absorbed genistein is present in the systemic circulation in the form of glucuronides, sulfates, and mixed conjugates at the 4'- and/or 7-position [16-18].

The genistein conjugates tested so far, including genistein-7-O-glucuronide (G-7G), genistein-7-O-sulfate (G-7S), and genistein-4'-O-sulfate (G-4'S), appeared inactive in assays 
for estrogenicity leading to the conclusion that the action of the soy phytoestrogen genistein will be mainly related to the plasma and tissue concentration of the free aglycone $[13,19]$.

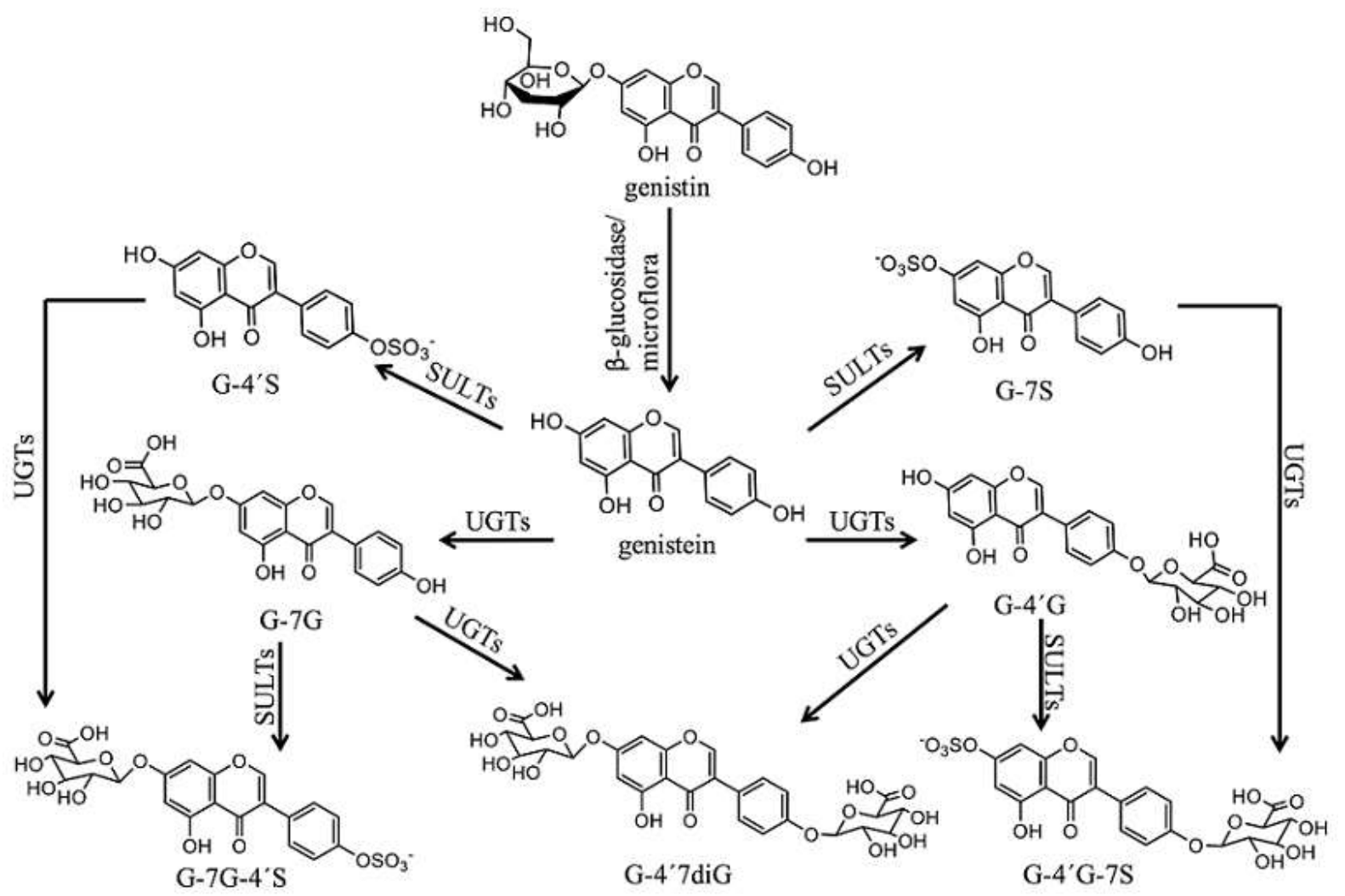

Figure 4.1 Proposed metabolic pathways of genistin and genistein in humans [16-18].

Available data on the estrogenic activity of genistein in humans under realistic exposure scenario's are contradicting and inconclusive. In some human studies, genistein and other isoflavones have been reported to exert an estrogenic response in especially healthy premenopausal women [20-23]. Contradictory, some human studies report no estrogenic response of genistein/isoflavones in healthy as well as breast cancer women even at supplementary doses [2, 24-26]. Given that the estrogenicity of genistein might strongly depend on the plasma levels of its aglycone, it is of importance to better understand the absorption, distribution, metabolism, and excretion (ADME) of genistein in humans and the actual plasma levels of genistein and its various metabolites in human subjects. The aim of the present study was to develop a physiologically based kinetic (PBK) model that provides insight in the dose-dependent plasma concentration of genistein aglycone and its metabolites and enables prediction of the in vivo dose-response curves for estrogenicity of genistein and genistin in humans based on in vitro concentration-response curves. The outcomes obtained can be used to determine whether and if so at what oral dose levels genistein or genistin may elicit estrogenic effects in vivo. 


\subsection{Materials and Methods}

\subsubsection{Materials}

Pooled mixed-gender human small intestine and liver S9 fractions were purchased from Xenotech (Lenexa, USA). Uridine 5'-diphosphoglucuronide acid (UDPGA, purity 98\%), 3'-phosphoadenosine 5'-phosphosulfate (PAPS, purity 65\%), Tris, alamethicin (from

Trichoderma viride), D-saccharic acid 1,4-lactone monohydrate, and $\beta$ glucuronidase/sulfatase (from Helix pomatia) were purchased from Sigma-Aldrich (Steinheim, Germany). Hydrochloric acid (fuming 37\%), magnesium chloride, trifluoroacetic acid (for spectroscopy), and DMSO (99.9\%) were purchased from VWR International (Darmstadt, Germany). Acetonitrile (chromatography grade) was purchased from Biosolve (Valkenswaard, The Netherlands). Genistein (>99\%) was purchased from LC Laboratories (Woburn, MA, USA). G-7G was purchased from Extrasynthese (Geny, France). G-7S, G-4'G, genistein-4' ,7-O-diglucuronide (G-4’7diG), and genistein-7-O-glucuronide-4' -O-sulfate (G7G-4’S) were purchased from Toronto Research Chemicals (Toronto, Canada).

4.2.2 Enzymatic conjugation of genistein and genistein mono-conjugates with pooled human tissue fractions

Kinetic constants for sulfation of genistein and conjugation of the mono-conjugates G-7G, G-4' G, and G-7S to di-conjugates, needed as parameters in the PBK models, were obtained from in vitro incubations with pooled mixed-gender human small intestine and liver S9 fractions based on methods described by Islam et al. [12] with some modifications. Use of mixed-gender human tissue fractions was considered adequate to represent both male and female individuals, because no significant gender differences on maximum concentration $\left(\mathrm{C}_{\max }\right)$, area under the -concentration-time curve (AUC), and time to read $\mathrm{C}_{\max }\left(\mathrm{T}_{\max }\right)$ in plasma of total genistein (genistein aglycone and its metabolites) between male and female human volunteers following consumption of soy foods were observed [27].

For glucuronidation, $100 \mu \mathrm{l}$ incubation mixtures (final volume) were prepared containing $10 \mathrm{mM} \mathrm{MgCl} 2,20 \mathrm{mM}$ D-saccharic acid 1,4-lactone (pH 7.4), 10 mM UDPGA, and human tissue fractions in $50 \mathrm{mM}$ Tris- $\mathrm{HCl}(\mathrm{pH}$ 7.4). D-saccharic acid 1,4-lactone was added to inhibit $\beta$-glucuronidase [28]. A protein content of $0.8,0.8$, and $0.2 \mathrm{mg} / \mathrm{ml}$ human small intestine S9 and of $1.2,0.8$, and $0.8 \mathrm{mg} / \mathrm{ml}$ human liver $\mathrm{S} 9$ were applied for the incubations of G-7G, G-4' G, and G-7S, respectively. The incubation mixtures were pretreated on ice with $0.025 \mathrm{mg} / \mathrm{ml}$ alamethicin added from a 200 times concentrated stock 


\section{Chapter 4}

solution in methanol for $15 \mathrm{~min}$. After $1 \mathrm{~min}$ pre-incubation at $37^{\circ} \mathrm{C}$, the reactions were initiated by adding substrate (final concentration ranging from 0.5 to $400 \mu \mathrm{M}$ ) added from a 200 times concentrated stock solution in DMSO. The incubations of G-7G, G-4' G, and G-7S were terminated after 60, 180, and 40 min for incubations with human small intestine S9 and after 240, 240, and 240 min for incubations with human liver S9, respectively, by adding 25 $\mu 1$ ice-cold acetonitrile. Blank incubations were carried out without UDPGA. Under the specified conditions, glucuronidation was linear in time and with protein content (data not shown).

For sulfation reactions, $100 \mu \mathrm{l}$ incubation mixtures (final volume) were prepared containing $10 \mathrm{mM} \mathrm{MgCl} 2,20 \mathrm{mM}$ D-saccharic acid 1,4-lactone (pH 7.4), $0.1 \mathrm{mM}$ PAPS, and human tissue fractions in $50 \mathrm{mM}$ Tris- $\mathrm{HCl}(\mathrm{pH} 7.4)$. A protein content of $0.4,0.8$, and 0.8 $\mathrm{mg} / \mathrm{ml}$ human small intestine $\mathrm{S} 9$ or human liver $\mathrm{S} 9$ were applied for the incubations of genistein, G-7G, and G-4' G, respectively. The incubation mixtures were pre-incubated for 1 min at $37^{\circ} \mathrm{C}$ prior to an addition of substrate (final concentration ranging from 0.5 to $400 \mu \mathrm{M}$ ) added from a 200 times concentrated stock solution in DMSO to initiate the reactions. The incubations of genistein, G-7G, and G-4' G were terminated after 20, 240, and 180 min for incubations with human small intestine S9 and after 20, 240, and $240 \mathrm{~min}$ for incubations with human liver S9, respectively, by adding $25 \mu 1$ ice-cold acetonitrile. Blank incubations were carried out without PAPS. Under the specified conditions, sulfation was linear in time and with protein content (data not shown).

\subsubsection{Identification and quantification of genistein metabolites}

All samples were analyzed on a UPLC-DAD system consisting of a Waters (Milford, MA) Acquity binary solvent manager, sample manager, and photodiode array detector, equipped with a Waters Acquity UPLC BEH RP 18 column $(1.7 \mu \mathrm{m}, 2.1$ x $50 \mathrm{~mm})$. Before analysis, the samples were centrifuged at $15000 \mathrm{rpm}$ for $5 \mathrm{~min}$ at $5^{\circ} \mathrm{C}$ to precipitate proteins and $3.5 \mu \mathrm{l}$ of the supernatant was subsequently analyzed. A gradient was applied consisting of nanopure water containing $0.1 \%$ trifluoroacetic acid (eluent A) and acetonitrile (eluent B) with a flow rate of $0.6 \mathrm{ml} / \mathrm{min}$ with the following profile: $0-10 \% \mathrm{~B}(0-0.58 \mathrm{~min}), 10-15 \% \mathrm{~B}$ (0.58-2.85 min), 15-50\% B (2.85-4.28 $\mathrm{min}), 50-80 \%$ B (4.28-4.40 min), 80\% B (4.40-4.52 $\min ), 80-0 \% \mathrm{~B}(4.52-4.63 \mathrm{~min})$ and $0 \% \mathrm{~B}(4.63-5.80 \mathrm{~min})$.

Identification of the formed metabolites obtained from glucuronidation and sulfation reactions was carried out by comparing their UV-spectra and retention times with those of 
commercially available reference compounds including G-7S, G-7G-4'S, and G-4',7diG. The nature of the sulfate metabolite of G-4' $\mathrm{G}$ and the glucuronide metabolite of G-7S was confirmed by treating incubation samples with $\beta$-glucuronidase/sulfatase as there was no commercially reference compound available. To this end, $50 \mu \mathrm{l}$ of non-terminated mixtures (incubation samples) were added to $50 \mu \mathrm{l}$ of $0.1 \mathrm{M}$ sodium acetate $(\mathrm{pH} 5.0)$ containing 189 units $/ \mathrm{ml} \beta$-glucuronidase and 1 units $/ \mathrm{ml}$ sulfatase. The reactions were carried out for $2 \mathrm{~h}$ at $37^{\circ} \mathrm{C}$ and were terminated by adding $25 \mu \mathrm{l}$ of ice-cold acetonitrile.

Metabolites were quantified by integrating the peak areas at $260 \mathrm{~nm}$ and by using the calibration curves of their commercially available reference compounds. G-4' G-7S was quantified by using the calibration curve of G-7G-4'S, which was considered adequate because G-4' G-7S has the same UV-spectrum as G-7G-4'S (data not shown) and was assumed to display a similar molar extinction coefficient.

\subsubsection{Kinetic analysis}

Kinetic constants namely the apparent maximum velocity $\left(V_{\max (\mathrm{app})}\right)$ and apparent Michaelis-Menten constant $\left(K_{\mathrm{m}(\mathrm{app})}\right)$ for formation of all metabolites were determined by fitting the data to the standard Michaelis-Menten equation. For reactions demonstrating substrate inhibition, the $V_{\max }, K_{\mathrm{m}}$, and inhibition constant $\left(K_{\mathrm{i}}\right)$ were determined by fitting the data to the substrate inhibition equation. The kinetic constants were determined using GraphPad Prism version 5.04 (GraphPad Software, San Diego, California, USA).

Michaelis-Menten equation: $v=V_{\max } *[\mathrm{~S}] /\left(K_{\mathrm{m}}+[\mathrm{S}]\right)$

Substrate inhibition equation: $v=V_{\max } *[\mathrm{~S}] /\left(\left(K_{\mathrm{m}}+[\mathrm{S}]\right) *\left(1+[\mathrm{S}] / K_{\mathrm{i}}\right)\right)$

where [S] represents the substrate concentration. The values of $V_{\max }, K_{\mathrm{m}}$, and $K_{\mathrm{i}}$ were expressed in $\mathrm{nmol} / \mathrm{min} / \mathrm{mg}$ protein, $\mu \mathrm{M}$, and $\mu \mathrm{M}$, respectively.

\subsubsection{PBK model}

A PBK model for genistein and its glycoside, genistin, in humans was developed in a similar manner as previously defined for quercetin [29, 30]. The model consists of separate compartments for the intestinal lumen, small intestine, liver, kidney, rapidly perfused tissues (e.g. heart, lung, brain), and slowly perfused tissues (e.g. skin, muscle, bone) as shown in Figure 4.2. Genistin was modeled to be hydrolyzed to its aglycone, genistein, in the intestinal lumen by brush-border lactase phlorizin hydrolase with a hydrolysis clearance of $9.6 \mathrm{l} / \mathrm{h}$ as reported by Islam et al. [12]. The uptake of genistein to the small intestine compartment was 


\section{Chapter 4}

described by first-order kinetics with an absorption rate constant of $5.02 \mathrm{~h}^{-1}$ as reported by Steensma et al. [31]. The genistein aglycone was not modeled to be excreted since only a trace amount of unconjugated genistein is detected in human urine following oral uptake of genistein $[17,18]$. The kinetic parameters for excretion of genistein conjugates via urine, bile, and intestinal efflux back to intestinal lumen were obtained by fitting the predicted dosedependent plasma concentration of total genistein (genistein aglycone plus metabolites) with available experimental plasma human data [16-18, 32,33] resulting in an average value of 4 $\mathrm{h}^{-1}, 2 \mathrm{~h}^{-1}$, and $0.1 \mathrm{l} / \mathrm{h}$, respectively. Genistein was modeled to undergo metabolic conversion via glucuronidation and sulfation reactions forming different mono- and di-conjugates at the 4'- and/or 7-position. The kinetic constants for glucuronidation of genistein were obtained from in vitro incubations by Islam et al. [12]. For other reactions including sulfation of genistein, G-7G, and G-4'G as well as glucuronidation of G-7G, G-4'G, and G-7S the kinetic constants were obtained in the present study based on in vitro experiments. The apparent $V_{\max }$ values for formation of the different mono- and di-conjugates were scaled to in vivo $V_{\max }$ values using a $\mathrm{S} 9$ content of 11.4 and $143 \mathrm{mg} / \mathrm{g}$ tissue for small intestine and liver as scaling factors [29]. It is of importance to note that the current PBK model does not include the formation of the microbiota metabolite equol, a potent estrogenic metabolite [34, 35], because equol is a major metabolite of daidzein, not of genistein and only approximately one-third to one-half of the population are actually equol producers [36]. Dihydrogenistein is a corresponding metabolite formed of genistein by gut microbiota, however only a few studies have been reported on its estrogenicity [34, 35]. Morito et al. [34] reported that dihydrogenistein showed similar binding affinity and induced similar transcriptional activation of human ER $\alpha$ and ER $\beta$ as genistein aglycone. Contradictory, Hwang et al. [35] found that dihydrogenistein exerted much weaker estrogenic responses in binding and inducing transcriptional activation of human ER $\alpha$ and ER $\beta$ as well as in stimulating ER $\alpha$ mediated cell proliferation than genistein aglycone. Given these contradicting reports on estrogenicity formation of the microbiota metabolite dihydrogenistein was not included in the PBK model.

The physiological parameters including organ volumes, cardiac output and blood flows were obtained from Brown et al. [37] are shown in Table S4.1 in the Supporting information. The physico-chemical parameters for genistein and its metabolites are shown in Table 4.1. Tissue:blood partition coefficients of genistein and its metabolites were estimated from $\log K_{\text {ow }}$ according to a method described by DeJongh et al. [38]. Log $K_{\text {ow }}$ values were 
estimated from Clog $P$ values obtained from Scifinder (American Chemical Society, USA) and ChemBioDraw Ultra 14.0 (Cambridge-Soft, USA). Conversion of blood concentrations of genistein and its metabolites to plasma concentrations was done by dividing the blood concentrations by a blood/plasma ratio $(R)$. The $R$ value of 0.66 was used and calculated using Simcyp prediction tools [39, 40]. Mass balance equations were numerically integrated in Berkeley Madonna version 8.3.18 (Macey and Oster, UC Berkeley, CA) using Rosenbrock's algorithm for stiff system. Mass balance equations for genistein and its metabolites were similar to those previously described by Boonpawa et al. for quercetin [29, 30]. Model performance was evaluated by comparing the predicted amounts of genistein metabolites excreted in urine to the reported amounts of genistein metabolites in human urine $[17,18]$. A sensitivity analysis was performed to assess key parameters that contribute substantially to the $\mathrm{C}_{\max }$ of genistein aglycone in plasma following intake of genistein and genistin at oral doses of 0.5 and $7 \mathrm{mg} / \mathrm{kg}$ bw, representing an average Asian dietary [1] and supplementary intake of genistein/genistin [11], respectively. Normalized sensitivity coefficients (SCs) were calculated for the model parameters according to the method described by Evens and Andersen as follows [41]:

$$
\mathrm{SC}=\left(\mathrm{C}^{\prime}-\mathrm{C}\right) /\left(\mathrm{P}^{\prime}-\mathrm{P}\right) \times(\mathrm{P} / \mathrm{C})
$$

where $C$ is the initial value of the model output, $C^{\prime}$ is the modified value of the model output resulting from an increase in parameter value, $P$ is the initial parameter value, and $P^{\prime}$ is the modified parameter value assuming a $5 \%$ increase in its value.

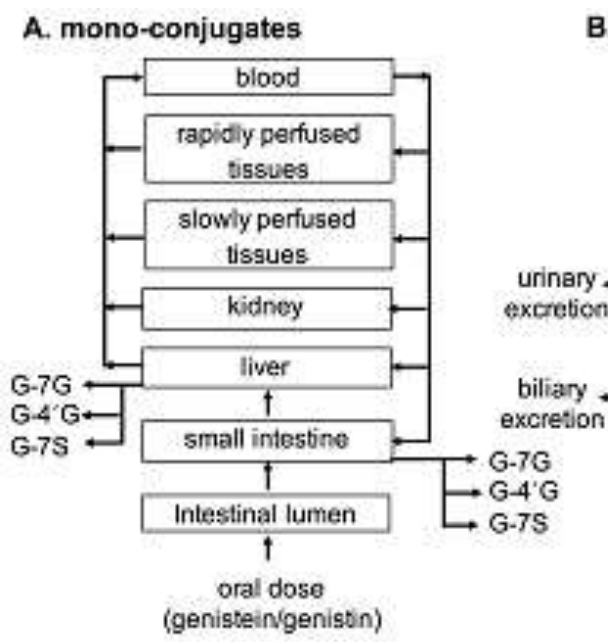

\section{B. di-conjugates}

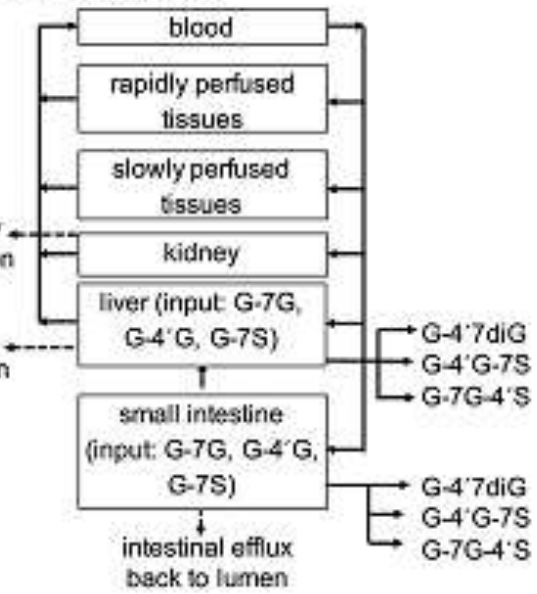

Figure 4.2. PBK model structure for mono-conjugates (A) and di-conjugates (B) of genistein: genistein-7-O-glucuronide (G-7G), genistein-4'-O-glucuronide $\left(\mathrm{G}-4^{\prime} \mathrm{G}\right)$, genistein-7-O-sulfate (G-7S), genistein-4'-O-sulfate (G-4’S), genistein-4',7-O-diglucuronide (G-47diG), genistein-7-Oglucuronide-4'-O-sulfate (G-7G-4'S), and genistein-4'-O-glucuronide-7-O-sulfate (G-4'G-7S). 
Table 4.1 Physico-chemical parameters of genistein and its metabolites in human

\begin{tabular}{|c|c|c|c|c|c|c|c|c|c|}
\hline \multirow[b]{2}{*}{ Compound } & \multirow[b]{2}{*}{$C \log P^{1}$} & \multirow[b]{2}{*}{$p \mathrm{Ka}^{2}$} & \multirow[b]{2}{*}{$\mathbf{f u}^{3}$} & \multirow[b]{2}{*}{$R^{4}$} & \multicolumn{5}{|c|}{ Tissue:blood partition coefficients ${ }^{5}$} \\
\hline & & & & & $\begin{array}{c}\text { Small } \\
\text { intestine }\end{array}$ & Liver & Kidney & $\begin{array}{c}\text { Rapidly } \\
\text { perfused tissue }\end{array}$ & $\begin{array}{c}\text { Slowly } \\
\text { perfused tissue }\end{array}$ \\
\hline Genistein & 3.11 & 6.51 & 0.03 & 0.73 & 6.44 & 6.44 & 3.01 & 3.01 & 4.08 \\
\hline G-7G & 0.84 & 2.73 & 0.16 & 0.64 & 0.86 & 0.86 & 0.92 & 0.92 & 0.90 \\
\hline G-4'G & 0.36 & 2.78 & 0.22 & 0.63 & 0.71 & 0.71 & 0.84 & 0.84 & 0.81 \\
\hline G-7S & 2.51 & -4.92 & 0.05 & 0.70 & 4.18 & 4.18 & 1.97 & 1.97 & 2.79 \\
\hline G-4'S & 2.03 & -4.24 & 0.07 & 0.68 & 2.56 & 2.56 & 1.46 & 1.46 & 1.87 \\
\hline G-7G-4'S & -1.69 & - & - & - & 0.59 & 0.59 & 0.76 & 0.76 & 0.75 \\
\hline G-4'G-7S & -1.69 & - & - & - & 0.59 & 0.59 & 0.76 & 0.76 & 0.75 \\
\hline G-4'7diG & -1.91 & 2.73 & 0.66 & 0.57 & 0.59 & 0.59 & 0.76 & 0.76 & 0.75 \\
\hline $\begin{array}{l}{ }^{1} \text { CLog } P \text { value } \\
\text { ChemBioDrav } \\
{ }^{2} p \text { Ka values } \\
{ }^{3} \text { Fraction unb } \\
{ }^{4} \text { Blood/plasm } \\
{ }^{5} \text { Tissue:blood } \\
\text { DeJongh et al. }\end{array}$ & $\begin{array}{l}\text { or genist } \\
\text { ltra } 14.0 \\
\text { obtaine } \\
\text { d (fu) w } \\
\text { atio }(R) \\
\text { rtition co } \\
\text { 8]. }\end{array}$ & $\begin{array}{l}\text { and it } \\
\text { ambric } \\
\text { rom Sc } \\
\text { calcula } \\
\text { calcul } \\
\text { icients }\end{array}$ & $\begin{array}{l}\text { esoft, } \\
\text { nder } \\
\text { d usir } \\
\text { d us } \\
\text { geni }\end{array}$ & $\begin{array}{l}\text { lites w } \\
\text { USA). } \\
\text { Ameri } \\
\text { g Simc } \\
\text { ag Sim } \\
\text { tein an }\end{array}$ & $\begin{array}{l}\text { re estimat } \\
\text { in Chemic } \\
\text { p predictic } \\
\text { ip predict } \\
\text { its metab }\end{array}$ & $\begin{array}{l}\text { Societ } \\
\text { ools-f } \\
\text { tools- } \\
\text { es we }\end{array}$ & $\begin{array}{l}\text { nder (A } \\
\text { SA). } \\
\text { ]. } \\
\text { d to pla } \\
\text { lculated }\end{array}$ & $\begin{array}{l}\text { partition ratio } \\
\text { ording to the me }\end{array}$ & od described by \\
\hline
\end{tabular}

\subsubsection{Translation of in vitro estrogenic activity to in vivo estrogenicity}

PBK model based reverse dosimetry was carried out to predict in vivo estrogenic responses upon exposure to genistein and genistin by relating the in vitro effect to plasma concentrations of genistein aglycone based on the methods described by Louisse et al. [42] and Strikwold et al. [43]. We considered the plasma $\mathrm{C}_{\max }$ as relevant parameter for these conversions to predict dose dependent in vivo estrogenic responses to genistein or genistin, because interaction of phytoestrogens with ERs is a short term effect. Furthermore, in vivo estrogenicity of genistein and genistin will especially depend on plasma concentrations of the free aglycone since its phase II metabolites have been shown to exert no estrogenic response in ER $\alpha$-positive MCF-7 and T47D breast cancer cells [13, 19]. Even though genistein monoglucuronides were reported to moderately bind to recombinant human ER $\alpha$ and ER $\beta$, this binding appears not to result in efficient transcriptional activation of both ERs as shown in yeast cells carrying human ERs [6]. For translation, available concentration-response curves for in vitro estrogenic activities of genistein available in literature were used, including concentration-response curves for 1) binding of genistein to human $\operatorname{ER} \alpha$ and $\operatorname{ER} \beta$ [4-8], 2) genistein induced $\mathrm{ER} \alpha$ and $\mathrm{ER} \beta$ mediated gene expression in U2OS reporter gene assays [10, 19, 44, 45], and 3) genistein stimulated cell proliferation in ER $\alpha$-positive MCF-7 and T47D breast cancer cells [5-10, 13, 19, 46-50]. Before making the in vitro to in vivo translation, it is of importance to realize that only the free fraction of the chemical will exert the effects which 
implies a correction is required for the binding of genistein to protein and lipid. This correction was made to take into account differences in albumin and lipid concentrations between in vitro and in vivo conditions. The binding of genistein to human ER $\alpha$ and ER $\beta$ were obtained from in vitro assays containing no protein and lipid, thus assumed 100\% availability in vitro. In this case each nominal in vitro effect concentration $\left(\mathrm{EC}_{\text {in vitro }}\right)$ of genistein aglycone for the binding activity was extrapolated to an in vivo effect concentration

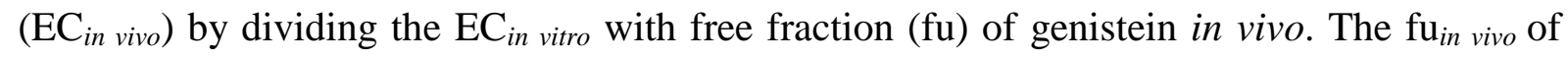
genistein in human plasma was estimated to be 0.03 (see Table 4.1). For other estrogenic responses, including genistein induced ER $\alpha$ and ER $\beta$ mediated gene expression and stimulated cell proliferation, each $\mathrm{EC}_{\text {in vitro }}$ of genistein aglycone was extrapolated to an $\mathrm{EC}_{\text {in }}$ vivo according to the extrapolation rule of Gülden and Seibert as follows [51].

$$
\mathrm{EC}_{\text {in vivo }}=\mathrm{EC}_{\text {in vitro }} \mathrm{x}\left[\left(1-\mathrm{fb}_{\text {in vitro }}\right) \times \frac{1+\mathrm{K}_{\mathrm{OW}} \times \mathrm{V}_{\mathrm{L}, \text { serume }}^{\prime}}{1+\mathrm{K}_{\mathrm{OW}} \times \mathrm{V}_{\mathrm{L}, \text { in vitro }}}+\mathrm{fb}_{\text {in vitro }} \mathrm{x} \frac{\mathrm{P}_{\text {serum }}}{\mathrm{P}_{\text {in vitro }}}\right]
$$

where $\mathrm{fb}_{\text {in vitro }}$ is the fraction of chemical bound to protein and lipid in culture medium, which is calculated from $1-\mathrm{fu}_{\text {in vitro }} . \mathrm{V}_{\mathrm{L}}^{\prime}$ is the lipid fraction in serum and in vitro. $\mathrm{P}$ is the protein content in plasma serum and culture medium.

For the extrapolation, in vivo plasma albumin and lipid concentrations of $600 \mu \mathrm{M}$ and $6 \mathrm{~g} / \mathrm{l}$, were used [51]. The albumin and lipid concentrations in culture medium of the reported in vitro bioassays were estimated from the reported data by Gülden et al. [52] indicating that $5 \%$ FBS contains $18 \mu \mathrm{M}$ BSA and $0.1 \mathrm{~g} / \mathrm{l}$ lipid. The $\mathrm{fu}_{\text {in vitro }}$ of genistein in in vitro medium was estimated using the reported binding constant of genistein to BSA of $1.3 \times 10^{4} \mathrm{M}^{-1}$ and a binding site number of 0.9 [53]. The $\mathrm{EC}_{\text {in vivo }}$ obtained was set equal to the plasma $\mathrm{C}_{\max }$ of genistein aglycone in the PBK model. The PBK model was subsequently used to calculate the corresponding oral dose levels in humans. This provided a dose-response curve that was subsequently used to derive the benchmark dose at $5 \%$ response $\left(\mathrm{BMD}_{05}\right)$ based on the default benchmark response for continuous data [54]. The $\mathrm{BMD}_{05}$ values were estimated by fitting to the Hill model, using Benchmark Dose Software version 2.6 (The Environmental Protection Agency's, USA). The $\mathrm{BMD}_{05}$ values obtained for estrogenic responses of genistein were evaluated against reported active [20-23] and inactive [2, 24-26] dose levels of genistein on estrogenicity in humans as taken from the literature and summarized in Table 4.2. 


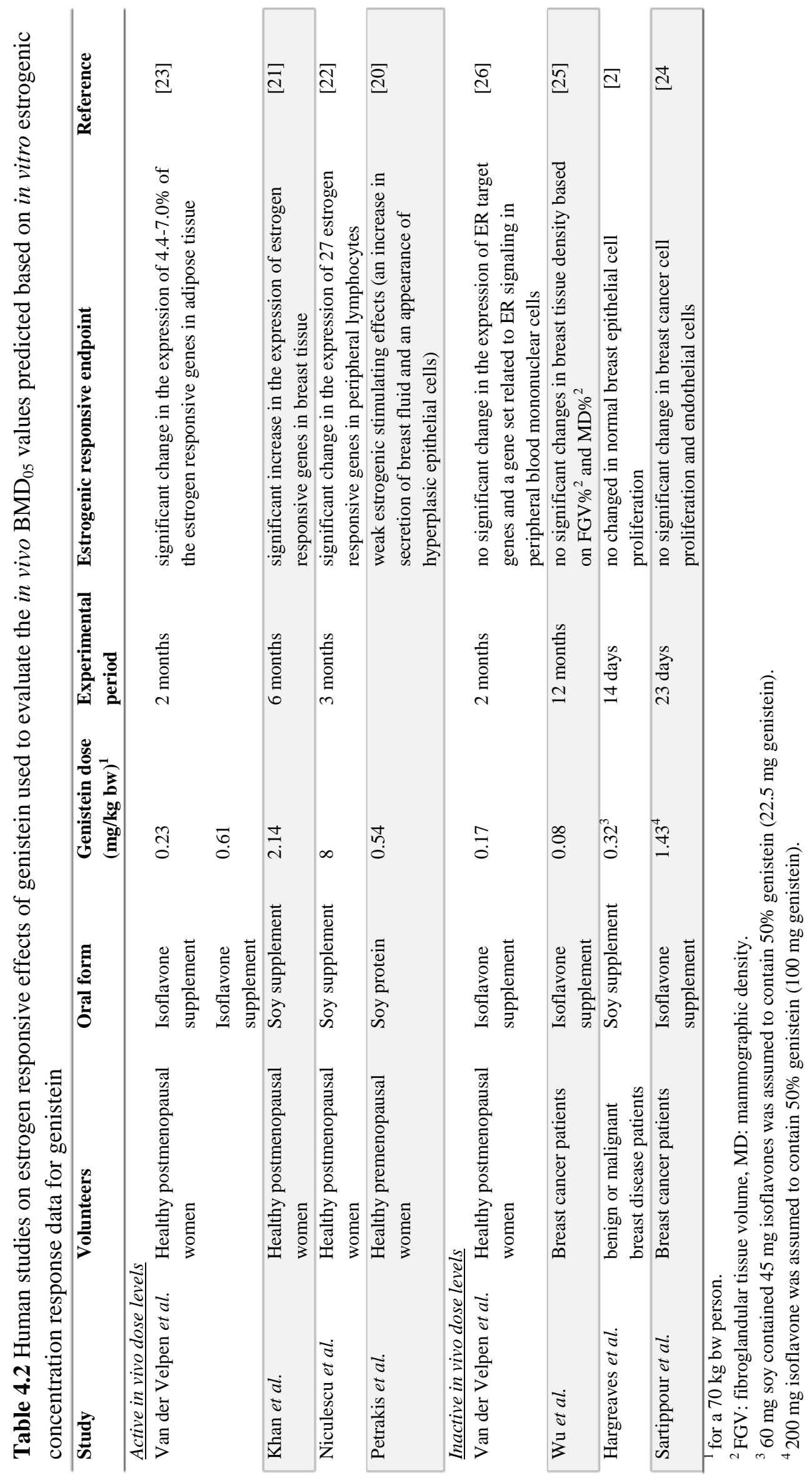




\subsection{Results}

4.3.1 Enzymatic conjugation of genistein and genistein mono-conjugates in incubations with pooled human tissue fractions

Analysis of the glucuronidation of G-7G, G-4' G, and G-7S in incubations with pooled human tissue fractions revealed that all three substrates were metabolized by pooled human small intestine S9, while pooled human liver S9 only metabolized G-4' G. The metabolite formed in incubations with G-7G and G-4' G coeluted with and showed similar spectral characteristics as commercially available G-4'7diG and was thus identified as G-4'7diG. The metabolite formed in glucuronidation incubations with G-7S with a retention time of 2 min was identified by treating the unterminated samples with $\beta$-glucuronidase/sulfatase resulting in a complete disappearance of the respective peak and a concomitant equivalent increase in the peak area of genistein aglycone (data not shown), confirming it to be a glucuronidated conjugate of G-7S. This metabolite was most likely G-7S-4' G because conjugation of flavonoids likely occurs at free $\mathrm{OH}$ positions [55].

Analysis of the sulfation incubations, revealed that human small intestine and liver S9 could metabolize genistein and G-7G, whereas sulfation of G-4' G was only observed in incubations with human liver S9. At the specified conditions in the present study, only one sulfated metabolite was formed in the incubations with genistein. The formed metabolite coeluted with and showed similar spectral characteristics as commercially available G-7S and was thus identified as G-7S. One metabolite was formed in the sulfation incubations with G7G. The formed metabolite coeluted with and showed similar spectral characteristics as commercially available G-7G-4' S and was thus identified as G-7G-4' S. The metabolite formed in sulfation incubations with $\mathrm{G}-4^{\prime} \mathrm{G}$ with a retention time of 2 min was identified by treating the unterminated samples with $\beta$-glucuronidase/sulfatase resulting in a complete disappearance of the respective peak and a concomitant equivalent increase in the peak area of genistein aglycone (data not shown), confirming it to be a sulfated conjugate of G-4' G. This metabolite was most likely G-4'G-7S because conjugation of flavonoid likely occurs at free $\mathrm{OH}$ positions [55].

\subsubsection{Kinetic constants for formation of genistein mono- and di-conjugates}

The concentration-dependent rates of glucuronidation and sulfation of genistein, G7G, G-4' G, and G-7S by human small intestine and liver S9 fractions are shown in the Supporting information (Figure S4.1). The kinetic constants for formation of all metabolites 


\section{Chapter 4}

derived from these results are summarized in Table 4.3. The results showed that the total catalytic efficiency for metabolic conversion of genistein amounted to $2.34 \mathrm{l} / \mathrm{h} / \mathrm{g}$ tissue in liver which was 7.5 -fold higher than the total catalytic efficiency of $0.31 \mathrm{l} / \mathrm{h} / \mathrm{g}$ tissue for small intestine. Genistein mono-conjugates especially G-7G and G-7S were the major metabolites formed in incubations with both small intestine and liver S9. Formation of G-7S by human liver S9 showed a strong substrate inhibition at concentration above $1 \mu \mathrm{M}$ with a $K_{\mathrm{i}}$ value of $25 \mu \mathrm{M}$. Formation of genistein di-conjugates was more than 10-fold less efficient than formation of its respective mono-conjugates due to a decrease in the $V_{\max }$ and an increase in $K_{\mathrm{m}}$ values.

Table 4.3 Kinetic constants for formation of mono- and di-conjugates of genistein obtained from in vitro incubations with human tissue fractions. For full concentration dependent activity curves from which these kinetic constants are derived (see Figure S4.1 in the Supporting information).

\begin{tabular}{|c|c|c|c|c|c|c|c|c|c|}
\hline \multirow[t]{3}{*}{ Substrate } & \multirow[t]{3}{*}{ Metabolites } & \multicolumn{4}{|c|}{ Small intestine S9 } & \multicolumn{4}{|c|}{ Liver S9 } \\
\hline & & $K_{\mathrm{m}}{ }^{1}$ & $V_{\max }^{2}$ & Scaled & $\mathrm{CE}^{4}$ & $K_{\mathrm{m}}{ }^{1}$ & $V_{\max }^{2}$ & Scaled & $\mathrm{CE}^{4}$ \\
\hline & & & & $V_{\max }^{3}$ & & & & $V_{\max }^{3}$ & \\
\hline \multirow[t]{4}{*}{ Genistein } & G-7G & 5.8 & 0.7 & 0.48 & 0.08 & 22 & 1.50 & 12.87 & 0.59 \\
\hline & $G-4^{\prime} G^{5}$ & 19 & 0.2 & 0.14 & 0.01 & 32 & 0.10 & 0.86 & 0.03 \\
\hline & $\mathrm{G}_{7} \mathrm{~S}^{6}$ & 0.11 & 0.03 & 0.02 & 0.19 & 0.11 & 0.02 & 0.17 & 1.56 \\
\hline & G4'S & $\mathrm{nd}^{7}$ & nd & - & & nd & nd & - & - \\
\hline \multirow[t]{2}{*}{ G-7G } & G-4',7diG & 229 & 0.04 & 0.03 & 0.0001 & nd & nd & - & - \\
\hline & G-7G-4'S & 134 & 0.003 & 0.002 & 0.00002 & 35 & 0.002 & 0.02 & 0.0005 \\
\hline \multirow[t]{2}{*}{ G-4'G } & G-4',7diG & 665 & 0.51 & 0.35 & 0.001 & 196 & 0.01 & 0.09 & 0.0004 \\
\hline & G-4'G-7S & nd & nd & - & - & 152 & 0.02 & 0.17 & 0.001 \\
\hline G-7S & G-7S-4'G & 18 & 0.65 & 0.44 & 0.02 & nd & nd & - & - \\
\hline \multicolumn{10}{|l|}{${ }^{1} \mu \mathrm{M}}$. \\
\hline \multicolumn{10}{|c|}{$2 \mathrm{nmol} / \mathrm{min} / \mathrm{mg} \mathrm{S} 9}$. \\
\hline \multicolumn{10}{|c|}{${ }^{3}$ Expressed as $\mu \mathrm{mol} / \mathrm{h} / \mathrm{g}$ tissue and calculated from $V_{\max (\mathrm{app})} /(1000 \mathrm{nmol} / \mu \mathrm{mol}) \times(60 \mathrm{~min} / \mathrm{h}) \times(\mathrm{mg} \mathrm{S} 9 / \mathrm{g}$ tissue $)$} \\
\hline \multicolumn{10}{|c|}{ S9 protein content yield 11.4 and $143 \mathrm{mg} / \mathrm{g}$ tissue for small intestine and liver, respectively. } \\
\hline \multicolumn{10}{|c|}{${ }^{4}$ Catalytic efficiency expressed as $1 / \mathrm{h} / \mathrm{g}$ tissue. } \\
\hline \multicolumn{10}{|c|}{${ }^{5}$ Kinetic constants for formation of genistein monoglucuronides were obtained from Islam et al. [12]. } \\
\hline \multicolumn{10}{|c|}{${ }^{6}$ Human liver S9 showed G-7S substrate inhibition at concentration $>1 \mathrm{uM} ; K \mathrm{i}=25 \mu \mathrm{M}$. } \\
\hline
\end{tabular}

\subsubsection{PBK model development and evaluation}

To define the PBK model for genistein and its glycoside genistin, kinetic parameters for metabolic conversion of genistein to mono- and di-conjugates were obtained from in vitro incubations. Kinetic parameters for excretion via bile, urine, and intestinal efflux back to intestinal lumen were obtained by fitting the predicted total genistein in plasma with reported data in humans [16-18, 32, 33], resulting in an average value of $4 \mathrm{~h}^{-1}, 2 \mathrm{~h}^{-1}$, and $0.1 \mathrm{l} / \mathrm{h}$, 
respectively. Subsequently, the model thus defined was used to predict the plasma kinetics of genistein aglycone and its metabolites. The model predicted plasma $\mathrm{C}_{\max }$ and $\mathrm{AUC}$ of genistein aglycone within 2- to 4-fold deviation from the reported human plasma data (Figure 4.3A-4.3B). Furthermore, the model also adequately predicted metabolic plasma profiles of genistein metabolites within 1- to 3-fold deviation from the reported metabolic plasma profiles in humans (Figure 4.3C).

A.

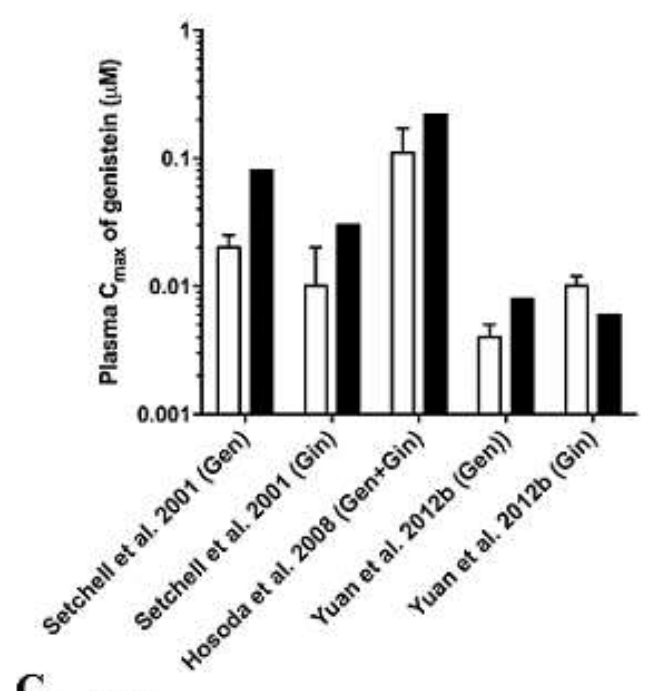

B.
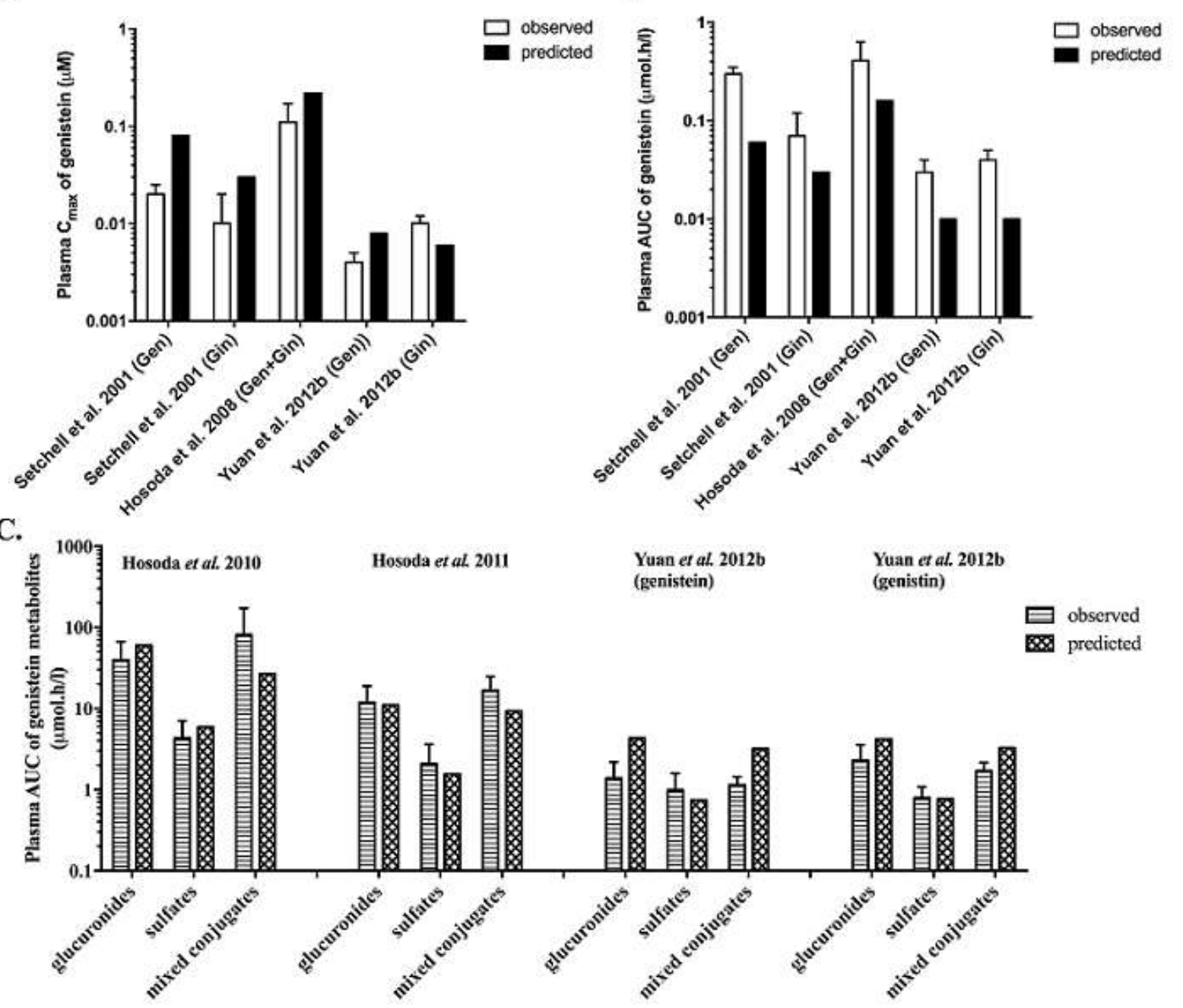

Figure 4.3 Comparison of the PBK model based predicted and experimentally reported data on (A) plasma $\mathrm{C}_{\max }$, and (B) plasma AUC of genistein aglycone, and of (C) the metabolic profile of genistein in humans [16-18, 32, 33]. The in vivo data come from Setchell et al. at a dose of $0.71 \mathrm{mg} / \mathrm{kg}$ bw genistein or $0.71 \mathrm{mg} / \mathrm{kg}$ bw genistin [33], Hosoda et al. at a dose of $0.92 \mathrm{mg} / \mathrm{kg}$ bw genistein and 0.86 $\mathrm{mg} / \mathrm{kg}$ bw genistin [32], Hosoda et al. at a dose of $0.92 \mathrm{mg} / \mathrm{kg}$ bw genistein and $0.76 \mathrm{mg} / \mathrm{kg}$ bw genistin [16], Hosoda et al. at a dose of $0.18 \mathrm{mg} / \mathrm{kg}$ bw genistein and $0.15 \mathrm{mg} / \mathrm{kg}$ bw genistin [17], and Yuan et al. at a dose of $0.13 \mathrm{mg} / \mathrm{kg}$ bw genistein or $0.21 \mathrm{mg} / \mathrm{kg}$ bw genistin [18]: Gen = genistein, Gin $=$ genistin, glucuronides $=$ sum of genistein mono- and diglucuronides, sulfates $=$ sum of genistein monosulfates, and mixed conjugates = sum of genistein metabolites containing glucuronide and sulfate moieties. 
The performance of the newly defined PBK model was evaluated by comparing the predicted amounts of genistein metabolites excreted in urine against reported data in humans for excretion of genistein metabolites in urine $[17,18]$. The results revealed that the model adequately predicted the amount of genistein metabolites excreted in urine within 2- to7-fold deviation from the reported urinary profiles of genistein metabolites in humans $[17,18]$ (Figure 4.4). These results also indicate that the model adequately predicted the kinetics of genistein in humans.

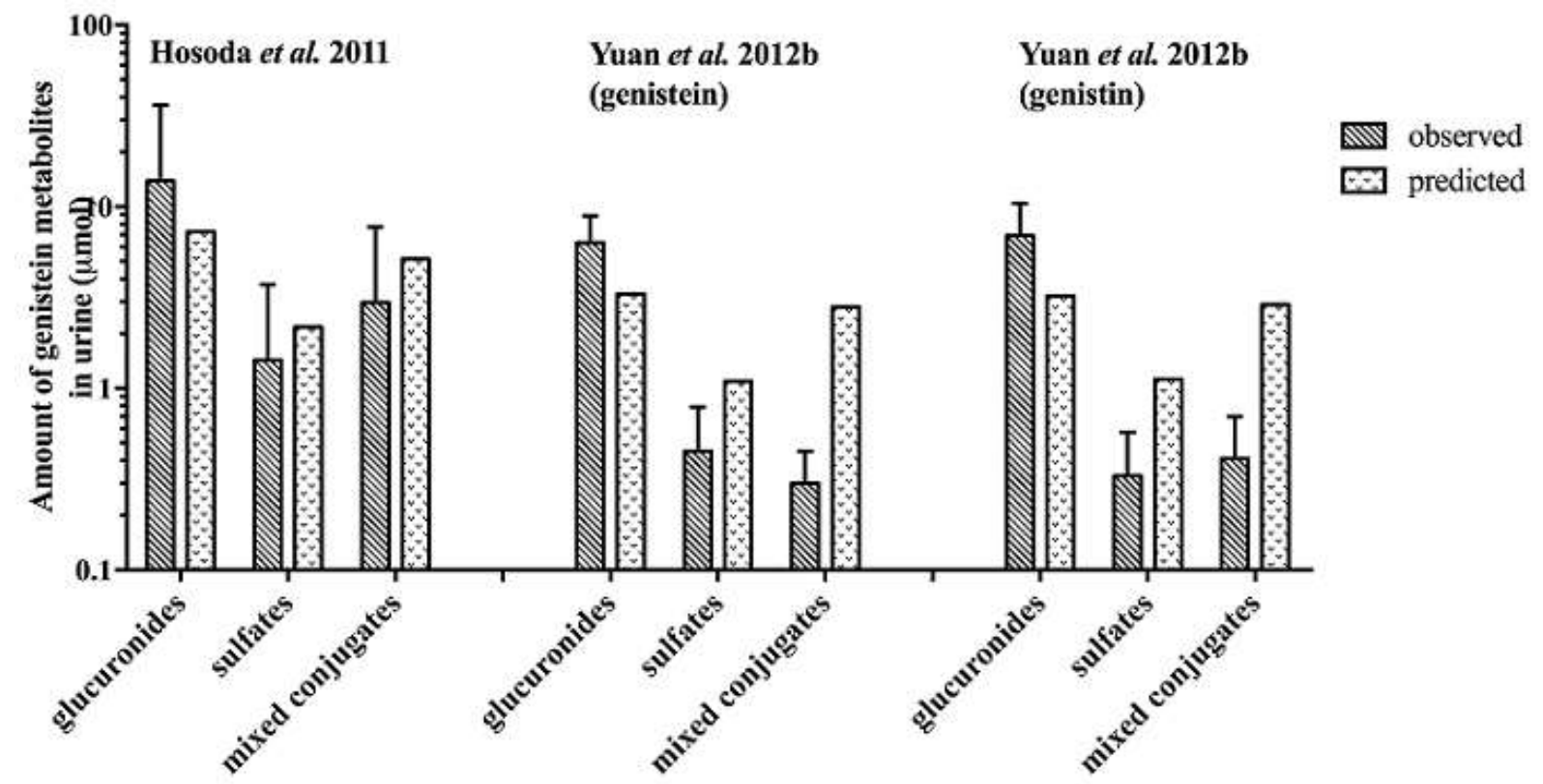

Figure 4.4 Model evaluation by comparison of the predicted urinary profiles of genistein metabolites against urinary profiles of genistein metabolites in humans as reported by Hosoda et al. at a dose of $0.18 \mathrm{mg} / \mathrm{kg}$ bw genistein and $0.15 \mathrm{mg} / \mathrm{kg}$ bw genistin [17], and by Yuan et al. at a dose of $0.13 \mathrm{mg} / \mathrm{kg}$ bw genistein or $0.21 \mathrm{mg} / \mathrm{kg}$ bw genistin [18]: Gen = genistein, Gin = genistin, glucuronides = sum of genistein mono- and diglucuronides, sulfates $=$ sum of genistein monosulfates, and mixed conjugates $=$ sum of genistein metabolites containing glucuronide and sulfate moieties.

To further evaluate the performance of the newly defined model, a sensitivity analysis was performed to assess key parameters that influence the predicted plasma $\mathrm{C}_{\max }$ of genistein aglycone at oral doses of 0.5 and $7 \mathrm{mg} / \mathrm{kg}$ bw genistein/genistin (expressed in genistein equivalents), representing an average Asian dietary [1] and a supplementary intake of genistein/genistin [11]. Only parameters that had a normalized sensitivity coefficient higher than 0.1 (in absolute value) are displayed in Figure S4.2 in the Supporting information. When consumed as aglycone, the most influential physiological and physico-chemical parameters for the predicted plasma $\mathrm{C}_{\max }$ of genistein aglycone are the body weight, the volumes of the small intestine and liver, the cardiac output, the reginal blood flow through the small intestine and rapidly perfused tissues, the small intestine tissue:blood partition coefficient of genistein, 
the uptake rate constant, and the blood/plasma ratio (expressing normalized sensitivity coefficients between 0.3 to 1.0 in absolute value). The predicted plasma $\mathrm{C}_{\max }$ of genistein aglycone is also largely affected by the parameters that influence the formation of $\mathrm{G}-7 \mathrm{G}$ and G-7S including the S9 protein yield in the small intestine and liver, and the kinetic constants for formation of G-7G and G-7S in the small intestine and liver (expressing normalized sensitivity coefficients between 0.2 to 1.20 in absolute value). As the dose increases, the kinetic parameters for formation of $\mathrm{G}-7 \mathrm{G}$ and $\mathrm{G}-7 \mathrm{~S}$ in the small intestine become less influential and the kinetics for formation of $\mathrm{G}-7 \mathrm{G}$ in the liver become the most influential kinetic parameters at high dose. When consumed as its glycoside genistin, the predicted plasma $\mathrm{C}_{\max }$ of genistein aglycone are influenced by similar parameters and in a similar manner with as additional influential parameters including the volume of the intestinal lumen and the hydrolytic clearance of genistin (expressing normalized sensitivity coefficients of approximately 0.4 in absolute value).

\subsubsection{PBK model predictions of metabolic plasma profiles of genistein}

The PBK model was used to simulate the dose-dependent plasma concentration of genistein and its metabolites at different oral dose levels. The results obtained revealed that G-7G was predicted to be the major circulating metabolite of genistein with a plasma $C_{\max }$ of 0.03-343 $\mu \mathrm{M}$ at oral doses ranging from $0.01-50 \mathrm{mg} / \mathrm{kg}$ bw genistein, contributing to $40-82 \%$ of the total plasma genistein concentrations (genistein plus metabolites) (Figure 4.5A). G-7S and G-4'G-7S were predicted to be the second and the third most prevalent circulating metabolites of genistein with a plasma $\mathrm{C}_{\max }$ of 3.2-0.03 and 8.9-0.01 $\mu \mathrm{M}$ accounting for 350.7 and $18-1.8 \%$ of the total plasma genistein concentrations at an oral dose up to $8 \mathrm{mg} / \mathrm{kg}$ bw genistein, respectively. At the higher doses, the relative contribution of these metabolites decreases, accompanied by a relative increase in the concentration of genistein aglycone, due to the saturation of sulfation reactions at high doses, resulting in a relative higher genistein aglycone concentrations in the systemic circulation. The plasma concentrations of genistein aglycone were predicted to be $0.0003-77 \mu \mathrm{M}$ amounting to $0.5-17 \%$ of total plasma genistein concentrations at oral doses ranging from $0.01-50 \mathrm{mg} / \mathrm{kg}$ bw genistein. When consumed as the glycoside genistin, similar metabolic profiles of genistein in plasma were predicted, requiring approximately 1.3-fold higher oral dose levels (expressed in genistein equipvalents) to achieve similar plasma concentrations. This is due to the slow release of genistein aglycone 
from its glycoside genistin that limits the amount of genistein being taken up resulting in lower genistein concentrations in plasma and tissues at equimolar dose levels.
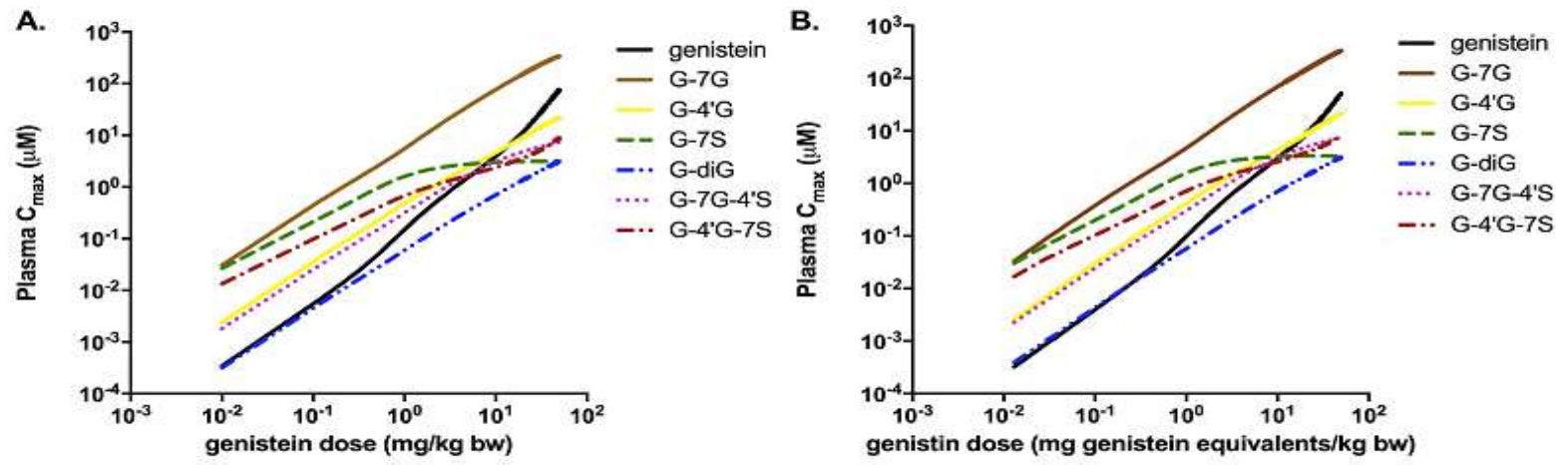

Figure 4.5 PBK model based prediction of dose-dependent plasma $\mathrm{C}_{\max }$ of genistein and its circulating metabolites at oral doses ranging from $0.01-50 \mathrm{mg} / \mathrm{kg}$ bw (A) genistein and (B) genistin (expressed in genistein equivalents).

4.3.5 Translation of in vitro concentration-response data for genistein estrogenicity to in vivo dose-response curves

Using PBK model based reverse dosimetry, the in vitro concentration-response curves for estrogenic responses of genistein were translated to in vivo dose-response curves for the binding of genistein to human $\mathrm{ER} \alpha$ and $\mathrm{ER} \beta$, genistein induced $\mathrm{ER} \alpha$ and $\operatorname{ER} \beta$ mediated gene expression, and genistein stimulated cell proliferation in ER $\alpha$-positive breast cancer cells. Figure 4.6 depicts the translation of the in vitro concentration-response curves to in vivo doseresponse curves upon oral intake of genistein (for the curves obtained from these in vitro data for in vivo dose-response curves for oral intake of genistin see Figure S4.3 in the Supporting information). The steps to be taken in this in vitro to in vivo translation can be described as follows, using the binding activity of genistein to ER $\alpha$ as an example. In the first step all available in vitro data for the binding activity of genistein to human ER $\alpha$ were collected from literature [4-7]. This provides the curves presented in the left half of Figure 4.6, so Figure 4.6A for in vitro $\mathrm{ER} \alpha$ binding of genistein. Secondly, each $\mathrm{EC}_{\text {in vitro }}$ was extrapolated to an

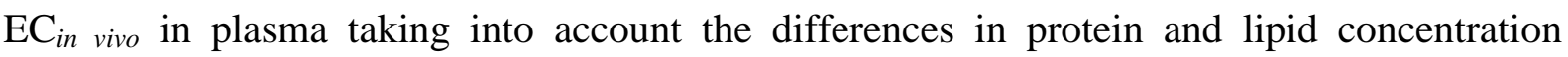
between the in vitro bioassays and the in vivo situations (see Section 4.2.6). The in vivo plasma concentrations thus obtained were then used as input in the PBK model that was used to predict the corresponding in vivo dose level. These predicted dose levels could then be used to define the in vivo dose-response curve plotting the respective dose levels against the responses detected in the in vitro bioassay. This provides the curves presented in the right half of Figure 4.6. This was followed by fitting the obtained in vivo dose-response curves to derive 
the $95 \%$ lower and upper confidence limits of the in vivo dose-response curves. The obtained in vivo dose-response curve were subsequently used to estimate the $\mathrm{BMD}_{05}$ values for estrogenicity of genistein in humans.

\section{A. ER $\alpha$ binding}
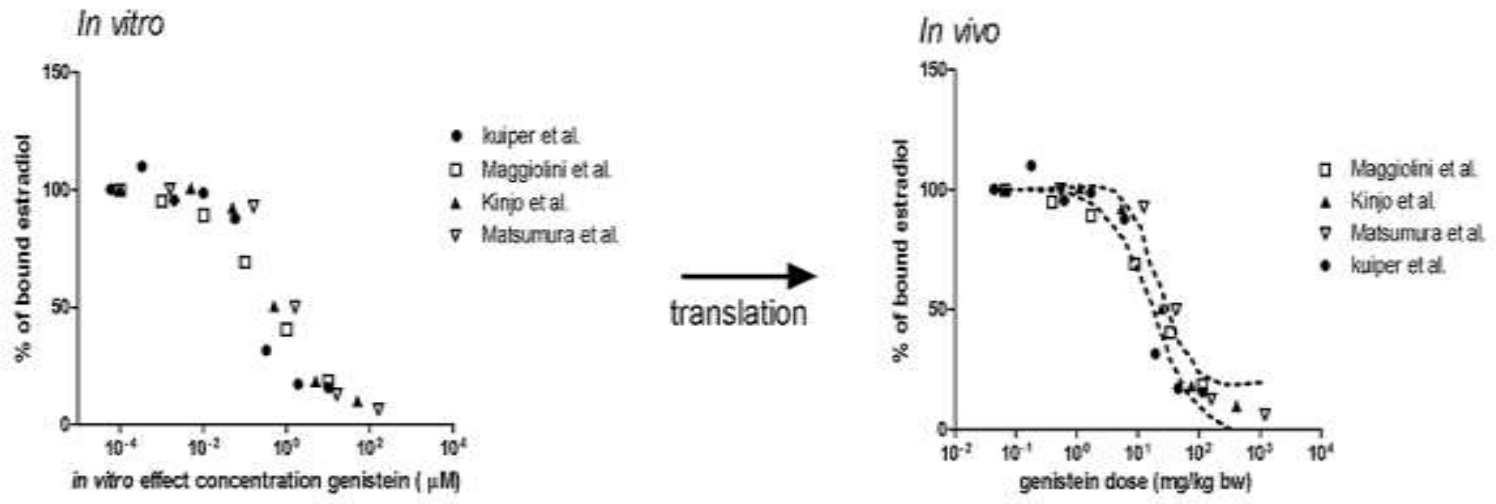

B. ER $\beta$ binding
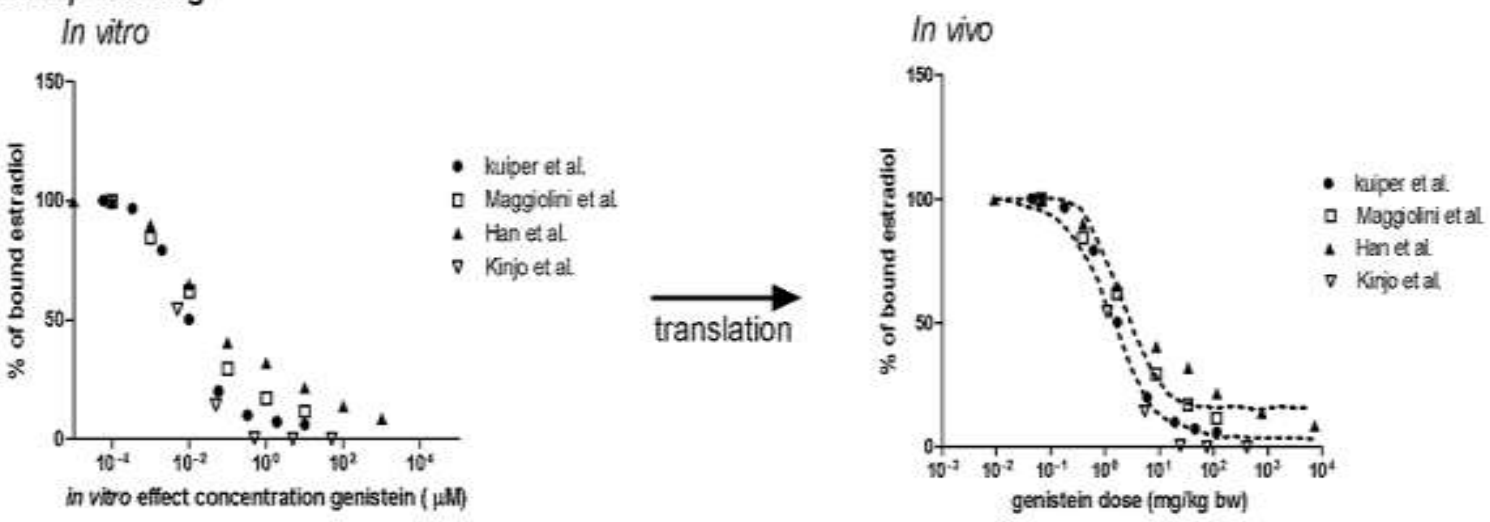

C. ER $\alpha$ transcriptive induction
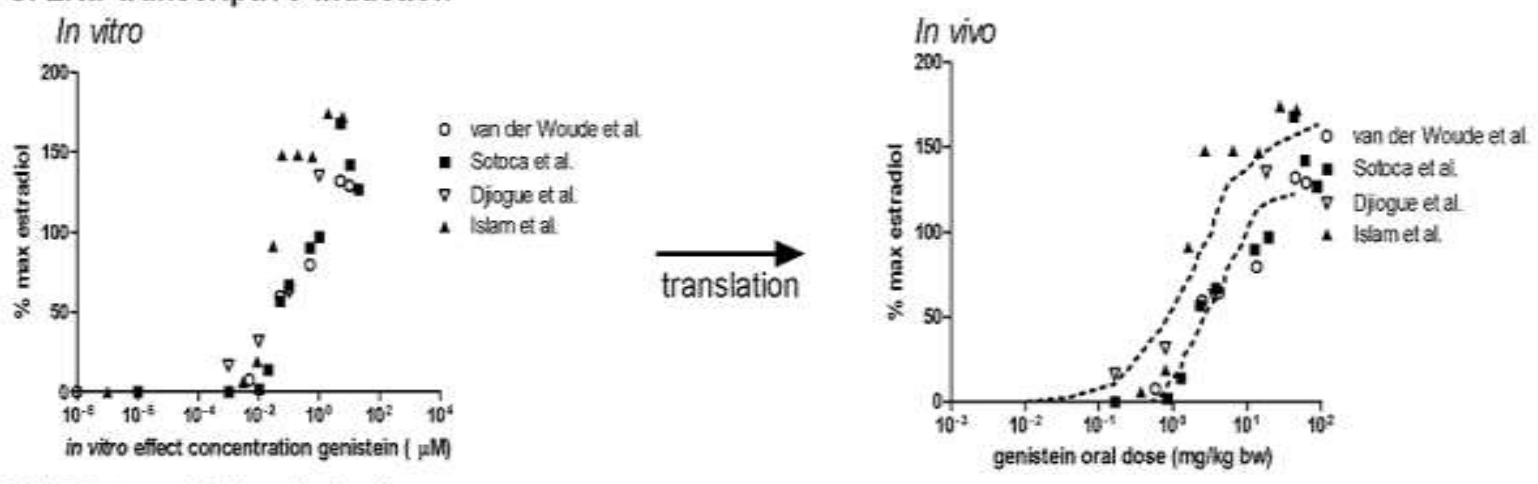

\section{ER $\beta$ transcriptive induction}
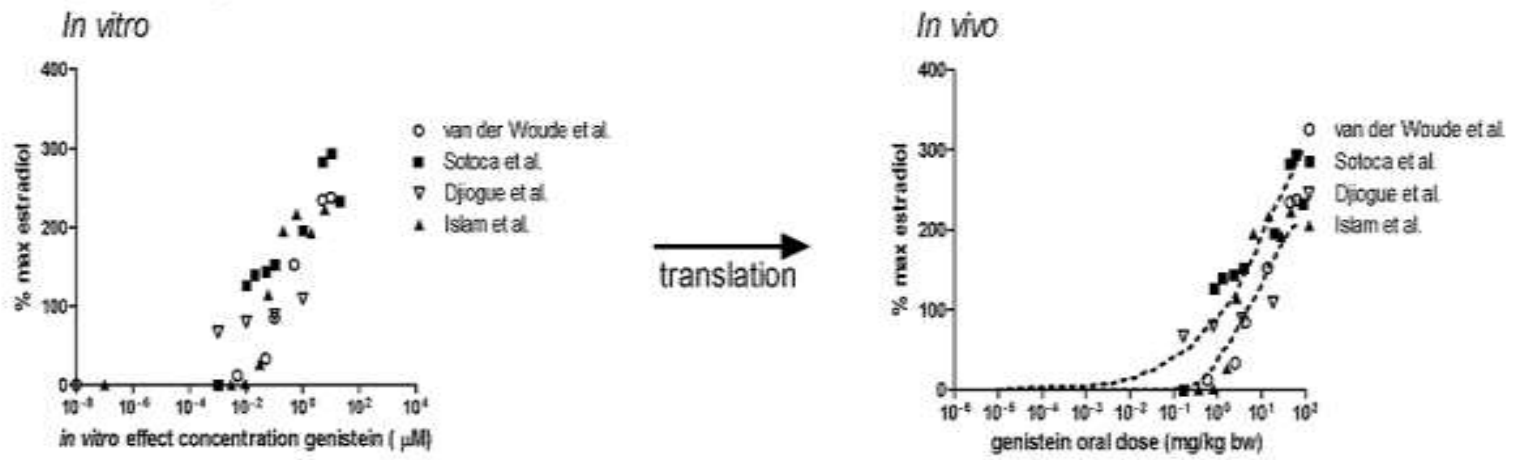
E. ER $\alpha$ dependent proliferation

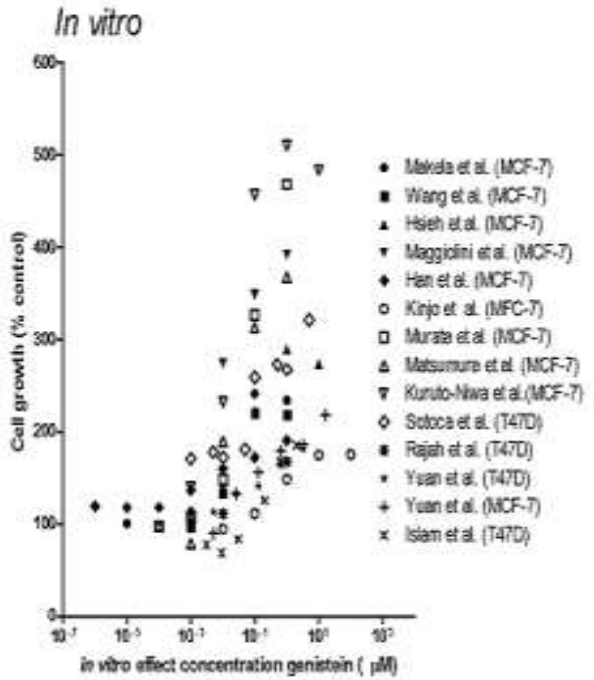

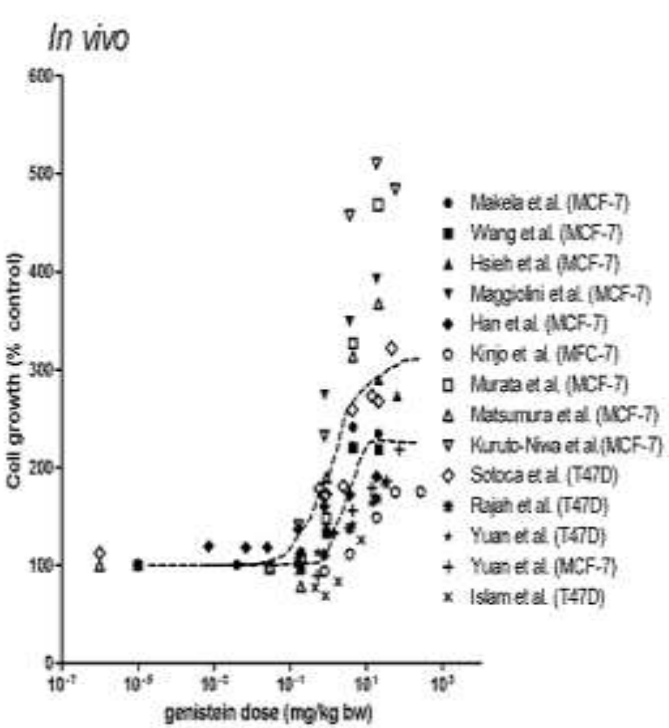

Figure 4.6 Translation of in vitro estrogenic concentration-response curves for genistein dependent (A) binding to ER $\alpha$ [4-7], (B) binding to ER $\beta$ [4-6, 8], (C) ER $\alpha$ mediated gene expression in U2OS reporter gene assays $[10,19,44,45]$, (D) ER $\beta$ mediated gene expression in U2OS reporter gene assays [10, 19, 44, 45], and (E) ER $\alpha$ dependent proliferation of MCF-7 and T47D breast cancer cells [5-10, $13,19,46-50]$, to in vivo human genistein dose-response curves using PBK model based reverse dosimetry based on plasma $\mathrm{C}_{\max }$ values of genistein aglycone. The dashed lines represent the $95 \%$ lower and upper confidence limits.

In a similar way, the $\mathrm{BMD}_{05}$ values for in vivo estrogenicity were predicted using $\mathrm{PBK}$ model based reverse dosimetry to translate in vitro estrogenic response for ER $\beta$ binding (Figure 4.6B), induction of ER $\alpha$ and ER $\beta$ mediated gene expression (Figure 4.6C and 4.6D), and ERa mediated cell proliferation (Figure 4.6E).

From the results thus obtained it could be concluded that when consumed as genistein aglycone, the in vivo $\mathrm{BMD}_{05}$ values for $\mathrm{ER} \alpha$ binding and induction of $\mathrm{ER} \alpha$ mediated gene expression based on in vitro data were predicted to occur at an oral dose ranging from 0.06$4.56 \mathrm{mg} / \mathrm{kg}$ bw genistein. The in vivo $\mathrm{BMD}_{05}$ values for $\mathrm{ER} \beta$ binding and induction of $\mathrm{ER} \beta$ mediated gene expression based on in vitro data were predicted to occur at an oral dose ranging from $0.06-0.33 \mathrm{mg} / \mathrm{kg}$ bw genistein. The in vivo $\mathrm{BMD}_{05}$ values for estrogenic response of genistein on $\mathrm{ER} \alpha$ dependent cell proliferation based on in vitro data were predicted to occur at an oral dose ranging from $0.03-0.72 \mathrm{mg} / \mathrm{kg}$ bw genistein. When consumed as the glycoside genistin, the predicted in vivo $\mathrm{BMD}_{05}$ values for estrogenicity based on in vitro data are approximately 1.3-fold higher for all in vitro estrogenic endpoints as compared with the predicted in vivo $\mathrm{BMD}_{05}$ values for consumption as genistein aglycone. This is due to the slow release of genistein aglycone from its glycoside genistin that limits the amount of genistein being taken up and overall lower genistein concentrations in the plasma. 
The predicted in vivo $\mathrm{BMD}_{05}$ values for estrogenic responses based on the in vitro PBK approach were evaluated by comparing the predicted in vivo $\mathrm{BMD}_{05}$ values with the reported active [20-23] and inactive [2, 24-26] dose levels of genistein in studies on estrogenic responses in humans (Figure 4.7). The results indicate that the predicted $\mathrm{BMD}_{05}$ values for genistein induced ER mediated gene expression are in line with reported active dose levels of genistein $(0.23-8 \mathrm{mg} / \mathrm{kg}$ bw) in in vivo studies reporting induction of $\mathrm{ER}$ mediated gene expression in humans in different tissues [21-23]. Furthermore, an oral dose level of $0.54 \mathrm{mg} / \mathrm{kg}$ bw genistein reported to induce a weak estrogenic response of genistein in humans [20] also falls in the range of the predicted dose levels for estrogenicity of genistein. The overview presented in Figure 4.7 also reflects that studies reporting the absence of effects of genistein in human in vivo studies fall in the same range as the dose levels predicted and observed to have estrogenic responses. For example, Van der Velpen et al. [26] reported no significantly change in the expression ER target genes and gene sets related to ER signaling in peripheral blood mononuclear cells in human at an oral dose of $0.17 \mathrm{mg} / \mathrm{kg}$ bw genistein, which is not in line with the predicted dose levels for this estrogenic response in the present study. This result was also opposite to the result reported by the same authors at a comparable dose regimen in a similar study population of healthy postmenopausal women when analyzing expression of estrogen responsive genes in adipose tissue representing a more likely target tissue than peripheral blood mononuclear cells [23]. Genistein at dose levels between 0.08 and $1.43 \mathrm{mg} / \mathrm{kg}$ bw appeared also to not affect breast tissue density, normal breast epithelial cell proliferation, and breast cancer or epithelial cell proliferation in breast cancer patients $[2,24,25]$, although these values all fall within the range of in vivo $\mathrm{BMD}_{05}$ values predicted to be active based on ER $\alpha$ mediated in vitro cell proliferation (0.03-0.72 $\mathrm{mg} / \mathrm{kg}$ bw genistein). The reasons underlying these discrepancies remain to be elucidated, but may be related to the endpoint or target tissue studied or the study population (i.e. healthy women versus breast cancer patients).

The PBK model-based predicted dose-response curves and in vivo $\mathrm{BMD}_{05}$ values derived from them also revealed that at a Western dietary intake of genistein amounting to $0.003-0.01 \mathrm{mg} / \mathrm{kg}$ bw [11] the plasma $\mathrm{C}_{\max }$ of genistein aglycone would generally not be high enough to induce ER mediated gene expression or activities (Figure 4.7). At an Asian dietary and a supplementary intakes of genistein amounting to $0.21-0.71$ [1] and $0.43-13$ [11] $\mathrm{mg} / \mathrm{kg}$ bw, the plasma $\mathrm{C}_{\max }$ of genistein aglycone would be high enough to exert estrogenic responses induced by both ER $\alpha$ and ER $\beta$ mediated pathways (Figure 4.7). 


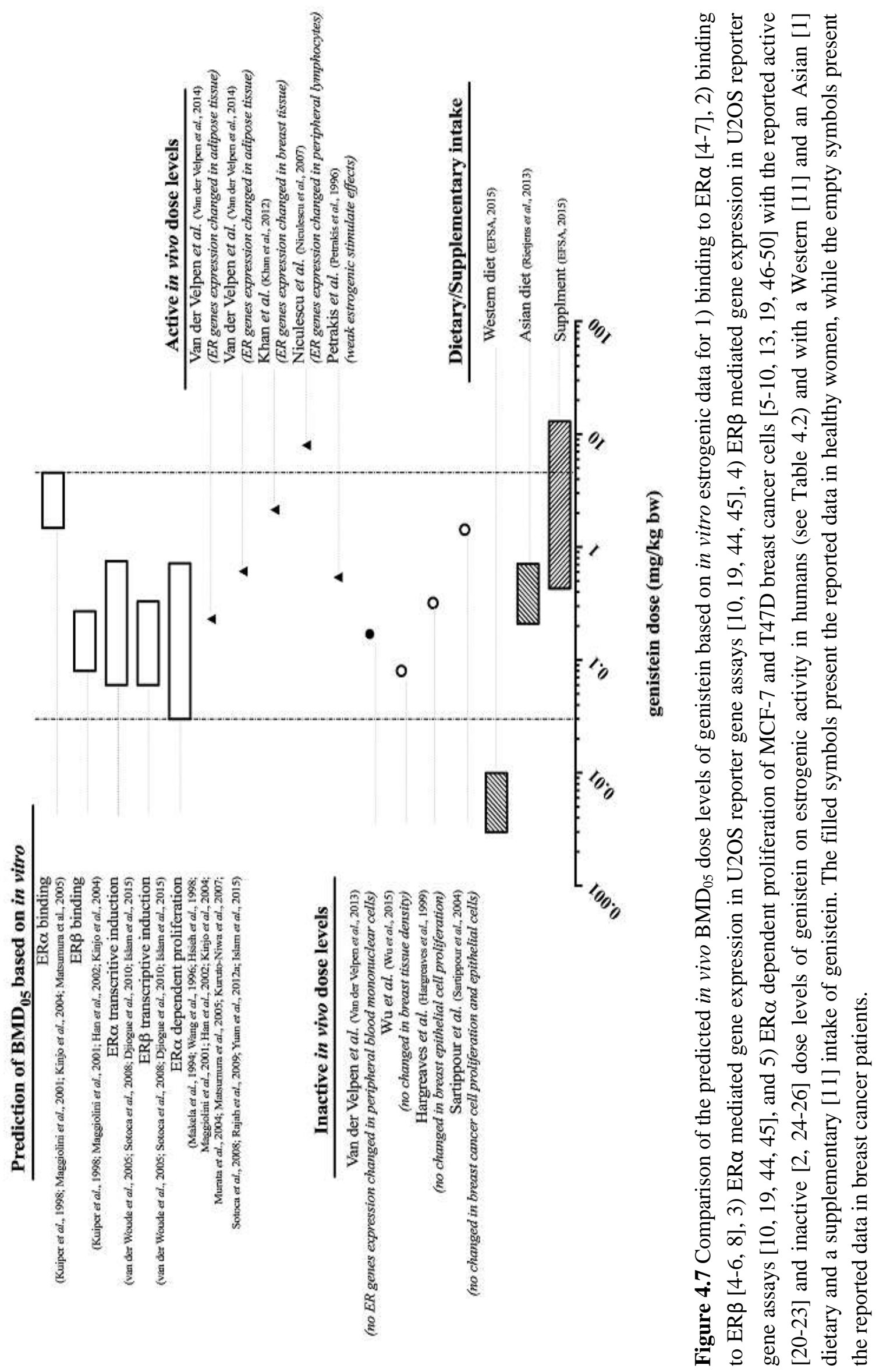




\subsection{Discussion}

The aim of the present study was to develop a PBK model that provides insight in the dose-dependent plasma concentration of genistein aglycone and its metabolites and enables prediction of the in vivo dose-response curves for estrogenicity of genistein and genistin in humans based on in vitro concentration-response curves. Evaluation of the PBK model obtained against reported data on plasma and urinary metabolic profiles of genistein revealed that the model could make adequate predictions with deviations that were limited from 1- to 7-fold. The model predicted that G-7G. was the major plasma metabolite of genistein and that plasma concentrations of free genistein were predicted to be $0.0003-77 \mu \mathrm{M}$ at oral doses ranging from $0.01-50 \mathrm{mg} / \mathrm{kg}$ bw genistein, amounting to $0.5-17 \%$ of total plasma genistein. PBK model based predictions for genistin were overall quite similar with a major difference being 1.3-fold lower plasma concentration of free genistein at similar dose levels expressed in genistein equivalents. These differences were mainly due to the slow release of genistein aglycone from its glycoside genistin that limits the amount of genistein being taken up resulting in lower genistein concentrations in the plasma.

Upon evaluation, the PBK model was used to convert available in vitro concentrationresponse curves in bioassays detecting estrogenicity of genistein to in vivo dose-response curves by reverse dosimetry, in order to better understand in vivo estrogenic responses upon intake of genistein and genistin. The in vivo dose-response curves obtained were subsequently used to derive in vivo $\mathrm{BMD}_{05}$ dose levels of genistein and genistin, at which the plasma concentration of the genistein aglycone would be high enough to induce 5\% estrogenic response. When consumed as genistein, the in vivo $\mathrm{BMD}_{05}$ values for binding and induction of ER $\alpha$ mediated gene expression based on in vitro data were predicted to be at oral doses ranging from $0.06-4.56 \mathrm{mg} / \mathrm{kg}$ bw genistein. For estrogen responses on ER $\beta$, the predicted in vivo $\mathrm{BMD}_{05}$ values based on in vitro data were at oral dose ranging from $0.06-0.33 \mathrm{mg} / \mathrm{kg} \mathrm{bw}$ genistein. The in vivo $\mathrm{BMD}_{05}$ values for ER $\alpha$ mediated cell proliferation were predicted to be at oral doses ranging from $0.03-0.72 \mathrm{mg} / \mathrm{kg}$ bw genistein. When consumed as genistin, the predicted in vivo $\mathrm{BMD}_{05}$ values were approximately 1.3-fold higher as compared to the predicted in vivo $\mathrm{BMD}_{05}$ values when consumed as genistein. This is due to the slow release of genistein aglycone from its glycoside genistin that limits the amount of genistein being taken up resulting in lower genistein concentrations in the plasma. The available in vitro data for $\mathrm{ER} \alpha$ mediated cell proliferation show large variation (Figure 4.6E), which might be due to differences between the laboratories where the cells were cultivated as highlighted by 
Chapter 4

Osborne et al. [56] who reported that even though MFC-7 cell lines obtained from different laboratories have similar karyotype, they may have unique biological properties.

Evaluation of the predicted in vivo $\mathrm{BMD}_{05}$ values obtaining from the PBK model based reverse dosimetry against the reported estrogenic responses of genistein in humans available in literature indicated that the prediction of in vivo $\mathrm{BMD}_{05}$ values based on in vitro genistein induced ER mediated estrogenicity are in line with the reported active dose levels for these estrogenic responses in humans in different tissues [21-23] (Figure 4.7). Furthermore, a dose level of $0.54 \mathrm{mg} / \mathrm{kg}$ bw genistein reported to induce weak estrogenic activity in humans also falls in the range of the predicted dose levels that exert estrogenic responses. Contradictory, some human intervention studies reported the absence of genistein induced ER mediated gene expression in peripheral blood mononuclear cells in healthy women [26] and no effect on breast tissue density, normal breast epithelial cell proliferation, and breast cancer or epithelial cell proliferation in breast cancer patients $[2,24,25]$ at the predicted oral dose levels for estrogenic responses of genistein. The reasons underlying these discrepancies remain to be elucidated, but may be related to the endpoint or target tissue studied (adipose tissue or peripheral blood mononuclear cells) or the study population (healthy woman versus breast cancer patients). The difference in the expression of ER $\alpha$ and $\mathrm{ER} \beta$ between in vitro bioassays and the target tissues studied in the breast cancer patients might result in a difference in the cellular response toward different xenoestrogens as the absolute concentration and ratio of ER $\alpha$ to ER $\beta$ within a tissue influence the cellular response toward different xenoestrogens [10]. Breast cancer cell lines like MCF-7 and T47D, present high expression of $E R \alpha$ and very low expression of $\operatorname{ER} \beta[10,57]$, reflecting what is likely for the tumors but not for healthy breast tissue [58]. Given these considerations, it would be of use that human intervention studies on the estrogenic activity of xenoestrogens should analyze and report the concentration and ratio of ER $\alpha$ and ER $\beta$ in the target tissue studied, and select a relevant surrogate tissue for the endpoint of interest in order to obtain a better accuracy of the interpretation of the estrogenic activity of the xenoestrogens in in vivo.

Based on the predicted in vivo $\mathrm{BMD}_{05}$ values for estrogenic responses of genistein derived from in vitro data, the plasma $\mathrm{C}_{\max }$ of genistein aglycone at an Asian dietary and a supplementary intake of genistein amounting to $0.21-0.71$ [1] and $0.43-13$ [11] $\mathrm{mg} / \mathrm{kg}$ bw genistein, would be high enough to induce estrogenic responses in vivo. A Western dietary intake of genistein amounting to $0.003-0.01 \mathrm{mg} / \mathrm{kg}$ bw genistein [11] would generally not be high enough to induce an estrogenic response. 
Considering that the plasma concentration of genistein aglycone could be a key parameter determining estrogenic responses in vivo, any factors that influence this parameter may influence sensitivity to estrogenic responses between individual human subjects. Based on the sensitivity analysis, the plasma concentration of genistein aglycone is highly influent by the kinetic constants for formation of G-7G in the small intestine and liver and the kinetic constants for formation of G-7S in the liver at low oral dose (at dietary intake levels). As the dose increases (at supplementary intake levels), the kinetic constants for formation of G-7G in the liver become the most influential parameters that limit the plasma concentration of genistein aglycone. This indicates a saturation of metabolism in the small intestine and a saturation of the sulfation reactions at high oral dose. This analysis suggests that especially genetic and lifestyle factors influencing the expression and/or activity of conjugating enzymes may influence the plasma concentration of genistein aglycone between individual human subjects [59]. Aside from the interindividual human variation in conjugating metabolism in the small intestine and liver, metabolism of genistein at the site of action may also play a crucial role in the bioavailability of the free aglycone at the target tissues. In the case of breast tissue, expression of $\beta$-glucuronidases (enzymes that deconjugate glucuronidated metabolites to aglycone) [19] as well as of UDP-glucuronosyl transferases [60] may influence the local concentrations of the free aglycone. The relative conjugation and deconjugation activity in breast tissue is not well studied. In the present study, the defined PBK model does not yet include such tissue specific metabolism of genistein and its metabolites in possible target tissues such as breast tissue, because there is a lack of human data to perform and evaluate this simulation. For further refinement of the predictions by the PBK model based reverse dosimetry approach for estrogenic activity of genistein, interindividual human variation in conjugating enzymes and the possible role of intracellular metabolism at the site of action could be studied to a further extent.

Overall, the present study shows that it is possible to predict dose dependent plasma concentrations of genistein and its metabolites and also at what dose levels estrogenic responses of genistein in humans can be expected, the latter by combining in vitro concentration-response curves with PBK model based reverse dosimetry, providing an in vitro-in silico approach to obtain insights in human dose-response curves without the need for human intervention studies. 
Chapter 4

\section{Conflict of interest}

The authors state no conflict of interest.

\section{Acknowledgements}

This research was financially supported by the Ministry of Science and Technology of Thailand through a Royal Thai Government Scholarship awarded to Rungnapa Boonpawa for conducting her $\mathrm{PhD}$ in The Netherlands. 
In vitro-in silico approach for genistein estrogenicity

\section{References}

1. Rietjens, I. M. C. M., et al., Mechanisms underlying the dualistic mode of action of major soy isoflavones in relation to cell proliferation and cancer risks. Molecular Nutrition \& Food Research, 2013. 57(1): p. 100-113.

2. Hargreaves, D. F., et al., Two-week dietary soy supplementation has an estrogenic effect on normal premenopausal breast. The Journal of Clinical Endocrinology \& Metabolism, 1999. 84(11): p. 4017-4024.

3. Yamamoto, S., et al., Soy, isoflavones, and breast cancer risk in Japan. Journal of the National Cancer Institute, 2003. 95(12): p. 906-913.

4. Kuiper, G. G., et al., Interaction of estrogenic chemicals and phytoestrogens with estrogen receptor beta. Endocrinology 1998. 139(10): p. 4252-4263.

5. Maggiolini, M., et al., Estrogen receptor a mediates the proliferative but not the cytotoxic dose-dependent effects of two major phytoestrogens on human breast cancer cells. Molecular Pharmacology, 2001. 60(3): p. 595-602.

6. Kinjo, J., et al., Interactions of phytoestrogens with estrogen receptors $\alpha$ and $\beta$ (III). Estrogenic activities of soy isoflavone aglycones and their metabolites isolated from human urine. Biological and Pharmaceutical Bulletin, 2004. 27(2): p. 185-188.

7. Matsumura, A., et al., Comparative study of oestrogenic properties of eight phytoestrogens in MCF7 human breast cancer cells. The Journal of Steroid Biochemistry and Molecular Biology, 2005. 94(5): p. 431-443.

8. Han, D.-H., et al., Relationship between estrogen receptor-binding and estrogenic activities of environmental estrogens and suppression by flavonoids. Bioscience, Biotechnology, and Biochemistry, 2002. 66(7): p. 1479-1487.

9. Hsieh, C.-Y., et al., Estrogenic effects of genistein on the growth of estrogen receptor-positive human breast cancer (MCF-7) cells in vitro and in vivo. Cancer Research, 1998. 58(17): p. 3833-3838.

10. Sotoca, A. M., et al., Phytoestrogen-mediated inhibition of proliferation of the human T47D breast cancer cells depends on the ER $\alpha$ ER $\beta$ ratio. The Journal of Steroid Biochemistry and Molecular Biology, 2008. 112(4-5): p. 171-178.

11. EFSA, Risk assessment for peri- and post-menopausal women taking food supplements containing isolated isoflavones. EFSA Journal 2015, 2015. 13(10): p. 4246 [page 342].

12. Islam, M. A., et al., Conversion of major soy isoflavone glucosides and aglycones in in vitro intestinal models. Molecular Nutrition \& Food Research, 2014. 58(3): p. 503-515.

13. Yuan, B., et al., Role of metabolism in the effects of genistein and its phase II conjugates on the growth of human breast cell lines. The AAPS Journal, 2012. 14(2): p. 329-344.

14. Walsh, K. R., et al., Isoflavonoid glucosides are deconjugated and absorbed in the small intestine of human subjects with ileostomies. The American Journal of Clinical Nutrition, 2007. 85(4): p. 1050-1056.

15. Bokkenheuser, V. D., C. H. Shackleton, and J. Winter, Hydrolysis of dietary flavonoid glycosides by strains of intestinal Bacteroides from humans. Biochemical Journal, 1987. 248(3): p. 953-956.

16. Hosoda, K., et al., Identification and quantification of daidzein-7-glucuronide-4'-sulfate, genistein-7-glucuronide-4'-sulfate and genistein-4',7-diglucuronide as major metabolites in human plasma after administration of kinako. Analytical and Bioanalytical Chemistry, 2010. 397(4): p. 1563-1572. 
17. Hosoda, K., T. Furuta, and K. Ishii, Metabolism and disposition of isoflavone conjugated metabolites in humans after ingestion of kinako. Drug Metabolism and Disposition, 2011. 39(9): p. 1762-1767.

18. Yuan, B., et al., Absorption and plasma disposition of genistin differ from those of genistein in healthy women. Journal of Agricultural and Food Chemistry, 2012. 60(6): p. 1428-1436.

19. Islam, M. A., et al., Deconjugation of soy isoflavone glucuronides needed for estrogenic activity. Toxicology in Vitro, 2015. 29(4): p. 706-715.

20. Petrakis, N. L., et al., Stimulatory influence of soy protein isolate on breast secretion in preand postmenopausal women. Cancer Epidemiology Biomarkers \& Prevention, 1996. 5(10): p. 785-794.

21. Khan, S. A., et al., Soy isoflavone supplementation for breast cancer risk reduction: a randomized phase II trial. Cancer Prevention Research, 2012. 5(2): p. 309-319.

22. Niculescu, M. D., et al., Dietary isoflavones differentially induce gene expression changes in lymphocytes from postmenopausal women who form equol as compared with those who do not. The Journal of Nutritional Biochemistry, 2007. 18(6): p. 380-390.

23. van der Velpen, V., et al., Isoflavone supplement composition and equol producer status affect gene expression in adipose tissue: a double-blind, randomized, placebo-controlled crossover trial in postmenopausal women. The American Journal of Clinical Nutrition, 2014.

24. Sartippour, M. R., et al., A pilot clinical study of short-term isoflavone supplements in breast cancer patients. Nutrition and Cancer, 2004. 49(1): p. 59-65.

25. Wu, A. H., et al., Double-blind randomized 12-month soy intervention had no effects on breast MRI fibroglandular tissue density or mammographic density. Cancer Prevention Research, 2015. 8(10): p. 942-951.

26. van der Velpen, V., et al., Estrogen receptor-mediated effects of isoflavone supplementation were not observed in whole-genome gene expression profiles of peripheral blood mononuclear cells in postmenopausal, equol-producing women. The Journal of Nutrition, 2013. 143(6): p. 774-780.

27. Cassidy, A., et al., Factors affecting the bioavailability of soy isoflavones in humans after ingestion of physiologically relevant levels from different soy foods. The Journal of Nutrition, 2006. 136(1): p. 45-51.

28. Oleson, L. and M. H. Court, Effect of the $\beta$-glucuronidase inhibitor saccharolactone on glucuronidation by human tissue microsomes and recombinant UDPglucuronosyltransferases (UGTS). The Journal of pharmacy and pharmacology, 2008. 60(9): p. 1175-1182.

29. Boonpawa, R., et al., Use of physiologically based kinetic (PBK) modeling to study interindividual human variation and species differences in plasma concentrations of quercetin and its metabolites. Biochemical Pharmacology, 2015. 98(4): p. 690-702.

30. Boonpawa, R., et al., A physiologically based kinetic (PBK) model describing plasma concentrations of quercetin and its metabolites in rats. Biochemical Pharmacology, 2014. 89(2): p. 287-299.

31. Steensma, A., H. P. J. M. Noteborn, and H. A. Kuiper, Comparison of Caco-2, IEC-18 and HCEC cell lines as a model for intestinal absorption of genistein, daidzein and their glycosides. Environmental Toxicology and Pharmacology, 2004. 16(3): p. 131-139.

32. Hosoda, K., et al., Plasma profiling of intact isoflavone metabolites by high-performance liquid chromatography and mass spectrometric identification of flavone glycosides daidzin and genistin in human plasma after administration of kinako. Drug Metabolism and Disposition, 2008. 36(8): p. 1485-1495. 
33. Setchell, K. D. R., et al., Bioavailability of pure isoflavones in healthy humans and analysis of commercial soy isoflavone supplements. The Journal of Nutrition, 2001. 131(4): p. 1362S1375 S.

34. Morito, K., et al., Interaction of phytoestrogens with estrogen receptors alpha and beta. Biological and Pharmaceutical Bulletin, 2001. 24(4): p. 351-356.

35. Hwang, C. S., et al., Isoflavone metabolites and their in vitro dual functions: They can act as an estrogenic agonist or antagonist depending on the estrogen concentration. The Journal of Steroid Biochemistry and Molecular Biology, 2006. 101(4-5): p. 246-253.

36. Yuan, J.-P., J.-H. Wang, and X. Liu, Metabolism of dietary soy isoflavones to equol by human intestinal microflora - implications for health. Molecular Nutrition \& Food Research, 2007. 51(7): p. 765-781.

37. Brown, R. P., et al., Physiological parameter values for physiologically based pharmacokinetic models. Toxicology and Industrial Health, 1997. 13(4): p. 407-484.

38. DeJongh, J., H. J. M. Verhaar, and J. L. M. Hermens, A quantitative property-property relationship (QPPR) approach to estimate in vitro tissue-blood partition coefficients of organic chemicals in rats and humans. Archives of Toxicology, 1997. 72(1): p. 17-25.

39. Simcyp. Simcyp prediction tools -fu. [Online]. Available from: https://members.simcyp.com/account/tools/fu/). [Accessed: 15th July 2015]. 2014 [cited 2014.

40. Simcyp. Simcyp prediction tools- blood to plasma partition ratio (B/P). [Online]. Available from: https://members.simcyp.com/account/tools/BP/). [Accessed: 15th July 2015]. 2014.

41. Evans, M. V. and M. E. Andersen, Sensitivity analysis of a physiological model for 2,3,7,8Tetrachlorodibenzo-p-dioxin (TCDD): Assessing the impact of specific model parameters on sequestration in liver and fat in the rat. Toxicological Sciences, 2000. 54(1): p. 71-80.

42. Louisse, J., et al., Prediction of in vivo developmental toxicity of all-trans-retinoic acid based on in vitro toxicity data and in silico physiologically based kinetic modeling. Archives of Toxicology, 2015. 89(7): p. 1135-1148.

43. Strikwold, M., et al., Combining in vitro embryotoxicity data with physiologically based kinetic (PBK) modelling to define in vivo dose-response curves for developmental toxicity of phenol in rat and human. Archives of Toxicology, 2013. 87(9): p. 1709-1723.

44. van der Woude, H., et al., The stimulation of cell proliferation by quercetin is mediated by the estrogen receptor. Molecular Nutrition \& Food Research, 2005. 49(8): p. 763-771.

45. Djiogue, S., et al., Estrogenic properties of naturally occurring prenylated isoflavones in U2OS human osteosarcoma cells: Structure-activity relationships. The Journal of Steroid Biochemistry and Molecular Biology, 2010. 120(4-5): p. 184-191.

46. Makela, S., et al., Dietary estrogens act through estrogen receptor-mediated processes and show no antiestrogenicity in cultured breast cancer cells. Environmental Health Perspectives, 1994. 102(6-7): p. 572-578.

47. Wang, T. T. Y., N. Sathyamoorthy, and J. M. Phang, Molecular effects of genistein on estrogen receptor mediated pathways. Carcinogenesis, 1996. 17(2): p. 271-275.

48. Murata, M., et al., Genistein and daidzein induce cell proliferation and their metabolites cause oxidative DNA damage in relation to isoflavone-induced cancer of estrogen-sensitive organs. Biochemistry, 2004. 43(9): p. 2569-2577.

49. Kuruto-Niwa, R., et al., Estrogenic activity of the chlorinated derivatives of estrogens and flavonoids using a GFP expression system. Environmental Toxicology and Pharmacology, 2007. 23(1): p. 121-128.

50. Rajah, T. T., et al., Genistein in the presence of $17 \beta$-estradiol inhibits proliferation of ER $\beta$ breast cancer cells. Pharmacology, 2009. 84(2): p. 68-73. 
Chapter 4

51. Gülden, M. and H. Seibert, In vitro-in vivo extrapolation: estimation of human serum concentrations of chemicals equivalent to cytotoxic concentrations in vitro. Toxicology, 2003. 189(3): p. 211-222.

52. Gülden, M., et al., Impact of protein binding on the availability and cytotoxic potency of organochlorine pesticides and chlorophenols in vitro. Toxicology, 2002. 175(1-3): p. 201213.

53. Shi, J. and H. Cao, Molecular structure-affinity relationship of dietary flavonoids for bovine serum albumin. Revista Brasileira de Farmacognosia, 2011. 21: p. 594-600.

54. EFSA, Guidance of the Scientific Committee on a request from EFSA on the use of the benchmark dose approach in risk assessment. The EFSA Journal, 2009. 1150: p. 1-72.

55. Argikar, U. A., Unusual Glucuronides. Drug Metabolism and Disposition, 2012. 40(7): p. 1239-1251.

56. Osborne, C. K., K. Hobbs, and J. M. Trent, Biological differences among MCF-7 human breast cancer cell lines from different laboratories. Breast Cancer Research and Treatment, 1987. 9: p. 111-121.

57. Evers, N. M., et al., Human T47D-ER $\beta$ breast cancer cells with tetracycline-dependent ER $\beta$ expression reflect ER $\alpha / E R \beta$ ratios in rat and human breast tissue. Toxicology in Vitro, 2013. 27(6): p. 1753-1761.

58. Bardin, A., et al., Loss of ERß expression as a common step in estrogen-dependent tumor progression. Endocrine-Related Cancer, 2004. 11(3): p. 537-551.

59. Boersma, M. G., et al., Regioselectivity of phase II metabolism of luteolin and quercetin by UDP-glucuronosyl transferases. Chemical Research in Toxicology, 2002. 15(5): p. 662-670.

60. Haakensen, V. D., et al., Expression levels of uridine 5'-diphospho-glucuronosyltransferase genes in breast tissue from healthy women are associated with mammographic density. Breast Cancer Research, 2010. 12(4): p. 1-11. 


\section{Supporting information}

Table S4.1. Physiological parameters in human ${ }^{1}$

\begin{tabular}{lll}
\hline Parameters & Symbol & Human \\
\hline Body weight (kg) & BW & 70 \\
Tissue volumes (\% body weight) & VLu & 1.4 \\
$\quad$ Lumen & VI & 0.9 \\
Small intestine & VL & 2.6 \\
Liver & VK & 0.4 \\
Kidney & VR & 4.0 \\
Rapidly perfused tissues & VS & 75.7 \\
Slowly perfused tissues & VB & 7.9 \\
Blood & QC & 310.4 \\
Cardiac output (l/h) & & \\
Blood flow to tissue (\% cardiac output) & QI & 18.1 \\
Small intestine & QL & 4.6 \\
Liver (exclude portal vein) & QK & 17.5 \\
Kidney & QR & 29.8 \\
Rapidly perfused tissues & QS & 30 \\
Slowly perfused tissues & Q & \\
\hline
\end{tabular}

${ }^{1}$ Physiological parameters were obtained from Brown et al. [37]. 
Chapter 4

\section{Human small intestine S9}

A.Glucuronidation of G-7G

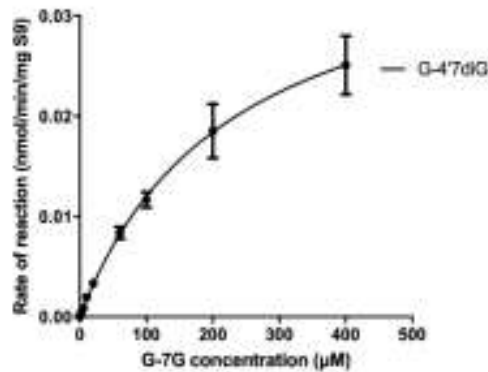

D. Sulfation of genistein

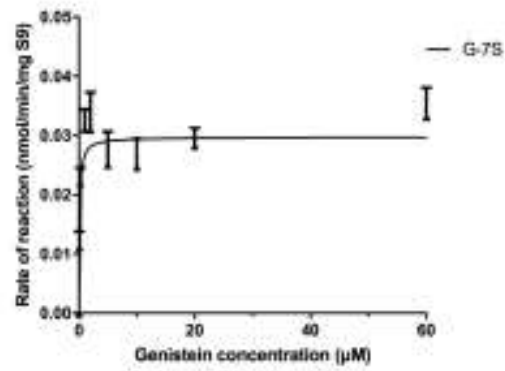

Human liver $\mathbf{9} 9$

F.Glucuronidation of $\mathrm{G}-4^{\prime} \mathrm{G}$

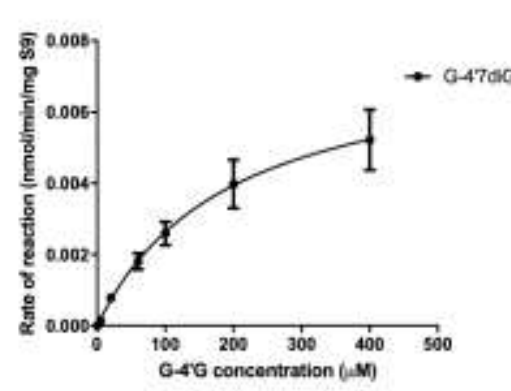

1.Sulfation of $\mathrm{G}-4^{\prime} \mathrm{G}$

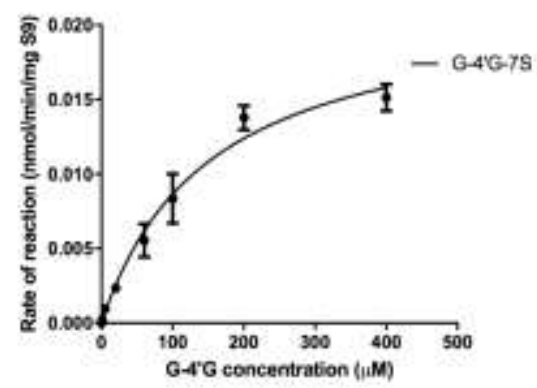

B.Glucuronidation of $\mathrm{G}-4$ 'G

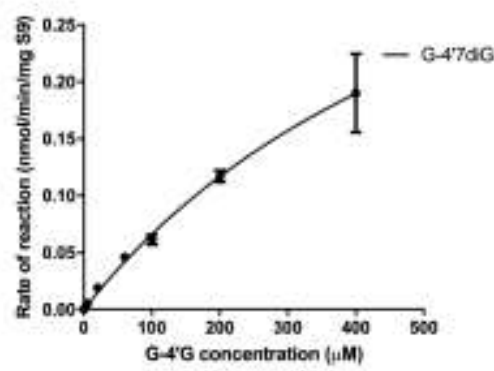

E.Sulfation of G-7G

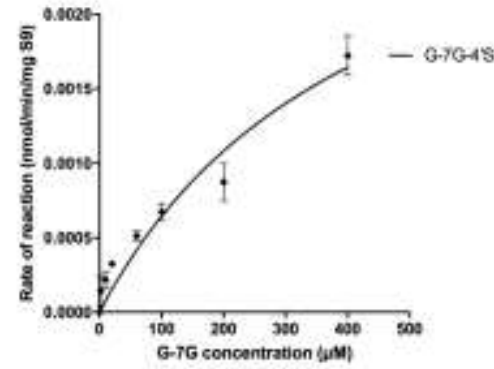

C.Glucuronidation of G-7S

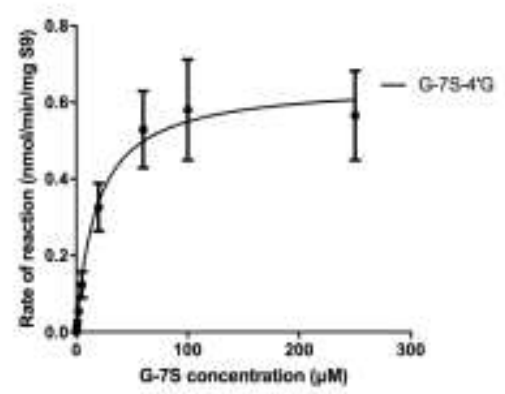

G. Sulfation of genistein

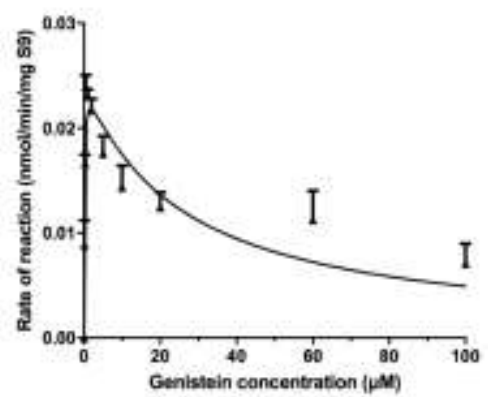

H.Sulfation of G-7G

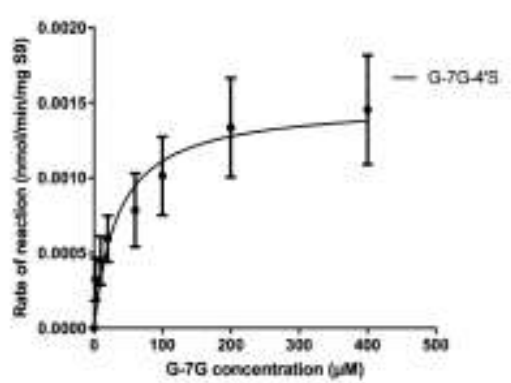

Figure S4.1 Concentration-dependent formation of genistein mono-conjugates and genistein diconjugates with pooled mixed-gender human small intestine S9 (A - E) or liver S9 (F - I): genistein7-O-glucuronide (G-7G), genistein-4'-O-glucuronide (G-4'G), genistein-7-O-sulfate (G-7S), genistein-4',7-O-diglucuronide (G-4'7diG), genistein-7-O-glucuronide-4'-O-sulfate (G-7G-4'S), and genistein-4'-O-glucuronide-7-O-sulfate (G-4'G-7S). 


\section{A. Genistein}

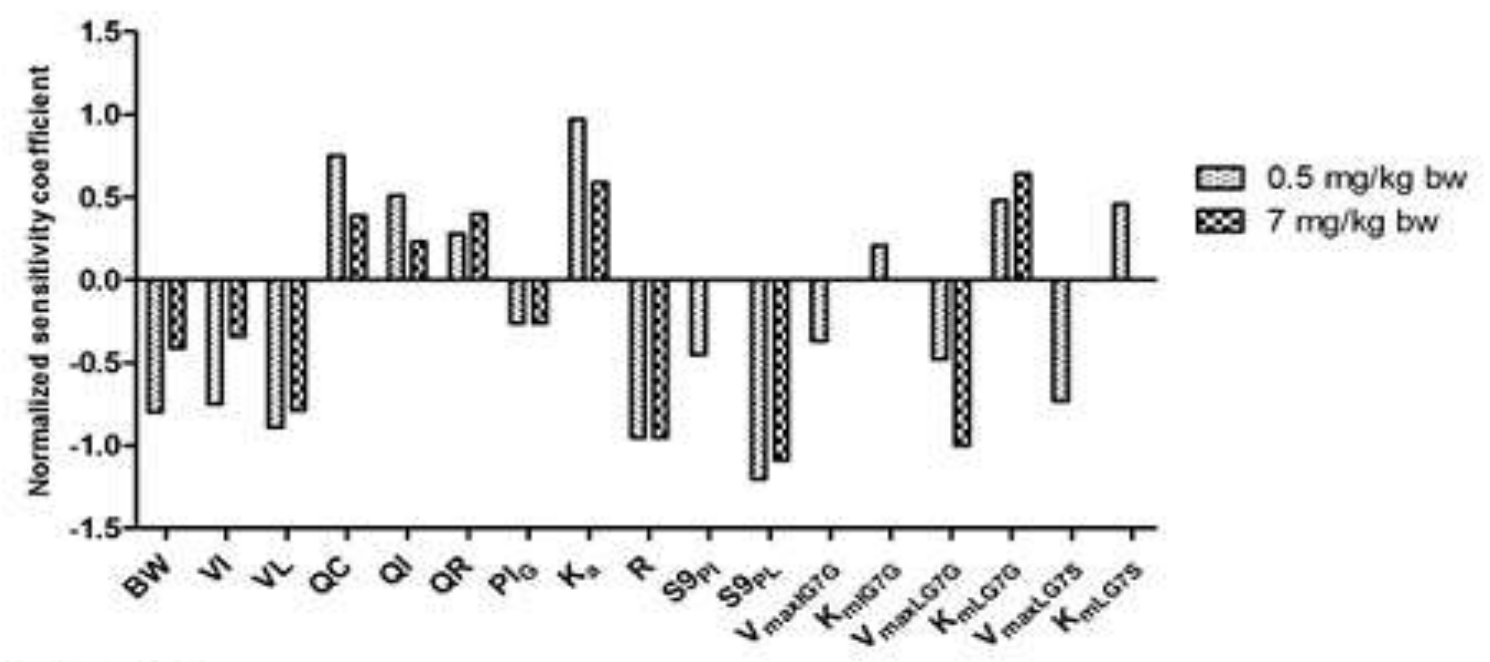

B. Genistin

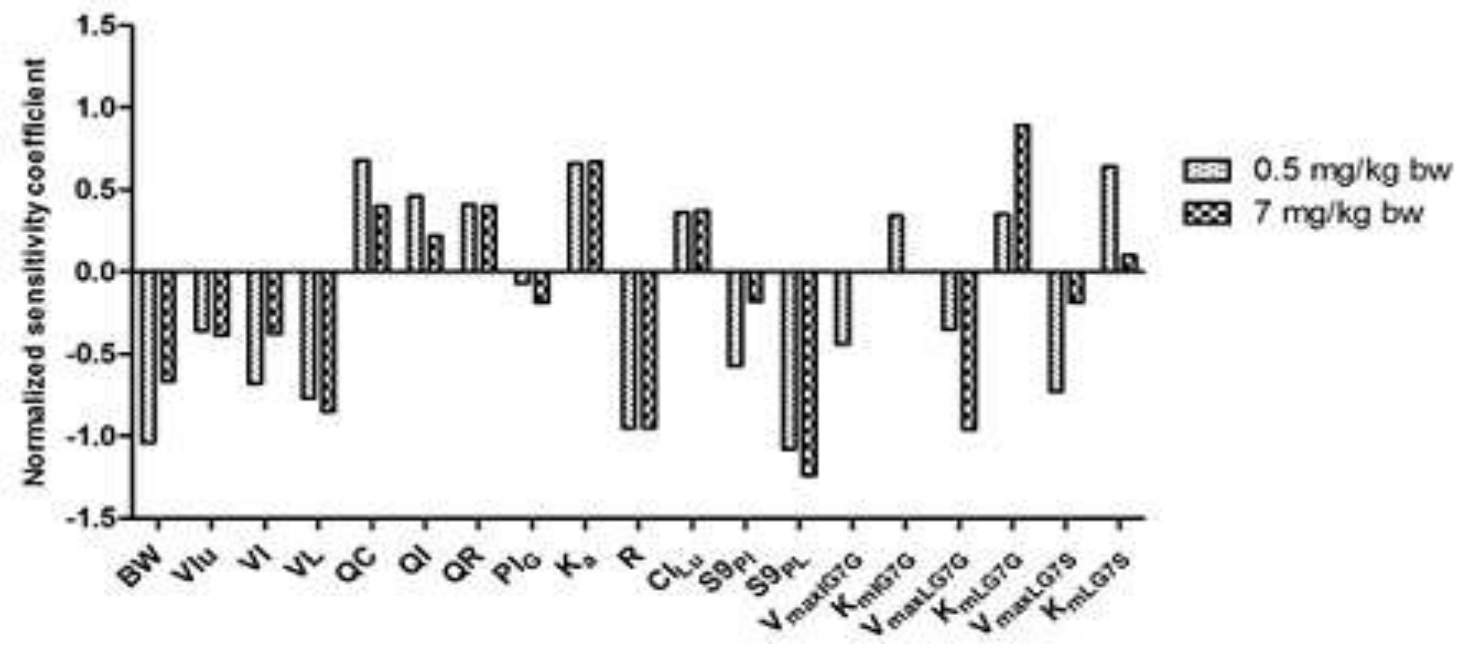

Figure S4.2 Sensitivity analysis of the predicted plasma $C_{\max }$ of genistein aglycone at an oral dose of 0.5 and $7 \mathrm{mg} / \mathrm{kg}$ bw (A) genistein and (B) genistin. The parameters stand for: BW = body weight, VTi = tissue volumn $(\mathrm{Ti}=\mathrm{I}$ (small intestine), $\mathrm{L}$ (liver), BR (breast), $\mathrm{R}$ (rapidly perfused tissues), and $\mathrm{S}$ (slowly perfused tissues), $\mathrm{QC}=$ cardiac output, $\mathrm{QTi}=$ reginal blood flow through tissue, $\mathrm{PTi}_{\mathrm{G}}=$ partition coefficient of genistein in tissue, $K_{\mathrm{a}}=$ uptake rate constant of genistein, $R=$ blood to plasma ratio, $\mathrm{S} 9_{\mathrm{PI}}=$ small intestinal $\mathrm{S} 9$ protein content, $\mathrm{S} 9_{\mathrm{PL}}=$ liver $\mathrm{S} 9$ protein content, $V_{\max }$ and $K_{\mathrm{m}}=$ the maximum rate of formation and the Michaelis-Menten constant for formation of genistein-7-Oglucuronide (G7G) and genistein-7-O-sulfate (G7S). 
Chapter 4

\section{A. ER $\alpha$ binding}
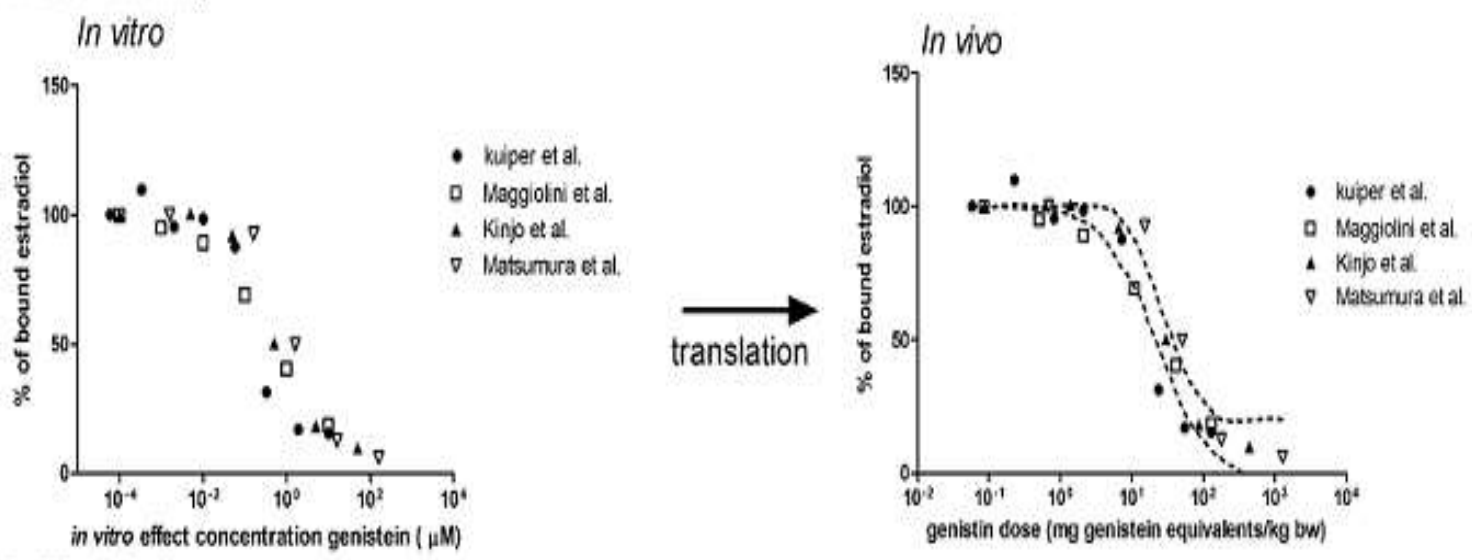

B. ER $\beta$ binding

In vitro

In vivo

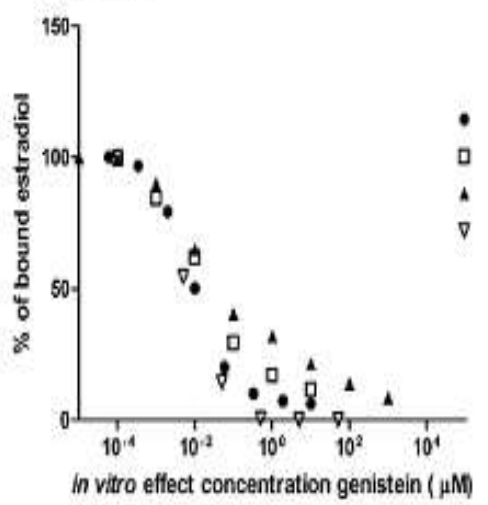

- kuiper et al

- Maggicini et al

- Han et al.

Kinjo et al.

C. ER $\alpha$ transcriptive induction
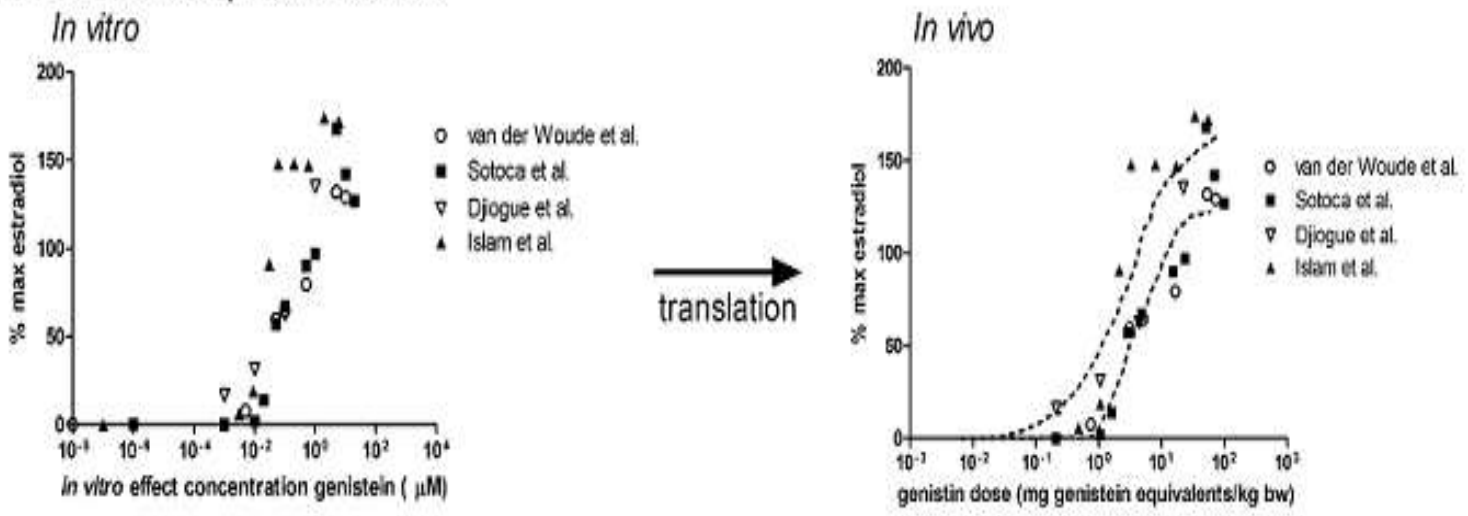

D. ER $\beta$ transcriptive induction
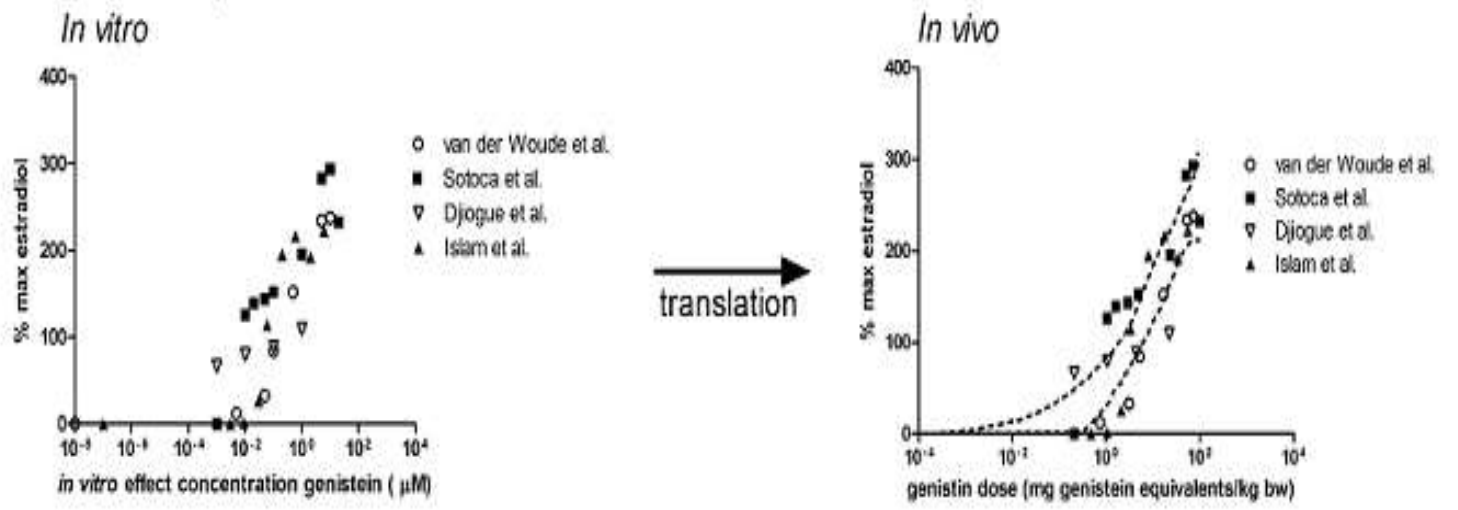


\section{E. ER $\alpha$ dependent proliferation}

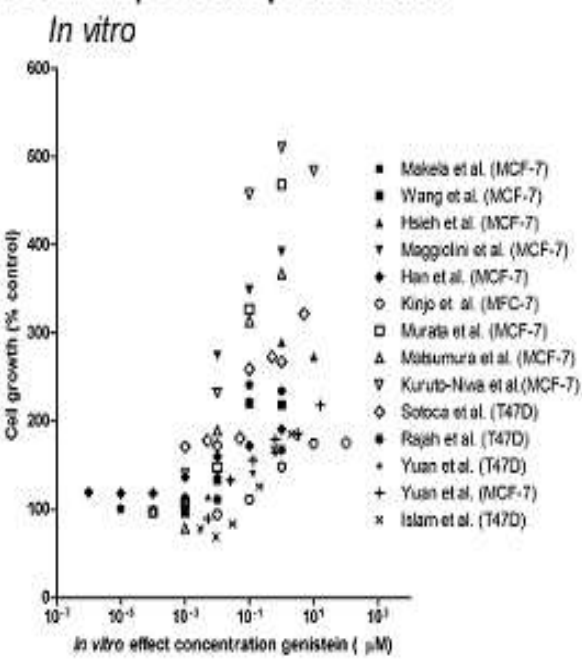

In vivo

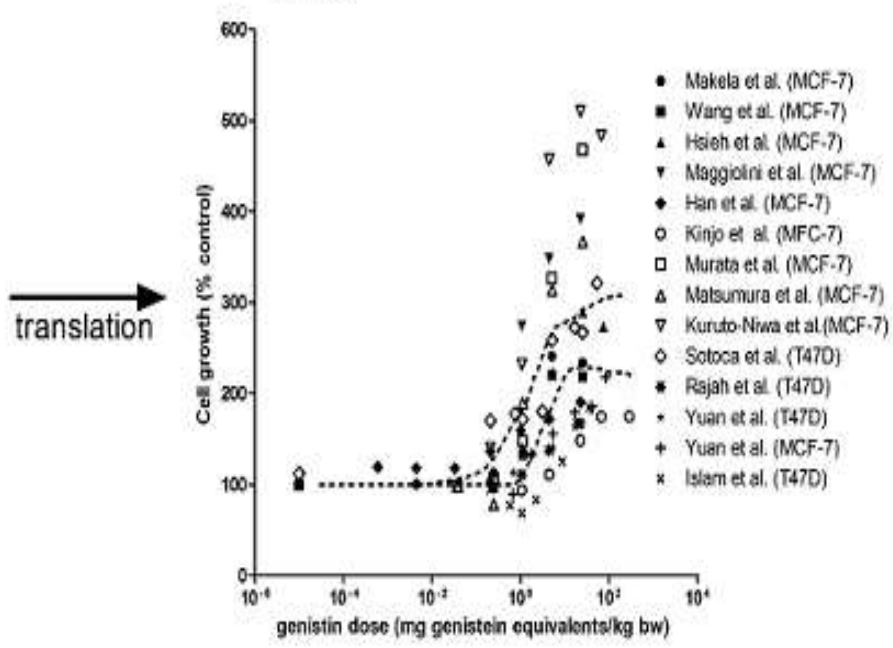

Figure S4.3 Translation of in vitro estrogenic concentration-response curves for genistein dependent (A) binding to ER $\alpha$ [4-7], (B) binding to ER $\beta$ [4-6, 8], (C) ER $\alpha$ mediated gene expression in U2OS reporter gene assays [10, 19, 44, 45], (D) ER $\beta$ mediated gene expression in U2OS reporter gene assays $[10,19,44,45]$, and (E) ER $\alpha$ dependent proliferation of MCF-7 and T47D breast cancer cells [5-10, 13, 19, 46-50], to in vivo human genistin dose-response curves using PBK model based reverse dosimetry based on plasma $C_{\max }$ values of genistein aglycone. The dashed lines represent the $95 \%$ lower and upper confidence limits. 
Chapter 4 


\section{CHAPTER 5}

Physiologically based kinetic modeling of hesperidin metabolism and its use to predict in vivo effective doses in humans

Rungnapa Boonpawa,

Albertus Spenkelink,

Ans Punt

Ivonne M. C. M. Rietjens,

Submission 


\begin{abstract}
The present study aimed to develop a physiologically based kinetic (PBK) model that describes the absorption, distribution, metabolism, and excretion (ADME) of hesperidin in humans, enabling the translation of in vitro concentration-response curves to in vivo doseresponse curves. Hesperidin was predicted to mainly occur in the systemic circulation as different monoglucuronides. The plasma concentration of hesperidin aglycone (hesperetin) was predicted to be $<0.02 \mathrm{mg} / \mathrm{l}$ at an oral dose of $50 \mathrm{mg} / \mathrm{kg}$ bw. The developed PBK model allowed conversion of in vitro concentration-response curves for different effects to predict in vivo dose-response curves from which $\mathrm{BMD}_{05}$ (benchmark dose for $5 \%$ response) values could be derived. The $\mathrm{BMD}_{05}$ values for PKA inhibition ranged between $135-529 \mathrm{mg} / \mathrm{kg}$ bw hesperidin, and for inhibition of endothelial cell migration and $\mathrm{PGE}_{2}$ and $\mathrm{NO}$ production ranged between $2.19-44 \mathrm{mg} / \mathrm{kg}$ bw hesperidin. These values are in line with reported human oral dose levels showing in vivo effects by hesperidin and show that these effects may occur at a Western dietary and a supplementary intake of hesperidin. It is concluded that the developed PBK model adequately predicts ADME of hesperidin in humans, and allows to evaluate the human in vivo situation without the need for human intervention studies.
\end{abstract}


A PBK based reverse dosimetry for in vivo effective hesperidin dose levels

\subsection{Introduction}

Hesperidin (hesperetin-7-O-rutinoside) is the $\beta$-glycoside form of the flavanone hesperetin, and is almost exclusively present in citrus fruits, especially in orange [1]. Sweet orange juice contains 487-584 mg hesperidin/l [2]. Hesperetin aglycone has been suggested to exert various beneficial health effects against cancer and cardiovascular diseases [3]. Available in vitro data reported that not only hesperetin aglycone, but also its metabolites could interact with various key proteins such as protein kinases [4-7] and induce or reduce specific genes related to cellular functions [8-10]. During uptake following oral ingestion, hesperidin as well as its aglycone hesperetin are extensively metabolized in intestinal and liver tissue and as a result hesperetin is mostly present in the systemic circulation in conjugated forms [11-14]. This raises the question of whether any in vivo biological effect of hesperidin or hesperetin is due to the free aglycone hesperetin or perhaps, at least in part, to these metabolites. Little is known about physiologically relevant concentrations of hesperetin aglycone and its circulating metabolites in vivo. For a better translation of in vitro results to in vivo effects, it is of importance to gain more insight in absorption, distribution, metabolism, and excretion (ADME) of hesperidin upon ingestion, especially to obtain an indication of the physiologically relevant plasma concentrations of hesperetin aglycone and its circulating metabolites.

Dietary intake of hesperidin in Western countries is estimated to be $193-562 \mathrm{mg}$ hesperidin corresponding to $2.8-8 \mathrm{mg} / \mathrm{kg}$ bw for a $70 \mathrm{~kg}$ person [15]. Hesperidin is also available as a food supplement with daily doses of 500-2000 $\mathrm{mg}$ hesperidin corresponding to $7-29 \mathrm{mg} / \mathrm{kg}$ bw for a $70 \mathrm{~kg}$ person [16]. Figure 5.1 illustrates possible metabolic pathways of dietary hesperidin in humans. Upon oral ingestion, hesperidin cannot be hydrolyzed by $\beta$ glucosidases in the small intestine, but passes to the colon, where it can be hydrolyzed to hesperetin aglycone by colonic microbiota prior to its absorption into the colonic enterocyte [12]. The release of hesperetin is the rate-limited step for absorption [14]. In contrast to hesperidin, hesperetin aglycone can be directly taken up in the small intestinal enterocyte by passive diffusion [12]. The absorbed hesperetin is metabolized by UDP-glucuronosyl transferases and sulfotransferases in the colon, small intestine and liver at the $3^{\prime}$ - and 7position [17]. Several metabolites of hesperetin including mono- and di-conjugates have been identified in human urine, indicating further conjugation of mono-conjugates $[11,13,18]$. 


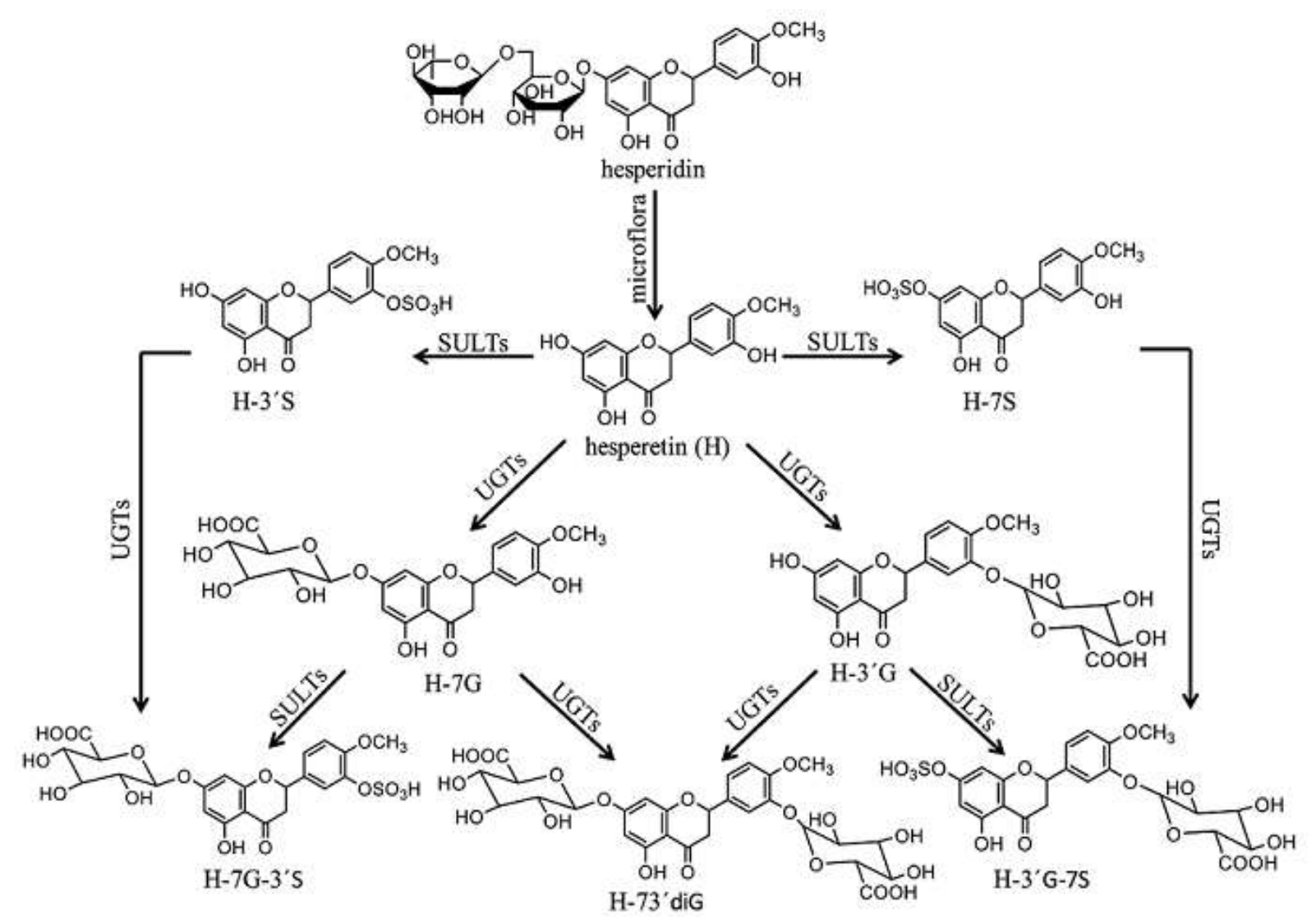

Figure 5.1 Proposed metabolic pathways of dietary hesperidin and hesperetin in humans: glucuronide (G) and sulfate $(\mathrm{S})[11,13,18]$.

To better understand in vivo effects of dietary hesperidin, the present study aimed to develop a physiologically based kinetic (PBK) model for hesperidin to gain insight in dosedependent plasma concentrations of hesperetin aglycone and its metabolites. The developed PBK model was subsequently combined with in vitro effect data using a so-called reverse dosimetry approach, to determine whether in vitro effects of hesperetin and its metabolites can also be expected to occur in vivo, and if so, at what hesperidin oral dose levels.

\subsection{Materials and methods}

\subsubsection{Materials}

Pooled mixed-gender human tissue fractions including small intestine microsomes, small intestine cytosol, liver microsomes, liver cytosol, and individual male colon S9 were purchased from Xenotech (Lenexa, USA). Uridine 5'-diphosphoglucuronide acid (UDPGA, purity 98\%), 3'-phosphoadenosine-5'-phosphosulfate (PAPS, purity 65\%), Tris, alamethicin (from Trichoderma viride), D-saccharic acid 1,4-lactone monohydrate, and DL-dithiothreitol (DTT) were purchased from Sigma-Aldrich (Steinheim, Germany). Hydrochloric acid (fuming 37\%), magnesium chloride, trifluoroacetic acid (for spectroscopy), potassium 
A PBK based reverse dosimetry for in vivo effective hesperidin dose levels

dihydrogen phosphate, dipotassium hydrogen phosphate trihydrate, and DMSO were purchased from VWR International (Darmstadt, Germany). Acetonitrile (chromatography grade) was purchased from Biosolve (Valkenswaard, The Netherlands). Hesperetin (>99\%) was purchased from Extrasynthese (Genay Cedex, France). Hesperetin-7-O-glucuronide (H7G) was purchased from Toronto Research Chemicals (Toronto, Canada). Hesperetin-3'-Oglucuronide $\left(\mathrm{H}-3^{\prime} \mathrm{G}\right)$ and hesperetin-3',7-O-diglucuronide (H-3’7diG) were purchased from Apollo Scientific (Cheshire, UK). Recombinant human cAMP-dependent protein kinase, catalytic subunit C $\alpha$ (PKA-C $\alpha$ ), kemptide (LRRASLG), ATP, and staurosporine were purchased from Biaffin (Kassel, Germany). ADP was purchased from DiscoveRx (Birmingham, UK).

5.2.2 Enzymatic conjugation of $\mathrm{H}-7 \mathrm{G}$ and $\mathrm{H}-3^{\prime} \mathrm{G}$ with pooled human tissue fractions To obtain kinetic parameters for glucuronidation and sulfation of the monoconjugates, $\mathrm{H}-7 \mathrm{G}$ and $\mathrm{H}-3^{\prime} \mathrm{G}$, to di-conjugates, required for the development of the PBK model, in vitro incubations with human tissue fractions were performed according to the methods described by Brand et al. [17] with some modifications. For glucuronidation, $100 \mu \mathrm{l}$ incubation mixtures were prepared containing (final concentrations) $10 \mathrm{mM} \mathrm{MgCl}, 10 \mathrm{mM}$ UDPGA, $0.025 \mathrm{mg} / \mathrm{ml}$ alamethicin (added from a 200 times concentrated stock solution in methanol), and human tissue fractions in $50 \mathrm{mM}$ Tris- $\mathrm{HCl}(\mathrm{pH}$ 7.4). For incubations with $\mathrm{H}-$ $7 \mathrm{G}$, a protein content of $0.6 \mathrm{mg} / \mathrm{ml}$ small intestine microsomes, $0.6 \mathrm{mg} / \mathrm{ml}$ liver microsomes, or $0.8 \mathrm{mg} / \mathrm{ml}$ colon $\mathrm{S} 9$ and incubation times of respectively 4,2 , or $6 \mathrm{~h}$ were applied. For incubations with $\mathrm{H}-3^{\prime} \mathrm{G}$, a protein content of $0.3 \mathrm{mg} / \mathrm{ml}$ small intestine microsomes, 1.0 $\mathrm{mg} / \mathrm{ml}$ liver microsomes, or $0.8 \mathrm{mg} / \mathrm{ml}$ colon $\mathrm{S} 9$ and incubation times of respectively 4,3 , or $6 \mathrm{~h}$ were applied. The reactions were initiated by adding H-7G or H-3'G from 200 times stock solutions in DMSO (final concentration of the substrate ranging from 5 to $400 \mu \mathrm{M}$ ) and were terminated by adding $25 \mu \mathrm{l}$ of ice-cold acetonitrile. Blank incubations were carried out without UDPGA. Under the specified conditions, glucuronidation was linear with time and protein content (data not shown).

For sulfation, $100 \mu \mathrm{l}$ incubation mixtures were prepared containing (final concentrations) $5 \mathrm{mM} \mathrm{MgCl} 2,0.1 \mathrm{mM}$ PAPS, $20 \mathrm{mM}$ saccharic acid-1,4-lactone (pH 7.4) as $\beta$-glucuronidase inhibitor [19], and human tissue fractions in $50 \mathrm{mM}$ potassium phosphate ( $\mathrm{pH}$ 7.4). For incubations with $\mathrm{H}-7 \mathrm{G}$, a protein content of $0.8 \mathrm{mg} / \mathrm{ml}$ small intestine cytosol, liver cytosol, or colon S9 and incubation times of respectively 3, 4, or $6 \mathrm{~h}$ were applied. For 


\section{Chapter 5}

incubations with $\mathrm{H}-3^{\prime} \mathrm{G}$, a protein content of $0.8 \mathrm{mg} / \mathrm{ml}$ small intestine cytosol, liver cytosol, or colon S9 and incubation times of 5, 4, or $6 \mathrm{~h}$ were applied. The reactions were initiated by adding $\mathrm{H}-7 \mathrm{G}$ or $\mathrm{H}-3^{\prime} \mathrm{G}$ from 200 times stock solutions in DMSO (final concentration of the substrate ranging from 5 to $400 \mu \mathrm{M}$ ) and were terminated by adding $25 \mu \mathrm{l}$ of ice-cold acetonitrile. Blank incubations were carried out without PAPS. Under the specified conditions, sulfation was linear with time and protein content (data not shown).

\subsubsection{Identification and quantification of hesperetin metabolites}

All incubation samples were analyzed on a UPLC-DAD system consisting of a Waters (Milford, MA) Acquity binary solvent manager, sample manager, and photodiode array detector, equipped with a Waters Acquity UPLC BEH C18 column $(1.7 \mu \mathrm{m}, 2.1 \times 50 \mathrm{~mm})$ at $40^{\circ} \mathrm{C}$ column temperature. All samples were centrifuged at $15000 \mathrm{rpm}$ for $5 \mathrm{~min}$ at $5^{\circ} \mathrm{C}$ to precipitate proteins and $3.5 \mu \mathrm{l}$ of the supernatant was subsequently analyzed using the UPLCDAD system. A gradient was applied consisting of nanopure water containing $0.1 \%$ trifluoroacetic acid (eluent A) and acetonitrile (eluent B) with a flow rate of $0.6 \mathrm{ml} / \mathrm{min}$ with the following profile: $0-25 \% \mathrm{~B}(0-1.0 \mathrm{~min}), 25 \% \mathrm{~B}(1.0-3.0 \mathrm{~min}), 25-50 \% \mathrm{~B}(3.0-4.0 \mathrm{~min})$, 50-80\% B (4.0-4.5 min), 80\% B (4.5-5.5 min), 80-0\% B (5.5-6.0 min) and 0\% B (6.0-7.0 $\min )$.

The diglucuronide, H-3'7diG, formed by glucuronidation of either $\mathrm{H}-7 \mathrm{G}$ orH-3'G was identified by comparing its UV spectrum and retention time with those of commercially available H-3'7diG. The nature of the sulfate metabolite of $\mathrm{H}-7 \mathrm{G}$ and $\mathrm{H}-3^{\prime} \mathrm{G}$ was confirmed by treating the unterminated incubation samples with $\beta$-glucuronidase/sulfatase as there was no commercially reference compound available. To this end, $50 \mu \mathrm{l}$ of unterminated incubation samples were added to $50 \mu \mathrm{l}$ of $0.1 \mathrm{M}$ sodium acetate ( $\mathrm{pH} 5.0)$ containing 189 units $/ \mathrm{ml} \beta$ glucuronidase and $1 \mathrm{unit} / \mathrm{ml}$ sulfatase. The reactions were carried out for $3 \mathrm{~h}$ at $37^{\circ} \mathrm{C}$ and were terminated by adding $25 \mu \mathrm{l}$ of ice-cold acetonitrile.

All formed metabolites were quantified by integrating the peak area at $280 \mathrm{~nm}$ and by using a calibration curve of $\mathrm{H}-3^{\prime} 7 \mathrm{diG}$. The sulfate metabolites of $\mathrm{H}-7 \mathrm{G}$ and $\mathrm{H}-3^{\prime} \mathrm{G}$ were quantified based on the calibration curve of $\mathrm{H}-3^{\prime} 7 \mathrm{diG}$, which was considered adequate because the sulfate metabolites of $\mathrm{H}-7 \mathrm{G}$ and $\mathrm{H}-3^{\prime} \mathrm{G}$ appeared to have the same UV-spectrum as $\mathrm{H}-3^{\prime} 7 \mathrm{diG}$ (data not shown) and were assumed to display a similar molar extinction coefficient. 
A PBK based reverse dosimetry for in vivo effective hesperidin dose levels

The kinetic parameters including the apparent maximum velocity $\left(V_{\max (\mathrm{app})}\right)$ and apparent Michaelis-Menten constant $\left(K_{\mathrm{m}(\mathrm{app})}\right)$ for different conjugation reactions were determined by fitting the data to the standard Michaelis-Menten equation with GraphPad Prism version 5.04 (GraphPad Software, San Diego, California, USA). The Michaelis-Menten equation was as follows:

$$
v=V_{\max } *[\mathrm{~S}] /\left(K_{\mathrm{m}}+[\mathrm{S}]\right)
$$

where [S] represents the substrate concentration. The values of $V_{\max }$ and $K_{\mathrm{m}}$ were expressed in $\mathrm{nmol} / \mathrm{min} / \mathrm{mg}$ protein and $\mu \mathrm{M}$, respectively.

\subsubsection{Protein kinase A (PKA) inhibition assay}

A PKA inhibition assay was carried to measure the potency of hesperetin and its monoglucuronides $\mathrm{H}-7 \mathrm{G}$ and $\mathrm{H}-3^{\prime} \mathrm{G}$ to inhibit PKA as possible mechanism underlying some of its beneficial effects [20,21]. The PKA inhibition assay was carried out based on the method described by Chen et al. [22] with some modifications. The incubation mixtures (final volume $50 \mu \mathrm{l}$ ) contained (final concentrations) $5 \mathrm{mM} \mathrm{MgCl}_{2}, 0.5 \mathrm{mM}$ DTT, $96 \mathrm{ng} / \mathrm{ml} \mathrm{PKA,} 4$ $\mu \mathrm{M}$ kemptide (PKA substrate), hesperetin, $\mathrm{H}-7 \mathrm{G}$, or $\mathrm{H}-3^{\prime} \mathrm{G}$ added from a 100 times concentrated stock in DMSO (final concentration ranging from 0 to $500 \mu \mathrm{M}$ ) in $50 \mathrm{mM}$ Tris$\mathrm{HCl}(\mathrm{pH}$ 7.4). The final concentration of DMSO was kept at $1 \%$ for all incubations. The positive control for PKA inhibition was carried out using $10 \mu \mathrm{M}$ staurosporine (final concentration), a potent PKA inhibitor with a reported $\mathrm{IC}_{50}$ value of $0.015 \mu \mathrm{M}$ [23]. The concentration of the substrate kemptide was selected to be close to the $K_{\mathrm{m}}$ value of $4.3 \mu \mathrm{M}$ at $5 \mu \mathrm{M}$ ATP for ADP induction (data not shown). The incubation mixtures were pre-incubated for $3 \mathrm{~min}$ at $30^{\circ} \mathrm{C}$ prior to initiation of the reactions by adding $5 \mu \mathrm{M}$ ATP (final concentration). After $30 \mathrm{~min}$, the reactions were terminated by adding $50 \mu \mathrm{l}$ of ice-cold acetonitrile. Under the specified conditions, ADP formation was linear with time and protein content (data not shown).

All samples were centrifuged at $15000 \mathrm{rpm}$ for $5 \mathrm{~min}$ at $5^{\circ} \mathrm{C}$ to precipitate proteins and $5 \mu 1$ of the supernatants were subsequently analyzed on a UPLC-DAD system consisting of a Waters (Milford, MA) Acquity binary solvent manager, sample manager, and photodiode array detector, equipped with a Waters Acquity UPLC BEH Amide column $(1.7 \mu \mathrm{m}, 2.1 \mathrm{x}$ $100 \mathrm{~mm}$ ) at $30^{\circ} \mathrm{C}$ column temperature. ADP, a byproduct from the phosphorylation reactions, was measured at isocratic conditions (70:30-acetonitrile: $27 \mathrm{mM} \mathrm{KH}_{2} \mathrm{PO}_{4}$ in nanopure water $\mathrm{pH} 4.5$ ) for $8 \mathrm{~min}$ and at a flow rate of $0.5 \mathrm{ml} / \mathrm{min}$. With this method, ADP eluted at $2.02 \mathrm{~min}$ 


\section{Chapter 5}

based on the retention time and UV spectrum of commercially available ADP. Triplicate experiments were performed. The inhibitory potency of hesperetin and its monoglucuronides was determined based on comparison to the kinase activity in a blank incubation performed without added inhibitor containing 1\% DMSO. The obtained concentration-response curves were translated to in vivo dose-response curve (Section 5.2.6)

\subsubsection{PBK model}

A PBK model for dietary hesperidin was developed in a similar manner as previously described for the development of a PBK model for quercetin $[24,25]$. The PBK model was defined to gain more insight in the ADME characteristics of hesperidin and the various metabolites formed in humans and to provide a method for conversion of in vitro effective concentrations to in vivo dose levels (Section 5.2.6). The model consisted of separate compartments for gastrointestinal (GI)-tract lumen, small intestine, colon, liver, kidney, rapidly perfused tissues (e.g. heart, lung, brain), and slowly perfused tissues (e.g. skin, muscle, bone) as shown in Figure 5.2A and 5.2B for the mono- and di-conjugates of hesperetin. Hesperidin was modeled to be hydrolyzed to its aglycone, hesperetin, by microbiota in the colon prior to absorption. The rate constants for hydrolytic clearance of hesperidin by the colonic microbiota, for excretion via urine and bile, and for the intestinal efflux back to the intestinal lumen were obtained by fitting with available experimental human data $[11,13,14,26-28]$, resulting in values of $0.1 \mathrm{l} / \mathrm{h}, 30 \mathrm{~h}^{-1}, 30 \mathrm{~h}^{-1}$, and $0.2 \mathrm{l} / \mathrm{h}$, respectively. The hesperetin that is released within the lumen was modelled to be absorbed into the colonic enterocyte by passive diffusion. The absorption rate constant for intestinal uptake of hesperetin is not available. An in situ intestinal perfusion study showed that the rate transfer of hesperetin to the intestinal wall was similar to that for genistein [29]. Based on this observation the absorption rate constant of hesperetin was assumed to be similar to that for genistein with a value of $5 \mathrm{~h}^{-1}$ [30]. To take the transit time of hesperidin from the stomach to the colon into account, the GI transit rates were included using the values reported by Kimura et al. [31] of 4.63, 2.44, $2.76 \mathrm{~h}^{-1}$ for stomach, jejunum, and ileum, respectively.

Hesperetin was modeled to primarily converse via glucuronidation and sulfation forming four different mono-conjugates at the $3^{\prime}$ - and 7-position in small intestine, colon, and liver. Further conjugation of different mono-conjugates to di-conjugates was simulated only for the mono-glucuronides, $\mathrm{H}-7 \mathrm{G}$ and $\mathrm{H}-3^{\prime} \mathrm{G}$, since these metabolites were estimated to be the major primary metabolites formed accounting for approximately $72 \%$ of the hesperidin dose 
A. mono-conjugates

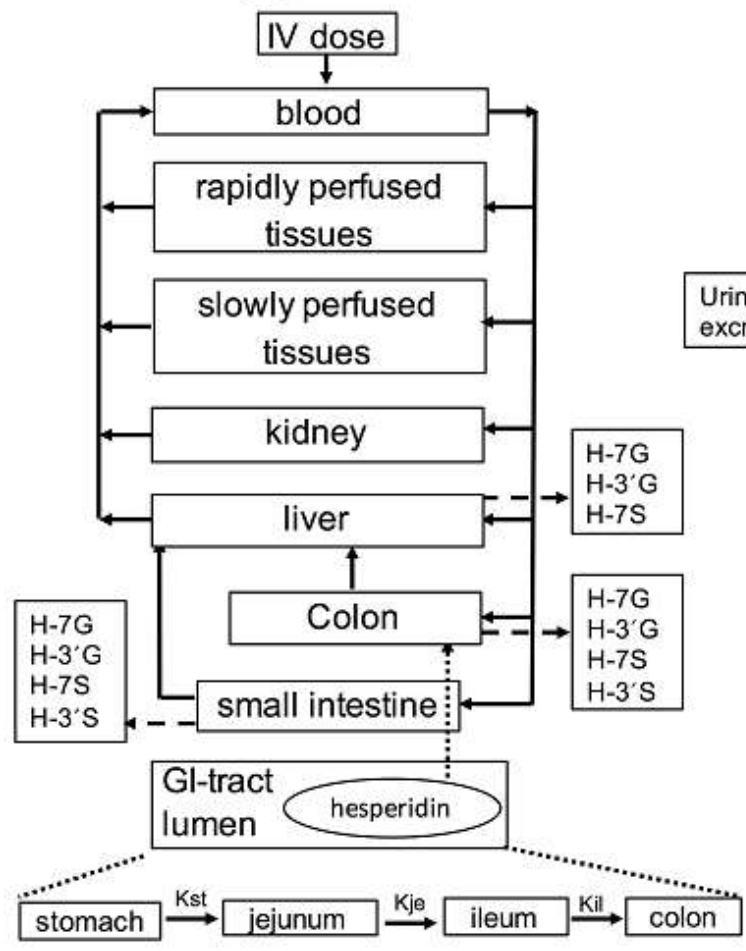

B. di-conjugates

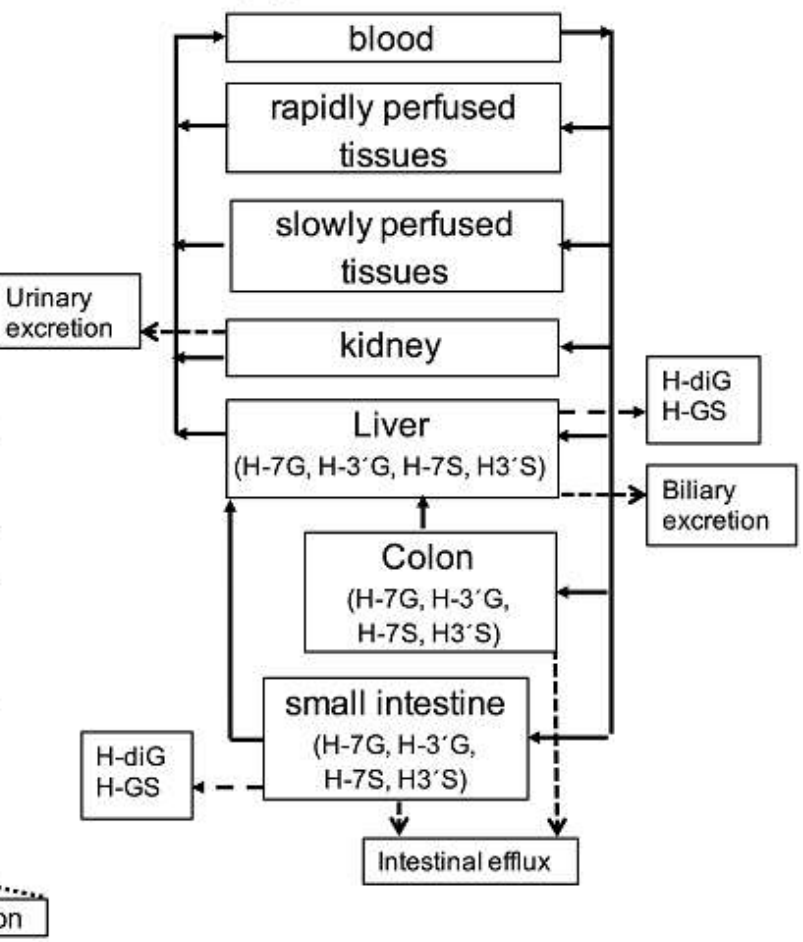

Figure 5.2 PBK model structure for formation of (A) mono- and (B) di-conjugates of hesperetin: $\mathrm{KTi}=$ transit rate constant for stomach (st), jejunum (je), and ileum (il), hesperetin-7-O-glucuronide

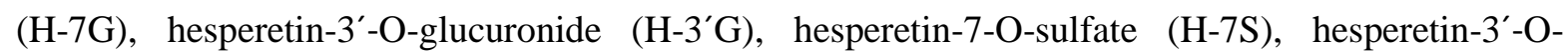
sulfate (H-3'S), hesperetin-3',7-O-diglucuronide (H-diG), and hesperetin-monoglucuronide-Omonosulfate (H-GS).

based on model stimulation (data not shown). The kinetic constants for formation of monoconjugates of hesperetin in small intestine, colon, and liver were taken from the study of Brand et al. [17] (Section 5.3.2). The kinetic constants for further conjugations of H-7G and $\mathrm{H}-3^{\prime} \mathrm{G}$ via glucuronidation and sulfation were obtained in the present study from in vitro incubations with relevant human tissue fractions (Section 5.3.1-5.3.2). The $V_{\max }$ values for formation of different mono- and di-conjugates were scaled to in vivo $V_{\max }$ values using a small intestine protein yield of $20.6 \mathrm{mg}$ microsomal protein and $18 \mathrm{mg}$ cytosolic protein [32] and a liver protein yield of $40 \mathrm{mg}$ microsomal protein and $80.7 \mathrm{mg}$ cytosolic protein [33]. The scaling factors for colonic metabolism were calculated based on the methods described by van de Kerkhof et al. [34] using the reported data that the colonic tissue of male F344 rat with 24 $\mathrm{mg}$ wet weight contained $3 \mathrm{mg}$ protein [35]. A microsomal protein yield of $2.4 \mathrm{mg}$ and a cytosol protein yield of $16.4 \mathrm{mg}$ were calculated and used as scaling factors for colonic metabolism.

The physiological parameters for humans were obtained from literature [36] and are shown in Table 5.1. The tissue:blood partition coefficients of hesperetin and its metabolites 


\section{Chapter 5}

Table 5.1 Physiological parameters in human ${ }^{1}$

\begin{tabular}{lll}
\hline Parameters & Symbol & Human \\
\hline Body weight (kg) & BW & 70 \\
Tissue volumes (\% body weight) & & \\
$\quad$ GI-tract lumen & VLu & 1.40 \\
Small intestine & VSI & 0.91 \\
Colon & VLI & 0.53 \\
Liver & VL & 2.60 \\
Kidney & VK & 0.4 \\
Rapidly perfused tissues & VR & 3.46 \\
Slowly perfused tissues & VS & 7.57 \\
Blood & VB & 7.9 \\
Cardiac output (1/h) & QC & 310.4 \\
Blood flow to tissue (\% cardiac output) & & \\
Small intestine & QSI & 9.05 \\
Colon & QLI & 9.05 \\
Liver (exclude portal vein) & QL & 4.6 \\
Kidney & QK & 17.5 \\
Rapidly perfused tissues & QR & 29.8 \\
Slowly perfused tissues & QS & 30 \\
\hline Physiological parameters were obtained from Brown et al $\left[\begin{array}{l}{[36]} \\
\hline\end{array}\right.$ & &
\end{tabular}

${ }^{1}$ Physiological parameters were obtained from Brown et al. [36].

were estimated from $\log K_{\text {ow }}$ according to the method described by DeJongh et al. [37] and are shown in Table 5.2. $\log K_{\mathrm{ow}}$ values were estimated from $\operatorname{Clog} P$ values obtained from Scifinder (American Chemical Society, USA) and ChemBioDraw Ultra 14.0 (CambridgeSoft, USA). Conversion of blood concentrations of hesperetin and its metabolites to plasma concentrations was done by dividing the blood concentrations by a blood/plasma ratio $(R)$. This value was calculated using the Simcyp prediction tool $[38,39]$ and amounted to 0.66 . Mass balance equations were numerically integrated in Berkeley Madonna version 8.3.18 (Macey and Oster, UC Berkeley, CA) using Rosenbrock's algorithm for stiff system. Mass balance equations for hesperetin and its metabolites were similar to those previously described by Boonpawa et al. [24, 25] for PBK models of quercetin. Model performance was evaluated by comparing the predicted concentrations of urinary profiles to the reported data in human urine $[1,11,40]$. Further evaluation was done by performing a sensitivity analysis to assess key parameters that contribute substantially to the maximum concentration $\left(\mathrm{C}_{\max }\right)$ of hesperetin aglycone and its metabolites in plasma at an oral dose of 2.8 and $29 \mathrm{mg} / \mathrm{kg} \mathrm{bw}$ hesperidin representing the lowest and the highest hesperidin intake from Western diet and food supplements $[15,16]$. Normalized sensitivity coefficients (SCs) were calculated for the model parameters according to the method described by Evens and Andersen as follows [41]: 
A PBK based reverse dosimetry for in vivo effective hesperidin dose levels

$$
\mathrm{SC}=\left(\mathrm{C}^{\prime}-\mathrm{C}\right) /\left(\mathrm{P}^{\prime}-\mathrm{P}\right) \times(\mathrm{P} / \mathrm{C})
$$

where $C$ is the initial value of the model output, $C^{\prime}$ is the modified value of the model output resulting from an increase in parameter value, $P$ is the initial parameter value, and $P^{\prime}$ is the modified parameter value, taking a $5 \%$ increase in its value.

Table 5.2 Physico-chemical parameters of hesperetin and its metabolites in human

\begin{tabular}{|c|c|c|c|c|c|c|c|c|c|c|}
\hline \multirow[b]{2}{*}{ Compound } & \multirow[b]{2}{*}{$C \log P^{1}$} & \multirow[b]{2}{*}{$p \mathbf{K a}^{2}$} & \multirow[b]{2}{*}{$\mathbf{f u}^{3}$} & \multirow[b]{2}{*}{$R^{4}$} & \multicolumn{6}{|c|}{ Tissue:blood partition coefficients ${ }^{5}$} \\
\hline & & & & & $\begin{array}{l}\text { Small } \\
\text { intestine }\end{array}$ & Colon & Liver & Kidney & $\begin{array}{l}\text { Rapidly } \\
\text { perfused } \\
\text { tissue }\end{array}$ & $\begin{array}{l}\text { Slowly } \\
\text { perfused } \\
\text { tissue }\end{array}$ \\
\hline Hesperetin & 1.94 & 7.49 & 0.12 & 0.72 & 2.32 & 2.32 & 2.32 & 1.38 & 1.38 & 1.73 \\
\hline $\mathrm{H}-7 \mathrm{G}$ & -0.18 & 2.74 & 0.31 & 0.61 & 0.63 & 0.63 & 0.63 & 0.80 & 0.80 & 0.77 \\
\hline $\mathrm{H}-3^{\prime} \mathrm{G}$ & 0.232 & 2.78 & 0.24 & 0.63 & 0.68 & 0.68 & 0.68 & 0.83 & 0.83 & 0.80 \\
\hline $\mathrm{H}-7 \mathrm{~S}$ & 0.67 & - & - & - & 0.79 & 0.79 & 0.79 & 0.88 & 0.88 & 0.86 \\
\hline H-3'S & 1.90 & -4.14 & 0.07 & 0.68 & 2.22 & 2.22 & 2.22 & 1.35 & 1.35 & 1.67 \\
\hline H-3'7diG & -1.83 & - & - & - & 0.59 & 0.59 & 0.59 & 0.76 & 0.76 & 0.75 \\
\hline H-7G-3'S & -1.01 & - & - & - & 0.60 & 0.60 & 0.60 & 0.75 & 0.75 & 0.75 \\
\hline H-3'G-7S & -1.25 & - & - & - & 0.60 & 0.60 & 0.60 & 0.75 & 0.75 & 0.75 \\
\hline $\begin{array}{l}{ }^{1} \text { CLog } P \text { value } \\
\text { ChemBioDrav } \\
{ }^{2} p \text { Ka values } \\
{ }^{3} \text { Fraction unb } \\
{ }^{4} \text { Blood/plasm } \\
{ }^{5} \text { Tissue:blood } \\
\text { DeJongh } \text { et al. }\end{array}$ & $\begin{array}{l}\text { for hesper } \\
\text { Jltra } 14.0 \\
\text { e obtaine } \\
\text { nd }(f u) w \\
\text { atio }(R) \mathrm{w} \\
\text { artition co } \\
\text { 37]. }\end{array}$ & $\begin{array}{l}\text { in and } \\
\text { Cambri } \\
\text { rom Sc } \\
\text { calcula } \\
\text { calcul } \\
\text { ficients }\end{array}$ & $\begin{array}{l}\text { netab } \\
\text { soft, } \\
\text { der ( } \\
\text { using } \\
\text { usin } \\
\text { hespe }\end{array}$ & $\begin{array}{l}\text { es wer } \\
\text { A). } \\
\text { erican } \\
\text { imcyp 1 } \\
\text { imcyp } \\
\text { in and }\end{array}$ & $\begin{array}{l}\text { stimated u } \\
\text { diction to } \\
\text { ediction to } \\
\text { metabolite }\end{array}$ & $\begin{array}{l}\text { ng Scifin } \\
\text { ety, USA } \\
\text {-fu [38]. } \\
\text { s-blood } \\
\text { were cal }\end{array}$ & $\begin{array}{l}\text { lasm } \\
\text { ated a }\end{array}$ & $\begin{array}{l}\text { tition ro } \\
\text { ding to }\end{array}$ & $\begin{array}{l}\text { 39]. } \\
\text { nethod d }\end{array}$ & ped by \\
\hline
\end{tabular}

\subsubsection{Translation of in vitro effect concentrations to in vivo dose levels}

PBK based reverse dosimetry was carried out to convert in vitro effect concentrations to predicted in vivo dose levels of hesperidin based on the methods described by Louisse et al. [42] and Strikwold et al. [43]. To this end, biological effects of hesperetin and its metabolites derived from in vitro bioassays were used including inhibition of PKA activity by hesperetin and its monoglucuronides (Section 5.3.5) and available in vitro literature data for biological effects of hesperetin and its mono-conjugates on inhibition of cell migration induced by TNF$\alpha$ in human aortic endothelial cells (HAEC) [44] and inhibition by hesperetin metabolite mixtures (mainly mono-conjugates) on prostaglandin $\mathrm{E}_{2}\left(\mathrm{PGE}_{2}\right)$ and nitric oxide (NO) production induced by LPS in RAW 264.7 cells [7]. These in vitro data also showed that not only hesperetin aglycone, but also its metabolites could exert similar biological effects with comparable potency, indicating that in vivo effects of hesperidin may be due to the mixtures of hesperetin aglycone and its metabolites rather than an individual compound. Therefore, the in vitro effect concentrations obtained for hesperetin and its metabolites for the respective 


\section{Chapter 5}

effects were added up to obtain the in vitro mixture effect concentrations by using the concept of concentration addition as follows [45]:

$$
\mathrm{EC} x_{\text {mix }}=\left(\sum_{i=1}^{n} \frac{p_{i}}{\operatorname{EC} x_{i}}\right)^{-1}
$$

where $\mathrm{EC} x_{\text {mix }}$ is the total concentration of the mixtures (in $\mathrm{mg}$ hesperetin equivalents/l) required to produce effect $x, n$ is the number of mixture components, $p_{i}$ is the relative fraction of chemical $i$ in the mixtures, and ECx $x_{i}$ is the concentration of $i$ that produces effect $x$ by itself. The $p_{i}$ values of hesperetin, $\mathrm{H}-3^{\prime} \mathrm{G}, \mathrm{H}-7 \mathrm{G}, \mathrm{H}-3^{\prime} \mathrm{S}$, and $\mathrm{H}-7 \mathrm{~S}$ in total plasma concentrations of hesperetin (aglycone and metabolites) were predicted to be $0.01,0.54,0.32$, 0.08 , and 0.05 based on model stimulation (data not shown).

Before making the in vitro to in vivo translation, it is of importance to realise that only the free fraction (fu) of the chemical will exert the effects which implies a correction is required for differences between protein and lipid binding in the in vitro bioassays and the in vivo situation. For the PKA inhibition activity that was performed using bioassay conditions without added protein and lipid, each nominal in vitro effect concentration $\left(\mathrm{EC}_{\text {in vitro }}\right)$ was extrapolated to an in vivo effective concentration $\left(\mathrm{EC}_{\text {in vivo }}\right)$ by dividing the $\mathrm{EC}_{\text {in vitro }}$ with the $\mathrm{fu}_{\text {in vitro }}$ assuming $100 \%$ availability in vitro. The average $\mathrm{fu}_{\text {in vitro }}$ of 0.27 for $\mathrm{H}-7 \mathrm{G}$ and $\mathrm{H}-3^{\prime} \mathrm{G}$ was used as approximately $70 \%$ of the total plasma hesperetin concentration (aglycone and metabolites) was predicted to be H-7G and H-3'G (see Table 5.2 in Section 5.2.5 and Section 5.3.4). For in vitro activities that were obtained from in vitro bioassays performed in the presence of culture medium with added protein and lipid, each $\mathrm{EC}_{\text {in vitro }}$ was extrapolated to an $\mathrm{EC}_{\text {in vivo }}$ according to the extrapolation rule of Gülden and Seibert as follows [46]:

$$
\mathrm{EC}_{\text {in vivo }}=\mathrm{EC}_{\text {in vitro }} \mathrm{x}\left[\left(1-\mathrm{fb}_{\text {in vitro }}\right) \times \frac{1+\mathrm{K}_{\mathrm{OW}} \times \mathrm{V}_{\mathrm{L}, \text { serum }}^{\prime}}{1+\mathrm{K}_{\mathrm{OW}} \mathrm{VV}_{\mathrm{L}, \text { in vitro }}}+\mathrm{fb}_{\text {in vitro }} \times \frac{\mathrm{P}_{\text {serum }}}{\mathrm{P}_{\text {in vitro }}}\right]
$$

where $\mathrm{fb}_{\text {in vitro }}$ is the fraction of chemical bound to protein and lipid in culture medium, which is calculated from $1-\mathrm{fu}_{\text {in vitro }} . \mathrm{V}_{\mathrm{L}}^{\prime}$ is the lipid fraction in serum and in vitro. $\mathrm{P}$ is the protein content in serum and culture medium.

For the extrapolation, in vivo albumin and lipid concentrations of $600 \mu \mathrm{M}$ and $6 \mathrm{~g} / \mathrm{l}$ were used [46]. The albumin and lipid concentrations in culture medium of the reported in vitro bioassays were estimated from the reported data by Gülden et al. [47] indicating that 5\% foetal bovine serum contains $18 \mu \mathrm{M}$ bovine serum albumin and $0.1 \mathrm{~g} / \mathrm{l}$ lipid. The $\mathrm{fu}_{\text {in vitro }}$ values of hesperetin and its metabolites in in vitro medium were estimated using the reported binding constant of hesperetin, $\mathrm{H}-7 \mathrm{G}$, and $\mathrm{H}-3^{\prime} \mathrm{G}$ to human serum albumin of 8,4 , and $6 \times 10^{4}$ $\mathrm{M}^{-1}$ [48] and a binding site number of 1.05 [49], assuming a similar binding constant for 
A PBK based reverse dosimetry for in vivo effective hesperidin dose levels

human and bovine serum albumin. This assumption is based on the fact that genistein has been reported to have a similar binding constant for bovine and human serum albumin [50]. The in vivo effect concentration of the mixtures obtained was set equal to plasma $\mathrm{C}_{\max }$ of the mixtures in the PBK model to predict the corresponding oral dose level in humans. Subsequently, a dose-response curve was derived and used to estimate the benchmark dose for $5 \%$ response $\left(\mathrm{BMD}_{05}\right)$ based on the default benchmark response for continuous data [51]. The $\mathrm{BMD}_{05}$ values were estimated by fitting to the Hill model using Benchmark Dose Software version 2.6 (The Environment Protection Agency's, USA). The BMD 05 values obtained were evaluated against reported active human data on anti-atherogenic and antiinflammatory activity upon oral intake of hesperidin [52-54].

\subsection{Results}

5.3.1 Enzymatic conjugation of $\mathrm{H}-7 \mathrm{G}$ and $\mathrm{H}-3^{\prime} \mathrm{G}$ in incubations with human tissue fractions

Analysis of glucuronidation incubations of $\mathrm{H}-7 \mathrm{G}$ and $\mathrm{H}-3^{\prime} \mathrm{G}$ containing pooled human tissue fractions indicated that $\mathrm{H}-7 \mathrm{G}$ was further glucuronidated by human small intestine and liver microsomes, revealing one metabolite that is formed. Whereas $\mathrm{H}-3^{\prime} \mathrm{G}$ was only further glucuronidated by human liver microsomes, resulting in the same metabolite as observed from the incubations with H-7G. This metabolite co-eluted with and showed similar spectral characteristics as commercially available $\mathrm{H}-3^{\prime} 7 \mathrm{diG}$ and could thus be identified as $\mathrm{H}-3^{\prime} 7 \mathrm{diG}$. Under the conditions of the present study no glucuronidated metabolites of $\mathrm{H}-7 \mathrm{G}$ or $\mathrm{H}-3^{\prime} \mathrm{G}$ were formed in incubations containing human colon S9.

Analysis of sulfation incubations of $\mathrm{H}-7 \mathrm{G}$ and $\mathrm{H}-3^{\prime} \mathrm{G}$ containing pooled human tissue fractions revealed that $\mathrm{H}-7 \mathrm{G}$ was further sulfated by human small intestine and liver cytosol, while H-3'G was only further sulfated by human small intestine cytosol. The metabolites formed in the sulfation incubations with $\mathrm{H}-7 \mathrm{G}$ and $\mathrm{H}-3^{\prime} \mathrm{G}$ with a retention time of 1.3 and 1.4 min were identified by treating the unterminated samples with $\beta$-glucuronidase/sulfatase resulting in a disappearance of the respect peak and a concomitant equivalent increase in the peak area of hesperetin aglycone (data not shown). These results confirm that the metabolites were most likely H-7G-3'S and H-3'G-7S because conjugation of the flavonoid likely occurs at available free $\mathrm{OH}$ moieties [55] and the 5-position is not available for conjugation due to intramolecular hydrogen bonding with the carbonyl group at the C-4 position [56]. Under the conditions of the present study no sulfated metabolites of $\mathrm{H}-7 \mathrm{G}$ and $\mathrm{H}-3^{\prime} \mathrm{G}$ formed in incubations with human colon S9 were detected. 


\section{Chapter 5}

\subsubsection{Kinetic constants for formation of mono- and di-conjugates of hesperetin}

The concentration-dependent rates of glucuronidation and sulfation of $\mathrm{H}-7 \mathrm{G}$ and $\mathrm{H}-$ 3'G by human tissue fractions are shown in Figure S5.1 in the Supporting information. The kinetic constants derived from these results and the kinetic constants for formation of different hesperetin mono-conjugates obtained from Brand et al. [17] are summarized in Table 5.3. The results indicate that small intestine and liver had similar catalytic efficiency for metabolism of hesperetin with the total catalytic efficiency amounting to approximately 2.5 $\mathrm{l} / \mathrm{h} / \mathrm{g}$ tissue for both tissues, followed by colon with a total catalytic efficiency of $0.17 \mathrm{l} / \mathrm{h} / \mathrm{g}$ tissue. Small intestine and liver tissues showed a difference in the type of conjugation of hesperetin as small intestine appeared to be especially effective in formation of $\mathrm{H}-3^{\prime} \mathrm{S}$, whereas liver was more effective in converting hesperetin into $\mathrm{H}-3^{\prime} \mathrm{G}$. The formation of diconjugates was more than 100-fold less efficient than the formation of the respective monoconjugates due to a decrease in $V_{\max }$ and an increase in $K_{\mathrm{m}}$ values. Colon could only covert hesperetin to mono-conjugates as there was no formation of di-conjugates detected in incubations with this tissue fraction.

\subsubsection{PBK model development and evaluation}

To define the PBK model for hesperidin, kinetic parameters for formation of different hesperetin mono- and di-conjugates were obtained from in vitro incubations. While the hydrolytic clearance of hesperidin by gut microbiota and the kinetic parameters for excretion via urine, bile, and intestinal efflux back to intestinal lumen were obtained by fitting the predicted dose-dependent plasma concentrations of total hesperetin concentrations with available experimental human data in plasma [11, 13, 14, 26-28], resulting in values of 0.1 $1 / \mathrm{h}, 30 \mathrm{~h}^{-1}, 30 \mathrm{~h}^{-1}$, and $0.2 \mathrm{l} / \mathrm{h}$, respectively. Subsequently the model thus defined was used to predict the total hesperetin plasma kinetics as shown in Figure 5.3. The model predicted plasma $\mathrm{C}_{\max }$, AUC and $\mathrm{T}_{\max }$ of total hesperetin within 1- to 6-fold deviation from the reported human data.

The performance of the defined PBK model was evaluated to a further extent by comparing the predicted concentrations of hesperetin metabolites excreted in urine to the reported literature data for hesperetin metabolites in human urine [1, 11, 40]. Figure 5.4 shows the results of this comparison revealing that the model adequately predicted the urinary profiles of different mono- and di-conjugates of hesperetin within 1- to 7-fold deviation from the reported urinary profiles in humans $[1,11,40]$. 
A.

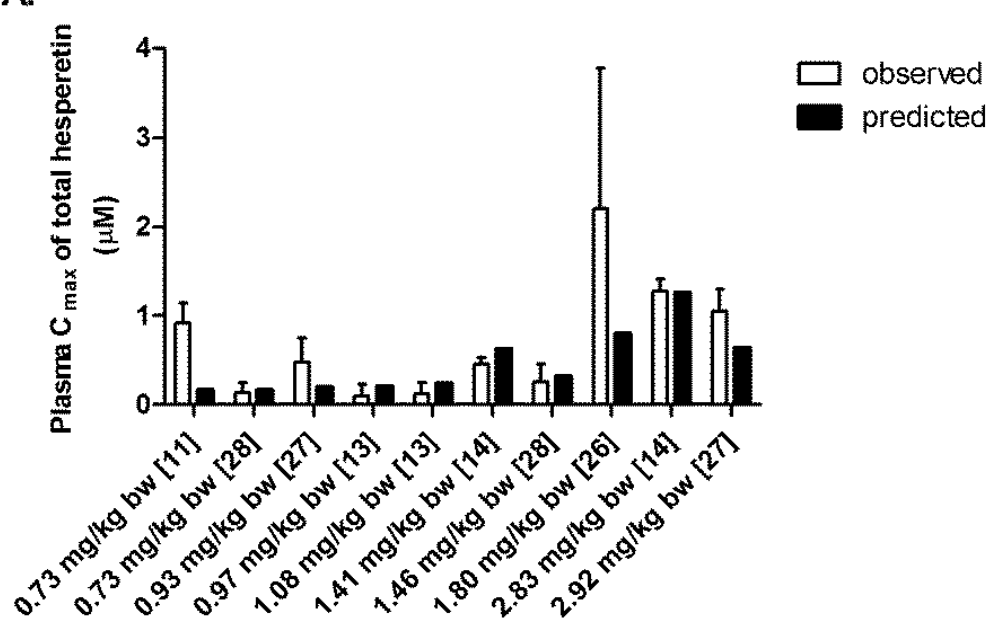

B.

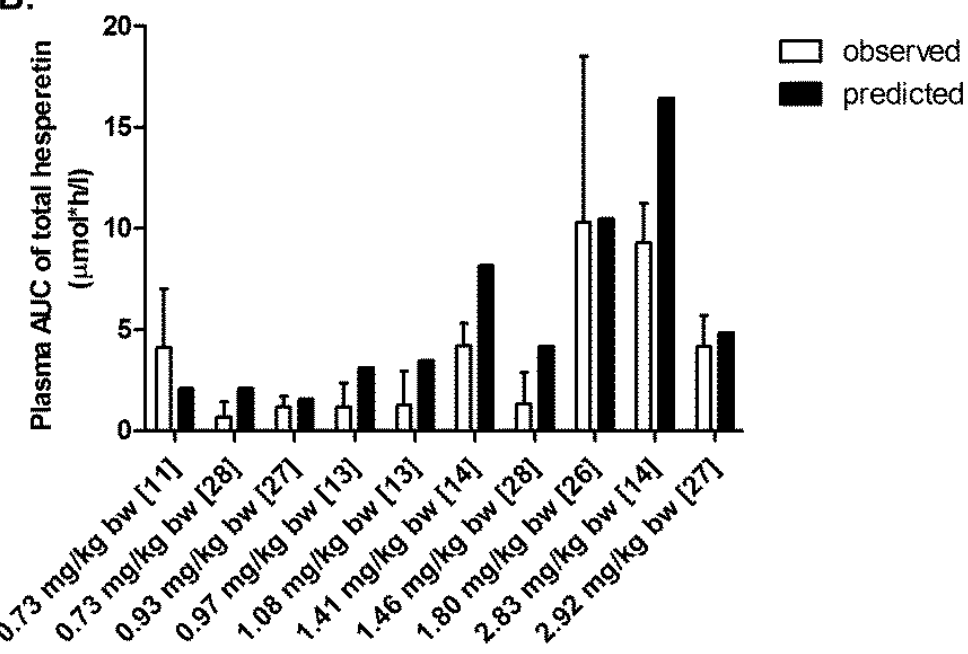

c.

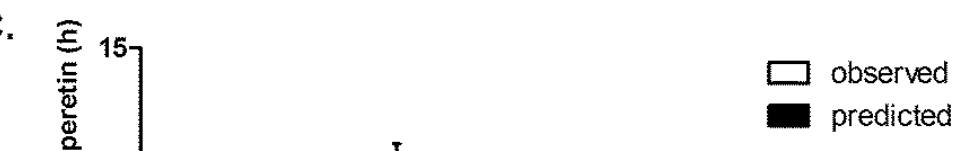

Figure 5.3 Observed and PBK model-predicted (A) plasma $C_{\max }$, (B) plasma AUC, and (C) plasma $\mathrm{T}_{\max }$ of total hesperetin (aglycone and metabolites) in humans at different dose levels as indicated by the $\mathrm{x}$-axis labels $[11,13,14,26-28]$. 


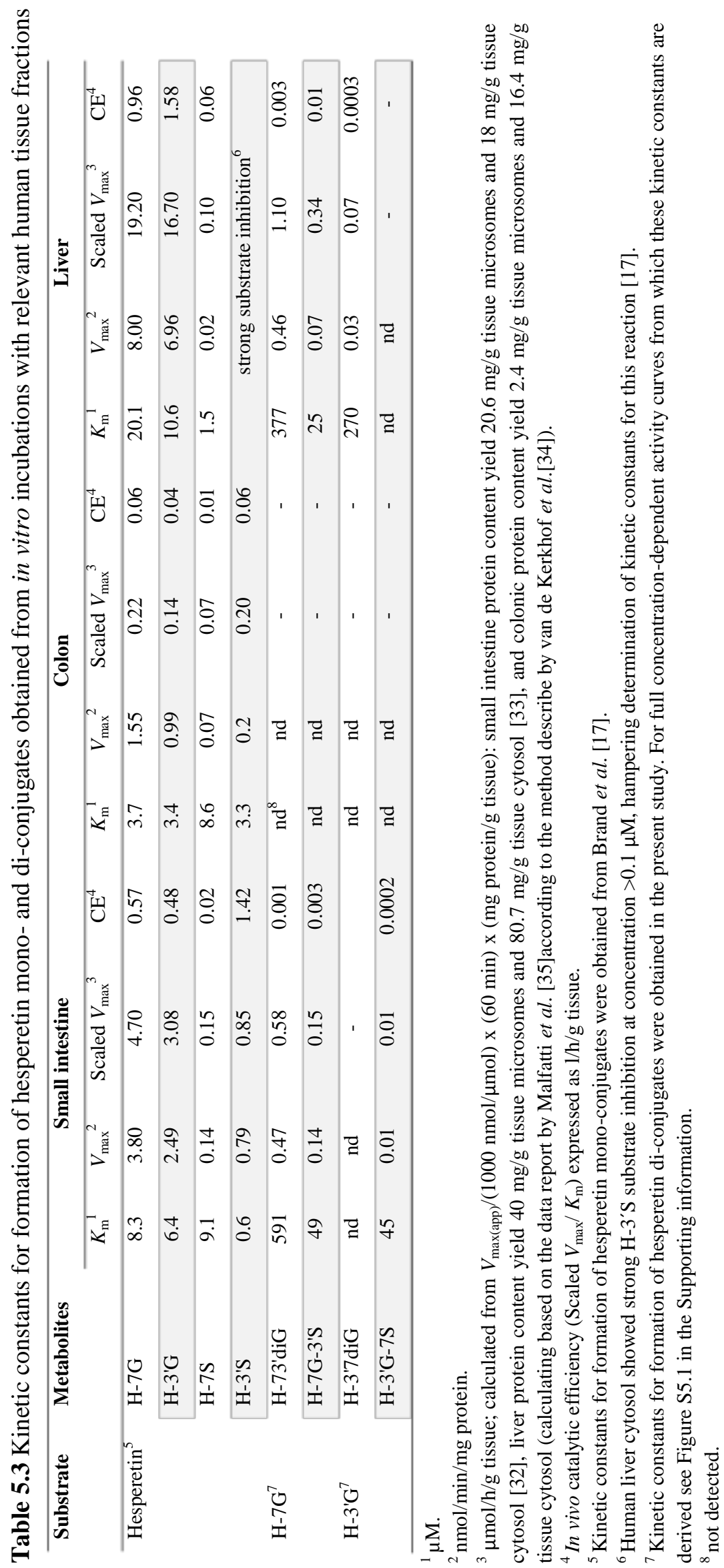



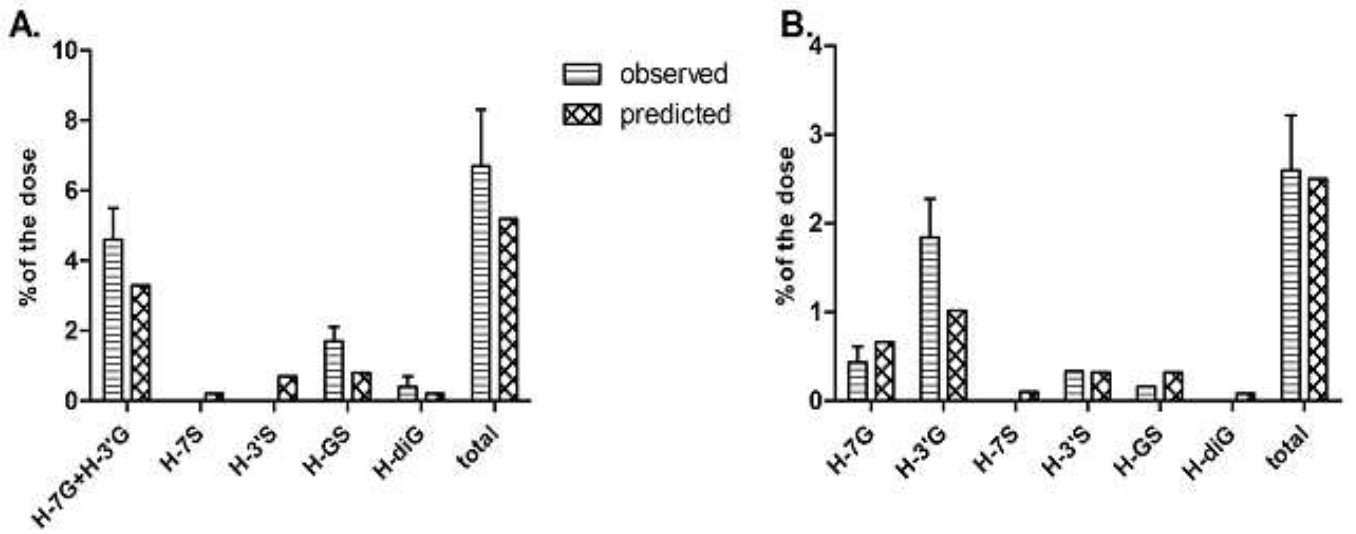

observed

区 predicted

C.

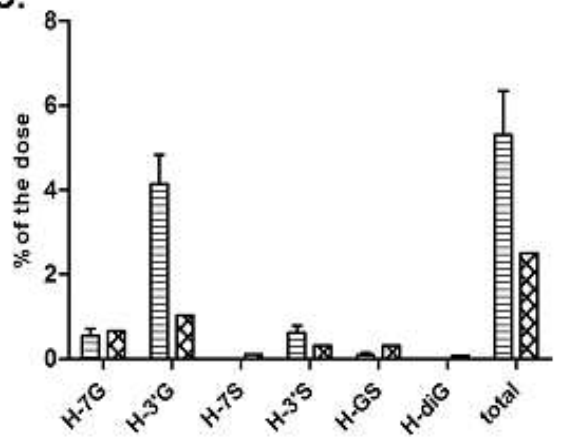

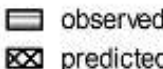

D. 2

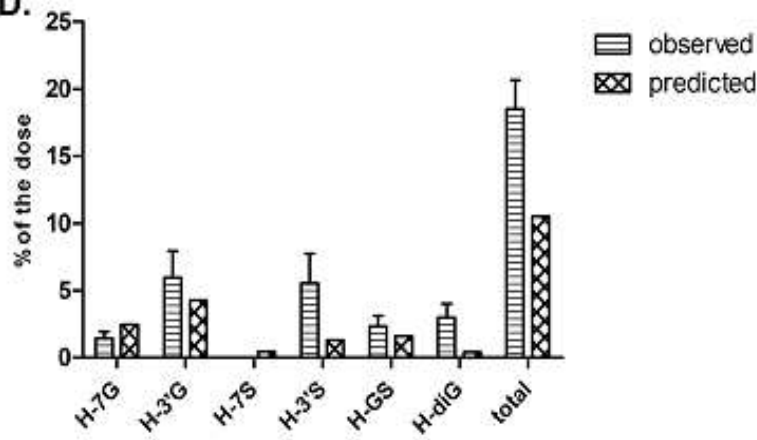

Figure 5.4 Model evaluation by comparing the PBK model-predicted urinary profiles with those reported by (A) Mullen et al. at $0.73 \mathrm{mg} / \mathrm{kg}$ bw hesperidin [11], (B) Bredsdorff et al. at $0.93 \mathrm{mg} / \mathrm{kg}$ bw hesperidin [40], (C) Bredsdorff et al. at $2.92 \mathrm{mg} / \mathrm{kg}$ bw hesperidin [40], and (D) Pereira-Caro et al. at $2.87 \mathrm{mg} / \mathrm{kg}$ bw hesperidin [1].

To further evaluate the performance of the defined model, a sensitivity analysis was performed to assess key parameters that influence the predicted plasma $\mathrm{C}_{\max }$ of hesperetin aglycone and its metabolites at an oral dose of 2.8 and $29 \mathrm{mg} / \mathrm{kg}$ bw hesperidin representing the lowest and the highest hesperidin intake originating from the Western diet and from food supplements, respectively $[15,16]$. Only parameters that had a normalized sensitivity coefficient higher than 0.1 (in absolute value), are shown in Figure S5.2 in the Supporting information. The results reveal that the normalized sensitivity coefficients of most of the PBK model parameters were not dose-dependent. The predicted plasma $\mathrm{C}_{\max }$ of hesperetin aglycone and its metabolites are mainly influenced by the body weight, the volume of the GI-tract lumen and liver, the reginal blood flow through the small intestine and colon, the blood to plasma ratio, and the biliary excretion (expressing normalized sensitivity coefficients between 0.5 to 2.6 in absolute value). The parameters that influenced the formation of hesperetin mono-conjugates in the colon and liver, including the microsomal and cytosol protein contents in the colon and liver and the kinetic constants for formation of mono-conjugates, are also influential parameters for the predicted plasma $\mathrm{C}_{\max }$ of hesperetin aglycone and its 


\section{Chapter 5}

metabolites (expressing normalized sensitivity coefficients between 0.2 to 0.9 in absolute value). In addition, the kinetic constants for formation of $\mathrm{H}-3^{\prime} 7 \mathrm{diG}$ and $\mathrm{H}-7 \mathrm{G}-3^{\prime} \mathrm{S}$ in the liver and the kinetic constants for formation of $\mathrm{H}-3^{\prime} \mathrm{G}-7 \mathrm{~S}$ and the cytosol protein content in the small intestine are also influential parameters for the predicted plasma $\mathrm{C}_{\max }$ of its respective di-conjugates (expressing normalized sensitivity coefficients between 0.3 to 2.0 in absolute value).

\subsubsection{PBK model predictions of metabolic plasma profiles of hesperidin}

The PBK model was used to predict metabolic plasma profiles of hesperidin at different dose levels. Figure 5.5 shows the predicted dose-dependent plasma concentrations of hesperetin aglycone and its metabolites (expressed in $\mathrm{mg}$ hesperetin equivalents/l) at oral doses ranging from $0.01-50 \mathrm{mg} / \mathrm{kg}$ bw hesperidin, revealing that the conjugation pattern of the circulating metabolites does not change with the dose. At all oral doses, H-3'G and H-7G were predicted to be the major circulating metabolites together accounting for approximately $70 \%$ of the total plasma hesperetin concentration (aglycone and metabolites), followed by $\mathrm{H}$ 7G-3'S, H-3'S, and H-3'7diG accounting for approximately 15, 6, and $4 \%$ of the total plasma hesperetin concentration, respectively. Hesperetin aglycone was present at a low concentration below $0.02 \mathrm{mg} / \mathrm{l}$ at a dose of $50 \mathrm{mg} / \mathrm{kg}$ bw and accounted for approximately $0.5 \%$ of the total plasma hesperetin concentration at all oral doses.

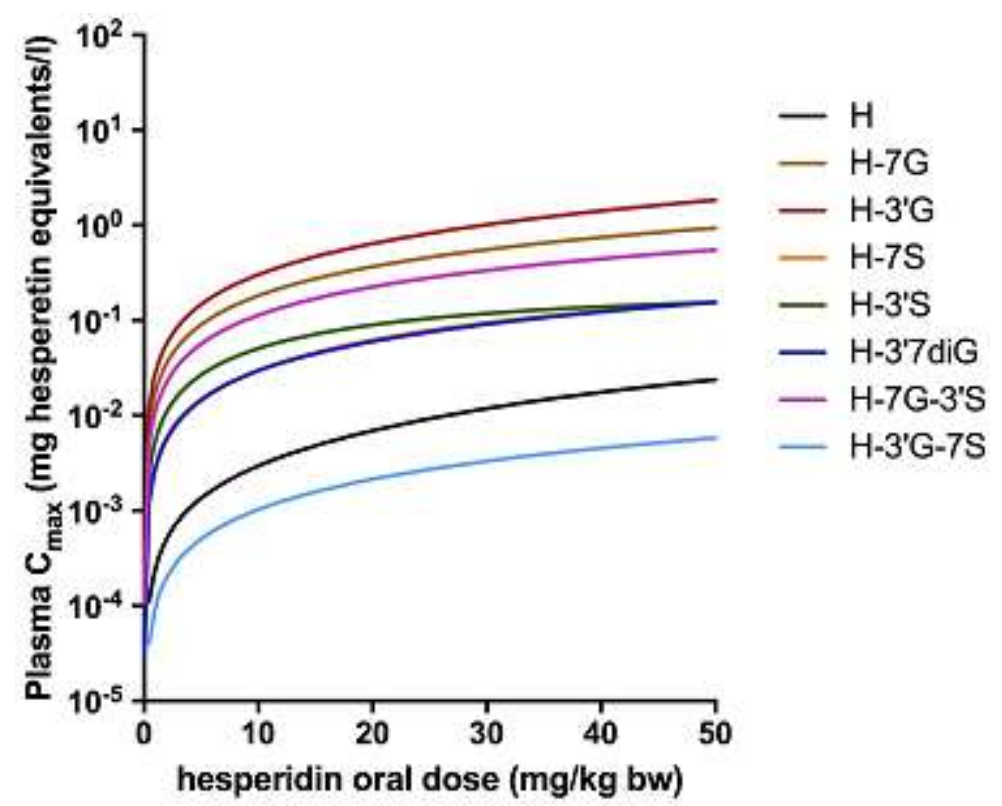

Figure 5.5 PBK model-based prediction of the dose-dependent plasma $\mathrm{C}_{\max }$ of hesperetin aglycone and its metabolites at oral doses ranging from $0.01-50 \mathrm{mg} / \mathrm{kg}$ bw hesperidin: hesperetin $(\mathrm{H})$, glucuronide (G), and sulfate (S). 
A PBK based reverse dosimetry for in vivo effective hesperidin dose levels

5.3.5 Translation of in vitro effect concentrations to in vivo effective dose levels in human

In the next step the PBK model was used for reverse dosimetry converting in vitro effective concentrations to in vivo effective dose levels of hesperidin for selected endpoints. To this end, in vitro concentration-response curves were defined in first instance for PKA inhibition by hesperetin, H-3'G, and H-7G in the present study. The results obtained reveal that hesperetin and also its major circulating metabolites, $\mathrm{H}-3^{\prime} \mathrm{G}$, and $\mathrm{H}-7 \mathrm{G}$, all inhibit PKA activity in vitro in a concentration-dependent manner as shown in the left panel of Figure 5.6A. This figure, reflecting the in vitro concentration-response curves for PKA inhibition by hesperetin, H-3'G, and H-7G (left panel of Figure 5.6A), also depicts as a first approximation the concentration-response curves for PKA inhibition by combined exposure to the mixtures of hesperetin, $\mathrm{H}-3^{\prime} \mathrm{G}$, and $\mathrm{H}-7 \mathrm{G}$, assuming the three compounds to be present at the ratios predicted to be present in plasma, and using the concentration-response curves for the individual compounds, expressing the concentration of the mixture in $\mathrm{mg}$ hesperetin equivalents/l. Figure 5.6B to 5.6D (left panels) presents similar in vitro data for the effect of hesperetin and its conjugates on other endpoints including effects on inhibition of cell migration induced by TNF- $\alpha$ (Figure 5.6B left panel), inhibition of $\mathrm{PGE}_{2}$ production induced by LPS (Figure 5.6C left panel), and inhibition of NO production induced by LPS (Figure 5.6D left panel). The latter two Figures (5.6C and 5.6D left panel) only present the in vitro concentration-response curves for the mixtures (mainly mono-conjugates) as in vitro studies for these endpoints were carried out with mixtures of hesperetin metabolites obtained from rat plasma exposed to $50 \mathrm{mg} / \mathrm{kg}$ bw hesperetin [7]. The major circulating metabolites of hesperetin in rat exposed to $50 \mathrm{mg} / \mathrm{kg}$ bw hesperidin were reported to be monoglucuronides followed by mixed conjugates containing glucuronic acid and sulfate moieties [57], which is comparable to the predicted metabolic plasma profiles in humans (Section 5.3.4). In a subsequent step, these in vitro concentration-response curves were translated into in vivo dose-response curves using a PBK model based reverse dosimetry. To this end, each in vitro mixture concentration was extrapolated to an in vivo mixtures concentration in plasma by taking into account the differences in protein and lipid concentration between the in vitro bioassays and the in vivo situations (see Section 5.2.6). The plasma in vivo concentrations of the mixtures of hesperetin and its metabolites thus obtained were used as input in the PBK model to predict the in vivo dose levels of hesperidin required to achieve these plasma concentrations, deriving the in vivo dose-response curve. Figure 5.6 (right panels) depicts the 


\section{Chapter 5}

in vivo dose-response curves obtained. The figures also present the $95 \%$ lower and upper confidence limits of the predicted in vivo dose-response curves. Subsequently, the obtained in vivo dose-response curves were used to estimate the $\mathrm{BMD}_{05}$ values for the respective biological effect of hesperidin in humans. In this way the $\mathrm{BMD}_{05}$ values for several biological effects including inhibition of PKA, inhibition of cell migration induced by TNF- $\alpha$, inhibition of $\mathrm{PGE}_{2}$ production induced by LPS, and inhibition of NO production induced by LPS, were predicted using a PBK model based reverse dosimetry.

The in vivo $\mathrm{BMD}_{05}$ values for PKA inhibition were predicted to occur at oral doses ranging from $135-539 \mathrm{mg} / \mathrm{kg}$ bw hesperidin. The in vivo $\mathrm{BMD}_{05}$ values for inhibition of endothelial cell migration were predicted to occur at oral doses ranging from $4.54-24 \mathrm{mg} / \mathrm{kg}$ bw hesperidin. The in vivo $\mathrm{BMD}_{05}$ values for inhibition of $\mathrm{PGE}_{2}$ production were predicted to occur at oral doses ranging from 2.19-4.10 mg/kg bw hesperidin. And the in vivo $\mathrm{BMD}_{05}$ values for inhibition of NO production were predicted to occur at oral doses ranging from 17$44 \mathrm{mg} / \mathrm{kg}$ bw hesperidin.

The predicted in vivo $\mathrm{BMD}_{05}$ values for effects of hesperetin and its metabolites on PKA activity, endothelial cell migration, $\mathrm{PGE}_{2}$ production, and $\mathrm{NO}$ production based on the in vitro $\mathrm{PBK}$ approach were evaluated by comparing the predicted in vivo $\mathrm{BMD}_{05}$ values with the reported active dose levels of hesperidin on anti-atherogenic and anti-inflammatory effects in healthy humans [52-54] as PKA regulates several cellular functions [21], endothelial cell migration is an initiated bioprocess for atherosclerosis [44], and $\mathrm{PGE}_{2}$ and $\mathrm{NO}$ are inflammatory biomarkers [7]. The results as shown in Figure 5.7 reveal that the predicted in vivo $\mathrm{BMD}_{05}$ values for inhibition of endothelial cell migration and $\mathrm{PGE}_{2}$ production are in line with the active dose levels of hesperidin $(4.2 \mathrm{mg} / \mathrm{kg} \mathrm{bw})$ in human intervention studies reported to result in improved endothelial function and decreased levels of inflammatory biomarkers (i.e. $\mathrm{PGE}_{2}$ and $\mathrm{CRP}$ ) [52-54]. The predicted in vivo $\mathrm{BMD}_{05}$ values for inhibition of NO production are 4- to 10-fold higher than the reported active dose levels of hesperidin in humans [52-54]. In the case of PKA activity, the predicted in vivo $\mathrm{BMD}_{05}$ values are more than 32-fold higher than the reported active dose levels of hesperidin on anti-atherogenic and anti-inflammatory effects of hesperidin in humans [52-54].

The PBK model-based predicted dose-response curves and in vivo $\mathrm{BMD}_{05}$ values derived from them also indicate that at a Western dietary and a supplementary intake of hesperidin amounting to $2.8-8$ and $7-29 \mathrm{mg} / \mathrm{kg}$ bw, respetively $[15,16]$, the plasma $\mathrm{C}_{\max }$ of total hesperetin would be high enough to inhibit endothelial cell migration, $\mathrm{PGE}_{2}$ production, and NO production (Figure 5.7). 
A. PKA activity (present study)
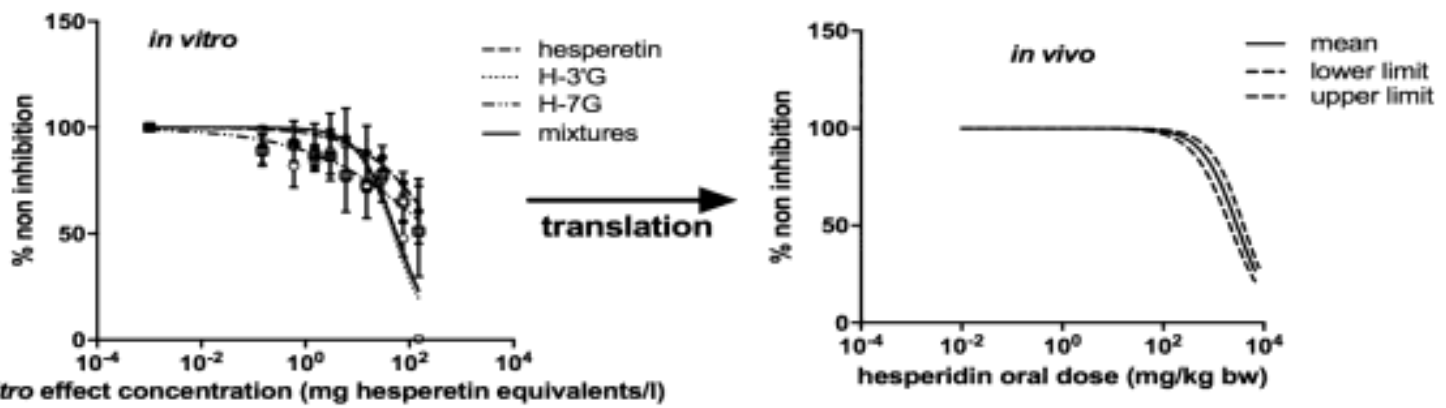

\section{B. Endothelial cell migration [44]}
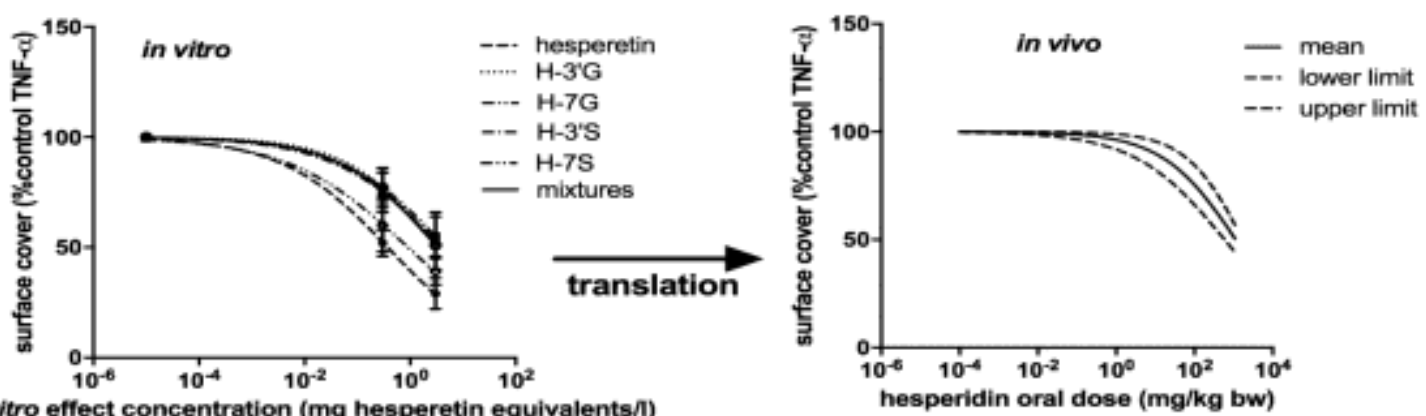

in vitro effect concentration (mg hesperetin equivalents/l)

\section{C. $\mathrm{PGE}_{2}$ production [7]}
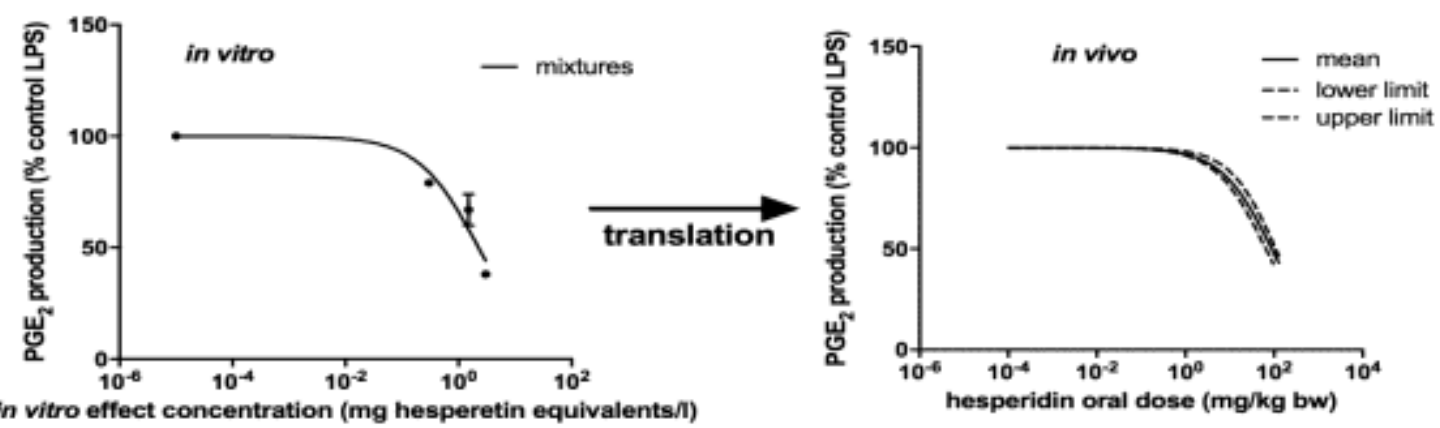

D. NO production [7]
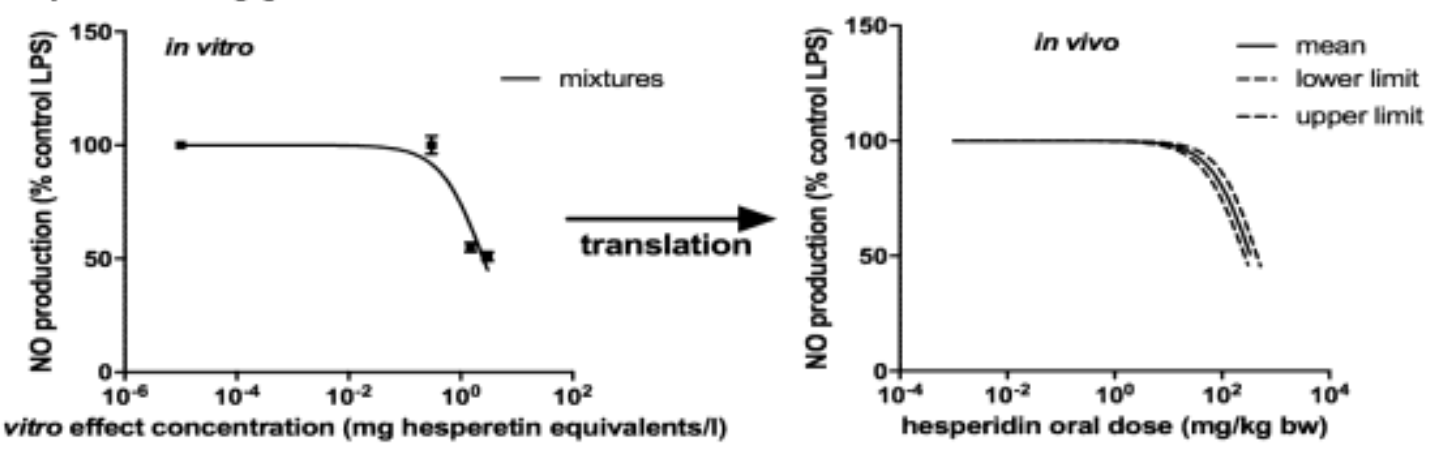

Figure 5.6 Translation of in vitro concentration-response curves for A) inhibition of PKA activity (present study), B) inhibition of TNF- $\alpha$ induced endothelial cell migration [44], C) inhibition of LPS induced prostaglandin $\mathrm{E}_{2}\left(\mathrm{PGE}_{2}\right)$ production [7], and D) inhibition of LPS induced nitric oxide (NO) production [7] to human hesperidin dose-response curves using PBK model based reverse dosimetry based on the plasma $\mathrm{C}_{\max }$ of total hesperetin (aglycone and metabolites). For inhibition of LPS induced $\mathrm{PGE}_{2}$ and NO production, in vitro studies for these endpoints were carried out with mixtures of 


\section{Chapter 5}

hesperetin metabolites, not individual metabolites, and thus only the mixtures concentration-response curves are presented (C and D left panel) [7].

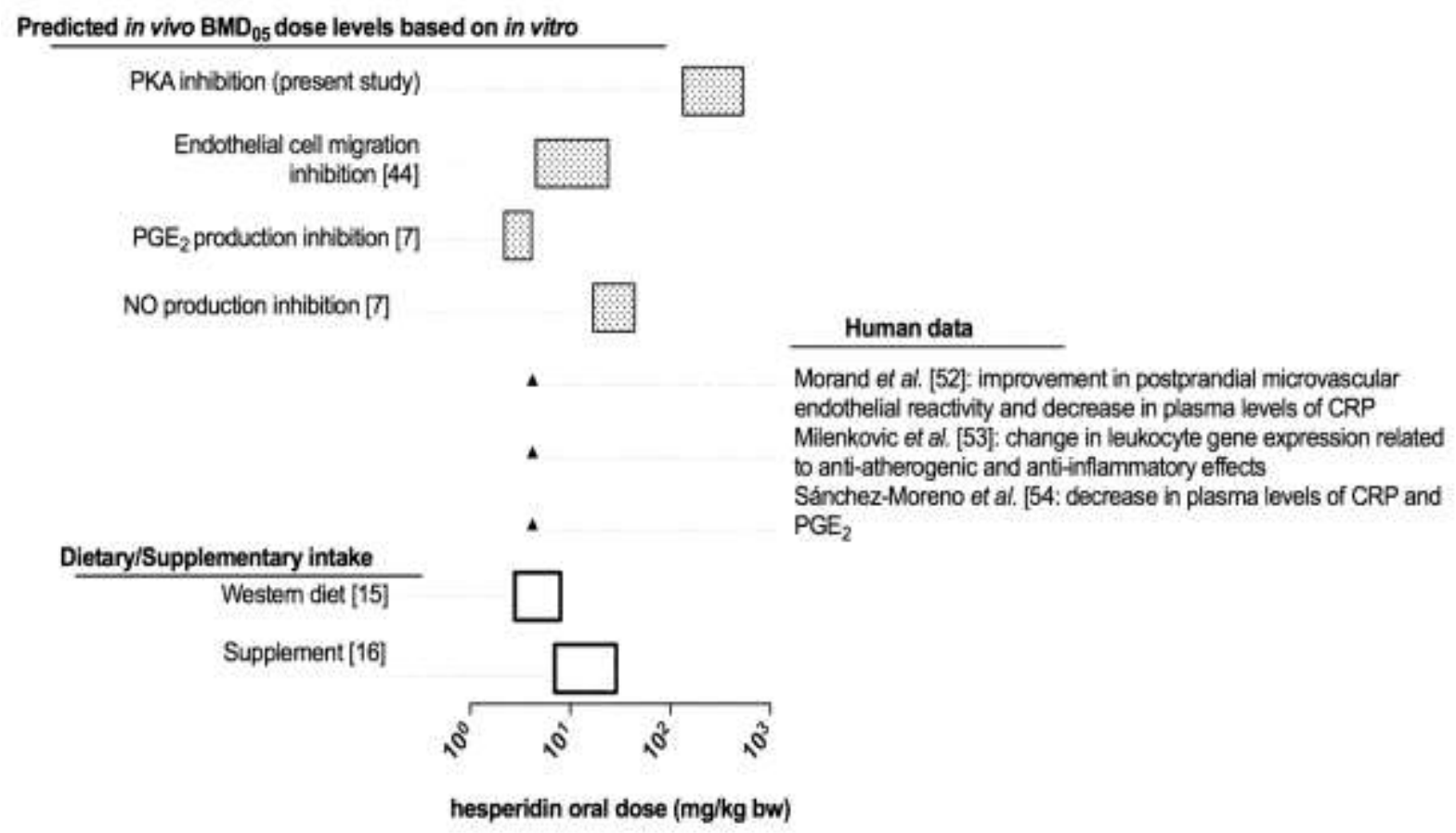

Figure 5.7 Comparison of the predicted in vivo $\mathrm{BMD}_{05}$ values of hesperidin derived from in vitro effects of the mixtures of hesperetin and its metabolites on inhibition of PKA activity (present study), endothelial cell migration [44], prostaglandin $\mathrm{E}_{2}\left(\mathrm{PGE}_{2}\right)$ production [7], and nitric oxide (NO) production [7] with the reported active dose levels on anti-atherogenic and anti-inflammatory effects of hesperidin in humans [52-54] and with a Western dietary [15] and a supplementary [16] intake of hesperidin.

\subsection{Discussion}

The aim of the present study was to develop a physiologically based kinetic (PBK) model describing the ADME of hesperidin in humans to obtain insight in dose-dependent plasma concentrations of hesperetin aglycone and its circulating metabolites and to subsequently combine the developed PBK model with in vitro effect concentrations to determine whether and if so at what oral dose levels the in vitro effects of hesperetin and its metabolites may actually occur in vivo. Evaluation of the PBK model developed against literature reported data on plasma kinetics and urinary profiles of hesperidin reveals that the model adequately predicted plasma kinetics of total hesperetin and urinary profiles of hesperidin within 1- to 7- fold deviation. The model developed was used to predict the dosedependent plasma concentrations of hesperetin aglycone as well as the type of circulating metabolites. The results revealed that the metabolic plasma profiles of hesperidin appear independent of the dose, because similar circulating metabolite patterns of hesperetin were predicted at all dose levels from 0.01 to $50 \mathrm{mg} / \mathrm{kg}$ bw hesperidin. The model predicted that $\mathrm{H}$ - 
A PBK based reverse dosimetry for in vivo effective hesperidin dose levels

$3^{\prime} \mathrm{G}$ and $\mathrm{H}-7 \mathrm{G}$ were the major circulating metabolites of hesperidin and that the plasma concentrations of hesperetin aglycone were limited and predicted to be lower than $0.02 \mathrm{mg} / \mathrm{l}$ even at an oral dose of $50 \mathrm{mg} / \mathrm{kg}$ bw hesperidin. This implies that in vivo effects of hesperidin, would probably have to be at least in part ascribed to hesperetin metabolites including $\mathrm{H}-3^{\prime} \mathrm{G}$ and $\mathrm{H}-7 \mathrm{G}$.

Given that hesperidin was predicted to be present in the systemic circulation mainly in the form of conjugated metabolites, it is crucial to understand the biological activity of these conjugates in order to gain more insight in possible in vivo effects of hesperidin. The in vitro data on PKA inhibition of hesperetin and its major circulating metabolites $\mathrm{H}-3^{\prime} \mathrm{G}$ and $\mathrm{H}-7 \mathrm{G}$ showed that the two metabolites were at least equally active in inhibition of PKA as hesperetin aglycone. Yang et al. reported that at the same concentration mixtures of hesperetin metabolites (mainly mono-conjugates) exhibited approximately 2-fold higher inhibition of $\mathrm{PGE}_{2}$ and $\mathrm{NO}$ production as compared with hesperetin aglycone in LPSstimulated RAW 264.7 [7]. Hesperetin mono-conjugates were also reported to exert inhibition activity on cell migration in TNF- $\alpha$ treated HAEC with approximately 1.5 -fold lower potency than hesperetin aglycone at the same concentration [44]. Overall, this points out that conjugation does not necessary result in loss or reduction of the biological activity and in vivo effects of hesperidin are at least in part due to its metabolites. Based on these results, it was decided that in a first approximation the concentrations of hesperetin and its metabolites could be added up for evaluation of how the in vitro effect concentrations translate into the in vivo hesperidin dose levels.

The PBK model developed provided a unique tool to gain more insight in how the in vitro concentrations translate into the in vivo dose levels of hesperidin by applying a PBK model based reverse dosimetry. The in vivo dose-response curves obtained were used to derive the $\mathrm{BMD}_{05}$ values at which plasma concentrations of hesperetin together with its metabolites would be high enough to exert 5\% response for inhibition of PKA activity, inhibition of TNF- $\alpha$ induced endothelial cell migration, inhibition of LPS induced $\mathrm{PGE}_{2}$ production, and inhibition of LPS induced NO production. The predicted in vivo $\mathrm{BMD}_{05}$ values for PKA inhibition ranged from $135-539 \mathrm{mg} / \mathrm{kg}$ bw hesperidin. The predicted in vivo $\mathrm{BMD}_{05}$ values for inhibition of TNF- $\alpha$ induced endothelial cell migration ranged from 4.54$24 \mathrm{mg} / \mathrm{kg}$ bw hesperidin. The predicted in vivo $\mathrm{BMD}_{05}$ values for inhibition of LPS induced $\mathrm{PGE}_{2}$ production ranged from 2.19-4.10 mg/kg bw hesperidin. And in the case of LPS induced NO production, the in vivo $\mathrm{BMD}_{05}$ values for inhibition ranged from $17-44 \mathrm{mg} / \mathrm{kg}$ bw 


\section{Chapter 5}

hesperidin. These predictions were evaluated against the reported active dose levels of hesperidin for anti-atherogenic and anti-inflammatory effect as reported in humans [52-54]. The predicted in vivo $\mathrm{BMD}_{05}$ values for inhibition of endothelial cell migration and $\mathrm{PGE}_{2}$ production are in line with the reported active dose levels of hesperidin in humans for improving endothelial function and decreasing levels of inflammatory biomarkers (i.e. $\mathrm{PGE}_{2}$ and CRP) [52-54]. The predicted in vivo $\mathrm{BMD}_{05}$ values for inhibition of NO production are 4to 10-fold higher than the reported active dose levels of hesperidin in humans [52-54]. The predicted in vivo $\mathrm{BMD}_{05}$ values for PKA inhibition are more than 32-fold higher than the reported active dose levels for anti-atherogenic and anti-inflammatory effects of hesperidin in humans [52-54]. This might imply that inhibition of PKA activity (a regulator of several cellular functions [21]) is not a major mechanism for anti-atherogenic and anti-inflammatory action of hesperidin in vivo.

Based on the predicted in vivo $\mathrm{BMD}_{05}$ values for hesperidin derived from in vitro concentration-response curves for hesperidin mediated inhibition of PKA activity, TNF- $\alpha$ induced endothelial cell migration, and LPS induced production of $\mathrm{PGE}_{2}$ and $\mathrm{NO}$, it can also be concluded that the plasma $\mathrm{C}_{\max }$ of total hesperetin (aglycone and metabolites) at a Western dietary and a supplementary intake amounting to 2.8-8 [15] and 7-29 [16] $\mathrm{mg} / \mathrm{kg}$ bw hesperidin, would be high enough to induce these effects on endothelial cell migration and pro-inflammatory molecules in vivo. For the inhibition of PKA activity, the plasma $\mathrm{C}_{\max }$ of total hesperetin upon these dietary or supplementary intakes would generally not be high enough to induce a similar effect on PKA activity in vivo.

Overall, the PBK model developed in the present study adequately predicted ADME of hesperidin in humans. The model obtained was also shown to be of use to translate in vitro results on the biological effects of hesperetin aglycone and its metabolites to the human in vivo situation and evaluate the possible occurrence of in vivo effects without the need for human intervention studies.

\section{Conflict of interest}

The authors state no conflict of interest.

\section{Acknowledgement}

This research was financially supported by the Ministry of Science and Technology of Thailand through a Royal Thai Government Scholarship awarded to Rungnapa Boonpawa for conducting her $\mathrm{PhD}$ in The Netherlands. 
A PBK based reverse dosimetry for in vivo effective hesperidin dose levels

\section{References}

1. Pereira-Caro, G., et al., Orange juice (poly)phenols are highly bioavailable in humans. The American Journal of Clinical Nutrition, 2014. 100(5): p. 1378-1384.

2. Tomás-Barberán, F. A. and M. N. Clifford, Flavanones, chalcones and dihydrochalcones nature, occurrence and dietary burden. Journal of the Science of Food and Agriculture, 2000. 80(7): p. 1073-1080.

3. Roohbakhsh, A., et al., Molecular mechanisms behind the biological effects of hesperidin and hesperetin for the prevention of cancer and cardiovascular diseases. Life Sciences, 2015. 124: p. 64-74.

4. Smina, T. P., et al., Hesperetin exerts apoptotic effect on A431 skin carcinoma cells by regulating mitogen activated protein kinases and cyclins Cellular \& Molecular Biology, 2015. 61(6): p. 92-99.

5. Choi, E. J., Hesperetin induced G1-phase cell cycle arrest in human breast cancer MCF-7 cells: involvement of CDK4 and p21. Nutrition and Cancer, 2007. 59(1): p. 115-119.

6. Palit, S., et al., Hesperetin induces apoptosis in breast carcinoma by triggering accumulation of ROS and activation of ASK1/JNK pathway. Journal of Cellular Physiology, 2015. 230(8): p. 1729-1739.

7. Yang, H.-L., et al., Antioxidant and anti-inflammatory potential of hesperetin metabolites obtained from hesperetin-administered rat serum: an ex vivo approach. Journal of Agricultural and Food Chemistry, 2012. 60(1): p. 522-532.

8. Chanet, A., et al., Flavanone metabolites decrease monocyte adhesion to TNF- $\alpha$-activated endothelial cells by modulating expression of atherosclerosis-related genes. British Journal of Nutrition, 2013. 110(04): p. 587-598.

9. Kim, G. D., Hesperetin inhibits vascular formation by suppressing of the PI3K/AKT, ERK, and p38 MAPK signaling pathways. Preventive Nutrition and Food Science, 2014. 19(4): p. 299-306.

10. Trzeciakiewicz, A., et al., Molecular mechanism of hesperetin-7-O-glucuronide, the main circulating metabolite of hesperidin, involved in osteoblast differentiation. Journal of Agricultural and Food Chemistry, 2009. 58(1): p. 668-675.

11. Mullen, W., et al., Bioavailability and metabolism of orange juice flavanones in humans: impact of a full-fat yogurt. Journal of Agricultural and Food Chemistry, 2008. 56(23): p. 11157-11164.

12. Takumi, H., et al., Bioavailability of orally administered water-dispersible hesperetin and its effect on peripheral vasodilatation in human subjects: implication of endothelial functions of plasma conjugated metabolites. Food \& Function, 2012. 3(4): p. 389-398.

13. Brett, G. M., et al., Absorption, metabolism and excretion of flavanones from single portions of orange fruit and juice and effects of anthropometric variables and contraceptive pill use on flavanone excretion. British Journal of Nutrition, 2009. 101(05): p. 664-675.

14. Manach, C., et al., Bioavailability in humans of the flavanones hesperidin and narirutin after the ingestion of two doses of orange juice. European Journal of Clinical Nutrition, 2003. 57(2): p. 235-242.

15. Koch, W., et al., Application of TLC method with video scanning in estimation of daily dietary intake of specific flavonoids-preliminary studies. Acta Poloniae Pharmaceutica, 2013. 70(4): p. 611-620.

16. Hendler, S. S. and D. M. Rorvik, PDR for nutritional supplements. 2nd ed. 2008, New Jersey: Thomson Reuters. 
17. Brand, W., et al., Phase II Metabolism of hesperetin by individual UDPglucuronosyltransferases and sulfotransferases and rat and human tissue samples. Drug Metabolism and Disposition, 2010. 38(4): p. 617-625.

18. Perche, O., et al., Orange juice and its major polyphenol hesperidin consumption do not induce immunomodulation in healthy well-nourished humans. Clinical Nutrition, 2013. 33(1): p. 130-135.

19. Oleson, L. and M. H. Court, Effect of the $\beta$-glucuronidase inhibitor saccharolactone on glucuronidation by human tissue microsomes and recombinant UDPglucuronosyltransferases (UGTs). The Journal of pharmacy and pharmacology, 2008. 60(9): p. 1175-1182.

20. Wright, B., et al., GRID and docking analyses reveal a molecular basis for flavonoid inhibition of Src family kinase activity. The Journal of Nutritional Biochemistry, 2015. 26(11): p. 1156-1165.

21. Howe, A. K., Regulation of actin-based cell migration by cAMP/PKA. Biochimica et Biophysica Acta (BBA) - Molecular Cell Research, 2004. 1692(2-3): p. 159-174.

22. Chen, N., et al., Determination of kinetic parameters and structure-activity relationships of ginsenosides as inhibitors of cyclin-dependent kinase 5/p25 using ultra-pressure liquid chromatography with triple quadrupole tandem mass spectrometry. Rapid Communications in Mass Spectrometry, 2013. 27(9): p. 985-992.

23. Meggio, F., et al., Different susceptibility of protein kinases to staurosporine inhibition. European Journal of Biochemistry, 1995. 234(1): p. 317-322.

24. Boonpawa, R., et al., A physiologically based kinetic (PBK) model describing plasma concentrations of quercetin and its metabolites in rats. Biochemical Pharmacology, 2014. 89(2): p. 287-299.

25. Boonpawa, R., et al., Use of physiologically based kinetic (PBK) modeling to study interindividual human variation and species differences in plasma concentrations of quercetin and its metabolites. Biochemical Pharmacology, 2015. 98(4): p. 690-702.

26. Erlund, I., et al., Plasma kinetics and urinary excretion of the flavanones naringenin and hesperetin in humans after ingestion of orange juice and grapefruit juice. The Journal of Nutrition, 2001. 131(2): p. 235-241.

27. Nielsen, I. L. F., et al., Bioavailability is improved by enzymatic modification of the citrus flavonoid hesperidin in humans: a randomized, double-blind, crossover trial. The Journal of Nutrition, 2006. 136(2): p. 404-408.

28. Gardana, C., et al., Flavanone plasma pharmacokinetics from blood orange juice in human subjects. British Journal of Nutrition, 2007. 98(01): p. 165-172.

29. Silberberg, M., et al., The bioavailability of polyphenols is highly governed by the capacity of the intestine and of the liver to secrete conjugated metabolites. European Journal of Nutrition, 2006. 45(2): p. 88-96.

30. Steensma, A., H. P. J. M. Noteborn, and H. A. Kuiper, Comparison of Caco-2, IEC-18 and HCEC cell lines as a model for intestinal absorption of genistein, daidzein and their glycosides. Environmental Toxicology and Pharmacology, 2004. 16(3): p. 131-139.

31. Kimura, T. and K. Higaki, Gastrointestinal transit and drug absorption. Biological and Pharmaceutical Bulletin, 2002. 25(2): p. 149-164.

32. Cubitt, H., J. B. Houston, and A. Galetin, Relative importance of intestinal and hepatic glucuronidation -impact on the prediction of drug clearance. Pharmaceutical Research, 2009. 26(5): p. 1073-1083. 
A PBK based reverse dosimetry for in vivo effective hesperidin dose levels

33. Cubitt, H. E., J. B. Houston, and A. Galetin, Prediction of human drug clearance by multiple metabolic pathways: integration of hepatic and intestinal microsomal and cytosolic data. Drug Metabolism and Disposition, 2011. 39(5): p. 864-873.

34. van de Kerkhof, E. G., I. A. M. de Graaf, and G. M. M. Groothuis, In vitro methods to study intestinal drug metabolism. Current Drug Metabolism, 2007. 8(7): p. 658-675.

35. Malfatti, M. A., et al., The capability of rat colon tissue slices to metabolize the cooked-food carcinogen 2-amino-1-methyl-6-phenylimidazo[4,5-b]pyridine. Cancer Research, 1996. 56(11): p. 2550-2555.

36. Brown, R. P., et al., Physiological parameter values for physiologically based pharmacokinetic models. Toxicology and Industrial Health, 1997. 13(4): p. 407-484.

37. DeJongh, J., H. J. M. Verhaar, and J. L. M. Hermens, A quantitative property-property relationship (QPPR) approach to estimate in vitro tissue-blood partition coefficients of organic chemicals in rats and humans. Archives of Toxicology, 1997. 72(1): p. 17-25.

38. Simcyp. Simcyp prediction tools -fu. [Online]. Available from: https://members.simcyp.com/account/tools/fu/). [Accessed: 15th July 2015]. 2014 [cited 2014.

39. Simcyp. Simcyp prediction tools- blood to plasma partition ratio (B/P). [Online]. Available from: https://members.simcyp.com/account/tools/BP/). [Accessed: 15th July 2015]. 2014.

40. Bredsdorff, L., et al., Absorption, conjugation and excretion of the flavanones, naringenin and hesperetin from $\alpha$-rhamnosidase-treated orange juice in human subjects. British Journal of Nutrition, 2010. 103(11): p. 1602-1609.

41. Evans, M. V. and M. E. Andersen, Sensitivity analysis of a physiological model for 2,3,7,8Tetrachlorodibenzo-p-dioxin (TCDD): Assessing the impact of specific model parameters on sequestration in liver and fat in the rat. Toxicological Sciences, 2000. 54(1): p. 71-80.

42. Louisse, J., et al., Prediction of in vivo developmental toxicity of all-trans-retinoic acid based on in vitro toxicity data and in silico physiologically based kinetic modeling. Archives of Toxicology, 2015. 89(7): p. 1135-1148.

43. Strikwold, M., et al., Combining in vitro embryotoxicity data with physiologically based kinetic (PBK) modelling to define in vivo dose-response curves for developmental toxicity of phenol in rat and human. Archives of Toxicology, 2013. 87(9): p. 1709-1723.

44. Gimenez-Bastida, J. A., et al., Hesperetin and its sulfate and glucuronide metabolites inhibit TNF- $\alpha$ induced human aortic endothelial cell migration and decrease plasminogen activator inhibitor-1 (PAI-1) levels. Food \& Function, 2016. 7(1): p. 118-126.

45. Kortenkamp, A., T. Backhaus, and M. Faust, State of the art on mixture toxicity. Report. Available

at: http://ec.europa.eu/environment/chemicals/effects/pdf/report_mixture_toxicity.pdf. $\quad$ Last accessed 3 August 2016. 2009.

46. Gülden, M. and H. Seibert, In vitro-in vivo extrapolation: estimation of human serum concentrations of chemicals equivalent to cytotoxic concentrations in vitro. Toxicology, 2003. 189(3): p. 211-222.

47. Gülden, M., et al., Impact of protein binding on the availability and cytotoxic potency of organochlorine pesticides and chlorophenols in vitro. Toxicology, 2002. 175(1-3): p. 201213.

48. Khan, M. K., et al., Binding of citrus flavanones and their glucuronides and chalcones to human serum albumin. Food \& Function, 2011. 2(10): p. 617-626.

49. Xie, M.-X., X.-Y. Xu, and Y.-D. Wang, Interaction between hesperetin and human serum albumin revealed by spectroscopic methods. Biochimica et Biophysica Acta (BBA) - General Subjects, 2005. 1724(1-2): p. 215-224. 


\section{Chapter 5}

50. Mahesha, H. G., et al., A spectroscopic study of the interaction of isoflavones with human serum albumin. FEBS Journal, 2006. 273(3): p. 451-467.

51. EFSA, Guidance of the Scientific Committee on a request from EFSA on the use of the benchmark dose approach in risk assessment. The EFSA Journal, 2009. 1150: p. 1-72.

52. Morand, C., et al., Hesperidin contributes to the vascular protective effects of orange juice: a randomized crossover study in healthy volunteers. Am J ClinNutr, 2011. 93.

53. Milenkovic, D., et al., Hesperidin displays relevant role in the nutrigenomic effect of orange juice on blood leukocytes in human volunteers: a randomized controlled cross-over study. PLoS ONE, 2011. 6(11): p. e26669.

54. Sánchez-Moreno, C., et al., High-pressurized orange juice consumption affects plasma vitamin $C$, antioxidative status and inflammatory markers in healthy humans. The Journal of Nutrition, 2003. 133(7): p. 2204-2209.

55. Argikar, U. A., Unusual Glucuronides. Drug Metabolism and Disposition, 2012. 40(7): p. 1239-1251.

56. Boersma, M. G., et al., Regioselectivity of phase II metabolism of luteolin and quercetin by UDP-glucuronosyl transferases. Chemical Research in Toxicology, 2002. 15(5): p. 662-670.

57. Matsumoto, H., et al., Identification and quantification of the conjugated metabolites derived from orally administered hesperidin in rat plasma. Journal of Agricultural and Food Chemistry, 2004. 52(21): p. 6653-6659. 
A PBK based reverse dosimetry for in vivo effective hesperidin dose levels

\section{Supporting information}
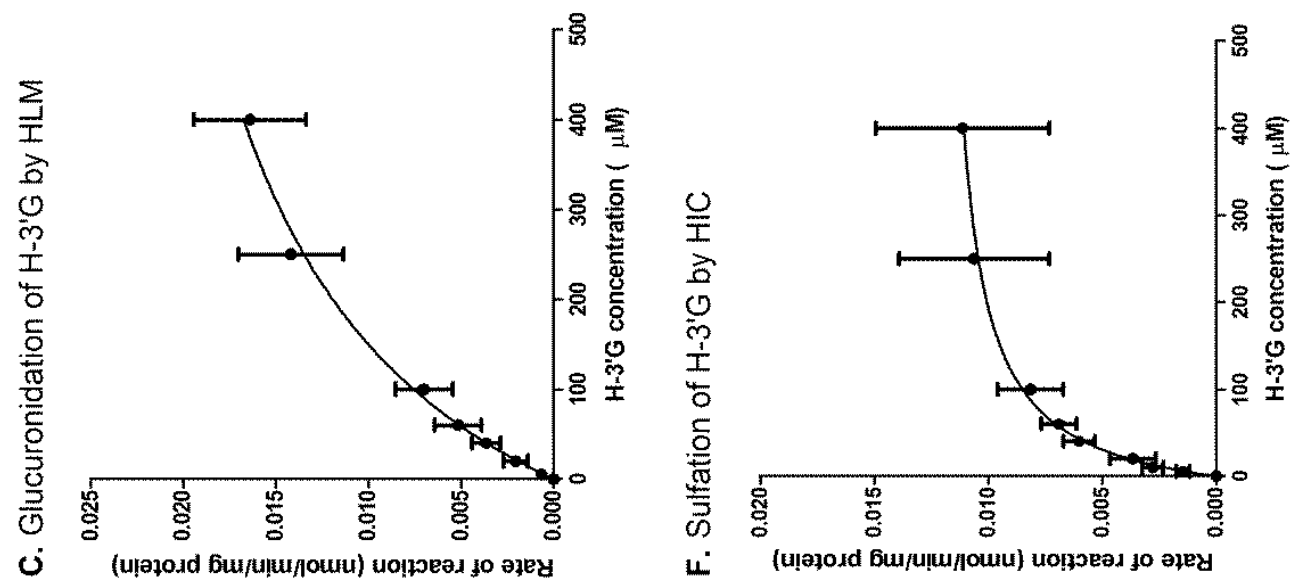

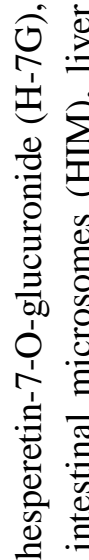

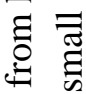

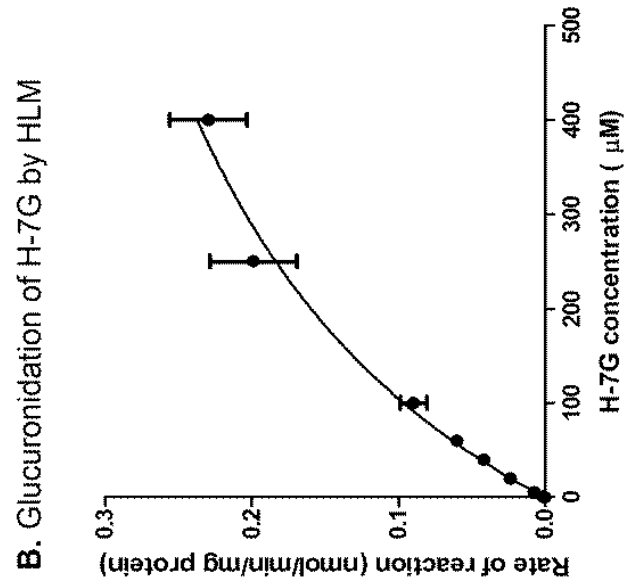

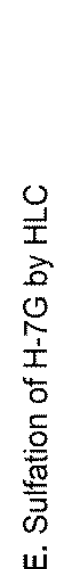

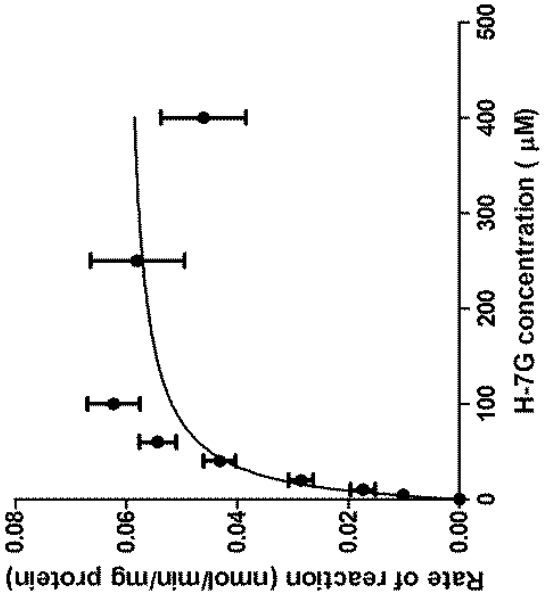

बै

茠

帚寻 寻

$=\frac{1}{0}$

I

式

要

च ठे

पै

융

휴을

응

in
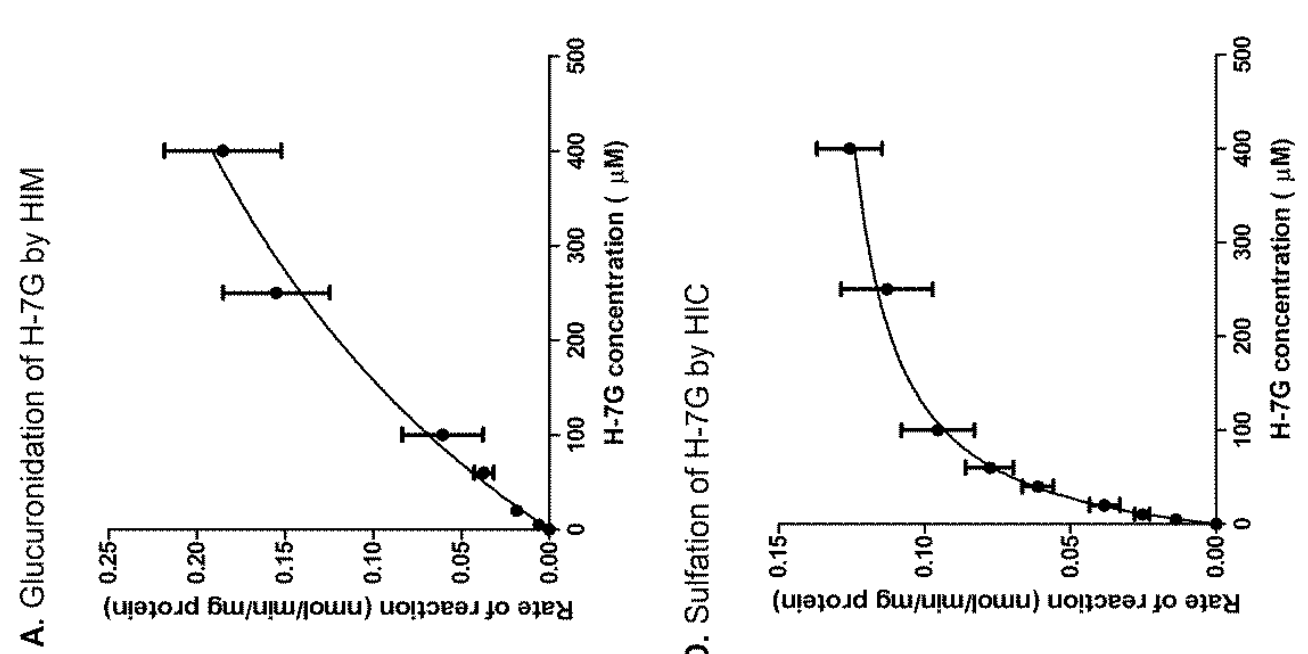

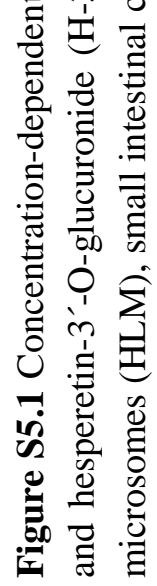


Chapter 5
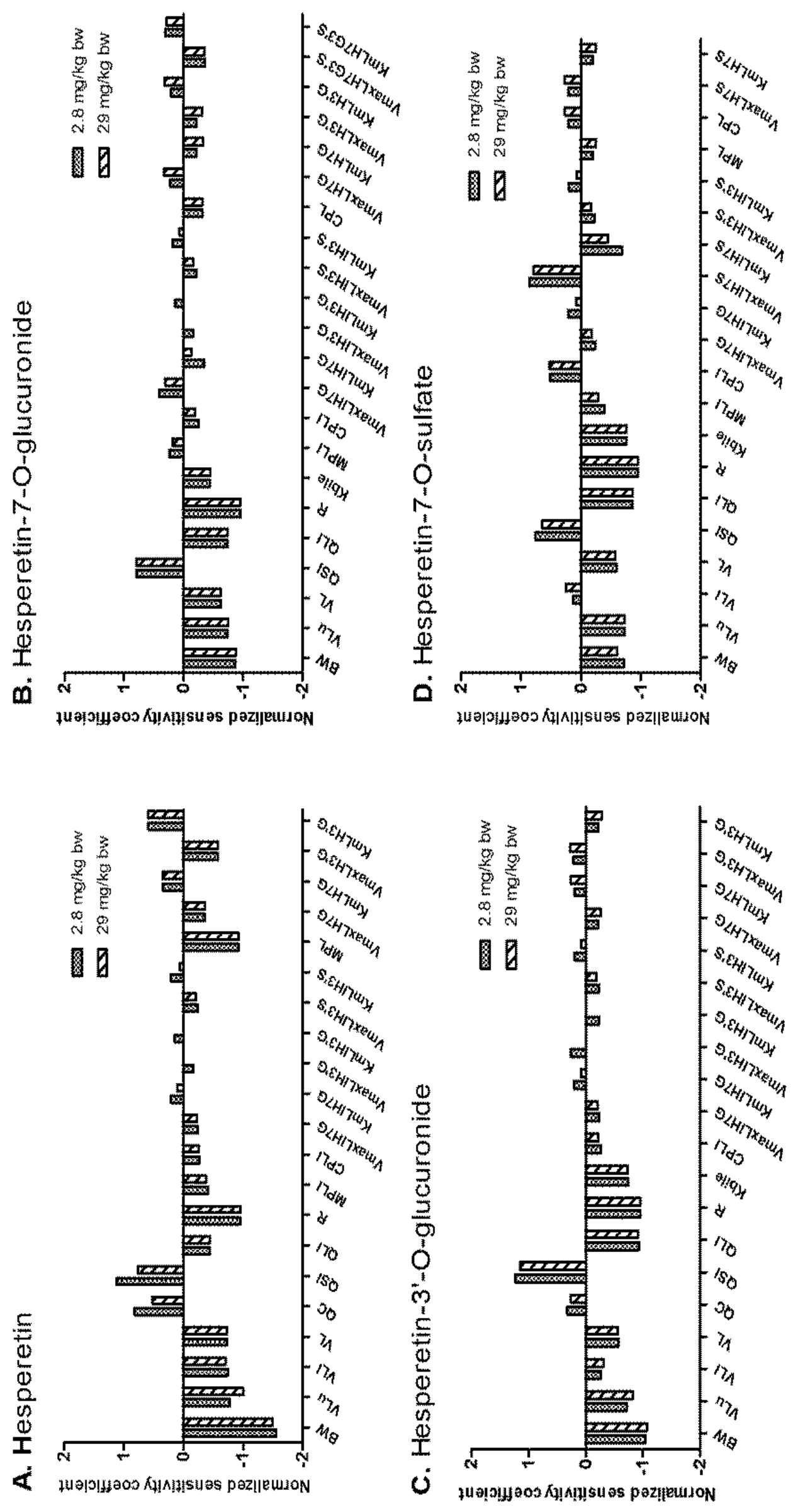

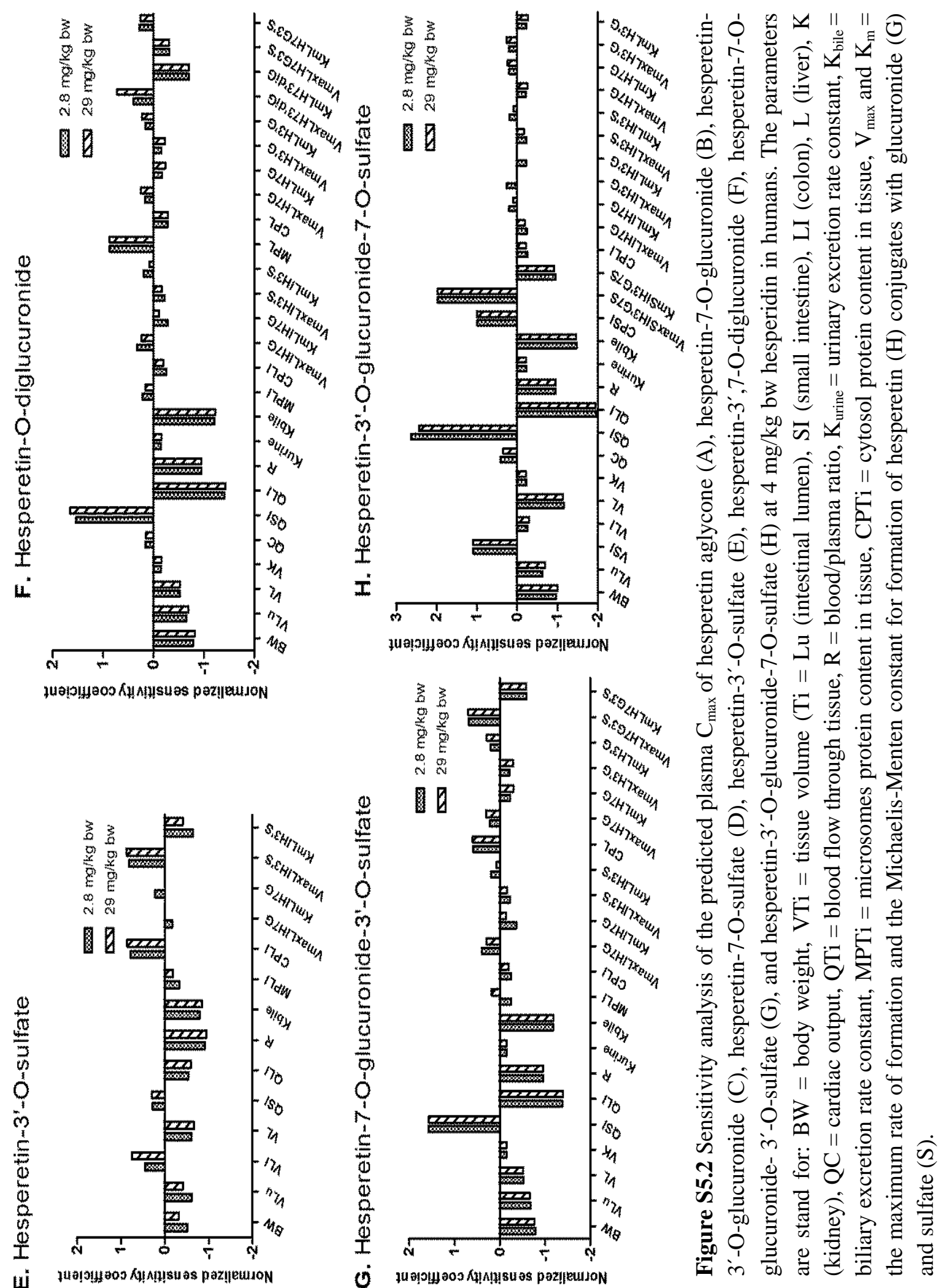
Chapter 5 


\section{CHAPTER 6}

General discussion and future perspectives 


\subsection{Introduction and main findings}

Flavonoids are polyphenolic compounds abundantly present in fruits and vegetables mainly as $\beta$-glycosides [1]. Flavonoids have been proposed to exert beneficial health effects against various degenerative conditions including cancer [2], cardiovascular diseases [3], obesity and diabetes [4, 5], as well as neurodegenerative disorders [6, 7]. Possible modes of action of flavonoids underlying these beneficial health effects include their activity as ligands for various receptors, kinases, enzymes, and/or transport proteins, and these activities have often been studied using in vitro test systems with high concentrations of the flavonoid aglycones [8-10]. However, the question has been raised on how relevant these in vitro results are for the in vivo situation as bioavailability of flavonoid aglycones in vivo would be limited [9]. Upon ingestion, flavonoid glycosides are deconjugated and upon absorption from the gastrointestinal tract flavonoid aglycones are extensively metabolized, mainly by UDPglucuronosyl transferases (UGTs), sulfotransferases (SULTs), and catechol Omethyltransferases (COMTs) in both intestine and liver cells [11]. As a result, flavonoids are mainly present in their conjugated forms in the systemic circulation [12].

Conjugation of flavonoids may affect their biological activities depending on the type and position of the conjugation [10]. To better understand in vivo effects of flavonoids, it is of importance to gain insight in absorption, distribution, metabolism, and excretion (ADME) of flavonoids in vivo. Therefore, the aim of the present $\mathrm{PhD}$ project was to gain quantitative insight in dose-dependent plasma concentrations of flavonoid aglycones and their metabolites using physiologically based kinetic (PBK) modeling and to use these PBK models to contribute to the better understanding of whether the in vitro effect concentrations are relevant for the in vivo situation upon realistic dose levels and thus whether these dose levels would actually result in biological effects of flavonoids in vivo. The present $\mathrm{PhD}$ project focused on the flavonoids quercetin, genistein and hesperetin as model compounds.

In Chapter 2, a PBK model was built for quercetin and its glycoside quercetin-4'-Oglucoside to predict dose-dependent plasma concentration of quercetin aglycone and to tentatively identify the nature and the conjugation position and concentrations of the circulating metabolites in rats. Model development was performed by integrating kinetic parameters obtained from in vitro incubations with relevant rat tissue fractions and by fitting kinetic parameters to literature available in vivo data. The model adequately predicted plasma concentrations of different metabolites of quercetin within 1.9- to 3-fold deviation as compared with literature reported plasma concentrations of these metabolites in rats exposed to quercetin- 
4'-O-glucoside. The model predictions revealed that bioavailability of quercetin aglycone is very low ranging from $0.004-0.1 \%$ of the dose at different oral doses of quercetin or quercetin4'-O-glucoside. Glucuronidation appeared to be the major pathway responsible for the limited bioavailability of quercetin aglycone, with $95-99 \%$ of the dose predicted to be converted to monoglucuronides within 1.5-2.5 h. Due to the fast metabolic conversion of monoglucuronides, these metabolites were subjected to further conjugation forming di- and tri-conjugates, and these were predicted to be the major circulating forms of quercetin in rats. The major circulating metabolites of quercetin in rats, at normal dietary estimated daily human intake levels, were tentatively identified to be quercetin-7-O-glucuronide-3'-O-sulfate-4'-O-methylate, followed by quercetin-7, 4'-O-diglucuronide, and quercetin-7-O-glucuronide-3'-0-sulfate.

In Chapter 3, the PBK models for quercetin and quercetin-4'-O-glucoside developed in rats were used as a platform to build a PBK model for quercetin in humans in order to define species differences in ADME of quercetin between humans and rats. First, a PBK model for an average human was developed, which was subsequently extended to 20 different individual human PBK models to assess interindividual human variation. The kinetic parameters for metabolic conversion of quercetin were obtained from in vitro incubations with pooled (for the average human model) and 20 individual human tissue fractions (for individual human models). The models adequately predicted metabolic profiles of quercetin in plasma and urine within 1to 7-fold deviation from reported data in humans. The PBK model predicted that quercetin-3'O-glucuronide is the major circulating metabolite in 19 out of the 20 individuals, indicating limited interindividual human variation. Significant species differences occurred in the major circulating metabolites as quercetin was predicted to be mainly present as mono-glucuronides in human plasma but as di- and tri-conjugates in rat plasma, suggesting that rat may not be an adequate model to study effects of quercetin in humans.

For many flavonoids other than quercetin, in vivo kinetic data are still lacking, and therefore the developed PBK models for quercetin formed a basis for the development of similar models for other relevant dietary flavonoids such as genistein (soy-based isoflavone) and hesperetin (citrus-based flavanone) in Chapter 4 and Chapter 5, respectively. Chapter 4 describes the development of a PBK model for genistein and its glycoside genistin to gain insight in dose-dependent plasma concentrations of genistein aglycone and its metabolites in humans. Given that the in vivo estrogenic responses of the soy isoflavones genistein and genistin are still under debate, the PBK model obtained for genistein and genistin was used to facilitate the translation of in vitro estrogenic concentration-response curves reported in 


\section{Chapter 6}

literature to in vivo dose-response curves by applying PBK model based reverse dosimetry. It is of importance to note that especially genistein aglycone, and not its metabolites, is likely responsible for estrogenic responses in vivo as genistein conjugates tested so far, including genistein-7-O-glucuronide, genistein-7-O-sulfate, and genistein-4'-O-sulfate are inactive in assays for estrogenicity $[13,14]$. The developed PBK model adequately predicted metabolic profiles of genistein in plasma and urine within 1- to 7-fold deviation from the reported data in humans. The PBK model predicted that genistein-7-O-glucuronide is the major circulating metabolite of genistein in human plasma and that plasma concentrations of genistein aglycone are generally low, amounting to $0.5-17 \%$ of total plasma genistein (aglycone and metabolites) at oral doses ranging from $0.01-50 \mathrm{mg} / \mathrm{kg}$ bw genistein. When consumed as the glycoside genistin, similar metabolic profiles of genistein in plasma were predicted, requiring approximately 1.3-fold higher oral dose levels (expressed in genistein equivalents) to achieve similar plasma concentrations. This can be explained by the slow release of genistein aglycone from its glycoside genistin that limits the amount of genistein being taken up resulting in lower genistein concentrations in plasma and tissues at equimolar dose levels. Using the PBK model obtained to convert in vitro concentration-response curves for estrogenic activity of genistein to in vivo dose-response curves, in vivo benchmark dose for $5 \%$ response $\left(\mathrm{BMD}_{05}\right)$ values for estrogenicity between $0.03-4.56 \mathrm{mg} / \mathrm{kg}$ bw genistein were predicted. For genistin, these values were 1.3-fold higher due to the slow release of genistein aglycone from its glycoside genistin that limits the amount of genistein being taken up resulting in lower genistein concentrations in the plasma. These values are in line with the reported in vivo dose levels of genistein that appear to induce estrogenic responses in humans. The predicted in vivo $\mathrm{BMD}_{05}$ values for genistein estrogenicity also revealed that at an Asian dietary and a supplementary intake of genistein amounting to $0.21-0.71$ [15] and 0.43-13 [16] mg/kg bw estrogenic responses can be expected, while intake resulting from a Western diet amounting to $0.003-0.01 \mathrm{mg} / \mathrm{kg}$ bw [16] may not be effective. Overall, this study shows how plasma concentrations of genistein and its metabolites and estrogenic dose levels of genistein in humans can be predicted by combining in vitro concentration-response curves with PBK model based reverse dosimetry, eliminating the need for human intervention studies.

In Chapter 5, the PBK models for quercetin were further used as a platform to build a PBK model for hesperidin, a glycoside and natural existing form of the flavanone hesperetin known to be present in citrus fruits, to gain more insight in dose-dependent plasma concentrations of hesperetin aglycone and its metabolites in humans. Also in this case, the PBK model obtained was used to facilitate translation of in vitro concentration-response curves for 
effects of hesperetin and its metabolites on protein kinase A (PKA) activity and other biological activities including effects on TNF- $\alpha$ induced endothelial cell migration, LPS induced prostaglandin $\mathrm{E}_{2}\left(\mathrm{PGE}_{2}\right)$ production, and LPS induced nitric oxide (NO) production available in literature, to in vivo dose-response curves of hesperidin in humans by applying PBK-model based reverse dosimetry. The PBK model obtained adequately predicted plasma kinetics of total hesperetin (aglycone and metabolites) and also the amount and nature of different hesperetin metabolites excreted in urine within 1- to 7-fold deviation from the reported data in humans. The PBK model predicted that hesperetin-3'-O-glucuronide and hesperetin-7-O-glucuronide are the major circulating metabolites of hesperidin in humans accounting for approximately $70 \%$ of the total plasma hesperetin (expressed in $\mathrm{mg}$ hesperetin equivalents/l) and that plasma concentrations of hesperetin aglycone are lower than $0.02 \mathrm{mg} / \mathrm{l}$ even at a high oral dose of 50 $\mathrm{mg} / \mathrm{kg}$ bw hesperidin. The PBK model thus obtained was used to convert available in vitro concentration-response curves for activity of hesperetin together with its metabolites to in vivo dose-response curves from which subsequently $\mathrm{BMD}_{05}$ values were derived. The in vivo $\mathrm{BMD}_{05}$ values for PKA inhibition by hesperetin and its monoglucuronides based on the results from an in vitro PKA inhibition assay were predicted to amount to $135-539 \mathrm{mg} / \mathrm{kg}$ bw hesperidin. The in vivo $\mathrm{BMD}_{05}$ values for inhibition of TNF- $\alpha$ induced endothelial cell migration derived from in vitro data were predicted to amount to $4.54-24 \mathrm{mg} / \mathrm{kg}$ bw hesperidin. The in vivo $\mathrm{BMD}_{05}$ values for inhibition of LPS induced $\mathrm{PGE}_{2}$ production derived from in vitro data were predicted to range from 2.19-4.10 mg/kg bw hesperidin. The in vivo $\mathrm{BMD}_{05}$ values for inhibition of LPS induced NO production derived from in vitro data were predicted to vary from $17-44 \mathrm{mg} / \mathrm{kg}$ bw hesperidin. These predicted in vivo $\mathrm{BMD}_{05}$ values were compared to the reported active dose levels on anti-atherogenic and anti-inflammatory effects of hesperidin in humans [17-19]. The results obtained revealed that only the predicted in vivo $\mathrm{BMD}_{05}$ values for inhibition of endothelial cell migration and $\mathrm{PGE}_{2}$ production fell within the reported active dose levels of hesperidin $(4.2 \mathrm{mg} / \mathrm{kg} \mathrm{bw})$ in humans resulting in improved endothelial function and decreased levels of inflammatory biomarkers (i.e. PGE $_{2}$ and C-reactive protein) [17-19]. The predicted in vivo $\mathrm{BMD}_{05}$ values for inhibition of LPS induced NO production are 4- to 10-fold higher than the reported active dose levels of hesperidin in humans [17-19], which could be considered acceptable as within one order of magnitude [20]. In the case of PKA activity, the predicted in vivo $\mathrm{BMD}_{05}$ values are more than 32-fold higher than the reported active dose levels of hesperidin on endothelial function in humans $[17,18]$. This might indicate that inhibition of PKA activity may not represent a major mechanism involved in endothelial function. The 


\section{Chapter 6}

predicted in vivo $\mathrm{BMD}_{05}$ values also revealed that inhibition of endothelial cell migration and $\mathrm{PGE}_{2}$ and NO production can be expected at a Western dietary and a supplementary intake of hesperidin. In conclusion, the PBK model obtained adequately predicted ADME of hesperidin in humans and was shown to be of use to translate in vitro results to the human in vivo situation without the need for human intervention studies.

\subsection{General discussion and future perspectives}

The PBK models developed in the present thesis have a great potential to facilitate further understanding of in vivo effects for a wider range of flavonoids, for which in vivo data are limited. The following sections discuss whether and how the results obtained in the present thesis can contribute to future research on flavonoids focusing on:

- further refinement of the PBK models,

- in vitro biological studies of flavonoid mediated effects, and

- use of PBK model based reverse dosimetry.

\subsubsection{Further refinement of the PBK models}

In the next sections selected topics for further future refinement and improvement of the PBK models are discussed in some more detail.

\section{Defining excretion parameters for the PBK models}

Excretion parameters are influential parameters that affect plasma concentrations of flavonoid metabolites as also reflected by the sensitivity analyses for plasma concentrations of quercetin, genistein, and hesperetin metabolites (expressing normalized sensitivity coefficients higher than 0.1 in absolute value). In the present thesis, the excretion parameters for flavonoid conjugates excreted via bile, urine, and intestinal efflux back to the intestinal lumen were derived by fitting the predicted time-dependent plasma concentrations of total flavonoid (aglycone and metabolites) with experimental human data. The disadvantage of this method is that it can be only applied when there are in vivo data available as the fitted parameters are dependent on the in vivo data used for the fitting. For other flavonoids, where there is a lack of in vivo data, this method is not applicable. Therefore, to define PBK models for other flavonoids and to gain insight in the importance of the major excretion route and forms for the ADME of flavonoids, a method that can predict excretion parameters without the need for in vivo data would be valuable. Available alternative methods to derive the kinetic parameters for biliary and urinary excretion, and for intestinal efflux back to the intestinal lumen are as follow: 
1. Transfected cells with transporter proteins of interest provide the most simple model and have been extensively used to characterize polymorphisms, substrate specificity, and structure-activity-relationships for efflux transporter proteins [21-27]. Transfected cells with transporter proteins of interest can be prepared from baculovirus infected Sf9 cells as well as from mammalian cells (e.g. LLC-PK1, MDCKII, HeLa, HEK293T) [21-28]. Excretion of flavonoid metabolites via bile, urine, and intestinal efflux back to the intestinal lumen in humans, have been reported to be mediated by ATP binding cassette (ABC) transporters [29]. ABC transporters use energy from ATP hydrolysis as their driving force and are found on extracellular and intracellular membranes of tissue barriers and excretory organs [29]. Pglycoprotein, breast cancer resistant protein, and multidrug resistant-associated protein 2 have been reported to be the major $\mathrm{ABC}$ transporters mediating the excretion of flavonoid metabolites via bile, urine, and intestinal efflux back to the intestinal lumen in humans $[26,28$, 30-37]. When using the transfected cells with transporter proteins of interest, it is of importance to know the differences in expression levels of active transporters between in vitro models and human organs to determine the scaling factors to translate the in vitro determined values to in vivo relevant input parameters for PBK models. The expression levels of active transporters in human intestine, liver, and kidney are available in literature [38].

2. Immobilized human cell line monolayers cultured on a permeable membrane in a two-compartment cell culture system (apical and basolateral compartment) have been used in a number of studies [31, 39, 40]. A variety of cells can be used for these transporter assays, in which the cells must express the efflux transporters of interest and form a functionally polarized cell monolayer, complete with tight extracellular junctions [40]. Several human derived cell lines have met these requirements including the human liver-derived cell lines Fa2N-4, HepG2, Hep3B, PLc/PRFs Huh7, HBG, and HepaRG [41], the human renal cell lines HK-2, Caki-1, RPTEC/TERT1 and ciPTEC [39, 42, 43], and the human intestinal Caco-2 cell lines [31, 44] available for studying excretion via bile, urine, and intestinal efflux back to the intestinal lumen, respectively. The kinetic parameters for excretion derived from the immobilized human cell line monolayer need to be corrected for the differences in the expression of active transporters between human cell lines and human tissues to determine the scaling factors for translation of in vitro excretion parameters to in vivo relevant input parameters for the PBK models. The differences in expression of active transporters between human cell lines and human organs are available in literature $[38,45,46]$.

3. Primary human cells are ideal for performing transporter assays, as primary cells are isolated from human organs and thus may restrain and display important in vivo anatomical and 


\section{Chapter 6}

functional characteristics $[47,48]$. Furthermore, each culture is derived from cells isolated from a single donor enabling a study on interindividual human variation in transporters in the respective organ. So far, primary human cells that are available include human hepatocytes and human intestinal epithelial cells [47, 48]. Even though primary human cells are useful tools for studying uptake and efflux characteristics of substances as well as the consequences of interindividual human variation in active transporters, primary human cells are difficult to culture, not readily available and expensive which seems to have so far hampered their use in defining rate constants for biliary excretion and intestinal efflux back to the intestinal lumen in PBK modeling.

Development of QSAR models to derive kinetic constants for formation of different flavonoid conjugates

The development of PBK models as described in the present thesis is undeniable a laborious and time-consuming process as a large number of model parameters are required and need to be defined. In the studies described in the present thesis, a lot of time was dedicated to the performance of in vitro incubations with human tissue fractions to derive kinetic parameters for formation of the different flavonoid conjugates. For the future use of PBK models in exploring the ADME characteristics for a wider range of flavonoids, there is a need to shorten the time needed to derive the kinetic parameters for metabolic conversions. Alternative methods to derive such parameters could be based on the development of in silico based quantitative structure activity relationships (QSARs). QSAR models should be developed for glucuronidation, sulfation, and methylation of flavonoids as these three pathways are important for the formation of the major circulating flavonoid metabolites. Knowledge on structure activity relationships on the regioselectivity of different UGT and SULT isoforms on formation of different monoglucuronides and monosulfates are available in literature [49-51]. This knowledge will be useful for the development of the QSAR models to determine kinetic constants for formation of different flavonoid conjugates. The standard approach for development of QSAR models is to relate molecular descriptors of chemicals to specific endpoints of interest. General guidelines for QSAR development as well as their validation are available in literature [52]. Even though the development of QSAR models is laborious and time-consuming as well, the QSAR models developed will be effective tools to predict kinetic parameters for conversion of large numbers of flavonoids into their respective conjugates and ultimately accelerate the development of PBK models for other flavonoids. 
So far, there are QSAR models available for some human UGT [53-55], SULT [56], and COMT [57, 58] isoforms. For glucuronidation reactions, the first generalized QSAR models were developed for known human UGT1A1 substrates including flavonoids to predict substrate selectivity and binding affinity of inhibitors (i.e. apparent inhibition constant, $K_{\mathrm{i}, \text { app }}$ ), revealing the importance of two hydrophobic domains separated from the glucuronidation site by $4 \AA$ and $7 \AA$, respectively, for the prediction of $K_{\mathrm{i} \text {,app }}$ [53]. Ethell et al. [54] developed the QSAR models to predict the kinetic parameters (i.e. the maximum velocity $\left(V_{\max }\right)$ and Michaelis-Menten constant $\left.\left(K_{\mathrm{m}}\right)\right)$ for glucuronidation of 4-substituted phenols by human UGT1A6 and UGT1A9. The results suggested that the size of the 4-substituent and the bulkiness of substrate are important factors because when the size of the 4-substituent decreased, the $V_{\max }$ and $K_{\mathrm{m}}$ values increased and decreased, respectively, and bulky substrates might be accepted into the binding site but might not be glucuronidated, or only weakly glucuronidated [54]. Recently, Wu et al. [55] successfully developed QSAR models for predicting the kinetic parameters (i.e. $V_{\max }$ and intrinsic clearance $\left(\mathrm{CL}_{\mathrm{in}}=V_{\max } / K_{\mathrm{m}}\right)$ ) for 3-Oglucuronidation of flavonoids by human UGT1A9. The results obtained from this study revealed the key structural features including that the presence of multiple electropositive atoms or groups (e.g. - $\mathrm{OH})$ to the B-ring would result in poor 3-O-glucuronidation by human UGT1A9, contradictory the presence of a bulky substituent at C6 or electronegative atoms or groups (e.g. - $\mathrm{Cl},-\mathrm{F},-\mathrm{OCH}_{3}$ ) around the B-ring would result in fast glucuronidation of the 3-OH [55]. Additionally, this study highlighted the important role of electrostatic potential (i.e. electronegative versus electropositive entity) in determining human UGT1A9-mediated 3-OH glucuronidation of flavonoids [55]. For sulfation reactions, there is a QSAR model available for predicting $K_{\mathrm{m}}$ values for formation of phenol sulfation by human SULT1A3 [56]. This study indicated that phenols with bulky substituents in the para-position have high $K_{\mathrm{m}}$ values compared to ortho- and meta-substituted analogues and an electronic interaction near Glu146 in the human SULT1A3 structure was an important structural feature for determining human SULT1A3-mediated phenol sulfation [56]. For methylation reactions, there are two QSAR models available for predicting kinetic parameters (i.e. $K_{\mathrm{m}}, V_{\max }$, and $\mathrm{CL}_{\mathrm{in}}$ ) for methylation of catecholic substrates by human soluble, cytosolic COMT (S-COMT) [57, 58]. The results obtained from these studies suggested the importance of substrate bulkiness and electronic parameters [57, 58]. Altogether, the QSAR models available can be used as a platform for the development of QSAR models for other UGT, SULT and COMT isoforms and other conjugation positions focusing on the major isoforms involved in metabolism of flavonoids. Ultimately, combing the predictions from all QSAR models for individual isoform, will allow 


\section{Chapter 6}

the estimation of the kinetic parameters for overall glucuronidation, sulfation, and methylation of different flavonoid conjugates. Obviously, it remains to be established whether this approach would be more time effective than measurements of the respective kinetic parameters using relevant in vitro models

\section{Quality of in vivo data on metabolic patterns in plasma for validation of the PBK models}

Once a PBK model is developed, it is crucial to evaluate the performance of the newly defined models by comparing the prediction with experimental in vivo data, supporting validity of the model and as a consequence supporting the model predictions. The quality of in vivo data used to evaluate the performance of the model is of importance. In the present thesis, the PBK models were developed to predict dose-dependent plasma concentrations of flavonoid aglycones and their metabolites as well as to tentatively identify the type and conjugation position of the circulating metabolites. To facilitate the model validation, there is a need for in vivo data on metabolic plasma profiles of flavonoids that identify and quantify individual circulating metabolites. Even though flavonoids have been intensively studied for decades [59], surprisingly there are only limited number of in vivo studies available that provide these data [12]. In most studies, flavonoid conjugates in plasma were identified and quantified from the amounts of aglycone liberated from conjugated metabolites by using $\beta$-glucuronidase and sulfatase to deconjugate the conjugated metabolites to their respective aglycone [60-68]. This method has been criticized for running a risk of underestimation and lack of information on the absolute structural conformation of flavonoid conjugates especially with respect to the conjugation position $[60,61]$. The use of advanced analytical methods in chromatography and mass spectrometry can also not provide the absolute conformation of the chemical structure of individual flavonoid conjugates [69]. Nuclear magnetic resonance (NMR) spectroscopy is a powerful tool to identify unknown chemicals [70] and in this case can be used to identify the conjugation position of individual flavonoid conjugates. However, ${ }^{1} \mathrm{H}-\mathrm{NMR}$ measurements require relatively large amounts of the tested compounds [70], which might hamper the use of NMR to identify conjugation position of individual flavonoid conjugates. Alternative methods would be the use of in silico approaches to predict the ${ }^{1} \mathrm{H}-\mathrm{NMR}$ spectra of possible flavonoid conjugates together with the Metabolite Identification Database (MetIDB), a publicly accessible database of predicted and experimental ${ }^{1} \mathrm{H}-\mathrm{NMR}$ spectra of flavonoids, to identify flavonoid conjugates [70, 71]. Without NMR data unambiguous identification of flavonoid metabolites requires available standard compounds for the respective flavonoid conjugates [7276]. However, there is a general lack of commercially available standard compounds for 
flavonoid conjugates and the commercially available standard compounds are generally expensive. Standard compounds for flavonoid conjugates can be chemically synthesized, although methods for chemical synthesis are only available for a limited number of flavonoid conjugates and are quite complex, expensive, laborious, and require specific knowledge and equipment [76-78]. Alternative methods would be the use of biosynthesis together with ${ }^{1} \mathrm{H}$ NMR using MetIDB to identify the structure of biosynthesized flavonoid conjugates as recently described by Beekmann et al. [71]. Altogether, future in vivo studies on flavonoid metabolism will need to identify and quantify individual circulating metabolites of flavonoids as this information is of importance for validation of the PBK model performance as well as to gain more insight in in vivo effects of flavonoids.

\section{Deconjugation of flavonoid conjugates at the site of action}

Flavonoids are mainly present as conjugates, which represent most likely the forms that reach the target tissues. These conjugates might be deconjugated to their respective aglycones at the target tissue by $\beta$-glucuronidases, deconjugating enzymes. Lysosomal $\beta$-glucuronidase is reported to be present in high concentrations in neutrophils and eosinophils [79]. Upon stimulation, these inflammatory cells release $\beta$-glucuronidase into the extracellular space, leading to an increase in local $\beta$-glucuronidase activity and blood $\beta$-glucuronidase concentrations during inflammation [79]. In vitro studies with flavonoid monoglucuronides reported an increase in the respective aglycones in human neutrophils stimulated with LPS [80, 81]. Pre-clinical cancer studies with glucuronidated drugs also found an increase in unconjugated drug concentrations in necrotic areas of human pancreatic and lung carcinomas $[82,83]$. Besides $\beta$-glucuronidase activity at the site of inflammation, human breast tissue has also been reported to contain $\beta$-glucuronidase as Islam et al. demonstrated that genistein-7-Oglucuronide was deconjugated to genistein aglycone by human breast $S 9$ fractions [14]. The PBK models defined in the present thesis do not yet include deconjugation of flavonoid glucuronides at the site of action, because of the lack of human data to perform and evaluate this simulation. For further refinement of the predictions by the PBK model, insight in deconjugation of flavonoid glucuronides at the site of action should be studied to a further extent including determining kinetic constants for deconjugation of flavonoid glucuronides at the site of action. 
Chapter 6

\section{Matrix effects on the ADME of flavonoids}

It is of importance to note that flavonoids are normally present in a complex food matrix (containing additional ingredients such as proteins, carbohydrates, fiber, fat, alcohol), which might influence the ADME of flavonoids in vivo. Dissolving cocoa powder in whole milk has been reported to alter the metabolic profiles of (-)-epicatechin excreted in urine of healthy human volunteers as there was an increase in the amount of sulfated conjugates accompanied by a decrease in glucuronidated conjugates as compared with what was formed when cocoa powder was administered dissolved in water [84]. Carbohydrate composition of the food matrix has been suggested to be a key factor in determining the cocoa flavanols absorption and metabolism in the small intestine as maltitol-containing chocolate decreased in levels of total flavanol absorption and peak plasma concentrations of unmethylated epicatechin as compared with the sucrose-containing equivalent [85]. The sugar content of the matrix has been reported to lower bioavailability of resveratrol in grape juice in comparison with the pure aglycone [86]. Consumption of quercetin with a fat-rich diet has been reported to increase the plasma AUC of total quercetin (aglycone and metabolites) by approximately $45 \%$ as compared with subjects who consumed quercetin with a fat-free diet [87]. The matrix effect of dietary fibers has been in general found to decrease the absorption of flavonoids [88]. Besides matrix effects of food components, the presence of other dietary flavonoids and other phytochemicals has also been also suggested to influence the ADME of flavonoids. Brand et al. [89] demonstrated that the amount of hesperetin metabolites excreted to the basolateral side of a Caco- 2 cell monolayer increased when hesperetin was co-administered together with other flavonoids. This implies that when hesperetin is consumed together with other flavonoids such as quercetin, the plasma concentrations of hesperetin metabolites may be increased. Overall, the food matrix can influence the ADME characteristics of flavonoids, which can therefore alter the plasma pharmacokinetics of flavonoids in vivo. For the future refinement of the PBK models, food matrix effects should be considered for a more realistic description of plasma metabolic patterns upon flavonoid consumption in vivo.

\section{Microbial metabolism of flavonoids}

Aside from the availability of flavonoid conjugates in the systemic circulation, several small phenolic molecules have also been detected as metabolites in human plasma and urine in some human intervention studies with flavonoids [90]. These small phenolic molecules are likely the degradation products of flavonoid aglycones and their metabolites formed by gut microbiota [90]. There is a lack of human data on plasma concentrations of microbial 
metabolites of flavonoids, since most of the studies focused on the conjugated flavonoids. Furthermore, the contributions of these microbial metabolites to biological effects of flavonoids in humans in vitro and in vivo are not well-characterized [90]. An exception is made for equol, a well-known estrogenic microbial metabolite of daidzein [91, 92]. Therefore, future development of the PBK models defined in the present thesis should include the microbial metabolism of flavonoids. Also, more research should be performed to gain insight in the role of microbial metabolites of flavonoids in biological effects of flavonoids in human in vivo.

\subsubsection{In vitro biological studies of flavonoid mediated effects}

In vitro studies are important tools for better understanding the modes of action of flavonoids that exert specific health effects. In the past in vitro biological studies of flavonoids were often performed using flavonoid aglycones, which are, as also shown by the results of the present thesis, hardly bioavailable in vivo [59]. Given that flavonoids are present in the systemic circulation as their conjugates, it is now undoubtedly clear that in vitro studies on biological activities of flavonoids should also focus on flavonoid conjugates, reflecting physiological relevant forms of flavonoids. The PBK models developed can be used as guidelines for the experimental design of such in vitro studies by enabling definition of the major available forms of a flavonoid in the systemic circulation, providing information on the type and conjugation position and thus the type of conjugates to be preferably tested in vitro. Based on the PBK model predictions in the present thesis, monoglucuronides can be expected to represent the major circulating metabolites in humans, followed by mixed conjugates containing a glucuronic acid and/or a sulfate moiety and monosulfates. The recent review by Beekmann et al. [10] revealed that dependent on the type and conjugation position and in vitro endpoint studied, some conjugates could restrain some activity and in some cases conjugates exerted even higher activity than their respective aglycone. These results indicate that in vivo biological activities of flavonoids could be partly ascribed to their metabolites. The biological activity of hesperetin and its major circulating metabolites (i.e. hesperetin-3'-O-glucuronide and hesperetin-7-Oglucuronide) on PKA activity obtained from in vitro bioassay in the present thesis (Chapter 5), showed that the two metabolites were at least equally active in inhibition of PKA activity as hesperetin aglycone. Kay [93] reported that at concentrations identified in plasma in human studies, flavonoid aglycones are likely to have only limited activity, whereas their metabolites (methyl, glucuronide, sulfate conjugates) were active across varies bioassays. Furthermore, when these metabolites were applied together as mixtures, some were often just as active and, in a few cases, even more active than when applied individually [93]. Therefore, it is also of 


\section{Chapter 6}

importance to investigate in vitro biological activity of mixtures of the major circulating metabolites of flavonoids. And to the extent possible, in vitro concentration-response curves for specific biological activities of flavonoid aglycone but also of its major circulating metabolites should be derived.

Apart from conjugated metabolites, it is of importance to determine the contribution of microbial metabolites of flavonoids to in vivo biological activities of flavonoids, as microbial metabolites will also be available in the systemic circulation (see section 6.2.1 on Microbial metabolism of flavonoids). Aside from equol a potent estrogenic microbial metabolite of daidzein [91, 92], biological activities of other microbial flavonoid metabolites are not well established in vitro [90]. Based on the review article by Aura [90], some microbial metabolites of flavonoids were found to exert biological activities in in vitro bioassays on estrogenicity, inhibition of hepatocellular cholesterol synthesis, and inflammatory activity. Another aspect that should be implemented in in vitro methods, is to setup a standard methodology for in vitro measurements on each specific endpoint that could help to minimize the inter-laboratory differences in the obtained results. For example, in vitro data available for genistein induced ER $\alpha$ mediated cell proliferation (Chapter 4 of the thesis) show large variation, which is likely due to differences between the laboratories where the cells were cultivated, as highlighted by Osborne et al. [94] who reported that even though MFC-7 cell lines obtained from different laboratories have similar karyotype, they may have unique biological properties. And before concluding on any in vivo biological effects, PBK model based reverse dosimetry as performed in Chapter 4 and 5 of the present thesis seems to be an essential step to be included to enable analysis of whether in vitro effects can be expected at realistic human dose levels.

\subsubsection{Use of PBK model based reverse dosimetry}

The present thesis demonstrated the use of PBK model based reverse dosimetry to predict in vivo effective dose levels of flavonoids (Chapter 4 and 5). The in vitro-in silico approach applied in the present thesis provides knowledge on pharmacokinetics and pharmacodynamics of flavonoids and would be useful to establish dose levels of flavonoids at which certain in vitro biological endpoint can be achieved in humans in vivo. The following sections discuss how to translate in vitro data to in vivo dose levels and what are the levels of uncertainty in the predicted in vivo $\mathrm{BMD}_{05}$ (benchmark dose for $5 \%$ response) values predicted based on in vitro endpoints by using PBK model based reverse dosimetry. 
Translation of in vitro data to in vivo dose levels

When performing a PBK-model based reverse dosimetry study, it is important to first select the in vitro endpoint that is relevant to the in vivo effects, and that can be derived from an available in vitro test system that mimics the in vivo situation as closely as possible, and thus allows a construction of a suitable in vitro concentration-response curve. Such an in vitro concentration-response curve can be translated to an in vivo dose-response curve by relating the in vitro concentration to the maximum plasma concentration $\left(\mathrm{C}_{\max }\right)$ as in the present thesis (Chapter 4 and 5) or to the area under the plasma concentration-time curve (AUC) of the flavonoid aglycone or of a relevant mixture of the aglycone and its metabolites. The choice of the dose metric (plasma $\mathrm{C}_{\max }$ or plasma AUC) is dependent on the time-course of the effects; a short term and/or reversible effect is more likely to be related to the plasma $\mathrm{C}_{\max }$ and vice versa long term and/or irreversible effects might likely be better related to the plasma AUC. When relating the in vitro endpoint to the plasma AUC, the in vitro AUC values of the tested chemical should be estimated by measuring the change in concentration of the tested chemical in the culture medium at multiple time points over the assay time [95] or if the concentration of the tested chemical remains constant in the culture medium over the assay time the applied in vitro concentration can be multiplied with assay time to estimate the in vitro AUC value [96]. Prior to translation of the in vitro concentration-response curve to the in vivo dose-response curve, it is essential to consider that only the free fraction of the chemical will likely exert the effects in vitro and in vivo, implying a requirement for a correction for the binding of the chemical to protein and lipids under the in vitro and the in vivo conditions $[97,98]$. The free fraction of the chemical can be measured using in vitro methods available in literature [96] or in silico methods as illustrated in the present thesis (Chapter 4 and 5) [98, 99]. By correcting for the differences in protein and lipid concentrations between in vitro and in vivo, each nominal in vitro concentration can be extrapolated to an in vivo effect concentration. The in vivo effect concentration (or AUC) obtained is set equal to the plasma $\mathrm{C}_{\max }$ (or plasma $\mathrm{AUC}$ ) of the flavonoid aglycone or mixtures of the aglycone and its metabolites. The PBK model is subsequently used to calculate the corresponding oral dose levels in humans, providing the in vivo dose-response curve. The in vivo dose-response curve obtained can be used to subsequently derive the in vivo $\mathrm{BMD}_{05}$ values for the endpoint of interest. 
Chapter 6

Uncertainty factors for the predicted in vivo $B M D_{05}$ values

In the present thesis, a PBK model based reverse dosimetry approach was applied to predict in vivo $\mathrm{BMD}_{05}$ values based on in vitro data to establish dose levels of flavonoids at which certain in vitro biological endpoint can be achieved in humans in vivo. There are a number of uncertainties that can affect the predicted in vivo $\mathrm{BMD}_{05}$ values and need to be considered for a better interpretation of the results and to further increase the accuracy of the predictions in the future. The kinetic parameters for metabolic conversions of flavonoids are influential parameters that affect the plasma concentrations of flavonoid aglycones and their metabolites based on the sensitivity analysis obtained in the present thesis. This indicates that individual human variation in conjugating enzymes will influence the predicted plasma concentrations of flavonoid aglycones and their metabolites and thus affect the predicted in vivo $\mathrm{BMD}_{05}$ values. Besides interindividual human variation in conjugating enzymes, other factors such as genetic and lifestyle factors influencing the enzymatic expression and/or activity of the conjugating enzymes [100], variation in dietary intake of flavonoids, and matrix effects can also influence the predicted in vivo $\mathrm{BMD}_{05}$ values. The predicted in vivo $\mathrm{BMD}_{05}$ values for biological effects of flavonoids in humans in the present thesis (Chapter 4 and 5) do not yet take these uncertainties into consideration. These uncertainties due to interindividual human variations might explain the difference in the predicted metabolic plasma profiles and the predicted in vivo $\mathrm{BMD}_{05}$ values with reported human data. Altogether, for a better accuracy on the predicted metabolic plasma profiles and in vivo dose levels for biological effects of flavonoids in humans in vivo, all above mention uncertainties need to be carefully considered and better defined to determine how much these uncertainties related to interindividual human variation contribute to variation in the biological effects of flavonoids in the population.

\subsection{Conclusions}

Altogether, the present thesis shows that PBK modeling is a powerful tool to gain insight not only in dose-dependent plasma concentrations of flavonoid aglycones and their metabolites, but also to tentatively identify the type and conjugation position of the circulating metabolites. These insights are of importance to better understand in vivo effects of flavonoids because conjugations determine the form of the flavonoids available in the systemic circulation in vivo. Thus, in this respect PBK model based predictions of the type and conjugation position and the concentration of possible metabolites is expected to provide better insight in key parameter determining the in vivo effects of flavonoids [10]. The present thesis also demonstrated the use of PBK models to assess inter- and intraspecies differences in ADME of flavonoids taking 
quercetin as the model compound. Furthermore, the PBK models developed were demonstrated to be of use as a platform for the development of PBK models for other flavonoids and to guide experimental design of in vitro studies. Last but not least, the PBK models developed were shown to facilitate the translation of in vitro concentration-response curves to in vivo doseresponse curves to determine whether and if so at what oral dose levels flavonoids may elicit biological activity in vivo, providing an in vitro-in silico approach and eliminating the need of human intervention studies. It is concluded that PBK modeling provides a useful additional research tool for studies on pharmacokinetics and pharmacodynamics of flavonoids presenting opportunities that are not easily provided by other methods. 


\section{References}

1. Erlund, I., Review of the flavonoids quercetin, hesperetin, and naringenin. Dietary sources, bioactivities, bioavailability, and epidemiology. Nutrition Research, 2004. 24(10): p. 851-874.

2. Neuhouser, M. L., Review: dietary flavonoids and cancer risk: evidence from human population studies. Nutrition and Cancer, 2004. 50(1): p. 1-7.

3. Perez-Vizcaino, F. and J. Duarte, Flavonols and cardiovascular disease. Molecular Aspects of Medicine, 2010. 31(6): p. 478-494.

4. Hughes, L. A., et al., Higher dietary flavone, flavonol, and catechin intakes are associated with less of an increase in BMI over time in women: a longitudinal analysis from the Netherlands Cohort Study. The American Journal of Clinical Nutrition, 2008. 88(5): p. 1341-1352.

5. Liu, Y.-J., et al., Dietary flavonoids intake and risk of type 2 diabetes: A meta-analysis of prospective cohort studies. Clinical Nutrition, 2014. 33(1): p. 59-63.

6. Letenneur, L., et al., Flavonoid intake and cognitive decline over a 10-year period. American Journal of Epidemiology, 2007. 165(12): p. 1364-1371.

7. Morris, M. C., et al., Associations of vegetable and fruit consumption with age-related cognitive change. Neurology, 2006. 67(8): p. 1370-1376.

8. Havsteen, B. H., The biochemistry and medical significance of the flavonoids. Pharmacology \& Therapeutics, 2002. 96(2-3): p. 67-202.

9. Williamson, G., et al., In vitro biological properties of flavonoid conjugates found in vivo. Free Radical Research, 2005. 39(5): p. 457-469.

10. Beekmann, K., et al., A state-of-the-art overview of the effect of metabolic conjugation on the biological activity of flavonoids. Food \& Function, 2012. 3(10): p. 1008-1018.

11. Chen, Z., et al., Metabolism of flavonoids in human: a comprehensive review. Current Drug Metabolism, 2014. 15(1): p. 48-61.

12. Manach, C., et al., Bioavailability and bioefficacy of polyphenols in humans. I. Review of 97 bioavailability studies. The American Journal of Clinical Nutrition, 2005. 81(1): p. 230S-242S.

13. Yuan, B., et al., Role of metabolism in the effects of genistein and its phase II conjugates on the growth of human breast cell lines. The AAPS Journal, 2012. 14(2): p. 329-344.

14. Islam, M. A., et al., Deconjugation of soy isoflavone glucuronides needed for estrogenic activity. Toxicology in Vitro, 2015. 29(4): p. 706-715.

15. Rietjens, I. M. C. M., et al., Mechanisms underlying the dualistic mode of action of major soy isoflavones in relation to cell proliferation and cancer risks. Molecular Nutrition \& Food Research, 2013. 57(1): p. 100-113.

16. EFSA, Risk assessment for peri- and post-menopausal women taking food supplements containing isolated isoflavones. EFSA Journal 2015, 2015. 13(10): p. 4246 [page 342].

17. Morand, C., et al., Hesperidin contributes to the vascular protective effects of orange juice: a randomized crossover study in healthy volunteers. Am J ClinNutr, 2011. 93.

18. Milenkovic, D., et al., Hesperidin displays relevant role in the nutrigenomic effect of orange juice on blood leukocytes in human volunteers: a randomized controlled cross-over study. PLoS ONE, 2011. 6(11): p. e26669.

19. Sánchez-Moreno, C., et al., High-pressurized orange juice consumption affects plasma vitamin $C$, antioxidative status and inflammatory markers in healthy humans. The Journal of Nutrition, 2003. 133(7): p. 2204-2209.

20. Punt, A., et al., Use of physiologically based biokinetic (PBBK) modeling to study estragole bioactivation and detoxification in humans as compared with male rats. Toxicological Sciences, 2009. 110(2): p. 255-269. 
21. van Zanden, J. J., et al., The effect of quercetin phase II metabolism on its MRP1 and MRP2 inhibiting potential. Biochemical Pharmacology, 2007. 74(2): p. 345-351.

22. Glavinas, H., et al., Utilization of membrane vesicle preparations to study drug-ABC transporter interactions. Expert Opinion on Drug Metabolism \& Toxicology, 2008. 4(6): p. 721-732.

23. $\mathrm{Wu}, \mathrm{C} .-\mathrm{P}$. , et al., Modulatory effects of plant polyphenols on human multidrug resistance proteins 1, 4, and 5 (ABCC1, 4, and 5). The FEBS journal, 2005. 272(18): p. 4725-4740.

24. Kondo, C., et al., Functional analysis of SNPs variants of BCRP/ABCG2. Pharmaceutical Research, 2004. 21(10): p. 1895-1903.

25. Wei, Y., et al., Revolving door action of BCRP facilitates or controls the efflux of favone glucuronides from UGT1A9-overexpressing HeLa Cells. Molecular pharmaceutics, 2013. 10(5): p. 1736-1750.

26. van Zanden, J. J., et al., Quantitative structure activity relationship studies on the flavonoid mediated inhibition of multidrug resistance proteins 1 and 2. Biochemical Pharmacology, 2005. 69(4): p. 699-708.

27. Létourneau, I. J., R. G. Deeley, and S. P. C. Cole, Functional characterization of nonsynonymous single nucleotide polymorphisms in the gene encoding human multidrug resistance protein 1 (MRP1/ABCC1). Pharmacogenetics and Genomics, 2005. 15(9).

28. Imai, Y., et al., Phytoestrogens/flavonoids reverse breast cancer resistance protein/ABCG2mediated multidrug resistance. Cancer Research, 2004. 64(12): p. 4346.

29. Sabina, P., et al., Bioavailability of flavonoids: a review of their Membrane transport and the function of bilitranslocase in animal and plant organisms. Current Drug Metabolism, 2009. 10(4): p. 369-394.

30. Wang, Y., J. Cao, and S. Zeng, Involvement of P-glycoprotein in regulating cellular levels of Ginkgo flavonols: quercetin, kaempferol, and isorhamnetin. Journal of Pharmacy and Pharmacology, 2005. 57(6): p. 751-758.

31. Brand, W., et al., Metabolism and transport of the citrus flavonoid hesperetin in caco-2 cell monolayers. Drug Metabolism and Disposition, 2008. 36(9): p. 1794-1802.

32. Cao, J., et al., Role of P-glycoprotein in the intestinal absorption of glabridin, an active flavonoid from the root of Glycyrrhiza glabra. Drug Metabolism and Disposition, 2007. 35(4): p. 539-553.

33. Zhang, L., et al., Hepatic metabolism and disposition of baicalein via the coupling of conjugation enzymes and transporters - in vitro and in vivo evidences. The AAPS Journal, 2011. 13(3): p. 378-389.

34. Xu, H., et al., Disposition of naringenin via glucuronidation pathway Is affected by compensating Eeflux transporters of hydrophilic glucuronides. Molecular Pharmaceutics, 2009. 6(6): p. 1703-1715.

35. Williamson, G., et al., Interaction of positional isomers of quercetin glucuronides with the transporter ABCC2 (cMOAT, MRP2). Drug Metabolism and Disposition, 2007. 35(8): p. 12621268.

36. O'Leary, K. A., et al., Metabolism of quercetin-7- and quercetin-3-glucuronides by an in vitro hepatic model: the role of human B-glucuronidase, sulfotransferase, catechol-Omethyltransferase and multi-resistant protein 2 (MRP2) in flavonoid metabolism. Biochemical Pharmacology, 2003. 65(3): p. 479-491.

37. Romiti, N., et al., Effects of grapefruit juice on the multidrug transporter P-glycoprotein in the human proximal tubular cell line HK-2. Life Sciences, 2004. 76(3): p. 293-302. 
38. Hilgendorf, C., et al., Expression of thirty-six drug transporter genes in human intestine, liver, kidney, and organotypic cell lines. Drug Metabolism and Disposition, 2007. 35(8): p. 13331340 .

39. Jenkinson, S. E., et al., The limitations of renal epithelial cell line HK-2 as a model of drug transporter expression and function in the proximal tubule. Pflügers Archiv - European Journal of Physiology, 2012. 464(6): p. 601-611.

40. Zhang, Y., C. Bachmeier, and D. W. Miller, In vitro and in vivo models for assessing drug efflux transporter activity. Advanced Drug Delivery Reviews, 2003. 55(1): p. 31-51.

41. Soldatow, V. Y., et al., In vitro models for liver toxicity testing. Toxicology research, 2013. 2(1): p. 23-39.

42. Wilmer, M. J., et al., Novel conditionally immortalized human proximal tubule cell line expressing functional influx and efflux transporters. Cell and Tissue Research, 2010. 339(2): p. 449-457.

43. Wieser, M., et al., hTERT alone immortalizes epithelial cells of renal proximal tubules without changing their functional characteristics. American Journal of Physiology - Renal Physiology, 2008. 295(5): p. F1365.

44. Chabane, M. N., et al., Quercetin and naringenin transport across human intestinal Caco-2 cells. Journal of Pharmacy and Pharmacology, 2009. 61(11): p. 1473-1483.

45. Seithel, A., et al., Variability in mRNA expression of ABC-and SLC-transporters in human intestinal cells: Comparison between human segments and Caco-2 cells. European Journal of Pharmaceutical Sciences, 2006. 28(4): p. 291-299.

46. Englund, G., et al., Regional levels of drug transporters along the human intestinal tract: Coexpression of ABC and SLC transporters and comparison with Caco-2 cells. European Journal of Pharmaceutical Sciences, 2006. 29(3-4): p. 269-277.

47. Hamilton, G. A., et al., Regulation of cell morphology and cytochrome P450 expression in human hepatocytes by extracellular matrix and cell-cell interactions. Cell and Tissue Research, 2001. 306(1): p. 85-99.

48. Kauffman, A. L., et al., Alternative functional in vitro models of human intestinal epithelia. Frontiers in Pharmacology, 2013. 4: p. 79.

49. Wu, B., et al., First-pass metabolism via UDP-glucuronosyltransferase: a barrier to oral bioavailability of phenolics. Journal of Pharmaceutical Sciences, 2011. 100(9): p. 3655-3681.

50. Wong, Y. C., et al., Structure-activity relationships of the glucuronidation of flavonoids by human glucuronosyltransferases. Expert Opinion on Drug Metabolism \& Toxicology, 2009. 5(11): p. 1399-1419.

51. $\mathrm{Wu}, \mathrm{B} .$, et al., Regioselective sulfation and glucuronidation of phenolics: insights into the structural basis of conjugation. Current drug metabolism, 2011. 12(9): p. 900-916.

52. Cherkasov, A., et al., QSAR modeling: where have you been? where are you going to? Journal of Medicinal Chemistry, 2014. 57(12): p. 4977-5010.

53. Sorich, M. J., et al., Pharmacophore and quantitative structure activity relationship modelling of UDP-glucuronosyltransferase 1A1 (UGT1A1) substrates. Pharmacogenetics and Genomics, 2002. 12(8): p. 635-645.

54. Ethell, B. T., et al., Quantitative structure activity relationships for the glucuronidation of simple phenols by expressed human UGT1A6 and UGT1A9. Drug Metabolism and Disposition, 2002. 30(6): p. 734.

55. $\mathrm{Wu}, \mathrm{B}$. , et al., Three-dimensional quantitative structure-activity relationship studies on UGT1A9-mediated 3-O-glucuronidation of natural Ffavonols using a pharmacophore-based comparative molecular field analysis model. The Journal of Pharmacology and Experimental Therapeutics, 2011. 336(2): p. 403-413. 
56. Sipilä, J., et al., CoMFA modeling of enzyme kinetics: Km values for sulfation of diverse phenolic substrates by human catecholamine sulfotransferase SULT1A3. Journal of Chemical Information and Computer Sciences, 2003. 43(5): p. 1563-1569.

57. Sipilä, J. and J. Taskinen, CoMFA modeling of human catechol O-methyltransferase enzyme kinetics. Journal of Chemical Information and Computer Sciences, 2004. 44(1): p. 97-104.

58. Costescu, A., et al., QSAR modeling of human catechol O-methyltransferase enzyme kinetics. Journal of Mathematical Chemistry, 2009. 45(2): p. 287-294.

59. Kay, C. D., The future of flavonoid research. British Journal of Nutrition, 2010. 104(SupplementS3): p. S91-S95.

60. Crozier, A., I. B. Jaganath, and M. N. Clifford, Dietary phenolics: chemistry, bioavailability and effects on health. Natural Product Reports, 2009. 26(8): p. 1001-1043.

61. Gu, L., et al., Isoflavone conjugates are underestimated in tissues using enzymatic hydrolysis. Journal of Agricultural and Food Chemistry, 2005. 53(17): p. 6858-6863.

62. Erlund, I., et al., Plasma kinetics and urinary excretion of the flavanones naringenin and hesperetin in humans after ingestion of orange juice and grapefruit juice. The Journal of Nutrition, 2001. 131(2): p. 235-241.

63. Manach, C., et al., Bioavailability in humans of the flavanones hesperidin and narirutin after the ingestion of two doses of orange juice. 2003. 57(2): p. 235-242.

64. Nielsen, I. L. F., et al., Bioavailability is improved by enzymatic modification of the citrus flavonoid hesperidin in humans: a randomized, double-blind, crossover trial. The Journal of Nutrition, 2006. 136(2): p. 404-408.

65. Gardana, C., et al., Flavanone plasma pharmacokinetics from blood orange juice in human subjects. British Journal of Nutrition, 2007. 98(01): p. 165-172.

66. Mullen, W., et al., Bioavailability and metabolism of orange juice flavanones in humans: impact of a full-fat yogurt. Journal of Agricultural and Food Chemistry, 2008. 56(23): p. 11157-11164.

67. Brett, G. M., et al., Absorption, metabolism and excretion of flavanones from single portions of orange fruit and juice and effects of anthropometric variables and contraceptive pill use on flavanone excretion. British Journal of Nutrition, 2009. 101(05): p. 664-675.

68. Egert, S., et al., Daily quercetin supplementation dose-dependently increases plasma quercetin concentrations in healthy Humans. The Journal of Nutrition, 2008. 138(9): p. 1615-1621.

69. March, R. and J. Brodbelt, Analysis of flavonoids: Tandem mass spectrometry, computational methods, and NMR. Journal of Mass Spectrometry, 2008. 43(12): p. 1581-1617.

70. Mihaleva, V. V., et al., MetIDB: A Publicly Accessible Database of Predicted and Experimental 1H NMR Spectra of Flavonoids. Analytical Chemistry, 2013. 85(18): p. 8700-8707.

71. Beekmann, K., et al., Flavonoid conjugate biosynthesis and identification using the metabolites identification database (MetIDB). In preparation.

72. Day, A. J., et al., Human metabolism of dietary flavonoids: Identification of plasma metabolites of quercetin. Free Radical Research, 2001. 35(6): p. 941-952.

73. Mullen, W., C. A. Edwards, and A. Crozier, Absorption, excretion and metabolite profiling of methyl-, glucuronyl-, glucosyl-and sulpho-conjugates of quercetin in human plasma and urine after ingestion of onions. British Journal of Nutrition, 2006. 96: p. 107-116.

74. Hosoda, K., et al., Identification and quantification of daidzein-7-glucuronide-4'-sulfate, genistein-7-glucuronide-4'-sulfate and genistein-4',7-diglucuronide as major metabolites in human plasma after administration of kinako. Analytical and Bioanalytical Chemistry, 2010. 397(4): p. 1563-1572.

75. Hosoda, K., T. Furuta, and K. Ishii, Metabolism and disposition of isoflavone conjugated metabolites in humans after ingestion of kinako. Drug Metabolism and Disposition, 2011. 39(9): p. 1762-1767. 
76. Yuan, B., et al., Absorption and plasma disposition of genistin differ from those of genistein in healthy women. Journal of Agricultural and Food Chemistry, 2012. 60(6): p. 1428-1436.

77. Needs, P. W. and P. A. Kroon, Convenient syntheses of metabolically important quercetin glucuronides and sulfates. Tetrahedron, 2006. 62(29): p. 6862-6868.

78. Bouktaib, M., A. Atmani, and C. Rolando, Regio- and stereoselective synthesis of the major metabolite of quercetin, quercetin-3-O- $\beta$-d-glucuronide. Tetrahedron Letters, 2002. 43(35): p. 6263-6266.

79. Marshall, T., P. Shult, and W. W. Busse, Release of lysosomal enzyme beta-glucuronidase from isolated human eosinophils. Journal of Allergy and Clinical Immunology. 82(4): p. 550-555.

80. Shimoi, K., et al., Deglucuronidation of a favonoid, luteolin monoglucuronide, during inflammation. Drug Metabolism and Disposition, 2001. 29(12): p. 1521.

81. Shimoi, K. and T. Nakayama, Glucuronidase deconjugation in inflammation, in Methods in Enzymology. 2005, Academic Press. p. 263-272.

82. Bosslet, K., et al., Elucidation of the mechanism enabling tumor selective prodrug monotherapy. Cancer Research, 1998. 58(6): p. 1195.

83. Sperker, B., et al., Expression and function of $\beta$-glucuronidase in pancreatic cancer: potential role in drug targeting. Naunyn-Schmiedeberg's Archives of Pharmacology, 2000. 362(2): p. 110-115.

84. Roura, E., et al., The effects of milk as a food matrix for polyphenols on the excretion profile of cocoa ( - )-epicatechin metabolites in healthy human subjects. British Journal of Nutrition, 2008. 100(4): p. 846-851.

85. Rodriguez-Mateos, A., et al., Influence of sugar type on the bioavailability of cocoa flavanols. British Journal of Nutrition, 2012. 108(12): p. 2243-2250.

86. Meng, X., et al., Urinary and plasma levels of resveratrol and quercetin in humans, mice, and rats after ingestion of pure compounds and grape Juice. Journal of Agricultural and Food Chemistry, 2004. 52(4): p. 935-942.

87. Guo, Y., et al., Dietary fat increases quercetin bioavailability in overweight adults. Molecular Nutrition \& Food Research, 2013. 57(5): p. 896-905.

88. Bohn, T., Dietary factors affecting polyphenol bioavailability. Nutrition Reviews, 2014. 72(7): p. 429.

89. Brand, W., et al., The effect of co-administered flavonoids on the metabolism of hesperetin and the disposition of its metabolites in Caco-2 cell monolayers. Molecular Nutrition \& Food Research, 2010. 54(6): p. 851-860.

90. Aura, A.-M., Microbial metabolism of dietary phenolic compounds in the colon. Phytochemistry Reviews, 2008. 7(3): p. 407-429.

91. Morito, K., et al., Interaction of phytoestrogens with estrogen receptors alpha and beta. Biological and Pharmaceutical Bulletin, 2001. 24(4): p. 351-356.

92. Hwang, C. S., et al., Isoflavone metabolites and their in vitro dual functions: They can act as an estrogenic agonist or antagonist depending on the estrogen concentration. The Journal of Steroid Biochemistry and Molecular Biology, 2006. 101(4-5): p. 246-253.

93. Kay, C. D., Rethinking paradigms for studying mechanisms of action of plant bioactives. Nutrition Bulletin, 2015. 40: p. 335-339.

94. Osborne, C. K., K. Hobbs, and J. M. Trent, Biological differences among MCF-7 human breast cancer cell lines from different laboratories. Breast Cancer Research and Treatment, 1987. 9: p. 111-121.

95. Strikwold, M., et al., Combining in vitro embryotoxicity data with physiologically based kinetic $(P B K)$ modelling to define in vivo dose-response curves for developmental toxicity of phenol in rat and human. Archives of Toxicology, 2013. 87(9): p. 1709-1723. 
96. Louisse, J., et al., Prediction of in vivo developmental toxicity of all-trans-retinoic acid based on in vitro toxicity data and in silico physiologically based kinetic modeling. Archives of Toxicology, 2015. 89(7): p. 1135-1148.

97. Polak, S., In vitro to human in vivo translation - pharmacokinetics and pharmacodynamics of quinidine. ALTEX, 2013. 30(3): p. 309-318.

98. Gülden, M. and H. Seibert, In vitro-in vivo extrapolation: estimation of human serum concentrations of chemicals equivalent to cytotoxic concentrations in vitro. Toxicology, 2003. 189(3): p. 211-222.

99. Simcyp. Simcyp prediction tools -fu. [Online]. Available from: https://members.simcyp.com/account/tools/fu/). [Accessed: 15th July 2015]. 2014 [cited 2014.

100. Boersma, M. G., et al., Regioselectivity of phase II metabolism of luteolin and quercetin by UDP-glucuronosyl transferases. Chemical Research in Toxicology, 2002. 15(5): p. 662-670.

101. EFSA, Guidance on human health risk-benefit assessment of foods. EFSA Journal, 2010. 8(7): p. 1673.

102. EFSA, Opinion of the scientific committee on a request from EFSA related to a harmonised approach for risk Assessment of substances which are both genotoxic and carcinogenic. EFSA Journal, 2005. 3(10): p. 282. 
Chapter 6 
Summary

Acknowledgements

About the author

List of publications

Overview of training activities 


\section{Summary}

Despite numerous reports on possible mechanisms of action of flavonoids for beneficial health effects derived from in vitro bioassays, in vivo biological effects of flavonoids are contradictory and inconclusive. This is because most in vitro studies have been performed with flavonoid aglycones at high concentrations ignoring extensive metabolism of flavonoids in vivo. The aim of the present $\mathrm{PhD}$ thesis was to gain quantitative insight in dosedependent plasma concentrations of flavonoid aglycones and their metabolites using physiologically based kinetic (PBK) modeling, and the flavonoids quercetin, genistein and hesperetin as model compounds. To this end, PBK models were developed by integrating in vitro kinetic parameters for metabolic conversions of flavonoids to different conjugates and by fitting excretion parameters to literature available in vivo data. The PBK models obtained were validated against available in vivo data on metabolic plasma and urinary profiles of flavonoids of interest. Altogether, the results presented in this thesis show that PBK modeling is a powerful tool, not only to gain insight in the dose-dependent plasma concentrations of flavonoid aglycones and their metabolites, but also to tentatively identify the type and conjugation position of the circulating metabolites. These outcomes can be used to guide experimental design of in vitro studies by enabling definition of the major available forms of a flavonoid in the systemic circulation, providing information on the type and conjugation position and thus the type of conjugates to be preferably tested in vitro. The present thesis also illustrated that PBK modeling can be used to identify inter- and intraspecies differences in the absorption, distribution, metabolism, and excretion of flavonoids taking quercetin as the model compound. Moreover, it was demonstrated that the PBK models can facilitate the translation of in vitro concentration-response curves for biological effects to in vivo doseresponse curves, allowing to determine at which dose levels the in vitro biological effects can be expected in humans in vivo. Overall, PBK modeling thus provides a useful additional research tool for studies on pharmacokinetics and pharmacodynamics of flavonoids presenting opportunities that are not easily provided by other methods. To implement the PBK model based approach developed in the present thesis for a wider range of flavonoids, future studies should focus on further refinement of the PBK models, development of adequate in vitro biological models of flavonoid mediated effects, and use of PBK model based reverse dosimetry to advance our understanding on in vivo effects of flavonoids. 


\section{Acknowledgments}

Frist of all, I would like to express my sincere gratitude toward my promoter, Ivonne Rietjens. Ivonne thanks a lot for your support, guidance, patience, motivation, and immense knowledge. You always give me critical comments on my work, which make me realize that I still have a lot to learn to be as critical as you are. In these past years, I am still amazed on how efficient you can be. I really appreciate your quick responses especially at the final phase of my $\mathrm{PhD}$ study.

My sincere thank also goes to my co-supervisor Ans Punt. Ans thank you very for continuous support. You have taught me a lot about PBK modeling. I feel fortunate to have you as my daily supervisor. Our working styles very match with each other, at least this is what I think. I feel comfortable to work with you. You were always available and willing to help me whenever I faced problems. Even when you were on maternity leave or changing your job, I still felt like you were always there to help me.

I would like to thank my colleagues from Toxicology. Let's start with my buddy Bert Spenkelink, Bert thanks a lot for your dedicated work, you have been working very hard to help me with my experiments and technical problems. You taught me a lot about UPLC/HPLC until the point that I would like to be a great technician like you. I really enjoy listening to your stories, especially the story about your parents during the second world war. This story makes me realize how lucky I am to be born during peaceful times. Big brother, Nacho (Ignacio), thanks a lot for everything that you have helped me with. I am always grateful for your support, advice, and enthusiasm. I feel super comfortable around you until the point that I can say whatever I think. Big brother just try to stay positive and be optimistic. Rozaini, a little flower from Malaysia, thanks a lot for your help. I think I started to talk more when I moved to your office. I am always grateful for your friendship and encouragement. You always packed foods for me and let me stay in your room when I have nowhere to go. I enjoyed travelling with you as both of us like to take a photo, even though you are more a model than a photographer. Aziza, a flower from Sudan, thanks a lot for your support and friendship (do not cry when you read this message). It still amazes me how fast you can talk. You are a good cook, I enjoyed your food a lot especially the paprika rice. Lenny, thanks a lot for your help and friendship. You are a good little sister. I enjoyed talking with you. Laura thanks a lot for an introduction about cell cultures. Karsten thanks for helping me with the PamGene experiments. I would like to express my gratitude to Irene, Gré, and Lidy for helping me out with administrative work. Thank you Sebas, Hans, Hans B, Marélle, Jochem, Nico, Ans S, Jac and Tinka for technical input and grate talks. And of course, I also would 
like to thank all other (former) colleagues for a grate friendship, nice working atmosphere, and many fun activities: Abdul, Agata, Alexandros, Amer, Arif, Artem, Ashraf, Barae, Diego, Elise, Erryana, Georgia, Hequn, Jia, Justine, Kornphimol (P’Tom), Letty, Linda, Lu, Marcia, Marije, Marta, Mebrahtom, Mengying, Myrto, Nynke, Reiko, Samantha, Sophie, Suparmi, Sunday, Suzanne, and Wasma. I also would like to thank my former students for their contribution to this thesis: Nooshin, Hanna, Yvonne, Ming, Eva, Leli, Karel, and Wouter.

I am always grateful to the Thai community in Wageningen for all the times that they made me feel at home. I enjoyed very much the Thai dinner and music. I still remember the time that I had to teach you guys how to make "curry puff". It was quite a challenge for me.

I also would like to thank my friends in Thailand for their support and encouragement throughout my PhD study. Nong and Nui, thank you very much for your friendships and for listening to my problems.

Last and most importantly, I would like to thank my parents for their love, support and encouragement. I know there are very pound of me. They always wish for me to have a good education.

Everyone thank you again, even though I am not good at expressing myself. I would like all of you to know how grateful I am, without the support from all of you I could not have completed my PhD study. Wherever I am, I will always remember all of you.

Rungnapa Boonpawa 


\section{About the author}

Rungnapa Boonpawa was born on $20^{\text {th }}$ January 1985 in Kalasin province, Thailand. She grew up there until she finished her secondary education at Rongkham School. From 2004-2009, she received a One District One Scholarship (ODOS) from Thai government to complete her BSc degree in food technology at Van Hall Larenstein University of Applied Science; part of

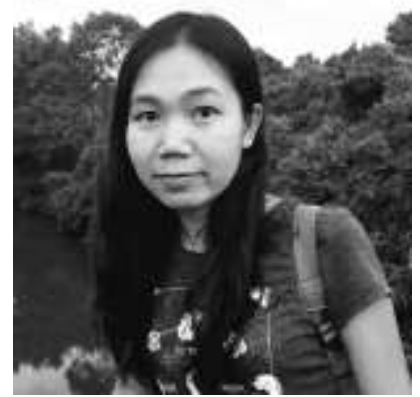
Wageningen University \& Research in The Netherlands. From 2009-2016, Rungnapa received a Royal Thai Government Scholarship offered by Ministry of Science and Technology of Thailand to pursuit her study in MSc and PhD degree. In 2009, Rungnapa started her MSc study in food safety at Wageningen University \& Research, where she performed her thesis at Division of Toxicology on a topic of effect of flavonoids on reducing bioactivation and carcinogenic risk of foodborne genotoxic and carcicogenic safrole. She subsequently finished her internship at RIKILT Institute of Food Safety (Wageningen, The Netherlands) on the topic of development of SPR-based biosensor for detecting organic engineered nanoparticles in food. From February 2012, she started her PhD project presented in this thesis at Division of Toxicology, Wageningen University \& Research. Apart from the project, she was a web editor for Division of Toxicology website. During the four years, she followed numbers of postgraduate courses in toxicology required for registration as a European Toxicologist. After her $\mathrm{PhD}$ study, she will go back to Thailand to start her academic career at Faculty of Natural Resources and Agro-Industry, Kasetsart University (Sakon Nakhon province, Thailand). 


\section{List of publications}

Martati E, Boonpawa R, van den Berg JHJ, Paini A, Spenkelink A, Punt A, Vervoort J, van

Bladeren PJ, \& Rietjens IMCM (2014). Malabaricone C-containing mace extract inhibits safrole bioactivation and DNA adduct formation both in vitro and in vivo. Food Chem Toxicol 66: 373-384.

Boonpawa R, Moradi N, Spenkelink A, Rietjens IMCM, \& Punt A (2015). Use of physiologically based kinetic (PBK) modeling to study interindividual human variation and species differences in plasma concentrations of quercetin and its metabolites. Biochem Pharmacol 98: 690-702.

Boonpawa R, Spenkelink A, Rietjens IMCM, \& Punt A (2014). A physiologically based kinetic (PBK) model describing plasma concentrations of quercetin and its metabolites in rats. Biochem Pharmacol 89: 287-299.

Boonpawa R, Spenkelink A, Punt A, \& Rietjens IMCM. In vitro-in silico based analysis of the dose-dependent in vivo estrogenicity of the soy phytoestrogen genistein in humans. Submitted.

Boonpawa R, Spenkelink A, Punt A, \& Rietjens IMCM. Physiologically based kinetic modeling of hesperidin metabolism and its use to predict in vivo effective doses in humans. Submitted. 


\section{Overview of completed training activities}

\section{Discipline specific courses}

General Toxicology, Wageningen University \& Research (WUR), 2012

Molecular Toxicology, Postdoctoral Education in Toxicology (PET), 2013

Cell Toxicology, PET, 2013

Organ Toxicology, PET, 2013

Mutagenesis and Carcinogenesis, PET, 2013

Immunotoxicology, PET, 2014

Epidemiology, PET, 2014

Pathobiology, PET, 2015

Medical and Forensic Toxicology, PET, 2015

\section{Meetings}

$18^{\text {th }}$ international congress on In Vitro Toxicology ESTIV2014, Egmond aan Zee, The

Netherlands, poster, 2014

Dutch Society of Toxicology (NVT) annual meeting, Zeist, The Netherlands, poster, 2015

\section{General courses}

Teaching and Supervising Thesis students, WUR, 2012

VLAG PhD week, VLAG, 2012

Laboratory Animal Science, PET, 2014

Scientific Writing, Wageningen Graduate Schools, 2015

Risk Assessment, PET, 2015

Lecturing, WUR, 2015

\section{Optional}

Preparing $\mathrm{PhD}$ research proposal

Attending Scientific Presentation, Division of Toxicology, WUR, 2012-2016

Environmental Toxicology (TOX-30806), WUR (MSc course), 2012

$\mathrm{PhD}$ excursion to UK, Division of Toxicology, WUR, 2013

\section{Approved by Graduate School VLAG}


The studies described in this thesis were financially supported by the Ministry of Science and Technology of Thailand through a Royal Thai Government Scholarship, Thailand.

Financial support from Wageningen University \& Research for printing this thesis is gratefully acknowledged.

Cover \& Layout design: Rungnapa Boonpawa

Printed by: Digiforce, Vianen, www.proefschriftmaken.nl 